\title{
Bacterial Electron Transfer Chains Primed by Proteomics
}

\section{Hans J.C.T. Wessels ${ }^{1}$, Naomi M. de Almeida ${ }^{2}$, Boran Kartal ${ }^{2,3}$ and Jan T. Keltjens ${ }^{2, *}$}

${ }^{1}$ Nijmegen Center for Mitochondrial Disorders, Radboud Proteomics Centre, Translational Metabolic Laboratory, Department of Laboratory Medicine, Radboudumc,

Geert Grooteplein zuid 10, 6525 GA, Nijmegen, The Netherlands

${ }^{2}$ Department of Microbiology, Institute of Water and Wetland Research, Radboud University Nijmegen, 6525 AJ, Nijmegen, The Netherlands

${ }^{3}$ Department of Biochemistry and Microbiology, Laboratory of Microbiology, Gent University, K.L. Ledeganckstraat 35, 9000 Gent, Belgium

*Corresponding author: Department of Microbiology, IWWR, Faculty of Science, Radboud University of Nijmegen, Heyendaalseweg 135, 6525 AJ Nijmegen, The Netherlands. Tel: +31.24.3653437. Fax: +31.24.3652830. E-mail: J.Keltjens@science.ru.nl.

Running title: respiration primed by proteomics

Key words: Respiratory complexes- Proteomics - Differential expression Methanothermobacter thermautotrophicus - anammox bacteria - Mitochondria 


\begin{abstract}
Electron transport phosphorylation is the central mechanism for most prokaryotic species to harvest energy released in the respiration of their substrates as ATP. Microorganisms have evolved incredible variations on this principle, most of these we perhaps do not know, considering that only a fraction of the microbial richness is known. Besides these variations, microbial species may show substantial versatility in using respiratory systems. In connection herewith, regulatory mechanisms control the expression of these respiratory enzyme systems and their assembly at the translational and post-translational levels, to optimally accommodate changes in the supply of their energy substrates. Here, we present an overview of methods and techniques from the field of proteomics to explore bacterial electron transfer chains and their regulation at levels ranging from the whole organism down to the Ångstrom scales of protein structures. From the survey of the literature on this subject, it is concluded that proteomics, indeed, has substantially contributed to our comprehending of bacterial respiratory mechanisms, often in elegant combinations with genetic and biochemical approaches. However, we also note that advanced proteomics offers a wealth of opportunities, which have not been exploited at all, or at best under-exploited in hypothesis-driving and hypothesisdriven research on bacterial bioenergetics. Examples obtained from the related area of mitochondrial oxidative phosphorylation research, where the application of advanced proteomics is more common, may illustrate these opportunities.
\end{abstract}




\section{Table of contents}

Abbreviations

1. Introduction

2. Methodology of Proteomics

2.1. Introduction to mass spectrometry

2.1.1. The mass spectrum

2.1.2. The ionisation process

2.1.3. Mass analysers

2.1.4. Peptide sequencing: Tandem mass spectrometry

2.1.5. Analysis of complex samples: LC-MS/MS

2.2. Protein identification

2.2.1. Peptide centric (bottom-up) proteomics

2.2.2. Protein centric (top-down) proteomics

2.3. Protein quantitation by mass spectrometry

2.3.1 Protein quantitation: Principles

2.3.2 Label-free and stable isotope labelling quantitation methods

2.3.3 Untargeted and targeted approaches

3. Bacterial electron transfer chains and their regulation: global approaches

3.1. 2DE-based methods and shotgun proteomics

3.1.1. Methods based on 2D IEF SDS-PAGE, the classical approach

3.1.2. Shotgun proteomics

3.2. Membrane-bound systems

3.2.1. Analysis of membrane-bound proteins

3.2.2. Membrane-bound protein complexes

3.3. Differential expression 
4. Respiratory protein complexes in assembly

4.1. Structural aspects of respiratory complexes

4.1.1. Protein purification

4.1.2. Primary structure information

4.1.3 On track to secondary, tertiary and quaternary structures of respiratory complexes

4.1.4. Protein-lipid interactions

4.1.5. Membrane protein complexes studied in thin air

4.1.6. Putting structural information in a timed perspective

4.2. Post-translational modifications

4.2.1 Protein phosphorylation

4.2.2 Protein acetylation

4.2.3 Protein methylation

4.2.4 Oxidative stress-related post-translational modifications

5. Assemblages of respiratory protein complexes

$5.1 \quad$ Supercomplexes

5.1.1 Mitochondrial supercomplexes

5.1.2 Bacterial supercomplexes

5.2 The interactome

5.2.1 Assembly of bacterial respiratory complexes

5.2.2 The far from complete picture of interaction partners and interaction dynamics

6. Perspectives 
ABBREVIATIONS

2DE

16-BAC

AP-MS, q-AP-MS

AQUA

BNE

CBB

CE

CID

CNE

CTAB

CX-MS

Da

DDA

DDM

DIA

DIGE

EI

emPAI

ESI

ETC

ETD

ETP

ETS

FAB two-dimensional gel electrophoresis

16-benzyldimethyl-n-hexadecylammonium chloride

(quantitative) affinity purification

absolute quantitation

blue native gel electrophoresis

Coomassie Brilliant blue

capillary electrophoresis

collision-induced dissociation

clear (or colourless) native gel electrophoresis

cetyltrimethyl-ammonium bromide

chemical crosslinking coupled with MS

dalton

data-dependent analysis

dodecyl- $\beta$-D-maltoside

data-independent analysis

difference gel electrophoresis

electron ionisation

exponentially modified protein abundance index

electrospray ionisation

electron transfer chain

electron-transfer dissociation

electron transport phosphorylation

electron transfer system

fast atom bombardment 
FT-ICR

FWHM

GPI

GRAVY

HDX-MS

ICP-MS

ICAT

IEF

IM-MS

IMAC

IPG

IT

iTRAQ

LC

LC-MS/MS

LILBID

LIT

LMW

m

$m / z$

MALDI-TOF

MIRAGE

MRM

MS

$\mathrm{MS}^{\mathrm{n}}$
Fourier transform ion-cyclotron-resonance

full width at half maximum

glycosylphosphaditylinisositol

grand average of hydropathicity

hydrogen/deuterium exchange coupled with MS

inductively coupled plasma mass spectrometry

isotope coded affinity tags

isoelectric focusing

ion-mobility MS

immobilised metal affinity chromatography

immobilised $\mathrm{pH}$ gradient

ion trap

isobaric tags for relative and absolute quantitation

liquid chromatography

liquid chromatography online tandem mass spectrometry

laser-induced liquid bead ion-desorption

linear ion trap

low-molecular weight

relative mass

mass to charge ratio

matrix assisted laser desorption ionisation time-of-flight

metal isotope native radio autography in gel electrophoresis

multiple reaction monitoring

mass spectrometry

higher order mass spectrometry experiment 
MudPIT

$\mathrm{NDH}$

ORF

OXPHOS

PAGE

$\mathrm{p} I$

PIC

Pmf

PTM

Q

q

QqQ

Q-TOF

RCS, RES, RNS, ROS

RMP

RP

SDH

SDS

Smf

SC

SCX

SILAC

SRM

TAP-MS multidimensional protein identification technology

NADH:quinone oxidoreductase (complex I)

open-reading frame

oxidative phosphorylation, more specifically in mitochondria

polyacrylamide gel electrophoresis

isoelectric point

phenyl isothiocyanate labelling

proton-motive force

post-translational modifications

analytical quadrupole

non-analytical quadrupole

triple quadrupole

quadrupole time of flight

reactive chlorine, electrophilic, nitrogen, oxygen species

(bacterial) respiratory membrane-bound protein complex

reversed-phase

succinate dehydrogenase (complex II)

sodium dodecyl sulphate

sodium-motive force

supercomplex

strong cation exchange chromatography

stable-isotope labelling with amino acids in cell culture

selected reaction monitoring

tandem affinity purification 
Th

$\mathrm{TMH}$

TMT

TOF

$\mathrm{u}$

Z thomson

transmembrane helix

tandem mass tags

time of flight

atomic mass unit

charge number 


\section{INTRODUCTION}

Respiration is a hallmark of life. By this principle, also known as Electron Transport Phosphorylation (ETP) or Oxidative Phosphorylation (OXPHOS), electrons derived from the oxidation of organic or inorganic substrates are transferred to a terminal electron acceptor (AlAttar and de Vries, 2013). Substrate oxidation and reduction, and intermediary electron transfer is catalysed by respiratory membrane-bound protein complexes (RMPs). Lowmolecular-weight compounds dissolved either in the membrane, such as quinones, or in the cytoplasmic (NADH, ferredoxins) and periplasmic (c-type cytochromes, blue copper proteins) solutes assist as electron shuttles between the RMPs. These systems are organised such that the energy released in these redox processes is conserved as a proton-motive force ( $\mathrm{pmf}$ ) or a sodium-motive force (smf) across the cell membrane. This force drives the synthesis of ATP by the membrane-bound, $\mathrm{H}^{+}$(or $\mathrm{Na}^{+}$)-translocating ATP synthase. The textbook example of an Electron Transfer System (ETS) is the mitochondrion of eukaryotes. In mitochondria, electrons from NADH or succinate oxidation enter the chain via NADH:quinone oxidoreductase (NDH, complex I) or succinate dehydrogenase (SDH, complex II), respectively, pass via a quinol:cytochrome $c$ oxidoreductase (complex III) to end in the terminal oxidase (complex IV) that reduces $\mathrm{O}_{2}$ to $\mathrm{H}_{2} \mathrm{O}$. Energy is conserved by the ATP synthase (complex V).

Eukaryotes generally respire only oxygen by using electrons generated from the oxidation of organic substrates (sugars, fatty acids, amino acids). Aerobic microorganisms use oxygen as well, but they have a diversity of oxidases at their disposal that can cope with an enormous range of environmental oxygen concentrations (Hemp and Gennis, 2008; Borisov et al., 2011; Han et al., 2011). Prokaryotes also can use many other (in)organic electron acceptors in anaerobic respiration in species-dependent ways (Croal et al., 2004; Matias et al., 2005; Zumft and Kroneck, 2007; Heimann et al., 2007; Simon and Kern, 2008; Kraft et al., 2011; 
Richter et al., 2012; Shan et al., 2012; Grein et al., 2013; Tosha and Shiro, 2013; Suh et al., 2014), whereas an almost unlimited number of (in)organic substrates serve as electron donors, provided energy is gained from the redox processes. The conversion of each specific set of substrates requires its dedicated RMPs that are organised as linear or branched electron transfer chains. Besides this versatility, prokaryotes may display a tremendous respiratory flexibility to cope with changes in the supply of energy substrates. Many different ETSs from model organisms have been studied by now in varying levels of detail (see above references). Still, we most likely have only a limited notion of these systems: only a minor fraction of the microbial species richness is known. However, our insight into this richness increases at an incredible pace, supported by the power of (meta)genomics and (meta)transcriptomics (Godzik, 2011; Caro-Quintero and Konstantinidis, 2012; Temperton and Giovannoni, 2012; Oren and Garrity, 2014; Gerber, 2014; Brown et al., 2015; Faust et al., 2015; Land et al., 2015; Méndez-Garcíaet et al., 2015; Ponomarova et al., 2015; Suenaga, 2015; van Schaik, 2015). From metagenome assemblies it can be inferred that microorganisms have evolved overwhelming variations of already known RMPs, permitting a species to occupy a specific niche. Many of the genome assemblies deal with species of completely new phyla, lacking cultured representatives with known physiology and metabolism. These new species may metabolise their substrates by new pathways whose nature is hidden in DNA sequences as genes coding for "hypothetical" or "conserved" proteins, or genes that have been annotated erroneously. Moreover, even if a certain gene product is highly homologous to a protein of known function, this does not imply that the gene product is functional or has the predicted function. Functional expression may be highly complicated requiring the association of other partners that are not immediately identified by DNA analyses, no matter how advanced. This is particularly the case for RMPs. 
RMPs are usually composed of different subunits in fixed ratios. In prokaryotes, for instance, NDH consists of 13 subunits, while the same complex (I) in eukaryotic mitochondria comprises about 45 subunits (Mimaki et al., 2012). After translation, each of the subunits undergoes modifications, resulting in mature functional complexes (Section 5.2.1). Next, post-translational modifications (PTMs) may involve the addition of specific groups (phosphorylation, acetylation, methylation, etc.) to specific amino acids for structural integrity or to control enzyme activity (Section 4.2). All in all, the assembly and PTMs must be welltimed and carefully controlled events, which are regulated in the way that the living cell can immediately and properly respond to environmental changes.

By now, it has become clear that the above-depicted view of ETCs as being composed of individual RMPs linked by mobile electron carriers is more complicated. RMPs appear to combine into supercomplexes (Section 5.1). The modes of supercomplex (SC) formation and factors directing their formation has become one of the most exciting recent advances in the research on electron transfer systems.

All of the phenomena outlined above boil down to the fact that proteins are composed of specific amino acid sequences that are subjected to cleavage, insertion of (non-peptide) cofactors, the addition of functional groups, small molecules or other chemical modifications: In the end, it is all about chemistry. This chemical a priori makes their study amenable to one of the most powerful, sensitive and accurate methods in the field, mass spectrometry (MS). In the last two decades, we have seen incredible developments in MS instrumentation and methodologies, as well as in the generation of massive amounts of high-quality data and computer software-assisted handling of these data. Hereby, MS-supported protein research has become a field on its own: proteomics. In this chapter, we will present an overview of the way proteomics has added to the fundamental understanding of electron transport systems and their regulation, and particularly in the way applications of the proteomics toolbox may 
contribute to the future understanding of bacterial respiration. We will do so from the global and enzyme-targeted perspectives. The global approach aims at mapping the complete protein complement and all its modifications ("proteoforms"; Smith et al., 2013) of an organism, and

how this protein complement is modified in response to environmental changes. This approach is mainly discovery-based and can be used to generate new hypotheses. In contrast, the enzyme-targeted, hypothesis-driven research is directed towards the insight in the structure and functioning of an enzyme or enzyme system as isolated, taking advantage of the methodology offered by proteomics. Proteomics supports both approaches by bottom-up and top-down methods. In the bottom-up direction, which we will also refer to as peptide-centric, proteins in a sample are first proteolytically or chemically cleaved. Hereafter, peptide fragments are analysed by MS as to identify (and quantify) the proteins present in the sample or to get structural information in case of an enzyme-targeted research question. The topdown, protein-centred approach takes the whole protein (complex) as the starting point. Both in bottom-up and top-down directions, proteomics offers an arsenal of MS analytical methods. To make a non-expert reader familiar with these methods, we will start our review with an introduction to proteomics.

\section{METHODOLOGY OF PROTEOMICS}

Unambiguous protein identification, characterisation and quantitation by mass spectrometry are invaluable to gain molecular insight into the complicated protein complexes of the electron transport system. The possibility to fully dissect protein complexes at the molecular level using only small sample amounts with high-throughput is unique to MS and often crucial in protein research. Mass spectrometry is used to accurately determine a (poly)peptide's mass, amino acid sequence (or identity), quantity, post-translational modifications, and interaction partners. Even more elaborate experiments can be performed to 
unravel fine structural details such as disulphide bridge arrangements, cross section and protein-protein interaction sites. Continuous advancements in hardware, software, and method development rapidly expand the capabilities of MS in protein research, but also make it difficult for non-experts to grasp the possibilities and limitations of this technique. In this section we will provide some basics of mass spectrometry and proteomics methodology that are relevant for the analysis of ETS protein complexes (see for a more detailed discussion: de Hoffmann and Stroobant, 2007).

\subsection{Introduction to mass spectrometry}

To understand how proteomics methodology works, it is important to have a minimal understanding of mass spectrometry hardware and how experiments are conducted. We will first explain what information can be obtained from a mass spectrum and introduce the units that are used in this review.

\subsubsection{The mass spectrum}

In contrast to its name, a mass spectrometer does not measure the mass of a (poly)peptide directly per se. Rather, it measures the mass to charge ratio $(\mathrm{m} / \mathrm{z})$ in thomson $(\mathrm{Th})$ of ions from which the relative mass $(m)$ can be derived if the charge number of the ion $(z)$ is known. The relative mass $(m)$ is a dimensionless number which is defined as the mass of an atom, a molecule or an ion divided by one-twelfth of the mass of the ${ }^{12} \mathrm{C}$ carbon atom. The charge number $(z)$ is the total charge on an ion divided by the elementary charge $(e), 1.602177 \times 10^{-19}$ C. The thomson is defined as $1 \mathrm{Th}=1 \mathrm{u} / e=1.036426 \times 10^{-8} \mathrm{~kg}^{-\mathrm{C}^{-1}}$. The mass of a (poly)peptide in proteomics is described by its monoisotopic mass, average mass, or nominal mass in atomic mass units ( $\mathrm{u}$ or amu), which is called the dalton (Da). The monoisotopic mass of an ion is calculated using the "exact" mass of the predominant isotope of each element. The 
average mass (or chemical mass) is a weighted average of the natural isotopes for the atomic mass of each element. The nominal mass is the mass of the predominant isotope of each element rounded to the nearest integer value. At first sight, these mass definitions seem to differ only marginally. However, for large biopolymers such as (poly)peptides the differences become significant, especially when taking the mass accuracy of modern mass spectrometers into account. For example, if we consider a carbamidomethylated (CAM) tryptic peptide from bovine serum albumin (BSA), YIC(cam)DNQDTISSK (chemical formula: $\mathrm{C}_{59} \mathrm{H}_{94} \mathrm{~N}_{16} \mathrm{O}_{24} \mathrm{~S}_{1}$ ), significant mass differences are observed for the different mass calculations: monoisotopic mass, 1442.6348 u; average mass, 1443.5376 u; nominal mass, 1442.0000 u. The monoisotopic mass is typically used in proteomics experiments to describe the mass of a (poly)peptide if its isotopic peaks are resolved in the mass spectrum. A mass spectrum plots the intensity of an ion versus its mass to charge ratio. Figure $1<$ Figure $\mathbf{1}>$ shows the isotopic peaks in an electrospray ionisation mass spectrum for ions of the tryptic BSA peptide

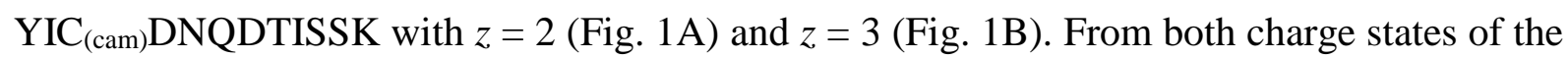
ion, we derive the same monoisotopic mass: Mr 1442.6358 Da from $m / z 722.3257[\mathrm{M}+2 \mathrm{H}]^{2+}$ and $\mathrm{Mr}$ 1442.6381 Da from $\mathrm{m} / \mathrm{z} 481.8872[\mathrm{M}+3 \mathrm{H}]^{3+}$. The charge state of an ion can be determined from the mass spectrum on the basis of the observed distance in Th between the different isotopic peaks. Since the isotopic peaks differ by $1 \mathrm{Da}$, the observed distance will be $1 / z$. Therefore, the charge state of an ion can be calculated as $z=1 /$ [observed distance in Th]. For the ion in Figure 1A, we observe a mass difference of 0.5 Th between the isotopic peaks, which determines the charge number of the ion: $z=1 / 0.5=2$. Similarly, for the ion in Figure 1B, the observed mass difference of 0.33 Th establishes its charge number: $z=1 / 0.33$ = 3. Computer algorithms can also generate a "deconvoluted" spectrum in which the spectral information from each charge state peak of a single compound (identical $m$ but different $z$ ) is combined into a single spectral peak with mass $[\mathrm{M}]$ or $[\mathrm{M}+\mathrm{H}]^{1+}$. This is particularly useful for 
large biomolecules such as intact proteins, which are usually detected with a wide range of different charges in a single electrospray ionisation mass spectrum. Figure $2 \mathrm{~A}<$ Figure $2>$ shows the electrospray ionisation mass spectrum (as recorded) of bovine cytochrome $c$, which was detected with charge states in the range of $z=7$ up to $z=21$. The deconvoluted spectrum shown in Figure 2B is much easier to interpret as it shows only a single spectral peak for cytochrome $c$ with a neutral mass of $\mathrm{Mr} 12351.3059$ Da (calculated neutral mass for $\mathrm{C}_{560} \mathrm{H}_{873} \mathrm{~N}_{148} \mathrm{O}_{156} \mathrm{~S}_{4} \mathrm{Fe}_{1}$ is $\mathrm{Mr} 12351.3161 \mathrm{Da}$; measured mass error: 0.0102 Da / 0.8 ppm). The isotopic distribution observed in mass spectra contains even more information than the mere charge number of an ion. The natural occurrence of isotopes differs between elements. Therefore, the relative intensities from the different isotopic peaks of a molecule reflect directly its elemental composition. Thus far, besides accurate mass, the isotopic pattern is mostly used to verify the identity of small molecules by mass spectrometry. To summarise, a mass spectrum contains the mass to charge ratio $(\mathrm{m} / \mathrm{z})$ of an ion from which $m$ and $z$ can be derived, information about the elemental composition of the ion from its isotopic pattern, and the detected intensity of the ion in arbitrary units.

\subsubsection{The ionisation process}

Any mass spectrometry experiment starts with the ionisation process, which takes place in the ion source. Ionisation of (poly)peptides is essential in mass spectrometry since electrical and/or magnetic fields are applied by the mass analyser to subsequently transport, manipulate, and detect molecules with a net positive or negative charge (ions). During ionisation, a (poly)peptide acquires a net positive or negative charge by gaining or losing protons. Besides charging, the ionisation process must ensure that ions are available in the gas phase for the mass analyser. This may seem trivial, but efficient ionisation of non-volatile biomolecules such as (poly)peptides has been a challenge that was solved by the introduction of soft 
ionisation techniques of “electrospray ionisation” (ESI) and "matrix assisted laser desorption ionisation” (MALDI) in 1984 (Yamashita and Fenn, 1984) and 1985 (Karas et al., 1985), respectively. Till then, harsh ionisation techniques such as "electron ionisation” (EI) (de Hoffmann and Stroobant, 2007) and the softer "fast atom bombardment” (FAB) (Barber et al., 1981) methods were used to ionise (poly)peptides but these techniques were often inefficient or induced undesired fragmentation of the analyte prior to detection. Both ESI and MALDI are non-destructive ionisation methods for (poly)peptides that achieve high ionisation efficiency to enable routine mass spectrometric analysis of (poly)peptides.

In MALDI, (poly)peptides are co-crystalized with a small organic compound on the surface of a sample plate. Following crystallisation, the sample plate is inserted via a vacuum lock into the mass spectrometer where it is placed onto a XYZ stage and transported into the MALDI source chamber. On the XYZ stage the target is aligned with a laser beam and ion optics of the mass analyser. Once positioned, the laser is pulsed at the sample spot at frequencies of up to $10.000 \mathrm{~Hz}$ to generate ions. The sample plate is meticulously shifted after a fixed number of laser shots to prevent signal decrease due to sample depletion. Each shift thus presents "fresh" sample to the mass analyser. The pulsed light of the laser is absorbed by the co-crystalized matrix molecules. Following ablation, a phase explosion takes place in which clusters of analyte and matrix molecules fly away from the target towards the entrance of the mass analyser. At the same time, protons are transferred from the matrix to the (poly)peptide or vice versa to yield positively or negatively charged ions, respectively. Classical MALDI matrices such as $\alpha$-cyano-4-hydroxycinnamic acid (CHCA), sinnapinic acid (SA) and 2,5-dihydroxybenzoic acid (DHB) (Juhasz et al., 1993) typically yield singly charged ions. Other “cooler” matrices such as 1,5-diaminonaphthalene (1,5-DAN) (Fukuyama et al., 2006), 2,6-dihydroxyacetophenone (DHAP) (Gorman et al., 1996) and 5-aminosalicylic acid (5-ASA) (Sakakura and Takayama, 2010) matrices are available for MALDI, which 
allow the generation of multiply charged ions and can be used for in-source decay tandem mass spectrometry experiments.

In ESI, (poly)peptides in solution are passed through a capillary that ends in a narrow opening to produce small droplets (electrospray emitter). Ionisation is induced at atmospheric pressure by applying a distal voltage directly to the solvent or to the capillary outlet in which the mass spectrometer acts as ground, or by applying an electric potential difference between the mass spectrometer inlet capillary and a spray cap. During ESI, protons are transferred from the solvent to the (poly)peptide or vice versa to yield positively or negatively charged ions, respectively. Desolvation occurs prior to entrance into the vacuum of the mass spectrometer to prevent loss of vacuum and coulomb explosions of droplets that contaminate the inlet capillary or ion optics of the mass spectrometer. Contrary to MALDI, ESI typically generates multiply charged ions for peptides and proteins.

The choice for MALDI or ESI ionisation depends on the sample, mass analyser and desired mass spectrometry experiment that needs to be performed. For instance, one should consider the mass and expected charge of the (poly)peptide in relation to the upper mass range limit of the mass analyser. If we would analyse a peptide of 5999 Da by MALDI using CHCA matrix on an ion trap with an upper mass range limit of $3000 \mathrm{~m} / \mathrm{z}$, the ion would not be detected since its mass to charge ratio likely is $\mathrm{m} / \mathrm{z} 6000[\mathrm{M}+1 \mathrm{H}]^{1+}$. On the other hand, a mass analyser with a higher upper mass range limit, such as a time-of-flight (TOF) analyser, would be able to detect the $z=1$ ion generated by MALDI. Conversely, if the peptide acquires 3 protons in ESI to form an ion of $m / z 2000.7[\mathrm{M}+3 \mathrm{H}]^{3+}$, it is detectable by the ion trap.

\subsubsection{Mass analysers}

Once ionisation and desolvation have taken place, ions are introduced into the high vacuum region of the mass spectrometer where ion optics focus and transport the ion beam to the mass 
analyser. A variety of mass analysers are currently used in proteomics, each of which has their own specifications, advantages and limitations. One such specification is the resolution of an instrument. In mass spectrometry, resolution is defined by the full width at half maximum (FWHM; $\Delta m$ at $50 \%$ height) of a spectral peak. Resolution for mass spectrometers is expressed as the mass resolving power (R) which is calculated by $m / \Delta m_{50 \%}$.

Quadrupole mass analysers scan through a selected $m / z$ range using oscillating electric fields to create a stable trajectory for ions of a specific $\mathrm{m} / \mathrm{z}$ ratio to reach the detector (Kero et al., 2004). Ions with a stable trajectory produce a signal that scales proportionally to the number of ions that reach the detector. A single spectrum is thus the combined product of a few up to tens of thousands of trajectories at scan speeds of up to $1000 \mathrm{Th} \mathrm{s}^{-1}$ and more. Here, the number of trajectories that are being used per spectrum depends on the resolving power of the instrument and selected mass range. Quadrupole analysers are the lowest-resolution analysers in proteomics and are mainly employed in tandem to selectively quantify ions of interest. Isotopic resolution can generally be achieved only for up to doubly charged ions. Still, quadrupoles are frequently employed in combination with other mass analysers, such as time-of-flight (TOF) or orbitrap mass analysers, to select ions of interest for multiple stage mass spectrometric analyses $\left(\mathrm{MS}^{\mathrm{n}}\right)$.

Ion trap mass analysers can be classified into two types that essentially follow the same principle: the 3D ion trap (Paul trap) (Brancia, 2006; March, 1997; McLuckey et al., 1994) or the 2D ion trap (linear ion trap) (Douglas et al., 2005). Conceptually, an ion trap is based on the inverse principle of a quadrupole analyser. First, ions generated at the ion source are trapped together within a small radius inside the trap in two or three dimensions using a radio-frequency quadrupolar field. Inert helium gas inside the ion trap removes excess energy from trapped ions to ensure a stable trajectory. Once trapped, potentials are adjusted to scan through the selected mass range by ejecting only ions of a specific $\mathrm{m} / \mathrm{z}$ ratio out of the trap 
towards a detector. Similar to the quadrupole analyser, a single mass spectrum is the product of a few up to tens of thousands of scans at scan speeds as high as $52.000 \mathrm{Th} \mathrm{s}^{-1}$. Modern ion traps are considered high-resolution mass spectrometers and may achieve sufficient resolution to resolve the individual isotopes of ions up to charge state $z=6$. Similar to the quadrupole analyser, ion traps can be combined with other mass analysers such as ion-cyclotronresonance or orbitrap mass analysers for $\mathrm{MS}^{\mathrm{n}}$ experiments. Some notable characteristics of ion traps are that slower scan speeds result in higher resolution mass spectra and that FWHM is constant throughout the mass range. In addition, ion traps are able to perform multiple consecutive stages of mass spectrometry, up to $11\left(\mathrm{MS}^{11}\right)$ on commercial instruments.

Time-Of-Flight (TOF) mass analysers (Mamyrin, 2001) first accelerate ions after which they drift according to their velocities through a free-field region, until they reach a detector located at the end of the flight tube. The time of flight that an ion requires to reach the detector after the initial acceleration depends on its mass to charge ratio. Ions with a low mass to charge ratio travel faster than ions with a high mass to charge ratio. It is important that ions acquire the same kinetic energy during acceleration to achieve a high-resolution mass spectrum. By now, different methods have been established to minimise the kinetic energy distribution during acceleration and its negative effect on resolution. Both MALDITOF and ESI-TOF instruments may be equipped with an ion mirror or "reflectron" to focus ions (Cotter et al., 2007). Ions drift into the reflectron where they are deflected by an electric field and repelled towards the detector. The reflectron is situated at the end of the flight tube and the detector is placed off-axis near the beginning of the flight tube. Ions with the same $\mathrm{m} / \mathrm{z}$ ratio but with higher kinetic energy enter the reflectron deeper than ions with lower kinetic energy. As a result, ions with higher kinetic energy travel a longer flight path to arrive at the detector at approximately the same time as ions with a lower kinetic energy. Another advantage is that a reflectron nearly doubles the flight path of ions without physically 
extending the flight tube, which also enhances resolution. TOF instruments with continuous ion sources such as ESI use “orthogonal extraction” of ions into the flight tube to reduce the kinetic energy dispersion during acceleration (Chernushevich et al., 2001). "Delayed extraction” is an alternative for TOF instruments with pulsed ion sources such as MALDI to minimise the kinetic energy dispersion (Brown and Lennon, 1995). Some unique features of TOF analysers include the potentially unlimited upper mass range limit in linear mode, spectra acquisition-rate independent resolution, constant ultra-high mass resolving power across the entire mass range (up to $\mathrm{R} \geq 80.000$ ), and highest possible dynamic range $\left(10^{5}\right.$ ).

Fourier Transform Ion-Cyclotron-Resonance mass analysers (FT-ICR) (Marshall et al., 1998) determine the mass to charge ratio of ions from their circular motion (detected frequency) when trapped in a high magnetic field. An ICR cell is located at the centre (bore) of a high magnetic field superconducting magnet, which is in the range of 3-21 Tesla (T) (Hendrickson et al., 2015). First, ions are injected into the ICR cell and trapped by applying a trapping voltage to the front and back plates. Once trapped, ions have a small radius circular motion perpendicular to the magnet field along the z-axis. Next, a radiofrequency wave emitted by the excitation plates raises the kinetic energy of trapped ions in order to increase the radius of their circular motion. This results in ions orbiting close to the walls of the ICR cell, which are equipped with excitation and detector plates. At each passage, an ion package induces a current on the detector plates. Ions with low mass to charge ratio will have a fast circular motion and are detected at a high frequency, whereas ions with a high mass to charge ratio will have a slow circular motion detected as a low-frequency signal. The excitation frequency wave has the same frequency as the ion in the cyclotron to allow resonance absorption of this wave. Importantly, all ions of the same $\mathrm{m} / \mathrm{z}$ need to be in phase or grouped together in an orbit to produce an induction current. If ions are located randomly along the orbit, the induced current will be null. In Fourier transform mass spectrometry all ions present 
in the cyclotron are excited at the same time by applying a rapid scan of a large frequency range. This enables simultaneous detection of all ions present in the ICR cell in a single measurement. As a result, a highly complex wave will be recorded which is then converted by Fourier transformation into a frequency-dependent intensity function from which the mass spectrum is obtained. FT-ICR instruments offer the highest possible spectral resolution that can be achieved by mass spectrometry, over $\mathrm{R} \geq 10^{7}$ (at $\mathrm{m} / \mathrm{z} 400$ using a 7T magnet; Bruker Daltonics, www.bruker.com). Resolution increases with longer spectra acquisition time and higher magnetic field strength. It is, however, important to note that resolution is not constant across the mass range, but is inversely proportional to $\mathrm{m} / \mathrm{z}$, which means that resolution decreases significantly at increasing $\mathrm{m} / \mathrm{z}$. To date, FT-ICR instruments provide the widest range of fragmentation methods available in commercial instruments for structural characterisation of ions.

Orbitrap or electrostatic ion trap analysers (Makarov, 2000; Perry et al., 2008) measure the frequency of oscillating ions along the central electrode of the trap from which the individual frequencies and intensities are derived via Fourier transformation of the broadband signal to produce the mass spectrum. An electrostatic ion trap is capable of achieving ultra-high resolution spectra (up to $\mathrm{R}=240.000$ at $\mathrm{m} / \mathrm{z} 200$ with isotopic fidelity (Thermo scientific; www.thermoscientific.com]), next to what is possible by FT-ICR. However, loss of resolution is inversely proportional to $(\mathrm{m} / \mathrm{z})^{1 / 2}$, which results in a less dramatic loss of resolution at increasing $\mathrm{m} / \mathrm{z}$ compared to FT-ICR MS.

As mentioned before, each mass analyser has its own advantages and limitations. In addition, there is a significant difference in acquisition and maintenance costs between mass analysers. It is therefore important to choose the best suitable mass analyser for a specific experiment to ensure optimal results at lowest possible costs. Table $1<$ Table $\mathbf{1}>$ summarises relevant system specifications for commercially available instruments (2015). 


\subsubsection{Peptide sequencing: Tandem mass spectrometry}

Although a mass spectrum contains highly informative data about (poly)peptides, one should realise that proteomics applications also require structural information about ions of interest. Hence, accurate mass and isotope distribution cannot differentiate between peptides with identical amino acid composition but different amino acid sequence. For example, peptides SHAPE and HEAPS are composed of the same amino acids and consequently have an identical mass and isotope distribution. Hence, structural information from which the amino acid sequence can be deduced is required to differentiate between them. To this end, most mass spectrometers can produce mass spectra of fragment ions that are generated in a fragmentation experiment on an isolated ion. Such mass spectrometry experiments are referred to as "tandem mass spectrometry", since the mass of the (poly)peptide is measured in a first round of mass spectrometry (MS or $\mathrm{MS}^{1}$ ), followed by a second round of mass spectrometry (MS/MS or $\mathrm{MS}^{2}$ ) in which the "precursor" ion is isolated, fragmented, and its fragment ions are analysed. Instruments that have the capability to perform higher order $\left(\mathrm{MS}^{\mathrm{n}}\right)$ experiments are also denoted "tandem mass spectrometers", even though they are not limited to only or two successive MS experiments per se; commercial ion traps can perform up to $\mathrm{MS}^{11}$ experiments. Tandem mass spectrometers are often "hybrid instruments" that combine different mass analysers for various stages of $\mathrm{MS}^{\mathrm{n}}$ experiments. Examples are ESI Q-TOF (Chernushevich et al., 2001) and LIT FT-ICR MS (Peterman et al., 2005; Syka et al., 2004) instruments that use an analytical quadrupole (Q) or linear ion trap (LIT) for the isolation or isolation and fragmentation of precursor ions, respectively.

Most tandem mass spectrometry experiments begin with the isolation of a precursor ion from a mixture of available ions inside the instrument. This isolation step ensures that exclusive fragment ions from a single ion species are recorded in the $\mathrm{MS}^{2}$ spectrum. Mixed 
$\mathrm{MS}^{2}$ spectra that contain fragment ions from multiple precursors complicate the interpretation of the spectrum severely, since any of the fragment ions might derive from different precursor ions. Following isolation, the precursor ions need to be fragmented in some way to produce sequence-specific fragment ions. For this sake, most fragmentation methods aim at producing a complete set of unique fragment ions for which the mass difference between the consecutive fragments corresponds with the known (adjusted) mass of a single amino acid residue. It is important to realise that each unique fragment ion is produced by a single peptide backbone cleavage of a single precursor ion. This means that instead of a single precursor ion, multiple precursor ions, i.e. with identical mass-to-charge ratio, are used to generate a single fragmentation spectrum. Thus, the term "precursor ion” refers to the total complement of identical ions that undergo fragmentation.

Cleavage of the peptide backbone may occur at three different sites between amino acid residues to form peptide fragments: alkyl carbonyl bond (CHR-CO), peptide amide bond (CO-NH), and amino alkyl bond (NH-CHR) cleavage. A widely accepted nomenclature for peptide fragment ions is shown in its concise form in Figure $3<$ Figure $3>$. This nomenclature was first introduced by Roepstorff and Fohlmann (1984) and later modified by Biemann (1990) to accommodate unambiguous annotation of fragment ion type and fragment order. Fragment ions that extend from the N-terminus of the (poly)peptide are referred to as a-, b-, or c-ions, whereas their complementary C-terminal fragment ions are called z-, y-, or x-ions. Thus, the numbering of the $\mathrm{N}$-terminal fragment ions starts at the $\mathrm{N}$-terminus and the numbering of C-terminal fragment ions starts at the C-terminus. Different fragmentation methods generally induce only one of the three possible peptide backbone bone cleavages. For example, collision-induced dissociation (CID) typically produces b- and y-ion series, whereas electron-transfer dissociation (ETD) produces c- and z-ions. A range of methods is available on modern instruments for the controlled fragmentation of (poly)peptide ions in the 
gas phase. It is beyond the scope of this review to discuss all available fragmentation methods and we will limit to the two most common fragmentation methods for (poly)peptides: CID and ETD.

To date, CID is the preferred fragmentation method for peptides because of its high efficiency, sensitivity and short duty cycle time. In CID, precursor ions collide with an uncharged gas atom- usually nitrogen or argon- by which the kinetic energy is partially converted to vibrational energy until a critical threshold is reached and the precursor ion breaks into two fragments (Seidler et al., 2010). CID is commonly used to analyse proteolytical peptides fragments of proteins (e.g. tryptic peptides) or small proteins. A significant limitation of CID is its relatively "slow" fragmentation reaction, which allows energised ions to fragment through their weakest links. This may severely limit the amount of amino acid sequence information in the fragmentation spectrum because only few fragment ions are produced. In addition, labile post-translational modifications (PTMs) may be difficult to analyse by CID (Zhou et al., 2011). Phosphorylated peptides are a notorious example. In this case, CID tends to induce a dominant neutral loss of the phosphoryl group. As a consequence, peptide fragmentation occurs with very low efficiency since most of the energy is dissipated by the cleavage of the phosphate ester bond. In addition, the neutral-loss reaction impairs the assignment of the phosphorylation site since the phosphoryl group is not attached anymore to any of the fragment ions.

ETD is a "fast" fragmentation method, which induces near-instant fragmentation of precursor ions. In ETD, electrons are transferred from a reagent anion to a multiple-charged precursor cation via ion/ion reactions. Upon capturing an electron, a multiply protonated peptide or protein is transformed into a hypervalent species. The resulting odd-electron species undergo fragmentation pathways driven by radical chemistry, where the $\mathrm{N}-\mathrm{C} \alpha$ backbone bonds are cleaved to generate c- and z-type fragment ions (Huang and McLuckey, 
2010). ETD generally provides more extensive sequence information for larger molecules and preserves labile modifications such as phosphoryl groups to allow assignment of the modification site (Zhou et al., 2011). Both CID and ETD can provide complementary information (Guthals and Bandeira, 2012) that can be employed to fully elucidate both the structure of the PTM as well as the amino acid sequence and modification site (Hogan et al., 2005). An example is the analysis of glycopeptides by CID and ETD (Fig. 4) < Fig. 4>. Here, CID (Fig. 4A) provides structural information on the glycan moiety, while the amino acid sequence and modification site is elucidated by ETD (Fig. 4B).

\subsubsection{Separation of complex samples: LC-MS/MS}

The high complexity of most samples does not allow for direct tandem mass spectrometry analysis by ESI-MS/MS or MALDI-MS/MS. In general, direct analysis of complex samples by mass spectrometry will only produce signals for the most abundant ions due to dynamic range limitations and ion suppression. The highest possible dynamic range of Q-TOF instruments $\left(10^{5}\right)$ is still orders of magnitude lower than the dynamic range of protein abundances in biological samples, which can vary over ten orders of magnitude in eukaryotic cells. In addition, significant ion suppression would take place during ionisation due to competition between (poly)peptides for ionisation efficiency. Finally, diverse peptides with identical mass but distinct amino acid sequence would be detected as a single spectral peak and would produce a mixture of fragment ions in tandem mass spectrometry experiments. Therefore, mass spectrometers are often connected to other separation methods that partition the (poly)peptides from the sample at which resolved peaks are fed online into the ion source. For (poly)peptides, liquid chromatography (LC) is by far the most convenient technique for hyphenation with mass spectrometry via an ESI source. Capillary electrophoresis (CE) has unique advantages over LC for the separation of peptides and proteins (Haselberg et al., 2013; 
Robledo and Smyth, 2014) but is less common. We will therefore focus on LC as separation technique. The combination of liquid chromatography with online tandem mass spectrometry analysis is referred to as LC-MS/MS. Out of available chromatographic separation methods, reversed phase (RP) chromatography is mostly applied because of its reproducibility, robustness, high separation efficiency and its compatibility with ESI. To maximise sensitivity, columns preferably have small inner diameters of $75 \mu \mathrm{m}$ or $100 \mu \mathrm{m}$ that require flow rates of only 150-300 nl/min (Gama et al., 2013; Sestak et al., 2015). These low flow rates minimise the dilution of trace level (poly)peptides during separation, but they also provide better signal to noise for detected analytes: the ionisation efficiency in ESI increases proportionally with lower flow rates (Gama et al., 2013). Selection of chromatographic particles depends on various factors such as the size of the (poly)peptide and operational back pressure of the columns that can be handled by the liquid chromatograph. In general, smaller diameter particles provide higher resolution at the expense of substantial increase of the backpressure in the system. Presently, particles of less than $2 \mu \mathrm{m}$ are available for the routine separation of (poly)peptides. Such small particles require newer generation liquid chromatographs that can withstand operational pressures of 10000 PSI (690 Bar) up to 15000 PSI (1034 Bar) (Sestak et al., 2015). Pore sizes for peptide separations are in the range of 100-300 $\AA$ and for protein separations pore sizes may range between 300 and $8000 \AA$. Correspondingly, RP columns for peptide or protein separations are packed with C18 or C4 materials, respectively. Because of the low flow rate compared to the relatively large injection volume, which is in the range of $0.1-20 \mu \mathrm{l}$, most nanoflow LC setups take advantage of trap columns to minimize the amount of time that would be needed to load the sample onto the column. For example, injection of a

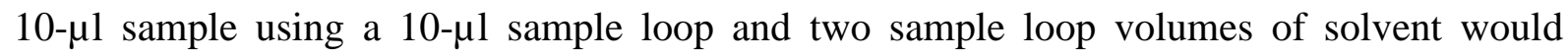
require at least 67 minutes of loading time at a flow rate of $0.3 \mu \mathrm{l} . \mathrm{min}^{-1}$, without taking the dead volume of the system into account. By comparison, applying the same injection and 
sample loop volumes, the loading time by a trap setup at a flow rate of $10 \mu \mathrm{l} . \mathrm{min}^{-1}$ is approximately two minutes.

\subsection{Protein identification}

In mass spectrometry, protein identification can be performed directly on the intact protein (top-down proteomics) (Catherman et al., 2014) or on peptides of the protein following enzymatic or chemical digestion (bottom-up proteomics) (Zhang et al., 2013). Bottom-up proteomics is by far the most common methodology thanks to its robust and sensitive performance, and its relatively low demands on instrumentation and experience with mass spectrometry technology.

\subsubsection{Peptide centric (bottom-up) proteomics}

From a generic perspective, three different approaches can be distinguished in bottom-up proteomics for protein identification: peptide mass fingerprinting, MS/MS database searches and de novo sequencing. In the first two methods, mass spectrometry data are matched to insilico calculated data for all available protein sequences in a database to find the proteins or peptides that best explain the experimental data. In peptide mass fingerprinting (Henzel et al., 2003), the collection of measured peptide masses is compared with the in-silico calculated peptide masses for each protein sequence and subsequently scored. The calculated protein identification scores determine which protein sequence(s) in the database provide the best, i.e. statistically significant, match with the experimental data. This method relies on mass accuracy of the measurement and sequence-specific proteolysis to assign protein identifications. Peptide mass fingerprinting is commonly performed on tryptic digests of (nearly) pure proteins in combination with MALDI-TOF MS. These (nearly) pure proteins are obtained by standard purification methods or after one or two-dimensional gel electrophoresis 
(1DGE, 2DGE) of a sample (see Section 3.1.1). The method is time demanding, but it is well suited for low-complexity samples of only a few proteins.

Contrary to peptide mass fingerprinting, MS/MS analysis allows the identification of a theoretically unlimited number of proteins in a sample through identification of the individual proteolytical peptides combined with protein database searches. As outlined above, in tandem mass spectrometry the mass of the proteolytical peptide can be determined from the acquired $\mathrm{MS}^{1}$ spectrum, whereas the sequence-specific fragment ion masses are derived from the $\mathrm{MS}^{2}$ spectrum. Database search algorithms first compare the precursor ion mass with those of all peptides from the in-silico digested protein sequences in the database to find a match with a specified mass tolerance. For each extracted peptide the algorithm will score the matching quality between the collections of measured and calculated fragment ions. The highest scoring (significant) spectrum-peptide match is considered as peptide identification. Finally, the collection of identified proteolytical peptides infers protein identifications. Figure 5 shows a schematic representation of the typical workflow in a LC-MS/MS based bottom-up (shotgun) proteomics experiment. <Fig. 5>. For database searches of $\mathrm{MS}^{2}$ spectra, various software programmes are available, such as MASCOT (Matrix Science) (Perkins et al., 1999), MaxQuant (Cox and Mann, 2008), Trans-Proteomic Pipeline (Pedrioli, 2010), X!Tandem (Craig and Beavis, 2004), SEQUEST (Eng et al., 1994), OMSSA (Geer et al., 2004) and PEAKS (Zhang et al., 2012). Each software package has its advantages and limitations, and a number of these has been critically evaluated by Dagda et al. (2010). Regardless of the software or database search algorithm used, care must be taken to control the false discovery rate (FDR) for both peptide and protein identifications when analysing large tandem mass spectrometry datasets (Aggarwal and Yadav, 2016; Brosch et al., 2009; Cox and Mann, 2008; Weatherly et al., 2005; Wright et al., 2012; Zhang et al., 2012). FDR is determined by performing a search against the protein sequence database and a decoy sequence database that 
contains nonsense protein sequences. Any spectrum-peptide match in the decoy database is considered a false positive result. The false discovery rate is then controlled by adjusting thresholds to include spectrum-peptide matches until the maximum-allowed FDR is reached (typically 1\% ).

Database searches to identify peptide and protein sequences are not always possible or may not retrieve a significant spectrum-peptide match, irrespective of the data quality. The finding of a match between a peptide sequence and a fragmentation spectrum presumes that the measured peptide sequence is actually available in the database. This is obviously a problem in proteomics of species for which there is no comprehensive genomic sequence available. The application of de novo sequencing provides an alternative to database search strategies as the amino acid sequence is determined directly from the $\mathrm{MS}^{2}$ spectrum (Seidler et al., 2010). Here, the mass difference between consecutive fragment ions establishes the amino acid sequence as shown in Figure 6 for peptide "YIC(СAm)DNQDTISSK" from BSA. $<$ Figure 6 $>$. The annotated deconvoluted spectrum reveals a dominant $y$-ion series from which part of the peptide sequence can be read directly. This spectrum is easily interpreted, but manual interpretation of fragmentation spectra is challenging and time-consuming in general since each spectral peak in the $\mathrm{MS}^{\mathrm{n}}$ spectrum can be any of the expected fragment ion types (e.g. b- or y- ion). The processing of large tandem mass spectrometry datasets is facilitated by de novo sequencing software programmes (Allmer, 2011), including PEAKS (Zhang et al., 2012), Lutefisk (Taylor and Johnson, 1997), PepNovo (Frank and Pevzner, 2005), NovoHMM (Pevtsov et al., 2006), AUDENS (Grossmann et al., 2005), Novor (Ma, 2015), Sherenga (Dancik et al., 1999), UniNovo (Jeong et al., 2013) and pNovo ${ }^{+}$(Chi et al., 2013). In these programmes, high-throughput automated interpretation of $\mathrm{MS}^{2}$ spectra is supported by different algorithms. Applicability of these programmes specifically depends on the type of mass analyser used to generate tandem mass spectrometry data as concluded by 
Pevtsov et al.(2006), who compared the performance of established de novo sequencing algorithms for Q-TOF and Ion trap tandem mass spectrometry data.

\subsubsection{Protein centric (top-down) proteomics}

The direct analysis of intact proteins by mass spectrometry, referred to as "top-down" proteomics, is preferred over peptide centric methods when information about the exact molecular composition of a protein ("proteoform”) is required. The term proteoform was proposed by Smith, Kelleher and colleagues (2013) to designate all of the different molecular forms in which the protein product of a single gene can be found, including changes due to genetic variations, alternatively spliced RNA transcripts and PTMs. In bottom-up proteomics, information on proteoforms in a sample is irreversibly lost after digestion of a protein into smaller peptide fragments (Rappsilber and Mann, 2002). Analysis of intact proteins by topdown proteomics preserves the true biological complexity of proteins since every unique proteoform is analysed directly "as is” (Tipton et al., 2011; Catherman et al., 2014). One may wonder why bottom-up approaches are still generally used in proteomics, if top-down proteomics is capable of providing exact information at the level of proteoforms. The answer to this question relates to the fact that top-down proteomics is very challenging to perform due to the high complexity of the mass spectral data and technical limitations (Smith et al., 2013). In general, top-down proteomics is still unable to achieve the high sensitivity and throughput that bottom-up proteomics provides. The application of bottom-up approaches is thus preferred in research that does not require proteoform specific information. However, recent hardware and software developments have been making a start to close the gap between topdown and bottom-up proteomics with respect to sensitivity, throughput and robustness. The lower sensitivity for whole proteins as compared to peptides in LC-ESI MS ${ }^{\mathrm{n}}$ experiments supported by modern instruments is partly explained by "charge state dilution" and "isotope 
dilution” effects. Unlike peptides, a single protein species typically acquires a variable number of charges during ionisation by ESI. This implies that the total signal for a single protein is divided over all the different charge state peaks in the mass spectrum ("charge state dilution”), as observed in Figure 2A. In addition, proteins have a broad isotopic distribution that again lowers the signal to noise ratio (“isotope dilution”). An example of such isotopic distribution pattern for proteins is presented in Figure 7 showing a deconvoluted mass spectrum of horse cytochrome $c(12.3 \mathrm{kDa}) .<$ Fig. $7>$. One may note that the monoisotopic peak for cytochrome $c$ is barely detectable and that the monoisotopic peak may not be observed in deconvoluted spectra of larger proteins. In fact, the monoisotopic mass is calculated by a peak-detection algorithm (SNAP 2.0, Bruker Daltonics), which fits an in silico isotopic distribution model, based on the average amino acid "averagine”, to the experimental data. The high complexity of data in top-down proteomics generally requires ultra-high resolution mass spectrometry in combination with sophisticated peak picking and/or deconvolution algorithms to produce information-rich data. Thus far, top-down proteomics primarily relied on FT-ICR and electrostatic ion trap mass analysers. However, the latest generation of ultra-high resolution Q-TOF instruments provide an interesting alternative to FT-based mass analysers. These new Q-TOF spectrometers have unique characteristics, such as a significantly higher dynamic range, wider mass range and constant mass resolving power over the full mass range (Table 1).

Protein identification in top-down proteomics by tandem mass spectrometry is in essence similar to bottom-up proteomics. Data base searches supported by software algorithms permit the identification of proteoforms, but de novo sequencing can be applied as well. However, database search strategies are adapted for top-down proteomics, since any possible combination of PTMs as well as sequence variants need to be considered to successfully match an acquired fragmentation spectrum to a specific proteoform. Software 
programmes capable of handling top-down proteomics data include ProSightPTM2 (LeDuc et al., 2004; Zamdborg et al., 2007), MASCOT top-down (Karabacak et al., 2009) and MSAlign+ (Liu et al., 2012). CID or ETD fragmentation methods are both applicable in $\mathrm{MS}^{\mathrm{n}}$ experiments on whole proteins. Generally, ETD fragmentation will provide higher sequence coverage than CID, but the former method requires more precursor ions to produce reasonable signal to noise $\mathrm{MS}^{\mathrm{n}}$ spectra.

Top-down proteomics commonly find application in mass spectrometry measurements on intact proteins in their denatured state. Developments in "native mass spectrometry" will enable the study of intact proteins as well, and even of protein complexes in which the higher order complexity is preserved. Eventually, native mass spectrometry may allow the most detailed analysis of protein complexes composed of unique combinations of proteoforms that form distinct complexes together. The opportunities of native mass spectrometry is illustrated by an experiment with Trastuzumab (Figure 8). <Fig. 8>. Trastuzumab is a therapeutic monoclonal antibody and it is a protein complex composed of two heavy and two light chains (Bernard-Marty et al., 2006). Each heavy chain may be substituted by different N-glycans (Listinsky et al., 2013); the light chains are present as one proteoform. The different combinations of heavy chain proteoforms in a single antibody molecule yield unique protein complexes of distinct masses, except for combinations of N-glycans with identical masses (e.g. G0F/G2F and G1F/G1F combinations in Figure 8). The multiple spectral peaks in the deconvoluted mass spectrum of Trastuzumab result from the unique combinations of heavy chain proteoforms. Within the context of this review, it should be noted that native mass spectrometry is still notoriously challenging for membrane complexes.

\subsubsection{Protein quantitation: Principles}


As mentioned previously, the intensity in acquired mass spectra correlates with the abundance of a (poly)peptide in the sample. However, intensities for equimolar amounts of different peptides may vary over several orders of magnitude in mass spectra due to differences in ionisation efficiency. Alternatively, the integrated peak area of a peptide eluting from the LC column serves as a measure for its abundance. Spectral intensity and peak area are thus meaningful to determine the relative abundance of a chemically identical (poly)peptide between samples. However, this data are of less value in making quantitative comparisons between different (poly)peptides. An exception to this rule holds for stable isotope labelled variants of the same peptide.

In profiling experiments, the chromatographic peak area of extracted ion chromatograms from $\mathrm{MS}^{1}$ spectra is used to compare protein abundances between samples. Quantitation software programmes for shotgun proteomics such as MaxQuant (Cox and Mann, 2008), MASCOT Distiller (Matrix Science; www.matrixscience.com), MSQuant (Gouw and Krijgsveld, 2012), TPP (Pedrioli, 2010) or IDEAL-Q (Tsou et al., 2010) extract MS $^{1}$ intensity-based quantitative information for identified peptides into LC-MS/MS datasets. Most of these programmes accurately match mass and retention time to robustly retrieve quantitative information for peptides that were not identified (but present) in some of the parallel measurements. This is particularly important in shotgun proteomics where precursors are on-the-fly and data-dependently selected by the mass spectrometer for subsequent $\mathrm{MS}^{2}$ experiments. Since part of the precursors selected for $\mathrm{MS}^{2}$ analysis differ between measurements, data gaps are introduced for proteins that were present in a sample but were excluded from $\mathrm{MS}^{2}$ analysis. To minimise those data gaps, software programmes construct a database that contains the accurate mass and retention time for every identified peptide within a proteomics experiment to subsequently find a peptide ion in the $\mathrm{MS}^{1}$ spectra of every individual measurement without the need for a positive peptide identification. 


\subsubsection{Label-free and stable isotope labelling quantitation methods}

For intensity-based quantitation in shotgun proteomics both label-free and stable isotope labelling quantitation methods have been developed. In label-free quantitation, each sample is analysed in a separate LC-MS/MS run at which the intensity of a peptide from each run is used to calculate the relative abundance of proteins between two or more samples. Data are normalised to correct for experimental artefacts that may result from differences in, for instance protein amount or sample injection volume.

The so-called spectral count methods provide an alternative to intensity-based quantitation (Lundgren et al., 2010). In spectral counting, the number of $\mathrm{MS}^{2}$ spectra that are matched to a protein sequence are taken as a measure of its abundance. Spectral count methods have much lower accuracy and linear dynamic range than intensity-based quantitation methods, but quantitative data are easily calculated from experimental database search results. A common spectral count procedure to assess protein abundance differences between samples uses the exponentially modified protein abundance index (emPAI) (Ishihama et al., 2005). The emPAI value is calculated by the following formula: emPAI = 10

$\wedge$ [ $\left.\mathrm{N}_{\text {observed peptides }}\right] /$ [ $\left.\mathrm{N}_{\text {observable peptides }}\right]$. Where $\mathrm{N}_{\text {observed peptides }}$ is the number of unique peptide matches or total number of matched $\mathrm{MS}^{2}$ spectra for a given protein sequence and $\mathrm{N}_{\text {observable }}$ peptides is the number of unique observable peptides from an in-silico digest of the protein sequence. Since emPAI calculations compensate for the number of detectable tryptic peptides between protein sequences, emPAI values allow comparisons between different proteins.

Stable isotope labelling of peptides can be achieved by either in-vitro or in-vivo labelling approaches. Common in-vitro labelling methods include stable isotope dimethyl labelling (Boersema et al., 2008, 2009), isotope coded affinity tags (ICAT) (Gygi et al.), tandem mass tags (TMT) (Thompson et al., 2003), or isobaric tags for relative and absolute 
quantitation (iTRAQ) (Ross et al., 2004). By the introduction of labels that differ in isotope composition $\left({ }^{1} \mathrm{H}\right.$ vs. ${ }^{2} \mathrm{H},{ }^{12} \mathrm{C}$ vs. ${ }^{13} \mathrm{C},{ }^{14} \mathrm{~N}$ vs. ${ }^{15} \mathrm{~N},{ }^{16} \mathrm{O}$ vs. $\left.{ }^{18} \mathrm{O}\right)$, a mass offset is realised for peptides between two or more samples. This allows samples to be mixed together and analysed in a single LC-MS/MS experiment using the mass offset to differentiate between the samples ("multiplexing”). Importantly, the different isotope-labelled versions of a peptide exactly co-elute in reversed-phase liquid chromatography, enabling unambiguous relative quantitation. Stable isotope dimethyl labelling and ICAT allow multiplexing of 2 to 3 different samples at which the signal intensity in $\mathrm{MS}^{1}$ spectra serves quantitation. Dimethyl labelling is the most popular in-vitro method due to its simple, efficient, and robust derivatisation of peptides at very low cost. Also TMT and iTRAQ have gained rapidly increasing interest. TMT and iTRAQ reagents bind $\mathrm{N}$-terminal amine groups (and the $\varepsilon$ amine of lysine residues) of all peptides. In contrast to dimethyl and ICAT labelling, TMT and iTRAQ reagents are isobaric, being composed of a reporter molecule (of variable mass) combined with a balancer (also of variable mass) such that the molecular masses of reporter and balancer remain the same in the different reagents. During the tandem MS step, the bound iTRAQ label fragments, at which the low-mass reporter is used for peptide quantitation, whereas the remaining peaks in the MS/MS spectrum serve peptide identification. By the constant mass of the labels, the complexity of the sample is not increased, which allows for multiplexing of up to 10 different samples in a single analysis (McAlister et al., 2012; Werner et al., 2014). Here, the ratio between the various reporter ions in the $\mathrm{MS}^{2}$ or $\mathrm{MS}^{3}$ spectrum is used for relative quantitation.

For in-vivo metabolic labelling of (poly)peptides with stable isotopes, cells are grown in the presence of stable isotope-coded amino acids (SILAC) (Ong et al., 2002). This method requires organisms that are auxotrophic for the pertinent amino acid or organisms in which its biosynthesis is fully arrested, when supplied with the labelled amino acid. If not available, 
${ }^{15} \mathrm{~N}$-ammonium may serve as the substrate for the in vivo labelling (Krijgsveld et al., 2003; Wang et al., 2002).Care must be taken to achieve near complete metabolic incorporation of the stable isotopes to avoid labelling artefacts (unless pulse labelling is applied to study protein synthesis or protein degradation kinetics).

Label-free quantitation and stable isotope labelling approaches have their own advantages and limitations. First of all, the multiplexed analysis of 2-10 samples in a single LC-MS/MS analysis effectively removes run-to-run variation in signal intensities. In case of in-vivo isotope labelling, cells can even be mixed prior to protein extraction to eliminate any experimentally introduced variation between samples. Label-free quantitation on the other hand does not increase the complexity of the sample and permits the (retrospective) use of LC-MS/MS data from different experiments.

There is growing interest in the field of proteomics to acquire multiplexed tandem mass spectra using data-independent analysis (DIA). In DIA, all precursor ions within the full $\mathrm{m} / \mathrm{z}$ range, or in multiple overlapping narrow $\mathrm{m} / \mathrm{z}$ windows of f.i. $25-50 \mathrm{Th}$, are fragmented at the same time and fragment ions measured by high-resolution mass analysers. Different approaches are subsequently used to deconvolute the multiplexed $\mathrm{MS}^{\mathrm{n}}$ spectra as to enable identification and quantitation of co-fragmented peptides. DIA is used to bypass the technical limitations of tandem mass spectrometry instruments to ultimately identify and quantify the entire proteome. DIA is still in development and considering its main application (proteome wide analysis) is not key to analyse ETC complexes, we have confined ourselves to datadependent analysis (DDA) methods that rely on precursor isolation for $\mathrm{MS}^{\mathrm{n}}$ experiments. If interested, readers are encouraged to read the excellent review on DIA methodology by Chapman et al. (2014).

\subsubsection{Untargeted and targeted approaches}


The above quantitation methods are employed in profiling experiments in which up to thousands of proteins are analysed in an untargeted fashion. Unfortunately, proteins of interest can go beyond detection and quantitation due to technical reasons such as the quasi-random nature of precursor selection for $\mathrm{MS}^{2}$ analysis by the mass spectrometer or overlapping isotope patterns of co-eluting peptides. This is a major limitation for untargeted experiments. Another disadvantage of untargeted measurements is that statistical analysis and biological interpretation of acquired data can be highly challenging for large datasets. In addition, why should one measure thousands of proteins if only data from a handful of a priori known proteins is needed to test a hypothesis?

Targeted proteomics offers an attractive alternative to profiling in which only a subset of peptides from proteins of interest are measured selectively (Gallien et al., 2011; Gillette and Carr, 2013). Advantages of targeted proteomics over profiling methods are its superior sensitivity, robustness, higher sample throughput (by using short LC gradients), and simplicity of the data. Targeted proteomics is usually performed on triple quadrupole mass spectrometers (QqQ) using “multiple reaction monitoring” (MRM). In selected reaction monitoring (SRM) on QqQ instruments, a precursor ion is selected by the first quadrupole, dissociated in the second quadrupole, and one of the generated fragment ions is exclusively selected by the third quadrupole for detection. The precursor-ion and fragment-ion pair is referred to as SRM “transition". Ideally, a transition is selected to be unique for a single peptide to allow straightforward quantitation via a single chromatographic peak in its constructed SRM chromatogram. Unfortunately, it is often impossible to find a single transition that is unique for a given peptide in highly complex peptide mixtures. The problem of non-specific transitions is solved by MRM. In MRM, multiple fragment ions of one precursor ion are measured by separate SRM measurements. Although each separate transition might detect several peptides, leading to multiple peaks in its chromatogram, the 
combination of transitions is chosen to be specific for only a single peptide. Therefore, the correct chromatographic peak in each SRM chromatogram that corresponds with the peptide of interest is readily identified by searching for a chromatographic peak with the exact same retention time and peak shape in all SRM chromatograms of the target peptide. Different software programmes are available for the selection of protein specific peptides with optimal quantitative characteristics and subsequent selection of MRM transitions (Colangelo et al., 2013). The software programme Skyline (MacLean et al., 2010b) is at present the most commonly used software tool to develop MRM assays in proteomics. It also supports empirical refinement of SRM methods to achieve optimal quantitative results (Bereman et al., 2012; Maclean et al., 2010a). Absolute quantitation is also possible by mixing a known amount of synthesised stable isotope-labelled peptide standard (AQUA peptide; Stemmann et al., 2001) into the sample prior to injection. The mass spectrometer is set to acquire MRM data for both the native ("light”) and stable isotope labelled ("heavy”) versions of the peptide such that the absolute amount of the native peptide can be calculated on the basis of an $a$ priori-prepared standard curve of the heavy standard peptide.

\section{BACTERIAL ELECTRON TRANSFER CHAINS AND THEIR REGULATION: GLOBAL APPROACHES}

In principle, taking advantage of the power of proteomics, the full protein complement of a bacterial cell can be analysed and quantified in a single experiment. However, practice is more refractory for several reasons: the enormous complexity of even relatively "simple" microorganisms; physicochemical properties of proteins that pose specific demands as to their detection and identification; differences in the abundance of the different protein species that can vary over six orders of magnitude, and efforts and costs associated with MS analyses and 
data handling. In the next Section 3.1 we will cover these aspects in a general way, starting from two approaches that are aimed at analysing proteomes from the global, discovery-based perspective, (1) proteomics supported by two-dimensional gel electrophoresis (2DE) (Section 3.1.1) and (2) shotgun proteomics (Section 3.1.2). Hereafter, we will continue with membrane proteins, a subset of proteins of which proteomic analyses are challenging, but that are key components in bacterial ETS (Section 3.2). We will close this part of our review with an outline of proteomic methods to quantify protein abundance and differences in this, which is essentially the study of differential expression, both at the translational and post-translational levels (Section 3.3).

\subsection{DE-based methods and shotgun proteomics}

\subsubsection{Methods based on 2D IEF SDS-PAGE, the classical approach}

The success of this method was founded on two major experimental breakthroughs: 2DE separation of large numbers of proteins (O’Farrell, 1975; Neidhardt, 2011) and their identification by MS (Hillenkamp et al., 1990, 1991; Henzel et al. 1993; James et al. 1993; Pappin et al., 1993; Yates $3^{\text {rd }}$ et al. 1993). Separation relies on two unrelated properties of a protein, its isoelectric point $(\mathrm{p} I)$ and molecular mass. Upon electrophoresis through a $\mathrm{pH}-$ gradient gel, a protein in a mixture migrates until it reaches that part in the gel that equals its $\mathrm{p} I$ value. A prerequisite for the proper separation is the presence of isoelectric focusing (IEF) gels or gel strips with stable, immobilised $\mathrm{pH}$ gradients (IPG). Gel strips that either cover a wide range of $\mathrm{pH}$ values ( $\mathrm{pH} 3-10$ ) or that are more narrow-ranged (gradients across 2, 1, 0.5 or even less than $0.5 \mathrm{pH}$ units) are commercially available. The latter enables the better resolution of complex protein mixtures in the $\mathrm{pH}$ range applied. Following IEF, the gel strip is positioned unto a large (e.g. $20 \times 20 \mathrm{~cm}$ ) denaturing gel and proteins are separated in the second dimension by SDS polyacrylamide gel electrophoresis (SDS-PAGE), based on their 
molecular masses. This technique enables the separation of several thousands of proteins from a sample on a single gel. Next, proteins are visualised by staining, and proteins of interest can be individually picked and digested by a proteolytic enzyme (usually trypsin) or a cocktail of proteolytic enzymes. Cleaved proteins are then analysed by MALDI-TOF MS, fast atom bombardment (FAB) or electrospray ionisation (ESI) MS. Proteolytic cleavage results in a mixture of peptide fragments that is unique for a certain protein (“fingerprint”). The comparison of this fingerprint against an in silico-generated peptide database derived from the genome of the organism under investigation allows the identification of the analysed protein with a certain amount of confidence (Sections 2.1.2 and 2.2.1). Thus, this technique of peptide mass fingerprinting starts from the complete and resolved polypeptide, unlike the shotgun approach described below.

The 2DE-MS methodology has been applied to many prokaryotic species. Laboratories have developed all sorts of variations on basic techniques in order to improve proteome analysis of their model organisms, often consisting of species-specific preparation of the protein samples. This methodology has been used for whole-cell proteomics as well as for the proteomic analyses of subcellular fractions, such as the cytoplasmic proteins, the protein complement associated with the cytoplasmic membrane, with the cell surface outer membrane in Gram-negative bacteria or the cell envelope of Gram-positive species. All these aspects have been described in numerous publications and have been covered in reviews in which the merits and future perspectives of 2DE-MS are critically evaluated (e.g. Cordwell, 2004; Görg et al., 2004; Issaq and Veenstra, 2008; Mathy and Sluse, 2008; Brewis and Brennan, 2010; Chevalier, 2010; Rabilloud et al., 2010; Curreem et al., 2012). Here, we would like to illustrate the opportunities and intrinsic shortcomings of 2DE-MS with our work on Methanothermobacter thermautotrophicus strain $\Delta H$ (Farhoud, 2011). 
M. thermautotrophicus is a methane-forming (methanogenic) Archaeon that gains its energy for autotrophic growth from the oxidation of hydrogen with $\mathrm{CO}_{2}$ as the terminal electron acceptor. It was one of the first organisms whose genome was sequenced (Smith et al., 1997). This genome codes for 1922 open-reading frames (ORFs) and comprises 1874 protein-coding sequences, which that had been divided over 49 functional categories next to three categories with unknown function (unclassified, conserved and unknown, hypothetical proteins). At the time of publication (1995), 844 of these protein-coding ORFs could be given an annotated function (46\%), a situation that still holds in the genomic database (NCBI accession number, PRJNA289). In the years that followed the publication of the genome, our knowledge on protein functions has advanced greatly, and keeping up with literature, we now can assign a function to 1594 protein ORFs (83\%) (Farhoud, 2011). To get a better understanding of which genes are expressed at the protein level and under which conditions, we performed a comprehensive 2DE analysis, employing a variety of IEF gradient gels. An example of this is shown in Fig. $9<$ Fig. 9>.

Figure 9 immediately reveals the strength of 2DE analysis, visibility in what one is doing, but this strength is a weakness at the same time. After gel electrophoretic separations and gel staining, over 3000 protein spots were analysed by MALDI-TOF MS, which were unambiguously identified with a > 95\% success rate: “you can get whatever you can see”. However, the drawback is: “you won’t get what you don’t see”. Small-sized proteins $(<10$ $\mathrm{kDa}$ ) generally went unassigned, mainly due to the lack of sufficient and appropriate proteolytic cleavage sites for reliable identification. To quite an extent, successful MSsupported identification depends on high-quality genome assembly of the organism under investigation and its high-quality annotation. No matter how trivial, these factors may compromise future proteomic studies based on metagenomes that have been assembled and annotated automatically without proper, laborious and time-demanding curating by experts. 
For instance, even though it was well assembled, we noticed that in the $M$. thermautotrophicus genome 34 pairs of adjacent ORFs that were translated into N-terminal and C-terminal parts of known or conserved proteins. By single nucleotide substitution, deletion or insertion all pairs could be merged into full-length proteins. Six of these pairs comigrated into the same spot upon 2DE (see f.i. Fig. 9B), indicating that the gene splitting was rather the result of sequencing errors than of genetic events.

Protein staining also provides the means for protein quantification. In 2D gels this can be done by gel scanning combined with software programs that convert the size of a spot and its colour intensity into a protein amount. However, like in each protein staining method the practical applicability depends on two factors, detection limit and the dynamic range in protein amounts, expressed as log values, giving a linear response. Conventional staining dyes such as Coomassie Brilliant Blue (CBB), colloidal Coomassie and silver nitrate have detection limits of 50, 8-16 and 0.5-4 ng of protein, respectively (Curreem et al., 2008; Chevalier, 2010). (Note that only pico- or femtomoles of protein are needed for MS analysis). The dynamic range of the more sensitive silver staining is very low (1.5 log) and this method is not easily compatible with MS. As alternatives, differently fluorescent dyes with detection limits in the nanogram range, like Sypro Ruby, Flamingo and Deep Purple, have been developed (Poland et al., 2005). Albeit not particularly sensitive, the advantage of these fluorescent dyes is their dynamic range (4 log). Furthermore, proteins can be selectively stained on basis of the presence of haem groups or iron (Ferrer et al., 2007), which could be of relevance in studies on respiratory complexes. Nevertheless, lowly expressed proteins will go unseen (and undetected) in 2DE. In addition, such proteins can be obscured in the gels by highly expressed proteins. The use of narrow-ranged IEF strips may obviate the latter problem to some degree (Fig. 9B). 
In 2DE gels, a protein species may be represented by multiple spots that mainly differ in their $\mathrm{p} I$ values (proteoforms, “trains”). Proteoforms could be the result of physiological relevant PTMs (phosphorylation, substitution of charged amino acids with other groups), but this is not necessarily the case. Proteoforms also might represent folding isomers or other electrophoresis artefacts (Nag et al., 1994; Lutter et al., 2001; Berven et al., 2003; Paton et al., 2008). In Fig. 9, several examples of protein trains in the proteome of $M$. thermautotrophicus are highlighted. Two such examples are the alpha subunits of methaneforming methylcoenzyme $\mathrm{M}$ reductase type I (McrABC) and type II (MrtABC) that were observed as 3-5 proteoforms (Fig. 9B). The high-resolution crystal structure of Mcr I, indeed, reveals 5 PTMs in this alpha subunit, being methylated amino acid residues in close proximity to the $\mathrm{F}_{430}$ catalytic site (Ermler et al., 1997; Graberse et al., 2001). 2DE suggests various proteoforms, but a directed MS method would be needed to substantiate if these proteoforms are related to different degrees of methylation. In general, PTMs easily evade identification because modified peptides are hardly ionised by MALDI, peptide coverage of in-gel trypsin digestion is relatively low, substitution of amino acids may go unnoticed in database searches and phosphopeptides, for example, tend to decompose prior to detection the MALDI-TOF reflector mode. The identification of PTMs requires its dedicated MS analysis methods (Section 4.2).

Altogether, 2DE of M. thermoutotrophicus cell extracts allowed the identification of 633 unique proteins (33.8\% of the proteome), including 50 out of 109 proteins (45.9\%) involved in the process of methanogenesis. However, recovery was biased. High-molecular weight proteins (MW> $50 \mathrm{kDa})$ and basic proteins (pI>10) were not well-represented in the dataset, which is due to the poor resolution properties of IEF for such proteins. Most notably, only 26 out of the predicted 406 membrane-bound proteins (6.4\%) could be found. Apart from other reasons discussed in Section 3.2.1, the low yield of membrane-bound proteins is 
caused by their insolubility: these proteins precipitate in the IEF gel before reaching their pI (Braun et al., 2007).

The above considerations do not apply to M. thermautotrophicus specifically, but hold in general ways for 2DE proteomic analyses (see the reviews cited above). Apart from that, the method is quite laborious and time-demanding. Still, 2DE-MS could be very well the method of choice to address research questions with a more limited and focused scope than the analysis of the total proteome of a prokaryote.

\subsubsection{Shotgun proteomics}

To overcome the limitations of 2DE-based proteomics, shotgun proteomics, an alternative method that allows the high-throughput analysis of complex protein samples has been implemented (Washburn et al., 2001; Wolters et al., 2001; Aebersold and Mann, 2003). In this method, proteins in a sample are proteolytically cleaved before tandem MS. Next, all peptide fragments are computationally matched to the protein sequences in the database (see Section 2.2.2) (Washbur, 2004; Wei et al., 2005; Yates et al., 2009). Before MS analysis, the peptide mixture is resolved by reversed-phase (C18) liquid chromatography (LC) on a nano capillary column that is connected on-line with the tandem mass spectrometer (LC-MS/MS). This whole procedure is fully automatized and to achieve a better resolution, LC can be performed in two or even more dimensions (MudPIT) by use of strong cation-exchange, immobilised metal affinity or $\mathrm{TiO}$ columns placed before the hydrophobic column (Whitelegge, 2002; Kislinger et al., 2005; Sun et al., 2008; Block et al., 2009; Fränzel and Wolters, 2011; Cheung et al., 2012). For further reduction of the sample complexity, proteins can be pre-separated by conventional SDS-PAGE or liquid chromatography. After SDSPAGE, the gel is cut into multiple pieces, proteins in the pieces are digested in-gel, and digests are subjected to LC-MS/MS separately. This pre-separation may also involve off-line 
IEF, common protein separation methods such as anion-exchange or affinity column chromatography, capillary electrophoresis, as well as methods that have been specifically designed for tandem MS proteomic analyses (Lam et al., 2007; Cologna et al., 2010; Gilmore and Washburn, 2010; Freeman and Ivanov, 2011; ). Obviously, this method can be applied to lysates of whole cells as well as of subcellular fractions, which have been enriched or fully purified by designated procedures. A variety of in vitro and in vitro labelling methods is then available to quantify relative or absolute protein amounts, as described in Section 2.3.2.

Shotgun proteomics has been broadly used to make an inventory of the proteome of bacterial model species such as the Gram-negative Escherichia coli (Han and Lee, 2006; Krug et al., 2013), Gram-positive Bacillus subtilis (Völker and Hecker, 2005; Wolff et al., 2007; Becher et al., 2011), the industrially relevant Corynebacterium glutamicum (Fränzel et al., 2010; Fränzel and Wolters, 2011), pathogens such as Streptococcus pneumoniae (Sun et al., 2011), Mycobacterium tuberculosis (Mawuenyega et al., 2005; de Sousa and Wiker, 2011; Calder et al., 2015) and Mycoplasma pneunomiae (Catrein and Herrmann., 2011), environmentally relevant Shewanella oneidensis (Elias et al., 2005; Brown et al., 2010), the extreme thermophilic Archaeon Pyrococcus furiosis (Lee et al., 2009) and various others. As the result of extensive analyses of whole-cell lysates or subcellular fractions of these organisms, 50-60\% of all proteins encoded in their genomes could be recovered. In case of the “relatively simple” Mycoplasma pneunomiae, no less than 620 gene products out of the 700 protein-coding genes (89\%) could be identified (Catrein and Herrmann., 2011). Here, tandem affinity chromatography was extensively utilised to selectively enrich proteins from the cytoplasmic protein complement. Quite recently, a similar recovery (88\%, viz. 2300 out of the approximately 2600 protein-encoding ORFs) has been established for E. coli by the application of high-resolution MS combined with Super-SILAC protein quantification (Soares et al., 2013; Soufi et al., 2015). By applying LC-MS/MS combined with SDS-PAGE pre- 
fractionation, we could identify 894 unique proteins (48\%) from the soluble and membrane fractions of $M$. thermautotrophicus grown under high and low concentrations of its energy source hydrogen (Farhoud, 2011). Of these 894 proteins, 372 were not found in the 2DE proteome, whereas 111 proteins retrieved by the latter method were not detected by the shotgun approach. Apparently, both approaches have their flaws. Together, both methods allowed the identification of 1005 different polypeptide gene products (54\% of the expected proteome), including 83 proteins (76\%) involved in primary methanogenic metabolism. Importantly, LC-MS/MS gave a far better yield of membrane proteins (124; 30\%); still this type of proteins remained underrepresented in the dataset. Indeed, most of the proteins of the methanogenesis pathway that remained beyond detection were membrane proteins. In line herewith, proteins that were obtained with $<50 \%$ efficiency belonged to functional categories dominated by membrane proteins, viz. glycerolipid metabolism (10 out of 22 expected proteins; 45\%), cell envelope and membrane metabolism (51/129; 39.5\%), and transport of inorganic (47/95; 49.5\%) and organic (12/38; 31.5\%) compounds. Obviously, these percentages may be underestimations, since only part of the genome may be expressed under the conditions at which $M$. thermautotrophicus was grown. Next to this, membrane proteins might have been observed, but with too low peptide scores to permit reliable identification.

It goes without saying that shotgun proteomics has definite benefits above classical 2DE-MS, especially regarding its opportunities for high throughput analysis. Nevertheless, the former method has its limitations. No matter how welcomed, the comprehensive in-depth analysis of the flood of data is timely. Regulatory systems and protein-protein associations and protein-protein interactions remain unresolved. In addition, the method still has a bias against membrane-bound proteins. Lastly, information on PTMs is lost, unless directed approaches are applied to identify and quantify these protein modifications (see Section 4.2). Despite these shortcomings, shotgun proteomics provides us with a most powerful tool in 
hypothesis-generating research or to answer questions by well-designed and well-focused experiments.

\subsection{Membrane-bound systems}

\subsubsection{Analysis of membrane-bound proteins}

Above, we noticed that membrane-bound proteins are recovered only poorly by 2DE-MS, whereas they remained underrepresented in shotgun proteins. In their review on early status of proteomics, Santoni et al. (2000) even wondered if proteomics and membrane proteins would not be "un amour impossible". In the years that followed, lots of efforts and ingenuity have been invested to enhance the detection and identification of membrane proteins (see for reviews: Bunai and Yamane, 2005; Braun et al.., 2007; Lu et al., 2008; Poetsch and Wolters, 2008; Rabilloud et al., 2008a,b; Weiner and Li, 2008; Bernsel and Daley, 2009; Gilmore and Washburn, 2010; Helbig et al., 2010; Savas et al., 2011; Vuckovic et al., 2013; Soufi and Macek, 2015). Reviewing the outcome of these pursuits nine years later, Rabilloud (2009) was less pessimistic: "love is possible, but so difficult".

The reasons why proteomic analysis of membrane proteins is often -but not alwaysdifficult may become clear if we look at the nature of these proteins, thereby defining what is implied by a membrane protein, or more specifically a cytoplasmic membrane protein (Fig. 10) $<$ Fig. 10 $>$. These proteins come in a number of classes with different topologies. Simple ones cross the membrane by one trans-membrane helix (TMH), their $\mathrm{N}$ - and C-terminal parts being localised on opposite sides of the membrane. Terminal parts are comprised of hydrophilic peptide chains of which lengths may vary substantially. More complex membrane proteins have multiple TMHs. One such TMH is composed of a stretch of 15-25 mostly hydrophobic amino acids that fold into an alpha helix. The hydrophobic helices make the protein to reside inside the membrane bilayer. In this bilayer, proteins are packed by annular 
lipids that act non-specifically as "lubricant" and "solvent" to allow rotational or lateral motions of the protein, or by non-annular lipids, like cardiolipin (see Section 4.1.4) that may be determinants in the structure and activity of the membrane protein (Lee, 2003; Paradies et al., 2014). By the presence of both hydrophilic and hydrophobic regions, membrane proteins are amphipathic to variable degrees. This amphipathic character can be expressed by the grand average of hydropathicity (GRAVY) index (Kyte and Doolittle, 1982). In general, the more THMs are present, the more hydrophobic the protein becomes and the more difficult the protein is detected by proteomic analyses. Proteins may also be just anchored to the membrane by hydrophobic and/ or ionic interactions (Fig. 10). Alternatively, contact is facilitated by lipids or by glycosylphosphaditylinisositol (GPI) moieties that are covalently bound to the protein. These proteins lack any $\mathrm{TMH}$, which may hamper their immediate identification as membrane proteins by genomic analyses. In case of multi-subunit respiratory enzymes, complexes can be composed from different types of membrane proteins depicted in Figure 10. Here, association may also result from subunit-subunit rather than from proteinlipid interactions.

The main problem in proteomic analysis of membrane proteins is how to cope with hydrophobicity. In the absence of lipids, membrane proteins readily precipitate or aggregate in an aqueous environment having a $\mathrm{pH}$ close to the $\mathrm{pI}$ of the proteins (Wu et al., 2003; Eichacker et al., 2004). This is less of a problem for proteins with low GRAVY indices $(<0.1-$ 0.2), i.e. proteins that harbour only one or two THMs and/or contain hydrophilic regions of sufficient length. Generally, such proteins can be identified by conventional 2DE or shotgun approaches. Membrane lipids may be replaced by ionic detergents that pack the protein in a micelle, making the protein soluble in water. SDS has superior qualities in this respect and, in fact, SDS-PAGE is most suitable for the separation and analysis of membrane proteins. However, the presence of SDS is incompatible with IEF. A second problem regarding the 
proteomic analysis of membrane proteins is that TMHs lack sufficient (hydrophilic) lysine and arginine residues for proteolytic cleavage by trypsin, yielding peptide fragments that are out of range for conventional peptide-centric MS analysis (Helbig et al., 2010). Still, appropriate trypsin cleavage sites may be present in the hydrophilic domains, permitting the identification of the protein. Thirdly, membrane proteins may be present in too low amounts or are masked by highly abundant proteins. Obviously, low abundance may not apply to respiratory membrane proteins related with the primary energy metabolism. In this case, the questions would be, which proteins are measured detected and which ones are not and why these proteins were not detected. The listed problems have been be tackled at two levels: the development (1) of procedures to enrich membrane proteins or proteins of interest and (2) of methods that enhance their detection and identification by MS.

Proteins residing in the membrane can be enriched by differential (ultra)centrifugation steps, sucrose gradient ultracentrifugation or gel filtration column chromatography. Taking advantage of membrane hydrophobicity, a biphasic partitioning system might be advantageous in separating the hydrophobic (membrane) from the hydrophilic (cytoplasmic) protein complement (Everberg et al., 2006, 2008; Poetsch and Wolters, 2008; Masuda et al., 2009). In this procedure, the hydrophobic fraction is extracted into the more hydrophobic polyethylene glycol (PEG) phase, while hydrophilic proteins partition into the hydrophilic dextran phase. The applicability of this method in prokaryotic research does not seem to be tested as yet. Besides, the presence of PEG is extremely incompatible with MS and the compound has to be carefully removed before LC-MS/MS or MALDI-MS. Another tool for the enrichment of the membrane subcellular fraction, which has not been exploited in this research, is free flow electrophoresis (Zischka et al., 2006; Eichacker et al., 2015). However, highly abundant cytoplasmic proteins can still contaminate membrane-bound protein fractions. These contaminants may be removed by incubation in alkaline solvent systems, for 
instance a high concentration of carbonate (Fujiki et al., 1982; Molloy, 2008) or of $\mathrm{NaBr}$ (Schluesener et al., 2005). Under alkaline conditions, membranes spread as sheets (Fujiki et al., 1982; Wu et al., 2003) and non-specifically associated proteins are dissociated, perhaps including ones of which membrane association is functional. Further enrichment may involve hydrophilic interaction chromatography (Hendrickx et al., 2015). After tagging peripheral lysines with biotine, proteins present in membrane fractions can be further enriched by the using of avidine/streptavidin beads or columns (Rybak et al., 2004; Scheurer et al., 2005; Schiapparelli et al., 2014). As far as relevant in bacterial research, lectin affinity column chromatography could provide a tool to selectively enrich glycosylated membrane proteins (Zhou et al., 2007; McDonald et al., 2009). If investigations address one or a small subset of membrane proteins and their binding partners, antibody affinity chromatography can also be a method of choice (Section 4.1.1) (Banks et al., 2012).

Procedures to enhance the MS-based detection of membrane proteins developed along the two lines described before: 2DE-MS (Bunai and Yamane, 2005; Braun et al., 2007; Kashino et al., 2007) and shotgun proteomics (Lu et al., 2008; Rabilloud, 2009; Vuckovic et al., 2013). Regarding 2DE, the issue is to dissolve the proteins from the membrane and transfer them without precipitation to a detergent mimicking the membrane environment, so that gel electrophoresis becomes feasible. Dissolution with 1\% SDS, an anionic detergent, provides these opportunities, but membrane protein complexes will dissociate and the possibility of a second dimension will be excluded: Upon SDS-PAGE in the second dimension, proteins are just distributed across the gel along a diagonal, which is not very informative. To an extent, off-diagonal resolution can be improved if separations in the first and second dimensions are carried out under different conditions with respect to PAGE (gradient) concentrations and the composition of the gel electrophoresis buffer systems (Braun et al., 2007). An alternative for SDS has been found in the cationic detergents 16- 
benzyldimethyl-n-hexadecylammonium chloride (16-BAC) (Zahedi et al., 2005, 2007; Bisle et al., 2006; Nothwang and Schindler, 2009) and cetyltrimethyl-ammonium bromide (CTAB) (Helling et al., 2006). In these methods, membrane proteins are dissolved by treatment with a mild detergent and packed by a layer of 16-BAC or CTAB molecules, which gives charge for PAGE separation in a discontinuous acidic gradient. As for SDS-PAGE, separation proceeds on the basis of the size of the proteins. Hence, after 16-BAC and SDS electrophoreses in the first dimension and second dimensions, respectively, proteins are again are resolved along a diagonal. Future improvements have to be directed towards getting more evenly distributed protein patterns across the whole 2D gel. Two such, very robust method are Blue Native (BNE) and Clear (or Colourless) Native (CNE) gel electrophoresis, which will be described hereafter (Section 3.2.2). While detergents are essential to resolve proteins from the membrane and their presence is needed during gel electrophoresis, these compounds may hamper MS analysis severely, an aspect that also holds for shotgun proteomics. Consequently, detergents have to be carefully removed before those analyses. Otherwise, this issue may be overcome by the application of laser-induced liquid bead ion-desorption (LILBID)-MS (Sokolova et al., 2010).

Shotgun-proteomics-supported methods aimed at the improved identification of membrane proteins have been focused on protein cleavage procedures (Helbig et al., 2010). Trypsic digestion of intact membrane proteins usually yields only the subset of peptides that are present in the hydrophilic domains (“membrane shaving”). Although this information is important for determining the topology of a membrane protein (see next), it may be insufficient for an unambiguous identification, creating the need to access the complete protein sequence embedded in the membrane. Again, SDS would be a perfect agent to dissolve membrane proteins, but this compound compromises protease activity. The presence of $50 \%$ methanol dissolves the membranes, but keeps the proteins intact, enabling the 
targeting of intra-membrane cleavage sides. As the hydrophobic parts of membrane proteins contain only few cleavage sites for trypsin, various methods have been tested in order to obtain a more comprehensive peptide coverage, including combined chemical (CNBr) and proteolytic cleavages by trypsin or other proteases (Fischer et al., 2006; Fischer and Poetsch, 2006; Poetsch and Wolters, 2008). One of these proteases is Proteinase K (ProtK) (Wu et al., 2003). This protease cleaves a protein ultimately in dipeptides, which are irrelevant for MS analyses. However, if carried out under alkaline conditions, ProtK activity is strongly inhibited, yielding peptide fragments that are sufficiently sized for those analyses.

We just indicated that membrane shaving might give important topological, structural information, indicating on which side of the membrane the identified peptides occur. For instance, when performed on protoplasts only those peptides that face the periplasm will be recovered. In addition, the absence of peptides in the $\mathrm{N}$ - or C-terminal part of the protein may hint at proteolytic cleavage related with protein export and protein assemblage.

As mentioned in the beginning of this section, many efforts have been invested in elucidating membrane proteomes, including those of a variety of prokaryotic species. Table 2 presents a selected overview of investigated species and of the methods employed. <Table 2>. Results were satisfactory or even very promising, especially if investigations had been performed by complementary approaches. In retrospect (2015), one could even state that membrane proteins and proteomics are becoming to like each other at second sight, but it is somewhat early to speak of love.

\subsubsection{Membrane-bound protein complexes}

Respiratory RMPs are usually composed of several subunits. In the global approaches based on 2D IEF SDS-PAGE and shotgun MS the information regarding subunit composition is lost. In the first method, RMPs are resolved as separate polypeptides, whereas in the second 
method peptide fragments from enzyme complexes are immersed in an ocean of other peptides, which are only allocated to single subunits. Most elegant and powerful methods that overcome these restrictions are BNE (Schägger et al., 1988; Schägger and Von Jagow, 1991) and the related CNE (Wittig and Braun, 2006; Wittig and Schägger, 2008a, 2009a; Wittig et al., 2007). In BNE, membrane proteins are resolved from the membranes by mild neutral detergents, like Triton X-100, dodecyl- $\beta$-D-maltoside (DDM) or digitonin, and in the presence of 6-aminohexanoic acid, a zwitterionic compounds that improves membrane solubilisation. Hereafter, anionic dye Coomassie Brilliant Blue G-250 (CBB) is added. The critical step is to find the conditions at which subunit interactions and higher-order protein interactions (supercomplexes) are preserved. Extracted protein complexes get embedded in a layer of CBB molecules. The dye binds hydrophobic proteins and gives them a negative charge allowing their solution in aqueous systems and their separation by gel electrophoresis. Because the dye also binds cationic amino acids of soluble proteins, BNE is also suitable for this type of proteins. After extraction, the dissolved protein complexes are separated by PAGE using a designated buffer system containing CBB. Separation occurs on the basis of protein sizes. By varying the concentrations of polyacrylamide or by applying different gradients, protein complexes with molecular masses as low $10 \mathrm{kDa}$ and as high as $10 \mathrm{MDa}$ are resolved as blue bands (Wittig and Schägger, 2008a, 2009a; Strecker et al., 2010; Wittig et al., 2010). Upon separation in the second dimension by SDS-PAGE, subunits of protein complexes or the constituents of supercomplexes are diverted into their individual polypeptides that in turn can be identified by MS. Importantly, polypeptides derived from a single protein complex migrate below to each other at the same width of the SDS gel, which facilitates their immediate allocation to a certain protein (super)complex. CNE works in principal the same, except that CBB is omitted from the electrophoresis buffer (Wittig et al., 2007). In this method, protein complexes (first dimension) or individual subunits (second dimension) are 
colourless and can be stained by fluorescent dyes. Such staining is not possible in case of BNE since CBB quenches fluorescence. Due to the absence of CBB in the buffer system of $\mathrm{CNE}$, this method is restricted to acidic proteins and their intrinsic migration through the electric field. As a result, protein resolution in these gels is far less than for BNE, and proteins are often observed as smears rather than as defined bands (Wittig and Schägger, 2005). This problem has been addressed by including colourless mixed micelles of neutral and anionic detergents to the gel electrophoresis buffer ("high-resolution CNE", hrCNE; (Wittig et al., 2007)).

A rapidly increasing number of studies on bacterial ETS has benefitted from the method of BNE (Table 2); our work on M. thermautotrophicus was one of the first (Farhoud et al., 2005). Here we investigated the membrane-bound and soluble protein complexes of this organism grown under high and low hydrogen concentrations. Fig. $11<$ Fig. 11> presents an example of one of the two-dimensional BN gels obtained. Protein spots were analysed by MALDI-TOF MS and LC-MS/MS. Although only part (1550) of these protein spots was investigated, 361 unique proteins could be identified, including all key enzymes involved in methanogenesis, the soluble and membrane-bound ones alike. Also $\mathrm{H}^{+}$-translocating $\mathrm{F}_{1} \mathrm{~F}_{\mathrm{O}^{-}}$ ATP synthase was recovered. This enzyme was found as monomeric and dimeric complexes, and as higher order aggregates (Figs. 11, B-C), which never has been shown for prokaryotes, but has firmly been established for mitochondrial ATP synthase (see Section 5.1.1) (Wittig and Schägger, 2008b, 2009b). Similarly, the multi-subunit membrane-bound $\mathrm{Na}^{+}$-dependent $\mathrm{N}^{5}$-methyltetrahydro-methanopterin:coenzymeM methyltransferase (MTR) occurred as monomeric and dimeric complexes, and part of it was even associated with ATP synthase (Fig. 11C). Importantly, association was not an experimental artefact, but it was related to the growth conditions applied. Besides this, the change in growth conditions revealed that soluble benzyl viologen reducing hydrogenase and soluble heterodisulfide reductase differentially 
formed complexes (Fig. 11D). This observation could be of relevance in the further understanding of how the exergonic terminal step of methane formation, methylcoenzyme $\mathrm{M}$ reduction, is coupled to the endergonic first step of $\mathrm{CO}_{2}$ reduction under hydrogen limitation by an electron bifurcation mechanism (Thauer et al., 2008; Kaster et al., 2011).

Recently, we used the new BNE-based method of complexome profiling to make an inventory of the enzymes and respiratory complexes that play a role in the energy metabolism and cell carbon synthesis of anammox bacteria (our unpublished results). The particular method was pioneered by Wessels et al. (2009) to identify new assembly factors of mitochondrial complex I and was later on optimised (Remmerie et al., 2011; Heide et al., 2012; Weber et al., 2013; Huynen et al., 2015). In this method, proteins and their complexes isolated from the membrane fraction are separated on a BN gel as described above. Instead of the separation in the second dimension by SDS-PAGE, the gel is cut in multiple pieces each of which are proteolytically cleaved and subjected to label-free quantitative LC-MS/MS. Such separation is shown in Figure $12<$ Fig. 12> for a membrane preparation of the anammox bacterium Kuenenia stuttgartiensis. LC-MS/MS not only identifies the proteins that are found in each gel slice, but also quantifies their relative amounts on basis of the intensity of the mass spectral peaks (Section 2.3.2). The distribution if a certain protein across the whole gel can then be deduced based on the protein migration patterns. Finally, these protein profiles are hierarchically clustered, eliciting which proteins co-migrate as protein complexes through the BN gel. The application of various polyacrylamide gradients thereby assures reproducibility of the migration profiles. After analysis of the K. stuttgartiensis membrane preparations, nearly 1400 different proteins could be identified, representing 34.5\% of the transcribed genome. $44.4 \%$ of the proteins with one predicted TMH were recovered. This value might be an underestimation, since a single TMH could be a signal sequence that is cleaved during protein translocation, making the protein a soluble one in situ. Proteins with two or more 
predicted TMHs were recovered with $>50 \%$ efficiency, irrespective of the number of TMHs (2-15). Most importantly, most if not all of the subunits of NADH:quinone dehydrogenase (complex I), membrane-bound formate dehydrogenase, three different types of $b c_{1}$ complexes and four different types of ATP synthase/ ATPase, and various other respiratory RMPs known from other species were retrieved. Subunits were consistently assembled into protein complexes as predicted from genome analyses (Kartal et al., 2013). In addition, the presence of a number of predicted, new anammox-specific RMPs could be verified as well.

Results obtained with BNE in the research on eukaryotic and prokaryotic ETS (Table 2), and as illustrated by the above two examples, underscores the broad and novel potentials of this technique. This method appears to be particular useful in studies on the composition and assembly of RMPs (Section 5). Presently, BNE has been given one more dimension by the introduction of metal isotope native radio autography in gel electrophoresis (MIRAGE) (Sevcenco et al., 2012). In this application, cells are grown in the presence of radioactive isotopes of metals (Fe, $\mathrm{Zn}, \mathrm{Cu}, \mathrm{Mo}, \mathrm{W}$ ) that play a key role in respiratory complexes. The subsequent analysis of these cells by BNE in combination with radio autography establishes which metal is incorporated into which protein. As an alternative method to map the “metalloproteome" of an organism without the use of radioactive metals, pre-fractionation of whole-cell lysates by conventional column chromatography has been suggested (Cvetkovic et al., 2010; Maret, 2010; Lancaster et al., 2011; Shi and Chance, 2011). Colum fractions are subjected to ICP-MS (inductively coupled plasma mass spectrometry) to detect the presence of metals and relevant fractions are further analysed by BNE combined with tandem LCMS/MS. Instead of conventional column chromatography, immobilised-metal affinity chromatography may serve the enrichment of specific metalloproteins from complex samples (Sun et al., 2008; Block et al., 2009; Shi et al., 2011; Cheung et al., 2012). 


\subsection{Differential expression}

The bacterial cell is a highly dynamic system that has to be optimally tuned for changes in its environment, such as the supply in its energy sources. This tuning relies on the adaptation of the enzymatic machinery, both by the differential expression at the transcriptional level and, more directly and elegantly, by PTMs of proteins to control their activities. Proteomics might provide the toolbox to investigate these adaptive processes on a global scale and at both levels simultaneously while taking into account the limitations of global approaches (see Section 3.1). Hereafter we will outline the proteomics-based methods and applications to map the regulation of bacterial ETSs, following the two routes along which proteomics has evolved, 2DE and shotgun proteomics. Regulation at the post-translational level will be described separately (Section 4.2).

The strength of 2DE is its capacity to resolve and visualise a vast amount of proteins on a 2D gel. Already in the pre-proteomics era, these merits were appreciated. By changing growth conditions or implementing a stress situation together with the addition of ${ }^{35} \mathrm{~S}$ - or ${ }^{14} \mathrm{C}$ labeled substrates, the proteins that were synthesised in response to the particular change could be revealed by autoradiography (see f.i. VanBogelen et al., 1996). At that time, the challenge was to identify these proteins. The introduction of MS has solved the issue. The close inspection of two or more 2D gels of lysates from cells grown under varied conditions immediately demonstrates changes in expression patterns. Fig. 9B shows a simple, but puzzling example of this. $M$. thermautotrophicus contains one protein with a biotin prosthetic group, the beta subunit of pyruvate carboxylase (PycB, MTH1107). This enzyme introduces fixed $\mathrm{CO}_{2}$ into the TCA cycle for further distribution across anabolic pathways. $M$. thermautotrophicus perfectly grows without biotin administration, but the organism lacks the pathway for its biosynthesis (J. Keltjens, unpublished results). In agreement herewith, MTH1107 found on 2D gels (Fig. 9B) is not biotinylated. Strikingly, after growth in the 
presence of this cofactor the MT1107 spot is moved to another position and here the protein harbours covalently bound biotin. The puzzling aspects are, why pyruvate carboxylase is expressed in an inactive form and how fixed $\mathrm{CO}_{2}$ is fed into the TCA cycle.

The close comparison of highly complicated protein distribution patterns among different gels is facilitated by gel imaging and special software programmes. By measuring the sizes and intensities of the stained spots ("spot volumes"), differences in protein expression levels can be evaluated quantitatively. The problem, however, is that proteins never migrate in exactly the same way due to minute differences in sample and gel preparation and electrophoresis conditions. In principle, this problem can be computationally addressed by the application of programmes that resize and reshape gel images, so that a set of marker proteins overlay each other (“warping”), but this warping is not without faults, resulting in a substantial rate of false-positives or false-negatives. Difference gel electrophoresis (DIGE) addresses this problem (Viswanathan et al., 2006; Larbi and Jefferies, 2009; Sapra, 20091 Minden, 2012). In this method, protein samples prepared from two differently grown cell cultures are labelled with two different fluorescent markers. Equal amounts of proteins from both samples are mixed and labelled with a third marker to serve as an internal control. Before 2DE, these three samples are combined and separation is performed in one gel. Subsequent gel imaging that exploits the high dynamic range (4 log) of the fluorescent markers reveals which proteins are differentially expressed and to what degree. Figure $13<$ Fig. 13> shows an example from our own work on $M$. thermautotrophicus (Farhoud, 2011). While the translated genome predicted the presence of 877 different proteins in the $\mathrm{pH}$ 3.5-5.5 range covered by the IEF strip, more than 2500 fluorescent protein spots could be detected in mixed preparations from cells grown with excess and limiting hydrogen concentrations. Eight of these were differentially expressed more than 5-fold, 18 of these 3 to 5-fold, 69 by a factor 2-3, and 214 by a significant 1.15 to 2-fold. Importantly, differential 
expression comprised of a number of protein proteoforms ("trains"). This concerned, among others, MTH1872 (Fig. 13), a homolog of the eukaryotic Translation Initiation Factor $2 \alpha$ (aIF2 $\alpha$ ). In Eukarya and Archaea (Tahara et al., 2004, Schmitt et al., 2009; Andaya et al., 2014), aIF2 $\alpha$ activity is known to be controlled by phosphorylation. This could be the case for M. thermautotrophicus, but a dedicated MS method would be needed to substantiate this prediction.

Shotgun proteomics is a convenient tool for the global inventory of expressed proteins (Section 3.1.2), but the approach is even well suited to determine and quantify differences in protein expression levels. The prerequisite for its application is the availability of highly reproducible protocols for the preparation of samples obtained from whole-cell lysates or subcellular fractions of interest. For differential expression quantification, protein samples of cells cultured under the varied conditions are labelled in vitro or in vivo as explained in Section 2.3.2. Like in the DIGE methodology, labelled and unlabelled preparations are mixed before 1DGE, LC or MudPIT separations and subsequent multiplexed tandem MS, in order to avoid analytical artefacts and to facilitate mass spectral comparisons. As before (Section 3.1.2), a drawback in shotgun MS protein identification and quantification is that PTMs may go unnoticed. Interference of PTMs with peptide ionisation and the complete loss of information regarding protein proteoforms may even compromise the correct interpretation of data.

Hitherto, a large number of studies took advantage of 2DE- and/ or shotgun-based proteomics to map differential expression patterns of bacteria in response to a change in environmental conditions. Table $3<$ Table $3>$ presents a selected overview of studies that focused on the effect of changes in the supply of primary electron donors or terminal electron acceptors. In general, expression profiles are complex and involve numerous proteins. Besides this complexity, it should be taken into account that views may be biased because of flaws in 
protein detection, such as membrane proteins. Nevertheless, in concert with genomics, transcriptomics and metabolomics, proteomic analyses may contribute significantly to systems biology endeavours. This is especially true if protein expression levels are profiled on the basis of series of experiments performed under carefully controlled conditions (see f.i. Wegener et al., 2010; Kohlmann et al., 2011, 2014; Soufi et al., 2015). Moreover, findings can be completely unexpected, thereby raising hypotheses for further, more directed investigations aimed at the elucidation of underlying regulatory systems. The role of proteomics in this, however, may be restricted, since regulatory proteins that exert their function in modified forms are usually present in low copy numbers, precluding their detection by MS-based methods. If approached in a targeted way (Section 2.3.3), proteomics could provide a most valuable complementary tool. The comparison of expression patterns between mutant and wild type strains immediately shows the effect of a mutation (see f.i. Section 5.2.2). Herewith, known observations are substantiated by another technique, but the comparison also may disclose new proteins whose expression is affected by particular mutation, which opens directions for further research.

\section{RESPIRATORY PROTEIN COMPLEXES IN ASSEMBLY}

RMPs represent a notoriously difficult class of proteins to work with within the field of structural biology. Usually, these proteins are fairly resistant towards crystallisation, and a load of trial-and-errors (and tricks) is required to get crystals of sufficient refractory quality that yield high-resolution X-ray images (Moraes et al., 2013; Loll et al., 2014). The other method to explore protein structures, NMR spectroscopy, does not depend on protein crystals, but this approach is still limited in its scope regarding the size of the proteins. Even though Xray crystallography and NMR give the most detailed structural information, this information may not necessarily reflect a physiological state. Crystallisation trials and NMR studies need 
a vast amount of purified protein by often elaborate column chromatographic methods. During purification, essential components could be lost. In addition, purified proteins are investigated in a non-natural environment, which is the cell lipid membrane. Again, lipids that play a key role in structural integrity may get lost and may be replaced by artificial detergents. To speed up large-scale purification, enzymes are often (heterologously) overexpressed in tagged forms, but overexpression represents a non-physiological condition. Furthermore, heterologously overexpressed proteins may be devoid of PTMs that could be structurally or functionally important. Lastly, crystal structures and NMR spectra present only a static view. Enzyme action is highly dynamic and it involves a multiplicity of conformational states, substrate-protein and protein-protein interactions.

In recent years, we witnessed a boom of mass spectroscopic methods and techniques aimed at the resolution of the structural properties of RMPs and dynamic processes related with their catalytic action, including post-translational modifications. The common property of these methods and techniques is that they take the whole protein as the starting point, ushering the fields of top-down and structural proteomics. For quite some time, the development and application of these expensive and often home-built apparatus has been the playground of specialists. Regarding the study of electron transfer processes, these specialists took as their "toys" mitochondrial OXPHOS complexes from human tissues or, to simplify matters, from animal or yeast model organisms. The focus on these OXPHOS complexes is understandable, considering the manifold of metabolic diseases that are caused by the malfunctioning of mitochondrial respiration. All in all, application of these new methods to advance our knowledge on bacterial ETSs and their regulation has lagged behind. Hereafter, we will present an overview of top-down proteomics that allowed, or may allow, the better comprehension of the structure of bacterial respiratory RMPs and their regulation at the level of PTMs. We will exemplify progress in this knowledge mainly with achievements in 
research on eukaryotic systems, which just reflects the current status of the field, and on prokaryotic systems, as much as available. The examples of the work on OXPHOS complexes permit an illustration of the promises that new proteomics-based methods offer, but also of their limitations, as to invite their critical implementation in the investigations on bacterial systems. For the interested reader who likes to learn more about new developments and their applications, we would like to refer to a number of recent reviews (Zhou and Robinson, 2010; Cui et al., 2011; Owens, 2011; Hyung and Ruotolo, 2012; Catherman et al., 2014; Petrotchenko et al., 2014; Boersema et al., 2015; Marcoux and Cianférani, 2015).

\subsection{Structural aspects of respiratory complexes}

\subsubsection{Protein purification}

An enormous advantage in the study of RMPs by MS techniques is that analyses need only minor amounts of protein (less than a microgram). Nevertheless, preparations need to be as pure as possible and protein complexes should be catalytically active, implying that they should be composed of all subunits in the correct stoichiometry at which the overall structure is maintained as found in the original membranous milieu. The latter may comprise the presence in the right quantities of native structural lipids. Obviously, purification can be achieved by conventional means. Because only minute protein amounts are required, efforts have been, and are being directed at scaling down the repertoire of column chromatographic methods and of new separation methodologies to the nanoliter volumes, such as nanoparticlebased monoliths (Catherman et al., 2014; Tang et al., 2014). Otherwise, purification by BNE and elution from the gels might provide a sufficient amount of pure protein for MS analyses.

An approach that has been especially designed for proteomics is affinity purification combined with MS (AP-MS), or variants of it like FLAG-tag (Terpe, 2003) and tandem affinity purification (TAP-MS) (Rigaut et al., 1999; Puig et al., 2001; Vogel et al., 2005; 
Saada et al., 2009). In the original TAP-MS procedure, the target protein is fused in frame with two tags separated by a TEV (tobacco etch virus) cleavage site and expressed. The proximal tag is a calmodulin-binding peptide and the second one is composed of the IgGbinding part of the Staphylococcus aureus protein A. This setup allows a two-step purification of the target protein and its binding partners, but the method has its faults. The size of protein A (approximately $21 \mathrm{kDa}$ ) may affect the folding, activity of the protein (complex) or interaction with the physiological binding partners. Purification is rather harsh and these partners may get lost. Moreover, despite the stringent conditions, the protein (complex) of interest may not be pure. Sometimes, up to 95\% of all proteins that co-purify are contaminants and their presence requires a tedious investment in MS and data analyses time to identify the right candidate(s) (Meyer and Selbach, 2015). In addition, data may be ambiguous in establishing which protein belongs to what complex. A reason for the high detection rate of contaminants is the extreme sensitivity of modern MS apparatus. To address these issues, several variants of the original method have been proposed, including the use of smaller tags (Gingras et al., 2007; Völkel et al., 2010; Trinkle-Mulcahy, 2012). Another solution is quantitative affinity purification (q-AP-MS) (Vermeulen et al., 2008; Meyer and Selbach, 2015). Herein, differentially SILAC-labelled cells are transfected with the tagged protein to be investigated, whereas a control vector contains the tag only. After immunoprecipitation, elution, protein digestion and shotgun proteins, lowly abundant contaminant proteins are always recovered in a 1:1 ratio and can be ignored. On the basis of the relative changes in peptide intensities, the subunit composition of the protein complex to be characterised is derived.

\subsubsection{Primary structure information}

Once purified, the identity of the subunits and subunit stoichiometry of a protein complex can be analysed. Subunits can be identified in the traditional way using SDS gel electrophoresis or 
2D-BNE and subsequent peptide mass fingerprinting or shotgun MS at which the absence of specific peptides is indicative of $\mathrm{N}$ - or C-terminal cleavage events. Top-down proteomics explores the whole protein or protein complex inside the mass spectrometer. Here, the sample is volatised under atmospheric pressure and by soft ionisation (Fig. 14) < Fig. 14>. Conditions are chosen such that subunit interactions and other non-covalent bindings are preserved. Herewith, PTMs remain intact and are subjected to further identification (see Section 4.2). After increase of ion activation (collision energy), the complex disintegrates into its constituent subunits of which the molecular masses can be determined, simultaneously enabling the establishment of the subunit stoichiometry (Hopper and Robinson, 2014). Further increase of the ion activation results in the fragmentation of the polypeptide chains, which can be fully sequenced. Interestingly, first fragments observed stem from membrane-embedded regions. Hence, by the deliberate tuning of ionisation energies, the relation between higherorder structure and protein sequence can be probed in a single experiment (Konijnenberg et al., 2015).

Precise native MW measurements pose high demands on the resolution of the mass spectrometer, since it would have to distinguish between S-H groups and disulphide bridges $(\Delta m=2 \mathrm{Da})$, deamidation $(\Delta m=1 \mathrm{Da})$, acetylation versus trimethylation $(\Delta m=0.04 \mathrm{Da}, \mathrm{sic})$ and phosphorylation versus sulfation $(\Delta m=10 \mathrm{Da})$. An inherent problem of molecular mass measurements is that the parent ion [M] is distributed across multiple peaks as the result of natural ${ }^{1,2} \mathrm{H}$ and ${ }^{12,13} \mathrm{C}$ isotope ratios (Section 2.1.1; Figs. 2, 7 and 8).

Quite a few studies on respiratory systems have been supported by MS-based methods to obtain information on the subunit composition and subunit primary sequences. As alluded to above, most of these were related to mitochondrial OXPHOS complexes. Studies were directed either at the full respiratory repertory of mitochondria from humans (Wessels et al., 2009, 2013; Catherman et al., 2013), plants (Klodmann et al., 2011) and yeasts (Lemaire and 
Dujardin, 2008; Helbig et al., 2009; Nübel et al., 2009), or specifically at eukaryotic complex I (Carroll et al., 2003; Pocsfalvi et al., 2006; Fearnley et al., 2007; Batista et al., 2010; Bridges et al., 2010; Klodmann et al., 2010; Dröse et al., 2011; Klodmann and Braun, 2011), complex III (Marín-Buera et al., 2015), or ATP synthase (Wittig and Schägger, 2008b; Hoffmann et al., 2010). Hitherto, only few investigations have explored bacterial respiratory systems from the proteomics perspective at some depth (Battchikova and Aro, 2007; Menon et al., 2009; Castelle et al., 2015), albeit with most promising results regarding SC formation. The latter work will be discussed in Section 5.1.

\subsubsection{On track to secondary, tertiary and quaternary structures of respiratory complexes}

Proteomics does not only provide invaluable data on protein sequences, but nowadays approaches also allow an insight also in the three dimensional organisation of a protein complex, lending the field a firm place within structural biology (Benesch and Robinson, 2006; Benesch et al., 2006; Zhou and Robinson, 2010; Hyung and Ruotolo BT, 2012; Petrotchenko and Borchers, 2014). To this end, a number of tools and methods have been established that in combination permit the proposal of structural models, even though of asyet low resolution (Konermann et al., 2008; Owens, 2011). Most methods are still peptidecentric and are directed at the immediately accessible part of a protein, its surface. These methods are schematically represented in Figure $15<$ Fig. 15 $>$.

A method that actually has been used for decades to probe conformational features of proteins is limited chemical or enzymatic proteolysis (Fontana et al., 2004, 2012; Feng et al., 2014). We already referred to it in the context of "membrane shaving" (Section 3.2.1). The first peptides that are released by this treatment are the ones exposed to the (aqueous) environment and their identification provides information on the topology of an RMP. If applied under different physiological conditions, limited proteolysis also may inform on 
conformational changes and the dynamics of an enzyme. Amino acids and peptides are susceptible to chemical modifications. This property is utilised by the treatment of whole proteins with reagents that bring about known modifications (see for a review: Bennett et al., 2000). Again, proteolysis and subsequent tandem MS discloses those parts of the enzyme complex that have been modified (or have resisted modification). "Protein painting” is a recent further development of the approach in which small chemical dyes are used to probe the protein (Luchini et al., 2014). Alternatively, surface-exposed amino acids are oxidatively modified by exposure to hydroxyl radicals, once more permitting the elucidation of targeted peptides (Konermann and Pan, 2012). Whereas chemical modifications address specific amino acids, hydrogen/deuterium exchange coupled with MS (HDX-MS) exploits the property that all amino acids (except for proline) can exchange amide hydrogen atoms for deuterium, which permits more homogeneous protein labelling (Mandell et al., 2005a, 2005b). When incubated in a $\mathrm{D}_{2} \mathrm{O}$-containing buffer, the $\mathrm{H} / \mathrm{D}$ exchange rates are not the same for all amides. Rates will generally be highest for the $\mathrm{D}_{2} \mathrm{O}$-exposed parts of a protein. This difference in rates is advantageous in identifying these parts or to investigate kinetics of a protein system (Tsutsui et al., 2007). HDX-MS has been widely used in bottom-up proteomics (see for reviews: Iacob and Engen, 2012; Marcoux and Cianférani, 2015; Boersema et al., 2015). After incubation in the $\mathrm{D}_{2} \mathrm{O}$ buffer and quenching the exchange reaction at low pH 2.4 and $0-4^{0} \mathrm{C}$, followed by pepsin cleavage, tryptic fragments are analysed by LC-MS/MS as usual. This method, however, also has its flaws. Peptide fragments may go unnoticed or unidentified, spectra are extremely complicated and LC separation may cause H/D scrambling and back reactions. Nevertheless, HDX-MS is fully compatible with a topdown approach where these drawbacks can be overcome (Bache et al., 2008; Konermann et al., 2011; Rand et al., 2014; Burns et al., 2015). 
Another chemical modification procedure that provides structural information is chemical crosslinking (CX-MS) introduced by Rappsilber et al. (2000) and Young et al. (2000), and recently reviewed in (Petrotchenko, 2010; Rappsilber, 2011; Leitner et al., 2010, 2014; Ramisetty and Washburn, 2011; Sinz et al., 2015; Tran et al., 2015). In this method, two amino acids are connected to each other through a reagent with two (or more) reactive side groups. Nowadays, a range of crosslinkers is available like N-hydroxysuccinimide derivatives that covalently bind to the $\mathrm{N}$-terminus and the $\varepsilon$ amine group of lysine residues, sulfhydryl reactive crosslinkers, crosslinkers directed at the C-terminus and the carboxylic group of aspartates and glutamates, and photo-reactive crosslinkers. Their main difference is the spacing between the reactive groups (5-15 $\AA$ ). Since these lengths are known, the reagents act as rulers measuring the maximal distance between the targeted amino acids. Application of CX-MX, however, has its challenges. The number of suitable crosslink sites is limited or too many (in large proteins). MS spectra are quite convoluted and additional purification steps are required to recover large polypeptides that have evaded proteolytic cleavage for further investigation. These issues have been solved to an extent by cleavable linkers. In addition, software programmes have been developed that can handle cross-linked peptides (see: Tran et al., 2015).

The simplest crosslinker is formaldehyde that is water-soluble, permeates the cell membrane and forms reversible short-linked bonds (2.3-2.7 $\AA$ ) (Ramisetty and Washburn, 2011). Despite recent promising results, the use of formaldehyde still needs thorough testing: this compound not only acts as a crosslinking agent but it is capable of various other complicating chemical modifications as well (Srinivasa et al., 2015).

Most studies that employed above methods were focused on soluble proteins. Research on respiratory RMPs is still in its infancy (Zhou and Robinson, 2014). The reason may be clear: RMPs are cumbersome to handle and difficult to analyse by MS (see Section 
3.2.1). In addition, membrane-embedded, hydrophobic regions may be inaccessible for reagents used for peptide modification. For instance, amino acids required for crosslinking are rather rare in hydrophobic domains. Yet, crosslinking may stabilize the interaction between soluble and membrane-embedded subunits, supporting their combined isolation (Puts et al., 2010). Anyway, an examination of at least the hydrophilic stretches of membrane subunits and of the associated solvent-exposed subunits should be feasible (Fig. 15). Indeed, crosslinking was fruitfully applied to investigate the effect of serine/threonine/tyrosine (de)phosphorylation on the structure of chloroplast $\mathrm{F}_{1} \mathrm{~F}_{\mathrm{O}}$-ATP synthase (Schmidt et al., 2013). By successive dephosphorylations combined with crosslinking, the authors could accurately probe the changes in subunit interactions and distances resulting from (de)phosphorylation, providing strong evidence for their role in the structural integrity and catalytic action of ATP synthase.

\subsubsection{Protein-lipid interactions}

The lipid bilayer is vital for life. It not only separates the inside of the cell from the outside world, but it is also the site where respiratory processes are localised. It has become increasingly clear that close interaction between respiratory RMPs and lipid molecules is essential for the proper structuring and activity of these RMPs (Yeagle, 2013; Martfeld et al., 2015; Stangl and Schneider, 2015). Cardiolipin and other anionic lipids play a key role in this respect, both in mitochondria (Claypool, 2009; Hasan et al., 2011; Paradies et al., 2014) and in bacterial respiratory systems (Arias-Cartin et al., 2014). It has been known for a long time that the loss of cardiolipin results in the loss of activity of bacterial membrane-bound enzymes such as NDH, nitrate reductase NarGHI, formate dehydrogenase, succinate dehydrogenase, alpha-ketoglutarate dehydrogenase, quinol:cytochrome $c$ oxidoreductase (complex III) and cytochrome boз:ubiquinol oxidase (van Gestel et al., 2010; Arias-Cartin et al., 2012). 
Supplementation with cardiolipin restores these activities. Furthermore, tight, non-covalent, yet highly specific spatial associations between membrane-embedded subunits and cardiolipin are found in crystal structures of RMPs (Arias-Cartin et al., 2011, 2012; Planas-Iglesias et al., 2015).

Cardiolipin is not evenly distributed across the cell membrane, but it is dynamically accumulated at the poles and the division centre of rod-shaped bacterial cells (Oliver et al., 2014). Intriguingly, nitrate reductase is mainly found at the poles as well, consistent with the partnership between protein and lipid molecules (Alberge et al., 2015). Obviously, this uneven placement has its consequences for the spatial organisation of respiratory systems and concurrent bioenergetic processes (Alberge et al., 2015).

The immediate implication of the above considerations for structural proteomics on respiratory RMPs is that the field has to take protein-lipid interactions into account. That is to say, investigations should not only be restricted to peptides but should also include the identification and quantification of lipid molecules. This requires a close collaboration between proteomics and lipidomics, which means lipid analysis by mass spectrometry (Kliman et al., 2011). In the ideal case, methods should become available to precisely probe the interaction sides between protein and lipid. One such way is the ground-breaking methodology described in next section.

\subsubsection{Membrane protein complexes studied in thin air}

One of the most amazing and counterintuitive recent discoveries in proteomics of RMPs was that these proteins may not only be studied in their native lipid environment (or once purified, in artificial solvent systems), but also in gas phase and under vacuum (Laganowsky et al., 2013). Just like soluble proteins, an RMP present in appropriate micelles (Barrera et al., 2008; Calabrese et al., 2015) can get volatised and ionised, despite the low charge state associated 
with hydrophobicity. In this volatilised state, the native complex remains intact with all interactions between subunits, substrates, lipids and its other partners (Morgner et al., 2012; Hopper and Robinson, 2014; Zhou and Robinson, 2014; Chorev et al., 2015; Landreh and Robinson, 2015). And just like for soluble proteins, upon increase of ionisation energy all binding partners consecutively disintegrate into sub-complexes, individual subunits still carrying PTMs, lipid molecules and cleaved protein products. Subsequently, all of these components can be identified, quantified and sequenced (Fig. 14). By combined peptide and lipid analyses, the foundation has been paved for the in-depth elucidation of the RMP-lipid interplay (Barrera et al., 2013; Borysik et al., 2013; Hopper et al., 2013).

In addition to the rapid development of other top-down native MS techniques, another recently introduced powerful tool is ion-mobility MS (IM-MS) (Lanucara et al., 2014; Allison et al., 2015; May and McLean, 2015). In this method, ionised proteins are introduced into a cell filled with inert gas. Across this cell an electric field is maintained. Herein, proteins (and other molecules present in the sample) collide. The collision frequency depends on surface size: the larger the surface, the higher the number of collisions. Driven by the electric field, charged molecules exit the cell, somewhat resembling liquid capillary electrophoresis. This diffusion rate ("drift time”) is directly related to the size and charge of the molecule: molecules with the highest mass migrate slowest. The result is a series of elution profiles of the individual molecule species. After exit, molecules are analysed in the MS part of the instrument and quantified. When calibrated with proteins of known structures, collected data allows the calculation of the average collisional cross section of a protein (CCS in $\AA^{2}$ ) providing important 3D structural information. In addition, this data enable the calculation of dissociation constants of binding partners as well as the revelation of subtle conformational changes and ligand-binding-induced unfolding patterns (Rabuck et al., 2013; Stojko et al., 2015; Laganowsky et al., 2014; Niu and Ruotolo, 2015). Thus, by IM-MS information is 
gathered on the 3D structure and on the dynamics of a protein. A particular nice example of the opportunities offered by native MS and IM-MS in combination with lipidomics is the work by Zhou et al. (2011). These authors investigated the V-type ATPases of Thermus thermophilus and Enterococcus hirae by these methods, which enabled the establishment of the subunit stoichiometries and the identification of the lipids that were associated with the rotor ring. Additionally, from analyses of sub-complexes formed in solution and in the gas phase, the effect of nucleotide binding on both ATP hydrolysis and proton translocation was assessed. In the later context of RMP assembly and maturation (Section 5.2.1) the application of IM-MS in unravelling the conformational dynamics between the NarJ chaperone and the NarG catalytic subunit of nitrate reductase should be noted (Lorenzi et al., 2012).

\subsubsection{Putting structural information in a timed perspective}

The application of bottom-up and top-down MS approaches and their variants, especially when performed by well-designed combinations, may give information at all four structural levels of a protein complex to the extent that a 3D model of it can be proposed (Waltzhoeni et al., 2013; Boersema et al., 2015; Marcoux and Cianférani, 2015). The MS-based model will not be as refined as one deduced from X-ray crystallography or NMR. However, these three approaches can be complementary (see f.i. Nyon et al., 2015). If refractory to X-ray or NMR analyses, MS structural data of a protein complex can be modelled onto cryo electron microscopy (EM) maps, as has been done to determine the molecular architecture of the proteasome of Schizosaccharomyces pombe (Lasker et al., 2012). Unlike X-ray crystallography, NMR and EM, structural proteomics brings one more dimension into play: time. Cooperative movements and structural changes are revealed over a timescale from milliseconds to minutes (Zhou and Robinson, 2014). Moreover, proteomics offers two more benefits. Firstly, purification or BNE under mild conditions may detect more interaction 
partners like SCs, assembly factors, regulatory proteins and other compounds that direct enzyme activity (see Section 5). Secondly, the MS-supported approach is strong in identifying PTMs (see next, Section 4.2). The challenge is to apply these proteomics tools on bacterial respiratory systems of which many known ones remain to be to be investigated in more detail and even more new ones may be discovered in the near future. The above examples of the work on the $\mathrm{F}_{1} \mathrm{~F}_{\mathrm{O}}$ - and $\mathrm{V}$-type ATPes, and nitrate reductase indicate that such an approach could be feasible.

\subsection{Post-translational modifications}

Proteins are composed of a standard collection of twenty different amino acids, but currently more than 450 ways are known in which the primary structure of these amino acids are modified in biological systems. Modifications are made after translation to serve proper folding, assembly and function (Ryšlavá et al., 2013). Generally, a modification results from the action of dedicated enzymes and other dedicated enzymes may remove PTMs, in order to recover the original amino acid. Reversible PTM provides a means to change enzyme activity instantaneously. PTMs originate also chemically from exogenous or endogenous sources like reactive oxygen (ROS) and reactive nitrogen species (RNS) that destroy proteins, requiring the controlled action of repair or protein degradation systems. Next to this, PTM lies at the basis of signalling pathways by which an organism responds to changes in its environment. Living systems have evolved a plethora of relatively simple to extremely complex signalling pathways that may communicate with each other ("crosstalk") to enable the appropriate response of the whole cell to an environmental change. Crosstalk may also occur at the level of the enzyme itself. A substantial percentage of enzymes has more than one and even many sites for a same PTM or for different PTMs (Young et al., 2010; Soufi et al., 2012; Venne et 
al., 2014). By differential modification, enzyme activity can be fine-tuned in response to different stimuli.

The process of (reversible) protein modification has been known for a long time, especially in eukaryotes that have exploited its possibilities in every conceivable variant. The true magnitude of this PTM universe has been revealed by proteomics (Olsen and Mann, 2013). Currently, tens of thousands of PTMs have been identified and localised within protein sequences. Proteomics made it even so clear that prokaryotes utilise a same arsenal of PTMs as Eukarya to control their cell processes (Grangeasse et al., 2015).

On several occasions, we pointed out difficulties in analysing and identifying PTMs by (standard) proteomics, especially since identification of each type of PTM requires its own strategy (see for reviews on methodological aspects: Zhao and Jensen, 2009; Young et al., 2010; Kettenbach et al. 2011; Černý et al., 2013). Consequently, identification of PTMs could only be addressed within the field of proteomics after the development of methods that enabled (1) the routine enrichment of modified peptides from complex biological samples, (2) robust MS analysis, and (3) analyses on a high throughput basis. In the following, we will give a general overview of prokaryotic research on protein phosphorylation, acetylation, methylation, and redox-related PTMs and we will refer to recent reviews that will give entry to the extensive primary literature. Specific examples are related to the topic of this volume, the regulation of bacterial electron transfer chains and of mitochondrial OXPHOS complexes where the impact of the different types of PTM is better comprehended.

\subsubsection{Protein phosphorylation}

For a long time, it was believed that the role of phosphorylation in the regulation of bacterial cell processes was limited to the phosphorelay two-component systems, the phosphotransfer system in the uptake of sugars (Stülke and Hillen, 1998), serine phosphorylation of the 
catabolite control protein A (ссрA) (Fujita, 2008), and in the regulation of the isocitrate dehydrogenase by the bifunctional AceK kinase/ phosphatase controlling the entry into the glyoxylate shunt (Zheng and Jia, 2010; Zheng et al., 2012). However, the introduction and application of MS-based methods (Gerber et al., 2003; Villén and Gygi, 2008; Olsen and Maček, 2009; Maček et al., 2009; Wang et al., 2014) for the global identification of eubacterial and archaeal phosphoproteomes demonstrated that this view was too narrow (Maček and Mijakovic, 2011; Mijakovic and Maček, 2012).

Even though histidine and aspartate phosphorylation acting in the two-component regulatory and phosphotransfer systems remained undetected because of the labile nature of the phosphoryl bonds, proteomics showed extensive phosphorylation of serine (S), threonine (T) and tyrosine (Y) residues in all investigated prokaryotes (Table 4) < Table $4>$. This table shows that the extent of phosphorylation is highly variable among different species with respect to the number of proteins and the number of verified phosphorylation sites. Although microbial species most certainly differ from each other in the degree and ways protein phosphorylation is employed in the regulation of cellular processes, these differences are also due to methodological flaws. Initially, phosphoproteomes were analysed by 2DE with which protein phosphorylation was visualised by fluorescent labelling by Pro-Q Diamond (Agrawal and Thelen, 2005). Unfortunately, the original method was rather imprecise, both regarding visual detection and MS identification. (Please note the recent improvement by Wang et al., 2014). An up to ten-fold higher recovery of phosphorylated proteins and identified phosphopeptides (“events”) was achieved by the use of shotgun proteomics (Maček et al., 2007) (Table 4). Herein, phosphopeptides from tryptic lysates are subsequently separated by strong cation exchange chromatography (SCX), enriched by binding to $\mathrm{TiO}_{2}$ beads or by immobilised metal affinity chromatography (IMAC), and analysed by high-accuracy MS to identify phosphopeptides in the low-femtomole range (Maček and Mijakovic, 2011). 
Alternatively, phosphopeptides are enriched by immunoprecipitation by phosphoserine, phosphothreonine or phosphotyrosine antibodies (Černý et al., 2013). Despite continual methodological improvements, due to differences in sample (pre)preparation phosphopeptide recoveries vary substantially even for the same organism (see f.i. E. coli in Table 4), (Lin et al., 2015). While microbial species differ in the extent of protein phosphorylation, they also differ in phosphorylation targets and quite interesting differences may be seen in the ratios between serine:threonine:tyrosine phosphorylation (Table 4), likely reflecting species-related variations in regulatory strategies.

The phosphorylation degree of a protein is determined by the antagonistic activities between ATP-dependent protein kinases targeting S, T or Y residues and phosphatases. For serine and threonine phosphorylation, prokaryotes employ Hanks-type kinases as also known for eukaryotes (Maček and Mijakovic, 2011; Cousin et al., 2013). Tyrosine phosphokinases belong to the BY-kinase family and are unrelated to those of Eukarya (Grangeasse et al., 2007), even though their catalytic mechanisms are similar (Alber, 2009). Prokaryotic protein phosphatases are generally homologous to eukaryotic ones (Pereira et al. 2011). However, it is largely unknown how those kinases and phosphatases are activated. The elucidation of these regulatory mechanisms and the identification of protein substrates of the kinases and phosphatases are topics of extensive research (Maček and Mijakovic, 2011; Ravikumar et al., 2014; Mijakovic and Deutscher, 2015). One of the outcomes of these research efforts is that different phosphorylation systems are able of crosstalk (Jers et al., 2011; van Noort et al., 2012; Shi et al., 2014). No matter the complete underlying picture, phosphoproteome inventories made it fully clear that protein (de)phosphorylation directs the regulation of many key cellular processes, including central metabolism and respiration (for further details see references listed in Table 4). 
Indeed, bacterial respiratory complexes are phosphorylated. In E. coli, 4 phosphorylation sites are found on the alpha subunit of anaerobic respiratory fumarate reductase (FrdA), one each on the NuoB and NuoI subunits of NDH, one on the NarG catalytic subunit of nitrate reductase next to three on NarL, one on the cydA subunit of the high-oxygen affinity alternative terminal oxidase CydAB (cytochrome $d$ :ubiquinol oxidase, cytochrome bd-I oxidase; Borisov et al., 2011), and seven on the ATP synthase (AtpA, 1; AtpD, 3; AtpF, 3) (Maček et al., 2008). These same respiratory complexes are also targeted in B. subtilis (NDH, 1 site; NarG, 1 site; CydAB, 1 site; ATP synthase, 4 sites, including 2 sites each on AtpA and AtpB). Also respiratory complexes of Klebsiella pneunomiae undergo phosphorylation, albeit in different fashions. Here, one site is seen on FrdA, two sites on the NuoI subunit of NDH, 4 sites on AtpB, 4 sites on CydA and no less than 10 sites on CydC, the putative subunit III of the CydAB complex. The structural and functional significance of these bacterial phosphorylations remains to be established, but it is interesting to note that, except for ATP synthase, these events address RMPs that operate under anaerobic or microaerophilic conditions. In mitochondria, protein phosphorylation is known to be of fundamental importance in the regulation of the activities, assembly and maintenance of the structural integrity of the OXPHOS complexes (Pagliarini and Dixon, 2006; Reinders et al., 2007; Kane and Van Eyk, 2009; Reiland et al., 2009; Valsecchi et al., 2013). Notably, multiple phosphorylations have been revealed and partially identified with regard to their roles in the complexes I (NDH), IV (cytochrome $c$ oxidase) and V (ATP synthase). Phosphorylation of complex I concerns the nuclear-encoded accessory subunits NDUFA7, NDUFA10, NDUFC2, NDUFS4, ESSS, and GRIM-19, which have been implemented with the assembly of the 45-subunit complex (Palmisano et al., 2007; De Rasmo et al., 2010; Vartak et al., 2014). Cytochrome $c$ oxidase is phosphorylated at no less than 18 positions facing both the matrix and intermembrane space of the mitochondrion (Helling et al., 2012a, 
b). At least a number of these reversible phosphorylations seem to deal with "allosteric ATP inhibition" involved in the control of an appropriate pmf and in preventing the formation of superoxide radicals (Helling et al., 2008, 2012a,b; Hüttemann et al., 2012). The ATP synthesis machinery itself is phosphoryl-targeted at several subunits, enabling the regulation of its activity (Kane and Van Eyk, 2009; Kane et al., 2010). In the aforementioned paper by (Schmidt et al., 2013), it was shown that the phosphorylation degree had a direct effect on subunit interactions that might play a role in complex assembly and/or enzyme activity. Phosphorylation of the mitochondrial OXPHOS complexes is mediated by protein kinases. At least some of the kinases are allosterically regulated by cyclic-AMP, which involves, among other ones, the action of a novel, mitochondrial-specific soluble adenylate cyclase (Valsecchi et al., 2013, 2014; De Rasmo et al., 2015; Nowak and Bakajsova, 2015). As mentioned, the role of (reversible) phosphorylation of bacterial respiratory complexes is not understood, no more than possible signalling pathways either.

\subsubsection{Protein acetylation}

Proteomics established that besides protein phosphorylation, protein acetylation is fundamental in the regulation of central cellular processes, also in prokaryotes (see for reviews: Soppa, 2010; Kim and Yang, 2010; Jones and O'Connor, 2011; Bernal et al., 2014; Hentchel and Escalante-Semerena, 2015). In order to make a global inventory of acetylation sites and -events (the "acetylome”) shotgun proteomics is currently supported by the selective immunoprecipitation of acetylated peptides by specific antibodies (Guan et al., 2010; Zhao et al., 2010; Hentchel and Escalante-Semerena, 2015). Using this methodology, the acetylomes of a number of bacterial species have been determined (Table 5) < Table 5>. These acetylomes comprised between 60 to over 600 different proteins in species-dependent ways, and these numbers may very well be underestimates. For example, a recent study on the 
acetylome of B. subtilis (Kosono et al., 2015) reported a 3.5-fold higher coverage than found earlier (Kim et al., 2013). Nevertheless, the width and scope of protein acetylation makes the phenomenon of at least equal importance as phosphorylation. Unlike Eukarya, irreversible Nterminal acetylation is relatively rare in Bacteria (Hentchel and Escalante-Semerena, 2015). Just as it is the case for eukaryotes (Zhao et al., 2010; Guan and Xiong, 2011; Kim and Yang, 2011; Shi and Tu, 2015; Tripodi et al., 2015), acetylation addresses transcription, translation, protein folding, amino acid- and nucleotide biosynthesis, and metabolism in prokaryotes (Jones and O'Connor, 2011; Bernal et al., 2014; Hentchel and Escalante-Semerena, 2015). Protein acetylation even appears to be the crucial mechanism to control the central carbon metabolism and the TCA cycle at the posttranslational level (Wang et al., 2010). In agreement herewith, fructose-1,6-bisphosphate aldolase in E. coli (Zhang et al., 2013) has no less than 11 acetylation sites, besides 6 succinylation sites, which represents another recently discovered PTM (Weinert et al., 2013; Kosono at al., 2015). E. coli glyceraldehyde-3-phosphate dehydrogenase harbours even 14 acetylation sites, next to 9 succinylation sites, and in isocitrate lyase, which lacks any of the latter, 11 sites are subjected to acetylation. In contrast, isocitrate dehydrogenase of this organism, the model enzyme of bacterial protein phosphorylation, is acetylated on 5 positions, whereas phosphorylation occurs at 4 positions. In fact, a large proportion of bacterial enzymes has both phosphorylation and acetylation sites. The availability of PTMs in different modes and numbers provides the opportunity of crosstalking (Soufi et al., 2012; van Noort et al., 2012)). It remains to be experimentally investigated how and to what extent this occurs.

Protein acetylation usually concerns the $\varepsilon$ amino group of lysine to which acetylCoenzyme A is the acetyl donor. The reaction is catalysed by lysine acetyltransferases and in bacterial genomes three different classes of this enzyme are found (Jones and O'Connor, 2011; Bernal et al., 2014; Hentchel and Escalante-Semerena, 2015). It should be noted that 
lysine acetylation is also performed non-enzymatically by acetyl phosphate, but its physiological relevance is not fully understood (Kuhn et al., 2014; Wagner and Hirschey, 2014). Removal of the acetyl group is achieved by lysine deacetylases that are represented by four different classes of enzymes. By the presence of lysine acetyltransferases and deacetylases, the process is reversible and is prone to regulation. Bacterial genomes usually harbour a number of candidates of either enzyme (Hentchel and Escalante-Semerena, 2015). Yet, except for some cases (Xu et al., 2011; Nambi et al., 2013; reviewed in Hentchel and Escalante-Semerena, 2015), it is again far from clear which acetyltransferase or -deacetylase targets which set of substrates.

Concerning respiratory complexes, in E. coli NDH is acetylated at the NuoF (1 site) and NuoE (2 sites) subunits, at terminal oxidase CydAB on one site each of both subunits, at the AtpA (2 sites) and AtpB (3 sites) subunits of ATP synthase, at one site of nitrate reductase NarG, and at four different positions of FrdA (Zhang et al., 2013). One may note that the very same target proteins were found for phosphorylation (see Section 4.2.1), which again might suggest regulatory cross-talking. Once more, the structural, regulatory or functional implications of those acetylations are elusive. For the sake of comparison, more than one-third of all mitochondrial proteins are acetylated, most of these being involved in some aspect of energy metabolism (Anderson and Hirschey, 2012; Papanicolaou et al., 2014). Regarding mitochondrial complex I, thirteen of its subunits, all being nuclear-encoded, are acetylated at their N-terminal position (Carroll et al., 2005). ATP synthase is acetylated at evolutionary conserved positions on the $\mathrm{F}_{1}$ alpha, beta and gamma subunits as well as on the oligomycin sensitivity-conferring protein (OSCP) (Vassilopoulos et al., 2014). Deacetylation is catalysed by the $\mathrm{NAD}^{+}$-dependent SIRT3 sirtuin. In cells lacking functional sirtuin, ATP synthase remains acetylated and is impaired in its activity (Wu et al., 2013; Vassilopoulos et al., 2014). The role of SIRT3 is not restricted to ATP synthase. If absent or inactive, many mitochondrial 
proteins become hyperacetylated. Herewith, SIRT3 might turn out to be a key modulator of energy homeostasis (Anderson and Hirschey, 2012; Osborne et al., 2014). Sirtuins are also found in genomes of prokaryotes (Hentchel and Escalante-Semerena, 2015), suggesting a universal function of these proteins in PTM by reversible acetylation (Choudhary et al., 2014). In mycobacteria, a homolog already has also been identified as a regulator of a variety of enzymes (Xu et al., 2011; Gu et al., 2015). It is unknown whether sirtuins, or other lysine acetylases and deacetylases, act in the regulation of bacterial respiratory enzyme complexes.

\subsubsection{Protein methylation}

Besides acetylation, lysine can be post-translationally modified by mono-, di- and trimethylation. The other amino acids that can be added methyl groups are arginine, glutamine, aspartate and histidine. Two broad classes of enzymes are able to methylate amino acids and both use S-adenosylmethionine as a methyl donor (Schubert et al., 2003). The first class (formally Class V methyltransferases) is characterised by a so-called SET domain and these enzymes predominantly methylate histones in eukaryotes. Members of the second class (formally Class I methyltransferases), the seven $\beta$-strand methyltransferases, methylate DNA, RNA and the aforementioned amino acids. Lysine methylation is reversible, but bacterial demethylases are less well defined (Lanouette et al., 2014). Protein methylation plays a diverse role in biosynthesis, signal transduction, protein repair, chromatin regulation and gene silencing (Schubert et al., 2003; Lanouette et al., 2014), even though the chemistry of a group is only little effected by methylation. However, the presence of a methyl group may protect a protein from being modified by another PTM, like ubiquitination in eukaryotes (Pang et al., 2010). Next, lysine methylation creates a binding surface for the recruitment of other proteins or peptides. Amino acid methylation can also have a structural role in a protein itself. In methylcoenzyme $\mathrm{M}$ reductase (MCR) from methanogens, for example, histidine, arginine, 
glutamine and cysteine moieties close to the factor $\mathrm{F}_{430}$ catalytic centre in the alpha subunit are all methylated (Ermler et al., 1997). However, 2DE analysis (Fig. 9B) shows that the alpha subunit both in type I and type II MCR occurs in different proteoforms, possibly representing variations in these methylations.

While protein methylation addresses many cellular functions, application of proteomics to explore these functions on a genome-wide basis has lagged behind (Lanouette et al., 2014). There are several reasons for this delay. Firstly, it took a while before antibodies were found to enrich low-abundant methylated peptides specifically and with high affinity (Moore et al., 2013; Carlsson et al., 2014). Secondly, methylated peptides separate poorly on SCX-LC columns. Thirdly, the peptides are highly charged under ESI, which limits the number of sequence-informative products produced by CID, and loss of the labile methylation moieties during CID precludes effective fragmentation of the peptide backbone (Wang et al., 2009; Snijders et al., 2010). The latter problem has been solved by high-resolution ETD and $\mathrm{MS}^{3}$ methods (Wang et al., 2009; Snijders et al., 2010). By the application of one of these techniques, the identification of mono-, di- or trimethylated amino acids in purified or highly enriched protein preparations that harbour those species structurally shouldn't be a problem, considering mass shifts of 14, 28 and 42 Da. (Note, however, the very small mass difference between trimethyllysine, $42.05 \mathrm{Da}$, and acetyllysine, $42.01 \mathrm{Da}$ ). We will illustrate these applications in view of mitochondrial OXPHOS complexes.

The analysis of whole complex I revealed a complex PTM pattern of subunit B12 that forms part of the membrane arm (Carroll et al., 2005). Its $\mathrm{N}$-terminus is a mixture of acetylated and free amino acids, whereas one, two or three methyl moieties are attached to histidine residues 4, 6 and 8 in various combinations. However interesting, the physiological significance of this complexity is not understood. Next, arginine 82 of the $42-\mathrm{kDA}$ subunit (NDUFS2) is symmetrically dimethylated on the $\omega-\mathrm{N}^{\mathrm{G}}$ and $\omega-\mathrm{N}^{\mathrm{G}^{\prime}}$ nitrogen atoms of the 
guanidine group (Carrol et al., 2013). Arginine 82 is localised near the quinone-binding site and its position is conserved in yeast and in Paracoccus denitrificans, suggesting that this PTM is functionally important. Dimethylation is catalysed by the assembly factor NDUAF7 (Rhein et al., 2013) and this methylation step occurs early in complex I assembly. Another interesting example is ATP synthase. One of its key elements is the rotor ring composed of 75 amino acid c-subunits, each crossing the membrane twice. TMHs are linked by a short loop that contains a lysine (K43). In metazoans, this lysine is trimethylated, which might provide a site for cardiolipin binding (Walpole et al., 2015). Importantly, in metazoans the rotor ring is comprised of eight c subunits. In unicellular Eukarya and prokaryotes, the lysine is either not methylated or substituted for another amino acid. The rotor ring of these organisms harbours 9-15 c subunits. This small difference in lysine substitution has very important bio-energetic consequences for the $\mathrm{H}^{+}$translocation/ ATP synthesis ratio: each c subunit takes one proton across the membrane per rotor rotation at which 3 molecules of ATP are synthetized. Hence, the more c subunits are present in the rotor ring, the less ATP is made per rotation. A direct implication for the latter is that ATP is still feasible at lower pmf.

\subsubsection{Oxidative stress-related post-translational modifications}

In their natural environment or during infections, bacteria are exposed to what is collectively called oxidative stress. This stress is caused by reactive oxygen (ROS), nitrogen (RNS), chlorine (RCS) or electrophilic (RES) species (Antelmann and Helmann, 2011; Loi et al., 2015). Hosts use these species to bombard and kill their infectious invaders. However, oxidative stress is also endogenous during of respiration. ROS include superoxide $\left(\mathrm{O}_{2}^{-}\right)$, hydrogen peroxide $\left(\mathrm{H}_{2} \mathrm{O}_{2}\right)$ and the most detrimental of all, the hydroxyl radical $(\mathrm{OH} \cdot)$ (Bleier et al., 2015; Imlay, 2015). These species are formed by successive one-electron reductions of dioxygen, which are the result of side reactions of respiratory enzymes or are catalysed by 
enzymes that are intended to remove ROS, such as superoxide dismutase, catalase, cytochrome $c$ peroxidase and NADPH-dependent alkyl hydroperoxide reductase (Fig. 16A). In respiratory enzymes, reactions of oxygen with iron-sulphur clusters, (semi)reduced flavins or quinones are a major source of superoxide and $\mathrm{H}_{2} \mathrm{O}_{2}$. Hydroxyl radicals are nonenzymatically produced by the reaction of $\mathrm{H}_{2} \mathrm{O}_{2}$ with $\mathrm{Fe}^{2+}$, better known as the Fenton reaction (Imlay, 2015). The major RNS are nitric oxide (NO) and peroxynitrite, which is formed by the reaction between NO and oxygen. RNS are generated endogenously during anaerobic nitrate respiration or they are actively produced as an antimicrobial agent by activated neutrophils. These neutrophils are also producers of the predominant RCS, hypochloric acid ( $\mathrm{HOCl}$, bleach), which is a strong oxidant $\left(\mathrm{E}_{0}{ }^{\prime}=1.28 \mathrm{~V}\right.$ ) (Hillion and Antelmann, 2015; Loi et al., 2015). RES are comprised of broad collection of compounds, like quinones, methylglyoxal and diamide, that react with suitable nucleophiles, most notably with the thiol (SH) group of cysteine, via (irreversible) thiol-S-alkylation chemistry.

Reactive oxygen and other species have a detrimental effect on DNA, lipids and proteins. The compounds cause lesions in proteins and the destruction of mononuclear iron centres and iron-sulphur clusters involved in catalysis or electron transfer (Imlay, 2015). Cysteine is particularly sensitive towards these species. Upon reaction with ROS, its thiol group is first oxidised to the unstable cysteine sulphenic acid (R-SOH), which reacts with other thiols to form either intra- and intermolecular protein disulphides (RS-SR') or heterodisulphides with low-molecular-weight (LMW) thiols in the cell (Antelmann and Helmann, 2011; Hillion and Antelmann, 2015; Loi et al., 2015)(Fig. 16B). Disulphide formation is reversible and this reversibility is the basis of protein repair. Cysteine sulphenic acid readily oxidises to sulphinic $\left(\mathrm{RSO}_{2}{ }^{-}\right)$and sulphonic $\left(\mathrm{RSO}_{3}{ }^{-}\right)$acid and these oxidations are irreversible. Also NO and $\mathrm{HOCl}$ rapidly react with cysteine, causing cysteine $S$-nitrosation (RS-NO') and cysteine sulphenylchloride (RS-Cl) formation, respectively. The latter 
compounds are also unstable and reaction with other thiols forms disulphides. In the absence of these thiols, nitrosated cysteines and cysteine sulphenylchlorides are further irreversibly oxidised to sulphinic and sulphonic acid.

Microorganisms have developed ingenious systems to cope with oxidative stress and they do this by a diversity of regulatory systems that have been appreciated only recently (see for reviews: Antelmann and Helmann, 2011; Fu et al., 2015; Hillion and Antelmann, 2015; Loi et al., 2015). Best known are the OxyR and SoxR systems of E. coli, which protect the organism from peroxide and superoxide stress, respectively (Chiang and Schellhorn, 2015; Imlay, 2015). The common property of all those systems is the presence of transcriptional regulators that both sense specific stressing agents at very low concentrations, and in response activate enzyme machineries that clear those agents and repair the damage. In order to sense, the regulators exploit the same chemistry just described. Reaction with a stressing compound elicits a conformational change of the regulator, which is translated in protein expression. For instance, after reaction with hydrogen peroxide or with NO two specific cysteines in OxyR make a disulphide bond, causing the concomitant structural change of the protein (Jo et al., 2015). In SorR, a $\mathrm{Fe}_{2} \mathrm{~S}_{2}$ cluster is oxidised upon reaction with superoxide, leading to dimerization and activation of this regulator (Kobayashi et al., 2014). Key components in the protection against oxidative stressors are LMW thiol compounds, familiarly termed redox buffers. The roles of these thiols are dual. Firstly, by chemical reaction with a stressing agent, this agent is eliminated at which the thiol is oxidised to its homodisulphide. After reduction catalysed by a (NADPH-dependent) disulphide reductase, the thiol is regenerated for a next reaction. Secondly, thiols react with cysteine sulphenic acids in oxidatively damaged proteins (Fig. 16B) resulting in the formation of cysteine heterodisulphides (“protein-S-thiolation”), but protecting these cysteines from further oxidation. Enzymatic reduction of the disulphide bond recovers the original cysteine(s) in the protein. Bacteria harbour a number of these 
thiols, the simplest one being cysteine itself (Fahey, 2013). However, the disadvantage of cysteine is that it undergoes a heavy-metal-catalysed auto-oxidation at considerable rate and at which ROS are formed. Such auto-oxidation of the tripeptide glutathione (GSH, $\gamma$-Lglutamyl-L-cysteinylglycine) is two orders of magnitude slower and GSH is used by Gramnegative bacteria and in eukaryotes as a redox buffer. Firmicutes lack GSH, but have bacillithiol (BSH, L-cysteinyl-D-glucosamine-malate) instead, whereas Actinobacteria employ mycothiol (MSH, N-acetyl-L-cysteinyl-D-glucosamine-inositol). It cannot be ruled out completely that also coenzyme A serves this purpose to an extent. Thus, oxidative damage of proteins is observed as protein-S-thiolation by GSH, BSH, MSH or just cysteine, and this thiolation supports for the global mapping of oxidative stress. For this mapping proteomics comes into play.

Several methods have been developed to make a whole-cell analysis of proteins that underwent PTMs due to oxidative stress. These methods follow the 2DE and shotgun methodologies described before (see Sections 3.1-3.3) with specific modifications (reviewed in Leichert and Jakob, 2006; Lindahl et al, 2012; Černý et al., 2013; Loi et al., 2015). Briefly, a protein extract of ROS-exposed cells is reduced by an appropriate reagent (f.i. ascorbate for nitrosation, arsenite for sulphenylation, tris(carboxyethyl)phosphine for general disulphides, specific enzymes to reduce RS-SGH, RS-BSH RS-MSH disulphides). Cysteine moieties in proteins formed after reduction are fluorescently labelled and the enzyme mixture is separated by 2DE for further MS analyses (Leichert and Jakob, 2004). By using two different fluorescent dyes, DIGE immediately reveals the (quantitative) difference in protein patterns between controls and ROS-exposed cells. Alternatively, reduced extracts are labelled with thiol-reactive biotin and biotinylated proteins are visualised by anti-biotin immunoblotting. Biotin labelling provides also the means for selective enrichment by streptavidin affinity chromatography. Proteolytic cleavage before or after enrichment enables bottom-up shotgun 
proteomics of the protein preparations; furthermore, there are also methods to differentially quantify PTMs (Lindemann and Leichert, 2012). These procedures allow the identification of modified proteins, but the information regarding the nature of protein-S-thiolation is obviously lost. Consequently, shotgun analyses have to be run in parallel on preparations that have not been biotinylated, hoping the find candidate proteins with molecular masses that account for a substation by a LMW thiol.

The above proteomics methods have been applied to uncover the global thiol-based redox regulation in a number of bacterial species (see f.i. Hochgräfe et al., 2007; Pohl et al., 2011; Schroeter el al,. 2011; Chi et al., 2011, 2013, 2014; Lindemann et al., 2013; Kim et al., 2013). Recently, Ansong et al. (2013) used a top-down approach to map a S-thiolation switch on whole proteins from Salmonella typhimurium in response to infection-like conditions. Protein preparations obtained from cells grown under different conditions were separated on a long $(80 \mathrm{~cm})$ nanocapillary column and an extended (250-min) gradient to prevent undersampling for subsequent Orbitrap MS. By this method, 1665 S-thiolation sites on 563 unique proteins were detected. Intriguingly, the S-thiolation mode switched between Scysteinylation and S-glutathionylation depending on the growth condition. Also respiratory enzymes were targeted by $S$-thiolation and these comprised the CydAB terminal oxidase (one site each on CydA and $\mathrm{CydB}$ ), three sites on the DmsB subunit of dimethylsulphoxide oxidoreductase and no less the eight positions on the cyt $b_{562}$ subunit of quinone:cytochrome $c$ oxidoreductase (complex III). It may be worth mentioning that in C. glutamicum CydB expression in stands under control of the OxyR system (Teramoto et al., 2013). ATP synthase was particularly sensitive towards PTM resulting from oxidative stress, being modified at AtpA (6 sites), AtpC (4), AtpD (7), AtpG (3), AtpF (1) and AtpH (1).

In mitochondria, $0.2-2 \%$ of all oxygen respired ends up in ROS as the result of the activities of complexes I, II and III. Therefore, it will be no surprise that many enzymes in this 
cell organelle are targeted by ROS and that elaborate regulatory systems are available to protect and repair proteins in response to oxidative stress (see for a review: Dröse et al., 2014). However, proteomics revealed that protection could proceed in quite unexpected manners. Several of subunits of complex I are targets of oxidative thiol-modification with one being of special interest. In vitro and in vivo, vertebrate complex I occurs as two conformers, to so-called active (A) and deactive (D) state (Brandt, 2011). The A-state is catalytically competent and the D-state is a dormant one, which is taken when the complex is devoid of its substrates (NADH, ubiquinone). The presence of these substrates induces the transition of the

D- into the A-state: So, A/D transition is reversible (Babot et al., 2014). This transition is also known for complex I of unicellular Eukarya, but this has not been established for prokaryotic NDHs thus far. Only in the D-state, a specific cysteine is accessible for artificial modification by N-ethylmaleimide or iodoacetamide, or physiologically by nitrosation by NO (Galkin et al., 2008). This particular cysteine (Cys-39) is localized in the ND3 subunit, close to the quinone binding site. If Cys-39 is S-nitrosated, complex I is kept in the D-state and is consequently unable of turnover, preventing the formation of excess ROS. By the removal of NO (or reduction of an S-glutathionylated form) catalytic activity is restored eventually (Prime et al., 2009; Chouchani et al., 2013); nevertheless, it is still elusive how this is done.

\section{ASSEMBLAGES OF RESPIRATORY PROTEIN COMPLEXES}

\subsection{Supercomplexes}

\subsubsection{Mitochondrial supercomplexes}

The introduction of BNE into the research on respiratory systems by Schägger and colleagues (1988, 1991, 2000, 2002) changed our thinking on the way these systems are organized. Until these publications, the accepted idea was that respiratory protein complexes moved laterally and independently from each other along the membranes ("liquid state model”), a view that is 
still common in many textbooks (Fig. 17A). However, BNE analyses revealed that mitochondrial OXPHOS complexes may aggregate as large functional "supercomplexes" (SC), as already had been suggested earlier (“solid state model”) (Schägger, 2002). Initially, the SC concept was met with scepticism. It was argued that aggregation was the result of experimental artefacts that arise from membrane resolution and subsequent BNE of respiratory complexes. This objection is valid and should always be kept in mind when performing such experiments. Nevertheless, original results could be repeated by many labs and by application of BNE and CNE as outlined in Section 3.2.2, and SCs have since been detected in mitochondria of animals, plants (Eubel et al., 2004; Krause et al., 2004; Bultema et al., 2009; Klodmann et al., 2011), yeasts (Lemaire and Dujardin, 2008; Helbig et al., 2009; 2009; Nübel et al., 2009; Matus-Ortega et al., 2015), as well as in respiratory systems of prokaryotes (see the following section). Furthermore, SC formation is also observed for photosystems (Watanabe et al., 2011, 2014; Cartron et al., 2014; Chang et al., 2015). Importantly, ideas regarding their organization based on proteomics could be confirmed independently by high-resolution electron microscopic studies (Schäfer et al., 2007; Vonck and Schäfer, 2009; Dudkina et al., 2010; Chaban et al., 2014). By now, there is little doubt that SCs play somehow a critical role in respiratory processes (Wittig et al., 2006b; Shoubridge, 2012; Vartak et al., 2013; Acín-Pérez and Enriquez., 2014).

In mitochondria, complexes I, III and IV form SCs in different combinations (Fig. 17B). Complex II (SDH) and complex V (ATP synthase) do not take part these SCs. In fact, ATP synthase molecules assemble as SCs by themselves (Schäfer et al., 2007; Vonck and Schäfer, 2009; Wittig and Schägger, 2009b; Dudkina et al., 2010; Chaban et al., 2014). The basic unit is the ATP synthase dimer (Wittig et al., 2008; Wittig and Schägger, 2009b) at which the monomeric forms are arranged such that they make angles between 30 and 145 degrees with respect to each other (Chaban et al., 2014) (Fig. 17C). Angles vary between 
different organisms and they may change when the protein is resolved from the mitochondrial membrane. In yeast, dimerization is controlled by the phosphorylation state of the ATP synthase (Bornhövd et al., 2006; Reinders et al., 2007; Kane et al., 2010). In turn, ATP synthase dimers are arranged in parallel to make long strings that sit on top of the cristae and bend the membrane, thus shaping this inner membrane system (Dudkina et al., 2005b; Davies et al., 2012). This parallel arrangement is facilitated by dimer-specific, non-catalytic subunits e and g (Bornhövd et al., 2006; Saddar et al., 2008; Wittig and Schägger, 2009b). The planar parts of the cristae provide space for the respiratory complexes (Cogliati et al., 2013). BNE and CNE analyses of mitochondria of representatives of all Eukarya conclusively showed that these complexes formed SCs composed of individual complexes in more or less fixed ratios, viz. I+III ${ }_{2}, \mathrm{III}_{2}+\mathrm{IV}_{1-2}, \mathrm{I}+\mathrm{III}_{2}+\mathrm{IV}_{1-4}$ (Fig. 17B) (Chaban et al., 2014). The different SCs have been purified in their native states and they all showed the expected activities (Acín-Pérez et al., 2008). SC $\mathrm{I}+\mathrm{III}_{2}$, for instance, is capable of cytochrome $c$ reduction by NADH, and $\mathrm{I}+\mathrm{III}_{2}+\mathrm{IV}_{1-4}$ ("respirasome") catalyses the NADH-dependent reduction of oxygen. The SC $\mathrm{I}+\mathrm{III}_{2}+\mathrm{IV}_{1-4}$ seems to be the building unit of long strings or patched strings that stretch and spread along the cristae (Wittig and Schägger, 2009b; Dudkina et al., 2010; Muster et al., 2010). The resulting straightforward questions are: (1) what is the function of the SCs and (2) how are these SCs assembled, assuming that assembly is a directed process.

Based on ample experimental evidence, a number of functions have been proposed for SCs, but none of them provide a definite answer as yet. By the tight control of electron carrier interchange (viz. ubiquinone and cyt $c$ ) in between the individual complexes, formation of ROS would be minimized (Rosca et al., 2008; Gómez et al., 2009; Maranzana et al., 2013). The close association between the individual complexes would decrease the distances that electron carriers have to travel, thereby increasing the electron flux ("substrate tunnelling”) (Vartak et al., 2013; Chaban et al., 2014; Acín-Pérez and Enriquez., 2014). Indeed, this 
electron flux is governed by SC assembly (Lapuente-Brun et al., 2013). Moreover, structural models made on basis of electron microscopy and known crystal structures indicate a short distance between the cytochrome $c$ binding sites of complex III and IV (Schäfer et al., 2007), and a SC composed of the latter two was shown to contain a stoichiometric amount of 2 cytochrome $c$ molecules (Moreno-Beltrán et al., 2015). However, such a short distance is less apparent for the quinone-binding sites of complex I and III (Dudkina et al., 2005a), and quinone was shown to move freely through the mitochondrial inner membrane during respiration (Lenaz and Genova., 2009; Trouillard et al., 2011; Genova and Lenaz, 2013, 2014; Blaza et al., 2014). Another possibility is that individual complexes cooperatively assist and stabilize each other's assemblies during SC formation and maintenance (Stroh et al., 2003; Marquez et al., 2007; Saddar et al., 2008; Duarte and Videira, 2009; Calvaruso et al., 2012; Habersetzer et al., 2013; Cui et al., 2014; Chojnacka et al., 2015; see however: Maas et al., 2009). Indeed, deletion or mutation of factors known to be involved in the assembly of one complex affected assembly of other complexes as well and of SCs as a whole. Presently, three factors are known to assist in SC formation as such, stomatin-like protein 2 (Mitsopoulos et al., 2015), metalloprotease OPA1 (Bohovych et al., 2015) and dynamin-related protein OMA1 (Cogliati et al., 2013) at which genetic deletion of the latter results in the complete disorganization of the cristae (Frezza et al., 2006). Besides these proteinaceous factors, one more component is essentially for at least the structural integrity of mitochondrial SCs, cardiolipin (Zhang et al., 2002, 2005; Wenz et al., 2009; Bazán et al., 2013; Mileykovskaya et al., 2014; Desmurs et al., 2015).

Thus, it is obvious that the function of mitochondrial SCs is not fully comprehended and that assemblage is a highly complicated and even so poorly understood interplay of a multitude of assembly factors. In addition, SCs are found as different aggregates (Fig. 17B) and their composition may change depending on the growth conditions applied (Schägger and 
Pfeiffer, 2000; Ramírez-Aguilar et al., 2011). Perhaps, transitions in between the various SCs are dynamic, as proposed in the plasticity model by Acín-Pérez and Enriquez. (2014), providing an additional level of control of respiratory processes and at which control could even place at a very local scale.

\subsubsection{Bacterial supercomplexes}

BNE/ CNE and other biochemical studies established that SC formation is also common to prokaryotic ETCs (Magalon et al., 2012; Melo and Teixeira, 2015), where the composition of the SCs reflects the respiratory status. In Paracoccus denitrificans, the presumed progenitor of mitochondria, the main SC is comprised of NDH (complex I), the $b c_{1}$ complex (complex III) and the cytochrome $a a_{3}$ oxidase (complex IV), just like the respirasomes in mitochondria (Stroh et al., 2004). The thermophilic bacterium Aquifex aeolicus that is able of aerobic growth with $\mathrm{H}_{2} \mathrm{~S}$ as the electron donor, harbours a SC composed of one or two sulfide:quinone oxidoreductases, one dimeric $b c_{1}$ complex, and one $b a_{3}$-type terminal oxidase, next to traces of quinones and monoheme cytochrome $c_{555}$ (Prunetti et al., 2010). As-isolated this SC is able indeed to couple $\mathrm{H}_{2} \mathrm{~S}$ oxidation with oxygen reduction. Similarly, a supermolecular structure isolated from the acidiphilic bacterium Acidithiobacillus ferroxidans that spans two membranes and that is constituted of iron-oxidising proteins and cytochrome $c$ oxidase $a a_{3}$, catalyses $\mathrm{Fe}^{2+}$ oxidation with oxygen reduction when supplemented with the physiological electron carrier cytochrome $c_{2}$ (Castelle et al., 2008). By streptag-affinity chromatography a SC has been purified from aerobic Gram-positive C. glutamicum, which is assembled from a $b c_{1}$ complex, a diheme cytochrome $c_{1}$ electron carrier and cytochrome $c a a_{3}$ oxidase, containing a hitherto elusive fourth subunit (Niebisch and Bott, 2003). The complex had quinol oxidase activity as expected. A similar SC has been obtained from B. subtilis (Sousa et al., 2013b). Here, the bc:aa 3 complex was observed upon BNE in different ratios, 
$(b c)_{4}:\left(c a a_{3}\right)_{2},(b c)_{2}:\left(c a a_{3}\right)_{4}$, and 2[(bc) $\left.4:\left(c a a_{3}\right)_{2}\right]$, indicative of string formation. BNE allowed the detection of one more interesting SC, a succinate:quinone oxidoreductase:nitrate reductase. One may note, that SDH (complex II) never formed part of mitochondrial SCs. The presence of the succinate:nitrate oxidoreductase and $b c: a a_{3}$ SCs in $B$. subtilis was substantiated in another study (García Montes de Oca et al., 2012), where it was shown that also membrane-bound cytochrome $c_{550}$ was a component and the electron carrier of the $b c: a a_{3}$ SC. Intriguingly, this SC was associated with ATP synthase monomers. Next, a stable hybrid bc:aa3-type SC has been artificially assembled from two different Mycobacterium species (Kim et al., 2015). The presence of $b c: a a_{3}$ SCs in Gram-positive bacteria is highly reminiscent to the complex III:complex IV SCs found in mitochondria (Fig. 17B). Some Gram-negative bacteria, like E.coli, lack complex III, but these organisms have their share in SCs as well, supporting the various respiratory pathways that enable these organisms to grow under varied environmental conditions (Sousa et al., 2011, 2012, 2013a,b). By BNE and CNE combined with kinetic data of wild type and mutant strains and transcription analyses, these authors identified a number of SCs, including a trimer of SDH, a direct association between the two types of NDH found in E. coli, the canonical multisubunit NDH-1 and more simple flavin NDH-2, and SCs composed of the aerobically stable formate dehydrogenase (fdo) together with two oxidases that $E$. coli has the disposal of cytochrome boз quinol:oxygen reductase and cytochrome $b d$ quinol:oxygen reductase (CydAB).

Like in mitochondrial research, BNE and CNE supported by MS provide a strong method to analyse prokaryotic SCs. Accessory subunits and low-molecular weight electron carriers are preserved in RMPs by mild detergent-treatments of the membrane. They had gone unnoticed in classic biochemical purifications, as exemplified above. The oxygen-stable, membrane-bound [NiFe]-hydrogenase (MBH) from the 'Knallgas' bacterium Ralstonia eutropha examplifies such loss very well (Frielingsdorf et al., 2011). The enzyme has been 
well-characterised, but the as-isolated protein always lacked one candidate subunit found in the genome, which would bind MBH to the membrane and shuttle electrons into the ETS, viz. a heme $b$ subunit. Preparations only contained this heme $b$ subunit after mild extraction with digitonin and, unexpectedly, $\mathrm{MBH}$ appeared to be a homotrimeric SC. The lipids phosphatidylethanolamine and phosphatidylglycerol were indispensable to keep interaction between the soluble hydrogenase module and the membrane-bound heme $b$ subunit intact. Hitherto, one mitochondrial SC has evaded detection in prokaryotes: dimeric ATP synthase and its-string-like multimeric forms. This should not be a surprise, considering that bacteria do not have cristae. However, we did find dimeric and multimeric ATP synthase in the Archaeon M. thermautotrophicus under certain growth conditions (Figs. 11B-C) (Farhoud et al., 2005), and it cannot be ruled out a priori that such dimers and multimers could be found in other prokaryotes, especially when equipped with convolute laminar, tubular or highly

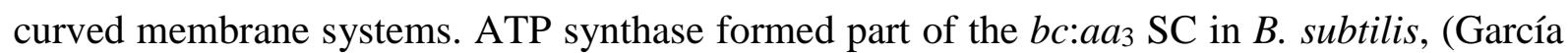
Montes de Oca et al., 2012). For Bacillus pseudofirmus OF4 a SC consisting of cytochrome $a a_{3}$ and ATP synthase has been described (Liu et al., 2007). Such an assembly could be physiologically essential. B. pseudofirmus is an alkaliphilic bacterium and an alkaline environment opposes the building up of a pmf. The association between cytochrome $a a_{3}$ that pumps protons out the cell and ATP synthase that is driven by the import of protons brings proton exchange in immediate proximity.

The above findings present firm indications that SC formation is relevant in microbial respiration, although their function is not completely clear, neither for bacterial, nor mitochondrial SCs. However, the eventual picture may be more diffuse and complex. By labelling respiratory RMPs with green fluorescent protein while maintaining activity after expression, confocal laser microscopy applied to living cells of B. subtilis and E. coli showed that RMPs are not evenly distributed across the cell membrane (Johnson et al., 2004; Lenn et 
al., 2008; Erhardt et al., 2014). RMPs were accumulated in slowly moving small patches sizing roughly $300 \mathrm{~nm}$ in diameter. Single-molecule studies with E. coli on NDH-1, SDH, cytochrome $b d$ oxidase, cytochrome $c a a_{3}$ oxidase labelled with different fluorophores confirmed that all these RMPs were consistently confined to the patches, albeit in various amounts (NDH-1, 10-20 molecules; SDH, 20-40; cydAB, 70-180; cyt caa, $24-45$; ATP synthase, 40-60) (Llorente-Garcia et al., 2014; Lenn and Leake, 2015). In contrast, a fluorescent ubiquinone derivative was evenly distributed across the whole membrane. Within these patches, different RMPs moved at different rates, but they did not co-migrate, as far as limits in resolution allow such conclusion. Obviously, lack of co-migration strongly argues against SC formation. However, it should be noted that the fdo:cydAB and fdo:cyt $\mathrm{caa}_{3} \mathrm{SCs}$ observed by BNE (see above) were not considered specifically. It also cannot be excluded that fluorescent labelling prevented aggregation of RMPs. Yet, taking SCs for granted, regulation of respiratory activities may include one more level, membrane transertion/ segregation resulting in the local and polar distribution of respiratory complexes (Lenn et al., 2008; Fishov and Norris; Magalon and Alberge, 2015; Matsumoto et al., 2015).

\subsection{The interactome}

In order to become active, respiratory RMPs have to be assembled (Sargent, 2007; Price and Driessen, 2010; Luirink et al., 2012), which requires the precisely timed action of generic and enzyme-specific machineries that insert catalytic and electron transfer cofactors into the subunits or add, for structural purposes, other molecules to specific amino acids. Such an assembly involves the directed placement of these subunits inside or outside the membrane, $\mathrm{N}$ - and/or C-terminal cleavage events related with peptide translocation across the membrane, proper folding, subunit interactions or further maturing of enzyme complexes. Next, catalytic activity may be controlled by PTM, which requires an association, no matter how brief, with 
enzymes that add or remove these molecules. Also other associations with low-molecularweight compounds or proteins may affect structures of RMPs, resulting in the inhibition or stimulation of their activities. The sum total of all permanent or temporary interactions of an enzyme with other proteinaceous or non-peptide partners is called its interactome.

Hereafter we will give, on the basis of recent reviews, a bird's-eye-view of what is known about the assembly of bacterial RMPs, and how proteomics has contributed to this knowledge or may contribute to a better understanding in the near future. In addition, we will briefly deal with the toolboxes that proteomics provides to pinpoint the interactome of an RMP and to unravel the dynamics of such interaction networks.

\subsubsection{Assembly of bacterial respiratory complexes}

As alluded above, the assembly of RMPs involves the action of generic machineries that translocate polypeptides through the membrane. The common systems in bacteria are the Sec, YidC and TAT translocons. The Sec ("secretory”) system threads translated polypeptide chains in an unfolded form to the periplasm, which is associated with the cleavage of an Nterminal leader sequence by a specific peptidase (du Plessis et al., 2011; Paetzel, 2014; Sala et al., 2014). The Sec system is assisted by the YidC protein, which correctly inserts TMHs (Dalbey et al., 2014). In contrast, in the "twin-arginine translocation” (TAT) pathway proteins are taken across the lipid bilayer in a pre-assembled and pre-folded manner (Fröbel et al., 2012; Robinson et al., 2012; Goosens et al., 2013, 2014a,b; Hopkins et al., 2014; Cline, 2015). The pathway is named after two adjacent arginines that typically are located about 20 positions after the translation start. Pre-assembly within the cytoplasm may include the insertion of iron-sulphur clusters synthesised by the Suf and ISC systems (Blanc et al., 2015; Wayne Outten, 2015; Kim et al., 2015; Yokoyama and Leimkühler, 2015) or of the molybdopterin cofactor, which is the catalytic heart of a wide variety of respiratory enzymes, 
including formate dehydrogenase and nitrate reductase (Grimaldi et al., 2013). Translation at the ribosomes and transport through the membrane can be intimately coupled by action of the Signal Recognition particle and Trigger Factor and of the DnaK and GroeEL protein folding apparatus (Castanié-Cornet et al., 2014; Saraogi and Shan, 2014). In case of molybdopterin proteins, members of the NarJ family perform a proofreading to check for correct folding before the peptide is directed to the TAT system (Lanciano et al., 2007; Chan et al., 2014; Bay et al., 2015). Haems that act in electron transfer, such as $c$ - and $b$-type haems, or in catalysis by terminal oxidases and nitric oxide reductases ( $b$-, $a$ - and $o$-type haems) are localised at the outer aspect of the membranes. So, before incorporation into a subunit(s), these molecules have to be translocated as well, which is realised by the cytochrome $c$ maturation (CCM) machinery (Kranz et al., 2009; Verissimo and Dalda, 2014). Periplasmic subunits can be attached to the membrane by covalently bound lipid molecules. This covalent binding is realised in the lipoprotein modification pathway (Hutchings et al., 2009; Buddelmeijer, 2015). Besides the prosthetic groups mentioned, RMPs depend on specific catalytic metals $(\mathrm{Cu}, \mathrm{Fe})$. To acquire these metals, usually at the periplasm, and insert them into the subunits, enzyme-specific chaperones are at the disposal (Robinson, 2010; Hanappel et al., 2012; Abicht et al., 2014; Dash et al., 2015). All in all, enzyme maturation is highly complex, wherein each RMP depends on its dedicated set of assembly factors acting in concert. Assembly processes of the major RMPs, including quinol:cytochrome $c$ oxidoreductases (Smith et al., 2012; Hasan et al., 2013, 2014; ), terminal oxidases ( Bühler et al., 2010; Ekici et al., 2012; Gurumoorthy et al., 2015), nitrite-/ nitric oxide-/ nitrous oxide reductases (Hatzixanthis et al., 2005; Barth et al., 2009; Spiro, 2012; Nicke et al., 2013; Adamczack et al., 2014), hydrogenases (Peters et al., 2015), formate dehydrogenases (Hartmann et al., 2015) and other molybdopterin-containing RMPs (Arnoux et al., 2015; Magalon and Mendel, 2015) have been studied in more or less detail. Notoriously, the 
assembly of complex I with its about 45 subunits in unicellular and higher eukaryotes is puzzling (Vogel et al., 2007c; Mimaki et al., 2014; Vartak et al., 2014). Assembly should be much simpler for prokaryotic NDHs that harbour a mere 13 subunits, but virtually nothing is known about it. Most of our knowledge on RMP assemblages has been gained from tedious genetic and biochemical studies, but this knowledge is often still fragmentary and far from complete.

Hitherto, relatively few studies that were aimed at unravelling assembly pathways of RMPs have taken full advantage of the potentials offered by proteomics. Most of these studies applied proteomics methods to detect the effect of gene deletion, making differential expression analyses by 2DGE or shotgun methods outlined in Section 3.3. The majority of the latter studies were directed at membrane transport processes of polypeptides, more specifically at the role of Sec components (Baars et al., 2006, 2008), YidC protein (Wickström et al., 2011b), Trigger Factor (Wickström et al., 2011a), TAT proteins (Hitchcock et al., 2010) and of periplasmic chaperones (Götzke et al., 2015) in these processes. In a broader context, established and new proteomics methods were used to make inventories of secreted proteins ("secretome") by mapping polypeptides that were transported across the cytoplasmic membrane or beyond (see f.i. Zijnge et al., 2012; Johnson et al., 2013; Renier et al., 2015). These maps supported the evaluation of signal peptide algorithms (Leversen et al., 2009) and even suggested the presence of yet-unknown peptide transport systems (Wilson et al., 2015).

To identify binding partners of chaperones that assist in the insertion of copper into the catalytic subunits of cytochrome $a a_{3}$ oxidase from $P$. denitrificans, Gurumoorthy and colleagues (2015) used these chaperones as baits in pull-down and crosslink experiments. Wickström et al. (2011b) employed BNE (Section 3.2.2.) to assess folding intermediates and partially assembled RMPs that accumulated in the cytoplasm as the result of YidC depletion. In order to start understanding how NDH (NuoA-N) is assembled in prokaryotes, Erhardt et al. 
(2012) also applied BNE. The authors explored the effect of the disruption of various nuo genes by analysing partially assembled NDH complexes.

Indeed, BNE may turn out to become a powerful instrument to elucidate the assembly of RMPs (see for OXPHOS complexes: McKenzie et al., 2007; Calvaruso et al., 2008; Gil Borlado et al., 2010; Leary, 2012), especially by complexome profiling (Section 3.2.2; Fig. 12). Here, RMPs from cells grown under different conditions and/ or isolated from the membranes by different detergents are separated by Blue Native gel electrophoresis and comigrating polypeptides are profiled using recently developed software (Giese et al., 2015). This analysis may establish already known, fully or incompletely assembled RMPs, but also may reveal new RMPs. Next, known or yet unknown co-migrating partners, i.e. other than subunits, may be found, opening a way for further exploration of their function. The potential of this BNE profiling method is exemplified by several studies on mitochondrial OXPHOS complexes, which enabled the identification of new assembly factors (Wessels et al., 2009, 2013; Heide et al. (2012),

\subsubsection{The far from complete picture of interaction partners and interaction dynamics}

Proteomics offers the means to investigate the interaction of proteins on all organisational levels and on different timescales, ranging between stable subunit associations, temporary assembly intermediates, and contacts with assembly factors, enzymes involved in PTMs or other high- or low-molecular-weight compounds that affect activity. In fact, all available methods have been described in previous sections. Here we will only pinpoint from the global and enzyme-directed perspectives, how these methods can be employed to unravel interactome dynamics.

The method of choice for a global analysis of protein interactions is BNE (see Section 3.2.2 and the previous Section 5.2.1) (Krause, 2006; Heide and Wittig, 2013). Even though 
such global analysis provides a wealth of data, this data may lack detail, but BNE is applicable in the study of individual complexes as well. Actually, most MS-based approaches take isolated enzymes as the starting point to explore their interactomes (Gingras et al., 2007; Wepf et al., 2009; Dengjel et al., 2010; Ramisetty and Washburn, 2011). Proteins are generally purified in their tagged forms and partners that co-elute from affinity columns are rigorously screened for physiologically relevance (see Section 4.1.1) (Vogel et el. 2007a,b; Saada et al., 2009; Nouws et al., 2010; van den Ecker et al., 2010; Tucker et al., 2013; Formosa et al., 2015). In principle, this screening can even be done by high-throughput robotics (Hakhverdyan et al., 2015). Time series at which proteins become labelled in vivo (SILAC) or at which isolated protein samples are labelled by in vitro methods (see Section 2.3) may detect temporary interactions in multiplexed tandem MS analyses (Ramisetty and Washburn, 2011). These interactions can be "frozen” by in vivo or in vitro crosslinking (Tran et al., 2015). The detailed inspection of crosslinked peptides permits the pinpointing of contact sides between the different proteins. In H/D exchange experiments or by probing proteins by hydroxyl radicals or other reagents (see Section 4.1.3), conformational dynamics due to protein-protein interactions or induced by PTMs, ligand or metal-ion bindings may be unravelled (Ramisetty and Washburn, 2011; Venable et al., 2015). Whereas the above approaches are shotgun-MS oriented, novel mass spectrometry methods allow interaction studies to be made on whole protein complexes and other associates (see Section 4.1.5). Like in many other occasions, only little use has been made of MS-based methods in research on RMP interactomes. Experimental challenges posed by membrane-bound proteins and inaccessibility to the constantly developing field of proteomics may be reasons for this.

\section{PERSPECTIVES}


Within the wide field of proteomics, an area of methods and techniques has been established to study protein chemistry in unprecedented detail and accuracy. Proteomics offers the means to make an inventory of all expressed proteins in all their proteoforms in an organism and to quantitatively map changes in this inventory that are made by the organism in response to changes in its environment. From the perspective of the isolated enzyme (system), proteomics enables the collection of information on protein structures and dynamics, lending the field a central place in structural biology.

By now, over $80 \%$ of all proteins that are coded by a bacterial genome can be detected on a high-throughput basis, if whole cell preparations are analysed by appropriate and complementary pre-fractionation methods (Section 3.1) (Catrein and Herrmann., 2011; Soares et al., 2013; Soufi et al., 2015). This percentage may even be an underestimation, since not all proteins are expressed under the investigated growth conditions. Also membrane-bound proteins that had been a real nuisance for a long time can be identified with a high success rate (Section 3.2.1). Routine methods are available to identify and quantify PTMs (phosphorylation, acetylation, methylation, oxidative stress-induced structural changes) in protein sequences at large scale (Section 4.2). By label-free or in vivo or in vitro labelling techniques (Section 2.3), differential expression can be profiled quantitatively in multiplexed experiments (Section 3.3), providing invaluable data for systems-biology endeavours (see f.i. Wegener et al., 2010; Kohlmann et al., 2011, 2014; Hui et al., 2015; Soufi et al., 2015).

The above outcome primarily relies on shotgun proteomics. Due to its success it seems that classical gel-based proteomics to have taken a place on the back seat. Still, this secondrank position may be unjustified (Oliveira et al., 2014). As pointed out, the strengths of 2DE are the visibility of proteins (Figs. 9, 11, 13) and its relatively easy access by non-experts (Sections 3.1.1, 3.2.2 and 3.3). On the other hand, the disadvantages of 2DE are its insensitivity and, if not robotised, the workload that is associated with the identification of gel 
spots: each protein spot has to be extracted from the gel and analysed by MS individually. Despite these obvious shortcomings, gel-based methods still have their undeniable advantages as exemplified by BNE and CNE (Section 3.2.2). By their assistance in the elucidation of respiratory supercomplexes, BNE and CNE even caused a paradigm shift in our understanding of electron transport chains (Section 5.1). The stronghold of these methods is that enzyme systems, also in very complex protein mixtures, are investigated as their native complexes, even associated with other interaction partners like assembly factors (Section 5.2). This information is lost in classical shotgun approaches. BNE and CNE combined with complexome profiling allow the detection of completely new RMPs and their subunit composition (Section 3.2.2; Fig. 12). In this respect, BNE and CNE may become powerful tools in eliciting new respiratory systems in poorly studied microorganisms that come to us at an incredible pace. Here, gel-based and shotgun proteomics may go hand in hand by clever combinations of top-down and bottom-up approaches to get the maximum structural information out of minimal amounts of proteins (Strader et al., 2004; Millea et al. 2006; Wu et al., 2009).

Indeed, a definite advantage of proteomics is that only minimal amounts of proteins are required for structural studies. Miniaturised liquid column chromatography, affinitybinding and other methods are available or are being developed for the targeted purification of enzymes in their native form (Section 4.1.1). Purified preparations can be analysed on-line or off-line by MS. In sections 4.1.3 (Fig. 15) and 4.1.5, we presented an overview of shotgunbased techniques that inform us on secondary, tertiary and quaternary aspects of protein complexes, and of the progress that has been made in studying whole complexes and their dynamics from the top-down perspective. It was stated (Section 4.1.6) that the combined information obtained from proteomics experiments would allow the proposal of 3D structures, although they currently can achieve only a modest resolution ( $\sim \AA$ ). This could be highly 
relevant in the case of RMPs that are notoriously difficult to investigate by X-ray crystallography and NMR spectroscopy.

Finishing this review, the reader may have wondered that its topic, bacterial electron transfer systems, may have been underexposed. This is definitely true, but it reflects the present status of the application of proteomics to understand bacterial ETS. Nevertheless, we hope that we may have given an impression of how proteomics may contribute to come to a full understanding of these systems in all their variations that emerged during evolution.

\section{ACKNOWLEDGEMENTS}

The authors would like to thank Dr. Alain van Gool, Dr. Dirk Lefeber, Dr. Monique van Scherpenzeel and Ms. Esther Willems for their contribution in generating the glycopeptide tandem mass spectra shown in Figure 4. This work was supported by the Netherlands Organization for Scientific Research (NWO) by ALW grant ALW2PJ/08021 to Naomi de Almeida and VENI grant 863.11.003 to Boran Kartal. 


\section{REFERENCES}

Abicht, H.K., Schärer, M.A., Quade, N., Ledermann, R., Mohorko, E., Capitani, G., Hennecke, H. and Glockshuber, R. (2014). How periplasmic thioredoxin TlpA reduces bacterial copper chaperone ScoI and cytochrome oxidase subunit II (CoxB) prior to metallation. J. Biol. Chem. 289, 324313244.

Acín-Pérez R., Fernández-Silva, P., Peleato, M.L., Pérez-Martos, A. and Enriquez, J.A. (2008) Respiratory active mitochondrial supercomplexes. Mol. Cell. 21, 529-539.

Acin-Perez, R. and Enriquez, J.A. (2014). The function of the respiratory supercomplexes: the plasticity model. Biochim. Biophys. Acta 1837, 444-450.

Adamczack, J., Hoffmann, M., Papke, U., Haufschildt, K., Nicke, T., Bröring, M., Sezer, M., Weimar, R., Kuhlmann, U., Hildebrandt, P. and Layer, G. (2014). NirN protein from Pseudomonas aeruginosa is a novel electron-bifurcating dehydrogenase catalyzing the last step of heme $\mathrm{d} 1$ biosynthesis. J. Biol. Chem. 289, 30753-30762.

Aebersold, R. and Mann, M. (2003). Mass spectrometry-based proteomics. Nature 422, 198-207.

Aggarwal, S. and Yadav, A. K. (2016). False Discovery Rate Estimation in Proteomics. Methods Mol. Biol. 1362, 119-128.

Agrawal, G.K. and Thelen, J.J. (2005) Development of a simplified, economical polyacrylamide gel staining protocol for phosphoproteins. Proteomics 5, 4684-4688.

Aivaliotis, M., Macek, B., Gnad, F., Reichelt, P., Mann, M. and Oesterhelt, D. (2009). Ser/Thr/Tyr protein phosphorylation in the archaeon Halobacterium salinarum-a representative of the third domain of life. PLoS One 4:e4777.

Al-Attar, S. and de Vries, S. (2013). Energy transduction by respiratory metallo-enzymes: from molecular mechanism to cell physiology. Coord. Chem. Rev. 257, 64-80.

Alber T. (2009). Signaling mechanisms of the Mycobacterium tuberculosis receptor Ser/Thr protein kinases. Curr. Opin. Struct. Biol. 19, 650-657.

Alberge, F., Espinosa, L., Seduk, F., Sylvi, L., Toci, R., Walburger, A. and Magalon A. (2015). Dynamic subcellular localization of a respiratory complex controls bacterial respiration. Elife 16:4.

Allison, T.M., Reading, E., Liko, I., Baldwin, A.J., Laganowsky, A., Robinson, CV. (2015) Quantifying the stabilizing effects of protein-ligand interactions in the gas phase. Nat. Commun. 6:8551.

Allmer, J. (2011). Algorithms for the de novo sequencing of peptides from tandem mass spectra. Expert Rev. Proteomics 8, 645-657.

Andaya, A., Villa, N., Jia, W., Fraser, C.S. and Leary J.A. (2014). Phosphorylation stoichiometries of human eukaryotic initiation factors. Int. J. Mol. Sci. 15, 11523-11538.

Anderson, K.A., Hirschey and M.D. (2012). Mitochondrial protein acetylation regulates metabolism. Essays Biochem. 52, 23-35.

Ansong, C., Wu, S., Meng, D., Liu, X., Brewer, H.M., Deatherage Kaiser B,L., Nakayasu E.S., Cort, J.R., Pevzner, P., Smith, R.D., Heffron, F., Adkins, J.N and Pasa-Tolic, L. (2013). Top-down proteomics reveals a unique protein S-thiolation switch in Salmonella typhimurium in response to infection-like conditions. Proc. Natl. Acad. Sci. U S A 110, 10153-10158.

Antelmann, H. and Helmann J.D. (2011). Thiol-based redox switches and gene regulation. Antioxid. Redox Signal. 14, 1049-1063.

Arias-Cartin, R., Grimaldi, S., Arnoux, P., Guigliarelli, B. and Magalon, A. (2012). Cardiolipin binding in bacterial respiratory complexes: structural and functional implications. Biochim. Biophys. Acta 1817, 1937-1949.

Arias-Cartin, R/, Grimaldi. S/, Pommier. J/, Lanciano. P/, Schaefer. C/, Arnoux. P/, Giordano. G., Guigliarelli, B. and Magalon, A. (2011) Cardiolipin-based respiratory complex activation in bacteria. Proc. Natl. Acad. Sci. U S A. 108, 7781-7786.

Arnoux, P., Ruppelt, C., Oudouhou, F., Lavergne, J., Siponen, M.I., Toci, R., Mendel, R.R., Bittner, F., Pignol, D., Magalon, A. and Walburger A. (2015) Sulphur shuttling across a chaperone during molybdenum cofactor maturation. Nat. Commun. 6:6148.

Baars, L., Ytterberg, A.J., Drew, D., Wagner, S., Thilo, C., van Wijk, K.J. and de Gier J.W. (2006). Defining the role of the Escherichia coli chaperone SecB using comparative proteomics. J. Biol. Chem. 281, 10024-10034. 
Baars, L., Wagner, S., Wickström, D., Klepsch, M., Ytterberg, A.J., van Wijk, K.J. and de Gier J.W. (2008). Effects of SecE depletion on the inner and outer membrane proteomes of Escherichia coli. J. Bacteriol. 190, 3505-3525.

Babot, M., Birch, A., Labarbuta, P. and Galkin A. (2014). Characterisation of the active/de-active transition of mitochondrial complex I. Biochim. Biophys. Acta 1837, 1083-1092.

Bache, N., Rand, K.D., Roepstorff, P. and Jørgensen, T.J. (2008). Gas-phase fragmentation of peptides by MALDI in-source decay with limited amide hydrogen $(1 \mathrm{H} / 2 \mathrm{H})$ scrambling. Anal. Chem. 80, 6431-6435.

Banks, C.A. and Kong, S.E. and Washburn, M.P. (2012). Affinity purification of protein complexes for analysis by multidimensional protein identification technology. Protein Expr. Purif. 86, 105-119.

Barber, M., Bordoli, R. S., Sedgwick, R. D. and Tyler, A. N. (1981). Fast atom bombardment of solids (F.A.B.): a new ion source for mass spectrometry. J. Chem. Soc., Chemical Communications, 325327.

Barrera, N.P., Di Bartolo, N., Booth, P.J. and Robinson, CV. (2008). Micelles protect membrane complexes from solution to vacuum. Science 321, 243-246.

Barrera, N.P., Zhou, M. and Robinson, C.V. (2013). The role of lipids in defining membrane protein interactions: insights from mass spectrometry. Trends Cell. Biol. 23, 1-8.

Barth, K.R., Isabella, V.M. and Clark, V.L. (2009). Biochemical and genomic analysis of the denitrification pathway within the genus Neisseria. Microbiology 155, 4093-4103.

Bäsell, K., Otto, A., Junker, S., Zühlke, D., Rappen, G.M., Schmidt, S., Hentschker, C., Macek, B., Ohlsen, K., Hecker, M. and Becher, D. (2014). The phosphoproteome and its physiological dynamics in Staphylococcus aureus. Int. J. Med. Microbiol. 304, 121-132.

Batista, A.P., Franco, C., Mendes, M., Coelho, A.V. and Pereira, M.M. (2010). Subunit composition of Rhodothermus marinus respiratory complex I. Anal. Biochem. 407, 104-110.

Battchikova, N. and Aro, E.M. (2007). Cyanobacterial NDH-1 complexes: multiplicity in function and subunit composition. Physiol. Plant. 131, 22-32.

Bay, D.C., Chan, C.S., Turner and R.J. (2015). NarJ subfamily system specific chaperone diversity and evolution is directed by respiratory enzyme associations. BMC Evol. Biol. 15:110.

Bazán. S., Mileykovskaya, E., Mallampalli, V.K., Heacock. P., Sparagna. G.C. and Dowhan, W. (2013). Cardiolipin-dependent reconstitution of respiratory supercomplexes from purified Saccharomyces cerevisiae complexes III and IV. J. Biol. Chem. 288, 401-411.

Becher, D., Büttner, K., Moche, M., Hessling, B. and Hecker M. (2011). From the genome sequence to the protein inventory of Bacillus subtilis. Proteomics 11, 2971-2980.

Benesch, J.L. and Robinson, C.V. (2006). Mass spectrometry of macromolecular assemblies: preservation and dissociation. Curr Opin. Struct. Biol. 16, 245-251.

Benesch, J.L., Aquilina, J.A., Ruotolo, B.T., Sobott, F. and Robinson, C.V. (2006). Tandem mass spectrometry reveals the quaternary organization of macromolecular assemblies. Chem. Biol. 13, 597-605.

Bennett, K.L., Matthiesen, T. and Roepstorff, P. (2000). Probing protein surface topology by chemical surface labeling, crosslinking, and mass spectrometry. Methods Mol. Biol. 146, 113-131.

Bereman, M.S., MacLean, B., Tomazela, D. M., Liebler, D.C. and MacCoss, M.J. (2012). The development of selected reaction monitoring methods for targeted proteomics via empirical refinement. Proteomics 12, 1134-1141.

Bernal, V., Castaño-Cerezo, S., Gallego-Jara, J., Écija-Conesa, A., de Diego, T., Iborra, J.L. and Cánovas, M. (2014). Regulation of bacterial physiology by lysine acetylation of proteins. Nat. Biotechnol. 31, 586-595.

Bernard-Marty, C., Lebrun, F., Awada, A. and Piccart, M. J. (2006). Monoclonal antibody-based targeted therapy in breast cancer: current status and future directions. Drugs 66, 1577-1591.

Bernsel, A. and Daley, D.O. (2009). Exploring the inner membrane proteome of Escherichia coli: which proteins are eluding detection and why? Trends Microbiol. 17, 444-449.

Berven, F.S., Karlsen, O.A., Murrell, J.C. and Jensen, H.B. (2003). Multiple polypeptide forms observed in two-dimensional gels of Methylococcus capsulatus (Bath) polypeptides are generated during the separation procedure. Electrophoresis 24, 757-761.

Biemann, K. (1990). Appendix 5. Nomenclature for peptide fragment ions (positive ions). Methods Enzymol. 193, 886-887. 
Bisle, B., Schmidt, A., Scheibe, B., Klein, C., Tebbe, A., Kellermann, J., Siedler, F., Pfeiffer, F., Lottspeich, F. and Oesterhelt, D. (2006). Quantitative profiling of the membrane proteome in a halophilic archaeon. Mol. Cell. Proteomics 5, 1543-1558.

Blanc, B., Gerez, C. and Ollagnier de Choudens, S. (2015). Assembly of Fe/S proteins in bacterial systems: Biochemistry of the bacterial ISC system. Biochim. Biophys. Acta 1853, 1436-1447.

Blaza, J.N., Serreli, R., Jones, A.J., Mohammed, K. and Hirst, J. (2014). Kinetic evidence against partitioning of the ubiquinone pool and the catalytic relevance of respiratory-chain supercomplexes. Proc. Natl.Acad. Sci. U S A. 111, 15735-15740.

Bleier, L., Wittig, I., Heide, H., Steger, M., Brandt, U. and Dröse, S. (2015). Generator-specific targets of mitochondrial reactive oxygen species. Free Radic. Biol. Med. 78, 1-10.

Block, H., Maertens, B., Spriestersbach, A., Brinker, N., Kubicek, J., Fabis, R., Labahn, J. and Schäfer, F. (2009). Immobilized-metal affinity chromatography (IMAC): a review. Methods Enzymol. 463, 439-473.

Boersema, P.J., Aye, T.T., van Veen, T.A., Heck, A.J. and Mohammed, S. (2008). Triplex protein quantification based on stable isotope labeling by peptide dimethylation applied to cell and tissue lysates. Proteomics 8, 4624-4632.

Boersema, P.J., Raijmakers, R., Lemeer, S., Mohammed, S. and Heck, A.J. (2009). Multiplex peptide stable isotope dimethyl labeling for quantitative proteomics. Nat. Protoc. 4, 484-494.

Boersema, P.J., Kahraman, A. and Picotti, P. (2015). Proteomics beyond large-scale protein expression analysis. Curr. Opin. Biotechnol. 34, 162-170.

Bohovych, I., Fernandez, M.R., Rahn, J.J., Stackley, K.D., Bestman, J.E., Anandhan, A., Franco, R., Claypool, S.M., Lewis, R.E., Chan, S.S. and Khalimonchuk, O. (2015). Metalloprotease OMA1 Fine-tunes Mitochondrial Bioenergetic Function and Respiratory Supercomplex Stability. Sci Rep. 5:13989.

Borisov, V.B., Gennis, R.B., Hemp, J. and Verkhovsky, M.I. (2011). The cytochrome bd respiratory oxygen reductases. Biochim. Biophys. Acta 1807, 1398-1413.

Bornhövd, C., Vogel, F., Neupert, W. and Reichert, A.S. (2006). Mitochondrial membrane potential is dependent on the oligomeric state of F1F0-ATP synthase supracomplexes. J. Biol. Chem. 281, 13990-13998.

Borysik, A.J., Hewitt, D.J and Robinson, C.V. (2013). Detergent release prolongs the lifetime of nativelike membrane protein conformations in the gas-phase. J. Am. Chem. Soc. 135, 6078-6083.

Brancia, F.L. (2006). Recent developments in ion-trap mass spectrometry and related technologies. Expert Rev. Proteomics 3, 143-151.

Brandt, U. (2011). A two-state stabilization-change mechanism for proton-pumping complex I. Biochim. Biophys. Acta 1807, 1364-1369.

Braun, R.J., Kinkl, N., Beer, M. and Ueffing, M. (2007). Two-dimensional electrophoresis of membrane proteins. Anal. Bioanal. Chem. 389, 1033-1045.

Brewis, I.A. and Brennan, P. (2010). Proteomics technologies for the global identification and quantification of proteins. Adv. Protein. Chem. Struct. Biol. 80, 1-44.

Bridges, H.R., Fearnley, I.M. and Hirst, J. (2010). The subunit composition of mitochondrial NADH:ubiquinone oxidoreductase (complex I) from Pichia pastoris. Mol. Cell. Proteomics 9, 2318-2326.

Brosch, M., Yu, L., Hubbard, T. and Choudhary, J. (2009). Accurate and sensitive peptide identification with Mascot Percolator. J. Proteome Res. 8, 3176-3181.

Brown, C.T., Hug, L.A., Thomas, B.C., Sharon, I., Castelle, C.J., Singh, A., Wilkins, M.J., Wrighton, K.C., Williams, K.H. and Banfield, J.F. (2015). Unusual biology across a group comprising more than 15\% of domain Bacteria. Nature 523, 208-211.

Brown, R.N., Romine, M.F., Schepmoes, A.A., Smith, R.D. and Lipton, M.S. (2010). Mapping the subcellular proteome of Shewanella oneidensis MR-1 using sarkosyl-based fractionation and LCMS/MS protein identification. J. Proteome Res. 9, 4454-4463.

Brown, R.S. and Lennon, J.J. (1995). Mass resolution improvement by incorporation of pulsed ion extraction in a matrix-assisted laser desorption/ionization linear time-of-flight mass spectrometer. Anal. Chem. 67, 1998-2003.

Buddelmeijer, N. (2015). The molecular mechanism of bacterial lipoprotein modification--how, when and why? FEMS Microbiol. Rev. 39, 246-261. 
Bühler, D., Rossmann R., Landolt, S., Balsiger, S., Fischer, H.M. and Hennecke, H. (2010). Disparate pathways for the biogenesis of cytochrome oxidases in Bradyrhizobium japonicum. J. Biol. Chem. 285, 15704-15713.

Bultema, J.B., Braun, H.P., Boekema, E.J. and Kouril, R. (2009). Megacomplex organization of the oxidative phosphorylation system by structural analysis of respiratory supercomplexes from potato. Biochim. Biophys. Acta 1787, 60-67.

Bunai, K. and Yamane, K. (2005). Effectiveness and limitation of two-dimensional gel electrophoresis in bacterial membrane protein proteomics and perspectives. J. Chromatogr. B Analyt. Technol. Biomed. Life Sci. 815, 227-236.

Burns, K.M., Sarpe, V., Wagenbach, M., Wordeman, L. and Schriemer, D.C. (2015). HX-MS² for high performance conformational analysis of complex protein states. Protein Sci. 24, 1313-1324.

Calabrese, A.N., Watkinson, T.G., Henderson PJ, Radford SE, Ashcroft AE. (2015). Amphipols outperform dodecylmaltoside micelles in stabilizing membrane protein structure in the gas phase. Anal. Chem. 87, 1118-1126.

Calder, B., Soares, N.C., de Kock, E. and Blackburn, J.M. (2015). Mycobacterial proteomics: analysis of expressed proteomes and post-translational modifications to identify candidate virulence factors. Expert. Rev. Proteomics. 12, 21-35.

Calvaruso, M.A., Smeitink, J. and Nijtmans, L. (2008). Electrophoresis techniques to investigate defects in oxidative phosphorylation. Methods 46, 281-287.

Calvaruso, M.A., Willems, P., van den Brand, M., Valsecchi, F., Kruse, S., Palmiter, R., Smeitink, J. and Nijtmans, L. (2012). Mitochondrial complex III stabilizes complex I in the absence of NDUFS4 to provide partial activity. Hum. Mol. Genet. 21, 115-120.

Carlson, S.M., Moore, K.E., Green, E.M., Martín, G.M. and Gozani, O. (2014). Proteome-wide enrichment of proteins modified by lysine methylation. Nat. Protoc. 9, 37-50.

Caro-Quintero, A. and Konstantinidis, K.T. (2012). Bacterial species may exist, metagenomics reveal. Environ. Microbiol. 14, 347-355.

Carroll, J., Fearnley, I.M., Shannon, R.J., Hirst, J. and Walker, J.E. (2003).Analysis of the subunit composition of complex I from bovine heart mitochondria. Mol. Cell. Proteomics. 2, 117-126.

Carroll, J., Ding, S., Fearnley, I.M. and Walker, J.E. (2013). Post-translational modifications near the quinone binding site of mammalian complex I. J. Biol. Chem. 288, 24799-24808.

Cartron, M.L., Olsen, J.D., Sener, M., Jackson, P.J., Brindley, A.A., Qian, P., Dickman, M.J., Leggett, G.J., Schulten, K. and Neil Hunter, C. (2014). Integration of energy and electron transfer processes in the photosynthetic membrane of Rhodobacter sphaeroides. Biochim. Biophys. Acta 1837, 17691780.

Castanié-Cornet, M.P., Bruel, N., Genevaux, P. (2014). Chaperone networking facilitates protein targeting to the bacterial cytoplasmic membrane. Biochim. Biophys. Acta 1843, 1442-1456.

Castelle, C., Guiral, M., Malarte, G., Ledgham, F., Leroy, G., Brugna, M. and Giudici-Orticoni, M.T. (2008). A new iron-oxidizing/ $\mathrm{O}_{2}$-reducing supercomplex spanning both inner and outer membranes, isolated from the extreme acidophile Acidithiobacillus ferrooxidans. J. Biol. Chem. 283, 25803-25811.

Castelle, C.J., Roger, M., Bauzan, M., Brugna, M., Lignon, S., Nimtz, M., Golyshina, OV, GiudiciOrticoni, M.T. and Guiral, M. (2015). The aerobic respiratory chain of the acidophilic archaeon Ferroplasma acidiphilum: A membrane-bound complex oxidizing ferrous iron. Biochim. Biophys. Acta 1847, 717-728.

Catherman, A.D., Li, M., Tran, J.C., Durbin, K.R., Compton, P.D., Early, B.P., Thomas, P.M. and Kelleher N.L. (2013). Top down proteomics of human membrane proteins from enriched mitochondrial fractions. Anal. Chem. 85, 1880-1888.

Catherman, A.D., Skinner, O.S. and Kelleher, N.L. (2014). Top Down proteomics: facts and perspectives. Biochem. Biophys. Res. Commun. 445, 683-693.

Catrein, I. and Herrmann, R. (2011). The proteome of Mycoplasma pneumoniae, a supposedly "simple" cell. Proteomics 11, 3614-3632.

Černý, M., Skalák, J., Cerna, H. and Brzobohatý, B. (2013). Advances in purification and separation of posttranslationally modified proteins. J. Proteomics. 92, 2-27. 
Chaban, Y., Boekema, E.J. and Dudkina, N.V. (2014). Structures of mitochondrial oxidative phosphorylation supercomplexes and mechanisms for their stabilisation. Biochim. Biophys. Acta 1837, 418-426.

Chan, C.S., Bay, D.C., Leach, T.G., Winstone, T.M., Kuzniatsova, L., Tran, V.A. and Turner, R.J. (2014). 'Come into the fold': A comparative analysis of bacterial redox enzyme maturation protein members of the NarJ subfamily. Biochim. Biophys. Acta 1838, 2971-2984.

Chang, L., Liu, X., Li, Y., Liu, C.C., Yang, F., Zhao, J. and Sui, S.F. (2015). Structural organization of an intact phycobilisome and its association with photosystem II. Cell. Res. 25, 726-737.

Chapman, J.D., Goodlett, D. R. and Masselon, C.D. (2014). Multiplexed and data-independent tandem mass spectrometry for global proteome profiling. Mass Spectrom. Rev. 33, 452-470.

Chernushevich, I.V., Loboda, A.V. and Thomson, B.A. (2001). An introduction to quadrupole-time-offlight mass spectrometry. J. Mass Spectrom. 36, 849-865.

Cheung, R.C., Wong, J.H. and Ng, T.B.(2012). Immobilized metal ion affinity chromatography: a review on its applications. Appl. Microbiol. Biotechnol. 96, 1411-1420.

Chevalier, F. (2010). Highlights on the capacities of "Gel-based" proteomics. Proteome Sci. 8:23.

Chi, B.K., Gronau, K., Mäder, U., Hessling, B., Becher, D. and Antelmann, H. (2011). S-bacillithiolation protects against hypochlorite stress in Bacillus subtilis as revealed by transcriptomics and redox proteomics. Mol. Cell. Proteomics 10:M111.009506.

Chi, B.K., Roberts, A.A., Huyen, T.T., Bäsell, K., Becher, D., Albrecht, D., Hamilton, C.J. and Antelmann, H. (2013). S-bacillithiolation protects conserved and essential proteins against hypochlorite stress in firmicutes bacteria. Antioxid. Redox Signal. 18, 1273-1295.

Chi, B.K., Busche, T., Van Laer, K., Bäsell, K., Becher, D., Clermont, L., Seibold, G.M., Persicke, M., Kalinowski, J., Messens, J. and Antelmann, H. (2014). Protein S-mycothiolation functions as redox-switch and thiol protection mechanism in Corynebacterium glutamicum under hypochlorite stress. Antioxid. Redox Signal. 20, 589-605.

Chi, H., Chen, H., He, K., Wu, L., Yang, B., Sun, R.X., Liu, J., Zeng, W. F., Song, C.Q., He, S.M. and Dong, M.Q. (2013). pNovo+: de novo peptide sequencing using complementary HCD and ETD tandem mass spectra. J. Proteome Res. 12, 615-625.

Chiang, S.M. and Schellhorn, H.E. (2012). Regulators of oxidative stress response genes in Escherichia coli and their functional conservation in bacteria. Arch. Biochem. Biophys. 525, 161-169.

Choi, C.W., Yun, S.H., Kwon, S.O., Leem, S.H., Choi, J.S., Yun, C.Y. and Kim, S.I. (2010). Analysis of cytoplasmic membrane proteome of Streptococcus pneumoniae by shotgun proteomic approach. J. Microbiol. 48, 872-876.

Chojnacka, M., Gornicka, A., Oeljeklaus, S., Warscheid B., and Chacinska, A. (2015). Cox17 Protein Is an Auxiliary Factor Involved in the Control of the Mitochondrial Contact Site and Cristae Organizing System. J Biol Chem. 290, 15304-15312.

Chorev, D.S., Ben-Nissan, G. and Sharon, M. (2015). Exposing the subunit diversity and modularity of protein complexes by structural mass spectrometry approaches. Proteomics 15, 2777-2791.

Chouchani, E.T., Methner, C., Nadtochiy, S.M., Logan, A., Pell, V.R., Ding, S., James, A.M., Cochemé, H.M., Reinhold, J., Lilley, K.S., Partridge, L., Fearnley, I.M., Robinson, A.J., Hartley, R.C., Smith, R.A., Krieg, T., Brookes, P.S. and Murphy, M.P. (2013). Cardioprotection by S-nitrosation of a cysteine switch on mitochondrial complex I. Nat. Med. 19, 753-759.

Choudhary, C., Weinert, B.T., Nishida, Y., Verdin, E. and Mann, M. (2014). The growing landscape of lysine acetylation links metabolism and cell signalling. Nat. Rev. Mol. Cell. Biol. 15, 536-550.

Claypool, S.M. (2009). Cardiolipin, a critical determinant of mitochondrial carrier protein assembly and function. Biochim. Biophys. Acta 1788, 2059-2068.

Cline K. (2015). Mechanistic Aspects of Folded Protein Transport by the Twin Arginine Translocase (Tat). J. Biol. Chem. 290, 16530-16538.

Cogliati, S., Frezza, C., Soriano, M.E., Varanita, T., Quintana-Cabrera, R., Corrado, M., Cipolat, S., Costa, V., Casarin, A., Gomes, L.C., Perales-Clemente, E., Salviati, L., Fernandez-Silva, P., Enriquez, J.A. and Scorrano, L. (2013). Mitochondrial cristae shape determines respiratory chain supercomplexes assembly and respiratory efficiency. Cell 155, 160-171.

Colangelo, C.M., Chung, L., Bruce, C. and Cheung, K.H. (2013). Review of software tools for design and analysis of large scale MRM proteomic datasets. Methods 61, 287-298. 
Cologna, S.M., Russell, W.K., Lim, P.J., Vigh, G. and Russell, D.H. (2010). Combining isoelectric point-based fractionation, liquid chromatography and mass spectrometry to improve peptide detection and protein identification. J. Am. Soc. Mass Spectrom. 21, 1612-1619.

Cordwell, S.J. (2004). Exploring and exploiting bacterial proteomes. Methods Mol. Biol. 266, 115-135.

Cordwell, S.J., Len, A.C., Touma, R.G., Scott, N.E., Falconer, L., Jones, D., Connolly, A., Crossett, B. and Djordjevic, S.P. (2008). Identification of membrane-associated proteins from Campylobacter jejuni strains using complementary proteomics technologies. Proteomics 8, 122-139.

Cotter, R.J., Griffith, W. and Jelinek, C. (2007). Tandem time-of-flight (TOF/TOF) mass spectrometry and the curved-field reflectron. J. Chromatogr. B Analyt. Technol. Biomed. Life Sci. 855, 2-13.

Cousin, C., Derouiche, A., Shi, L., Pagot, Y., Poncet, S. and Mijakovic, I. (2013). Proteinserine/threonine/tyrosine kinases in bacterial signaling and regulation. FEMS Microbiol. Lett. 346, 11-19.

Cox, J. and Mann, M. (2008). MaxQuant enables high peptide identification rates, individualized p.p.b.range mass accuracies and proteome-wide protein quantification. Nat. Biotechnol. 26, 1367-1372.

Craig, R. and Beavis, R.C. (2004). TANDEM: matching proteins with tandem mass spectra. Bioinformatics 20, 1466-1467.

Croal, L.R., Gralnick, J.A., Malasarn, D., Newman, D.K. (2004). The genetics of geochemistry. Annu. Rev. Genet. 38, 175-202.

Crosby, H.A., Pelletier, D.A., Hurst, G.B. and Escalante-Semerena, J.C. (2012). System-wide studies of N-lysine acetylation in Rhodopseudomonas palustris reveal substrate specificity of protein acetyltransferases. J. Biol. Chem. 287, 15590-15601.

Cui, T.Z., Conte, A., Fox, J.L., Zara, V. and Winge D,R. (2014). Modulation of the respiratory supercomplexes in yeast: enhanced formation of cytochrome oxidase increases the stability and abundance of respiratory supercomplexes. J. Biol. Chem. 289, 6133-6141.

Cui, W., Rohrs, H.W., Gross, M.L. (2011). Top-down mass spectrometry: recent developments, applications and perspectives. Analyst 136, 3854-3864.

Curreem, S.O., Watt, R.M., Lau, S.K. and Woo, P.C. (2012). Two-dimensional gel electrophoresis in bacterial proteomics. Protein Cell 3, 346-363.

Cvetkovic, A., Menon, A.L., Thorgersen, M.P., Scott, J.W., Poole, F.L. 2nd, Jenney, F.E. Jr., Lancaster, W.A., Praissman, J.L., Shanmukh, S., Vaccaro, B.J., Trauger, S.A., Kalisiak, E., Apon, J.V., Siuzdak, G., Yannone, S.M., Tainer, J.A. and Adams, M.W. (2010). Microbial metalloproteomes are largely uncharacterized. Nature 466, 779-782.

Dagda, R.K., Sultana, T. and Lyons-Weiler, J. (2010). Evaluation of the Consensus of Four Peptide Identification Algorithms for Tandem Mass Spectrometry Based Proteomics. J. Proteomics Bioinform. 3, 39-47.

Dalbey, R.E., Kuhn, A., Zhu, L. and Kiefer, D. (2014). The membrane insertase YidC. Biochim. Biophys. Acta 1843, 1489-1496.

Dancik, V., Addona, T. A., Clauser, K.R., Vath, J.E. and Pevzner, P.A. (1999). De novo peptide sequencing via tandem mass spectrometry. J. Comput. Biol. 6, 327-342.

Dash, B.P., Alles, M., Bundschuh, F.A., Richter, O.M. and Ludwig, B. (2015). Protein chaperones mediating copper insertion into the CuA site of the $a_{3}$-type cytochrome $c$ oxidase of Paracoccus denitrificans. Biochim. Biophys. Acta 1847, 202-211.

Davies, K.M., Anselmi, C., Wittig, I., Faraldo-Gómez, J.D. and Kühlbrandt, W. (2012). Structure of the yeast F1Fo-ATP synthase dimer and its role in shaping the mitochondrial cristae. Proc. Natl. Acad. Sci. U S A 109, 13602-13607.

de Hoffmann, E. and Stroobant, V. (2007). Mass Spectrometry: Principles and Applications. Wiley.

De Rasmo D., Palmisano, G., Scacco, S., Technikova-Dobrova, Z., Panelli, D., Cocco, T., Sardanelli, A.M., Gnoni, A., Micelli, L., Trani, A., Di Luccia, A. and Papa, S.(2010). Phosphorylation pattern of the NDUFS4 subunit of complex I of the mammalian respiratory chain. Mitochondrion 10, 464471.

De Rasmo, D., Signorile, A., Santeramo, A., Larizza, M., Lattanzio, P., Capitanio, G. and Papa, S. (2015) Intramitochondrial adenylyl cyclase controls the turnover of nuclear-encoded subunits and activity of mammalian complex I of the respiratory chain. Biochim. Biophys. Acta 1853, 183-191. de Souza, G.A. and Wiker, H.G. (2011). A proteomic view of mycobacteria. Proteomics 11, 3118-3127. 
Dengjel, J., Kratchmarova, I. and Blagoev, B. (2010). Mapping protein-protein interactions by quantitative proteomics. Methods Mol. Biol. 658, 267-278.

Desmurs, M., Foti, M., Raemy, E., Vaz, F.M., Martinou, J.C., Bairoch, A. and Lane L. (2015). C11orf83, a mitochondrial cardiolipin-binding protein involved in $b c_{1}$ complex assembly and supercomplex stabilization. Mol. Cell Biol. 35, 1139-1156.

Douglas, D.J., Frank, A.J. and Mao, D. (2005). Linear ion traps in mass spectrometry. Mass. Spectrom. Rev. 24, 1-29.

Dresler, J., Klimentova, J. and Stulik, J. (2011) Francisella tularensis membrane complexome by blue native/SDS-PAGE. J. Proteomics 75, 257-269.

Dröse, S., Krack, S., Sokolova, L., Zwicker, K., Barth, H.D., Morgner, N., Heide, H., Steger, M., Nübel, E., Zickermann, V., Kerscher, S., Brutschy, B., Radermacher, M. and Brandt, U. (2011). Functional dissection of the proton pumping modules of mitochondrial complex I. PLoS Biol. 9:e1001128.

Dröse, S., Brandt, U. and Wittig, I. (2014). Mitochondrial respiratory chain complexes as sources and targets of thiol-based redox-regulation. Biochim. Biophys. Acta 1844, 1344-1354.

du Plessis, D.J., Nouwen, N. and Driessen, A.J. (2011). The Sec translocase. Biochim. Biophys. Acta 1808, 851-865.

Duarte, M. and Videira, A. (2009). Effects of mitochondrial complex III disruption in the respiratory chain of Neurospora crassa. Mol. Microbiol. 72, 246-258.

Dudkina, N.V., Eubel, H., Keegstra, W., Boekema, E.J. and Braun, H.P. (2005a). Structure of a mitochondrial supercomplex formed by respiratory-chain complexes I and III. Proc. Natl. Acad. Sci. U S A 102, 3225-3229.

Dudkina, N.V., Heinemeyer, J., Keegstra, W., Boekema, E.J. and Braun, H.P. (2005b). Structure of dimeric ATP synthase from mitochondria: an angular association of monomers induces the strong curvature of the inner membrane. FEBS Lett. 579, 5769-5772.

Dudkina, N.V., Kouril, R., Peters, K., Braun, H.P. and Boekema, E.J. (2010). Structure and function of mitochondrial supercomplexes. Biochim. Biophys. Acta 1797, 664-670.

Eichacker, L.A., Granvogl, B., Mirus, O., Müller, B.C., Miess, C. and Schleiff, E. (204). Hiding behind hydrophobicity. Transmembrane segments in mass spectrometry. J. Biol. Chem. 279, 50915-50922.

Eichacker, L.A., Weber, G., Sukop-Köppel, U. and Wildgruber, R. (2015). Free flow electrophoresis for separation of native membrane protein complexes. Methods Mol. Biol. 1295, 415-425.

Ekici, S., Pawlik, G., Lohmeyer, E., Koch, H.G. and Daldal, F. (2012). Biogenesis of $c b b_{3}$-type cytochrome $c$ oxidase in Rhodobacter capsulatus. Biochim. Biophys. Acta. 1817, 898-8910.

Elias, D.A., Monroe, M.E., Marshall, M.J., Romine, M.F., Belieav, A.S., Fredrickson, J.K., Anderson, G.A., Smith, R.D. and Lipton, M.S. (2005). Global detection and characterization of hypothetical proteins in Shewanella oneidensis MR-1 using LC-MS based proteomics. Proteomics 5, 31203130.

Eng, J.K., McCormack, A.L. and Yates, J.R. (1994). An approach to correlate tandem mass spectral data of peptides with amino acid sequences in a protein database. J. Am. Soc. Mass Spectrom. 5, 976989.

Erhardt, H., Steimle, S., Muders, V., Pohl, T., Walter, J. and Friedrich, T. (2012). Disruption of individual nuo-genes leads to the formation of partially assembled NADH:ubiquinone oxidoreductase (complex I) in Escherichia coli. Biochim. Biophys. Acta 1817, 863-871.

Erhardt, H., Dempwolff, F., Pfreundschuh, M., Riehle, M., Schäfer, C., Pohl, T., Graumann, P. and Friedrich, T. (2014). Organization of the Escherichia coli aerobic enzyme complexes of oxidative phosphorylation in dynamic domains within the cytoplasmic membrane. Microbiologyopen 3, 316326.

Ermler, U., Grabarse, W., Shima, S., Goubeaud, M. and Thauer, R.K. (1997). Crystal structure of methyl-coenzyme M reductase: the key enzyme of biological methane formation. Science 278, 1457-1462.

Eubel, H., Heinemeyer, J., Sunderhaus, S. and Braun, H.P. (2004). Respiratory chain supercomplexes in plant mitochondria. Plant Physiol. Biochem. 42, 937-942.

Everberg, H., Leiding, T., Schiöth, A., Tjerneld, F. and Gustavsson, N. (2006). Efficient and nondenaturing membrane solubilization combined with enrichment of membrane protein complexes by detergent/polymer aqueous two-phase partitioning for proteome analysis. J. Chromatogr. A 1122, 35-46. 
Everberg, H., Gustavasson, N. and Tjerned, F. (2008). Enrichment of membrane proteins by partitioning in detergent/polymer aqueous two-phase systems. Methods Mol. Biol. 424, 403-412.

Eymann, C., Dreisbach, A., Albrecht, D., Bernhardt, J., Becher, D., Gentner, S., Tam le, T., Büttner, K., Buurman, G., Scharf, C., Venz, S., Völker, U. and Hecker, M. (2004). A comprehensive proteome map of growing Bacillus subtilis cells. Proteomics 4, 2849-2876.

Eymann, C., Becher, D., Bernhardt, J., Gronau, K., Klutzny, A. and Hecker, M. (2007). Dynamics of protein phosphorylation on Ser/Thr/Tyr in Bacillus subtilis. Proteomics 7, 3509-3526.

Fahey, R.C. (2013). Glutathione analogs in prokaryotes. Biochim. Biophys. Acta 1830, 3182-3198.

Fang, R., Elias, D.A., Monroe, M.E., Shen, Y., McIntosh, M., Wang, P., Goddard, C.D., Callister, S.J., Moore, R.J., Gorby, Y.A., Adkins, J.N., Fredrickson, J.K., Lipton, M.S. and Smith, R.D. (2006). Differential label-free quantitative proteomic analysis of Shewanella oneidensis cultured under aerobic and suboxic conditions by accurate mass and time tag approach. Mol. Cell. Proteomics. 5, 714-725.

Farhoud, M.H., Wessels, H.J., Steenbakkers, P.J., Mattijssen, S., Wevers, R.A., van Engelen, B.G., Jetten, M.S., Smeitink, J.A., van den Heuvel, L.P. and Keltjens, J.T. (2005). Protein complexes in the archaeon Methanothermobacter thermautotrophicus analyzed by blue native/SDS-PAGE and mass spectrometry. Mol. Cell. Proteomics 4, 1653-1663.

Farhoud, M.H. (2011). Disease biology of mitochondrial complex-I: proteomic insights. PhD thesis. Radboud University Nijmegen.

Faust, K., Lahti, L., Gonze, D., de Vos, W.M. and Raes, J. (2015). Metagenomics meets time series analysis: unraveling microbial community dynamics. Curr. Opin. Microbiol. 25, 56-66.

Fearnley, I.M., Carroll, J. and Walker, J.E. (2007). Proteomic analysis of the subunit composition of complex I (NADH:ubiquinone oxidoreductase) from bovine heart mitochondria. Methods Mol. Biol. 357, 103-125.

Feng, S., Powell, S.M., Wilson, R. and Bowman, J.P. (2015). Proteomic Insight into Functional Changes of Proteorhodopsin-Containing Bacterial Species Psychroflexus torquis under Different Illumination and Salinity Levels. J. Proteome Res. 14, 3848-3858.

Feng, Y., De Franceschi, G., Kahraman, A., Soste, M., Melnik, A., Boersema, P.J., de Laureto, P.P., Nikolaev, Y., Oliveira, A.P. and Picotti, P. (2014). Global analysis of protein structural changes in complex proteomes. Nat. Biotechnol. 32, 1036-1044.

Ferrer, M., Golyshina, O.V., Beloqui, A., Golyshin, P.N. and Timmis, K.N. (2007). The cellular machinery of Ferroplasma acidiphilum is iron-protein-dominated. Nature 445, 91-94.

Fischer, F. and Poetsch, A. (2006). Protein cleavage strategies for an improved analysis of the membrane proteome. Proteome Sci. 4:2.

Fischer, F., Wolters, D., Rögner, M. and Poetsch, A. (2005). Toward the complete membrane proteome: high coverage of integral membrane proteins through transmembrane peptide detection. Mol. Cell. Proteomics 5, 444-453.

Fishov, I. and Norris, V. (2012). Membrane heterogeneity created by transertion is a global regulator in bacteria. Curr. Opin. Microbiol. 15, 724-730.

Fontana, A., de Laureto, P.P., Spolaore, B., Frare, E., Picotti, P. and Zambonin, M. (2004). Probing protein structure by limited proteolysis. Acta Biochim. Pol. 51, 299-321.

Fontana, A., de Laureto, P.P., Spolaore, B. and Frare E. (2012). Identifying disordered regions in proteins by limited proteolysis. Methods Mol. Biol. 896, 297-318.

Formosa, L.E., Mimaki, M., Frazier, A.E., McKenzie, M., Stait, T.L., Thorburn, D.R., Stroud, D.A. and Ryan, M.T. (2015). Characterization of mitochondrial FOXRED1 in the assembly of respiratory chain complex I. Hum. Mol. Genet. 24, 2952-2965.

Frank, A. and Pevzner, P. (2005). PepNovo: de novo peptide sequencing via probabilistic network modeling. Anal. Chem. 77, 964-973.

Fränzel, B. and Wolters, D.A. (2011). Advanced MudPIT as a next step toward high proteome coverage. Proteomics 11, 3651-3656.

Fränzel, B., Poetsch, A., Trötschel, C., Persicke, M., Kalinowski, J. and Wolters, D.A. (2010). Quantitative proteomic overview on the Corynebacterium glutamicum l-lysine producing strain DM1730. J. Proteomics 73, 2336-2353. 
Freeman, E. and Ivanov, A.R. (2011). Proteomics under pressure: development of essential sample preparation techniques in proteomics using ultrahigh hydrostatic pressure. J. Proteome Res. 10, 5536-5546.

Frezza, C., Cipolat, S., Martins de Brito, O., Micaroni, M., Beznoussenko, G.V., Rudka, T., Bartoli, D., Polishuck, R.S., Danial, N.N., De Strooper, B., Scorrano, L. (2006). OPA1 controls apoptotic cristae remodeling independently from mitochondrial fusion. Cell 126, 177-189.

Frielingsdorf, S., Schubert, T., Pohlmann, A., Lenz, O. and Friedrich, B. (2011). A trimeric supercomplex of the oxygen-tolerant membrane-bound [NiFe]-hydrogenase from Ralstonia eutropha H16. Biochemistry 50, 10836-10843.

Fröbel, J., Rose, P. and Müller, M. (2012). Twin-arginine-dependent translocation of folded proteins. Philos. Trans. R. Soc. Lond. B Biol. Sci. 367, 1029-1046.

Fu, H., Yuan, J. and Gao, H. (2015). Microbial oxidative stress response: Novel insights from environmental facultative anaerobic bacteria. Arch. Biochem. Biophys. 584, 28-35.

Fujiki, Y., Hubbard, A.L., Fowler, S. and Lazarow, P.B. (1982). Isolation of intracellular membranes by means of sodium carbonate treatment: application to endoplasmic reticulum. J. Cell. Biol. 93, 97-102.

Fujita, Y. (2009). Carbon catabolite control of the metabolic network in Bacillus subtilis. Biosci. Biotechnol. Biochem. 73, 245-259.

Fukuyama, Y., Iwamoto, S. and Tanaka, K. (2006). Rapid sequencing and disulfide mapping of peptides containing disulfide bonds by using 1,5-diaminonaphthalene as a reductive matrix. J. Mass Spectrom. 41, 191-201.

Galkin, A., Meyer, B., Wittig, I., Karas, M., Schägger, H., Vinogradov, A. and Brandt, U. (2008). Identification of the mitochondrial ND3 subunit as a structural component involved in the active/deactive enzyme transition of respiratory complex I. J. Biol. Chem. 283, 20907-20913.

Gallien, S., Duriez, E. and Domon, B. (2011). Selected reaction monitoring applied to proteomics. J. Mass Spectrom. 46, 298-312.

Gama, M.R., Collins, C.H. and Bottoli, C.B. (2013). Nano-liquid chromatography in pharmaceutical and biomedical research. J. Chromatogr. Sci. 51, 694-703.

García Montes de Oca, L.Y., Chagolla-López, A., González de la Vara, L., Cabellos-Avelar, T., GómezLojero, C. and Gutiérrez Cirlos, E.B. (2012). The composition of the Bacillus subtilis aerobic respiratory chain supercomplexes. J. Bioenerg. Biomembr. 44, 473-486.

Ge, R., Sun, X., Xiao, C., Yin, X., Shan, W., Chen, Z. and He Q.Y. (2011). Phosphoproteome analysis of the pathogenic bacterium Helicobacter pylori reveals over-representation of tyrosine phosphorylation and multiply phosphorylated proteins. Proteomics 11, 1449-1461.

Geer, L.Y., Markey, S.P., Kowalak, J.A., Wagner, L., Xu, M., Maynard, D.M., Yang, X., Shi, W. and Bryant, S.H. (2004). Open mass spectrometry search algorithm. J. Proteome Res. 3, 958-964.

Genova, M.L. and Lenaz, G. (2013). A critical appraisal of the role of respiratory supercomplexes in mitochondria. Biol. Chem. 394, 631-639.

Genova, M.L. and Lenaz, G. (2014). Functional role of mitochondrial respiratory supercomplexes. Biochim. Biophys. Acta 1837, 427-443.

Gerber, G.K. (2014). The dynamic microbiome. FEBS Lett. 588, 4131-4139.

Gerber, S.A., Rush, J., Stemman, O., Kirschner, M.W. and Gygi, S.P. (2003). Absolute quantification of proteins and phosphoproteins from cell lysates by tandem MS. Proc. Natl. Acad. Sci. U S A 100, 6940-6945.

Giese, H., Ackermann, J., Heide, H., Bleier, L., Dröse, S., Wittig, I., Brandt, U. and Koch, I. (2015). NOVA: a software to analyze complexome profiling data. Bioinformatics 31, 440-441.

Gil Borlado, M.C., Moreno Lastres, D., Gonzalez Hoyuela, M., Moran, M., Blazquez, A., Pello, R., Marin Buera, L., Gabaldon, T., Garcia Peñas, J.J., Martín, M.A., Arenas, J. and Ugalde, C. (2010). Impact of the mitochondrial genetic background in complex III deficiency. PLoS One 5 pii: e12801.

Gillette, M. . and Carr, S.A. (2013). Quantitative analysis of peptides and proteins in biomedicine by targeted mass spectrometry. Nat. Methods 10, 28-34.

Gilmore, J.M. and Washburn, M.P. (2010). Advances in shotgun proteomics and the analysis of membrane proteomes. J. Proteomics 73, 2078-2091.

Gingras, A.C., Gstaiger, M., Raught, B. and Aebersold, R. (2007). Analysis of protein complexes using mass spectrometry. Nat. Rev. Mol. Cell Biol. 8, 645-654. 
Glew, M.D., Veith, P.D., Chen, D., Seers, C.A., Chen, Y.Y.and Reynolds, E.C. (2014). Blue nativePAGE analysis of membrane protein complexes in Porphyromonas gingivalis. J. Proteomics 110, 72-92.

Godzik, A. (2011). Metagenomics and the protein universe. Curr. Opin. Struct. Biol. 21, 398-403.

Gómez, L.A., Monette, J.S., Chavez, J.D., Maier, C.S. and Hagen, T.M. (2009). Supercomplexes of the mitochondrial electron transport chain decline in the aging rat heart. Arch. Biochem. Biophys. 490, 30-35.

Goosens, V.J., Otto, A., Glasner, C., Monteferrante, C.C., van der Ploeg, R., Hecker, M., Becher, D. and van Dijl, J.M. (2013). Novel twin-arginine translocation pathway-dependent phenotypes of Bacillus subtilis unveiled by quantitative proteomics. J. Proteome Res. 12, 796-807.

Goosens, V.J., Monteferrante, C.G. and van Dijl, J.M. (2014a). Co-factor insertion and disulfide bond requirements for twin-arginine translocase-dependent export of the Bacillus subtilis Rieske protein QcrA. J. Biol. Chem. 289, 13124-13131.

Goosens, V.J., Monteferrante, C.G. and van Dijl, J.M. (2014b).The Tat system of Gram-positive bacteria. Biochim. Biophys. Acta 1843, 1698-1706.

Görg, A., Weiss, W. and Dunn, M.J. (2004). Current two-dimensional electrophoresis technology for proteomics. Proteomics 4, 3665-3685.

Gorman, J.J., Ferguson, B.L. and Nguyen, T.B. (1996). Use of 2,6-dihydroxyacetophenone for analysis of fragile peptides, disulphide bonding and small proteins by matrix-assisted laser desorption/ionization. Rapid Commun. Mass Spectrom. 10, 529-536.

Götzke, H., Muheim, C., Altelaar, A.F., Heck, A.J., Maddalo, G. and Daley, D.O. (2015). Identification of putative substrates for the periplasmic chaperone YfgM in Escherichia coli using quantitative proteomics. Mol. Cell. Proteomics 14, 216-226.

Gouw, J.W. and Krijgsveld, J. (2012). MSQuant: a platform for stable isotope-based quantitative proteomics. Methods Mol. Biol. 893, 511-522.

Grabarse, W., Mahlert, F., Duin, E.C., Goubeaud, M., Shima, S., Thauer, R.K., Lamzin, V. and Ermler, U. (2001). On the mechanism of biological methane formation: structural evidence for conformational changes in methyl-coenzyme M reductase upon substrate binding. J. Mol. Biol. 309, 315-330.

Grangeasse, C., Cozzone, A.J., Deutscher, J. and Mijakovic, I. (2007). Tyrosine phosphorylation: an emerging regulatory device of bacterial physiology. Trends Biochem. Sci. 32, 86-94.

Grangeasse, C., Stülke, J. and Mijakovic, I. (2015). Regulatory potential of post-translational modifications in bacteria. Front. Microbiol. 6:500.

Grein, F., Ramos, A.R., Venceslau, S.S., Pereira, I.A. (2013). Unifying concepts in anaerobic respiration: insights from dissimilatory sulfur metabolism. Biochim. Biophys. Acta 1827, 145-160.

Grimaldi, S., Schoepp-Cothenet, B., Ceccaldi, P., Guigliarelli, B. and Magalon A. (2013). The prokaryotic Mo/W-bisPGD enzymes family: a catalytic workhorse in bioenergetic. Biochim. Biophys. Acta 1827, 1048-1085.

Grossmann, J., Roos, F. F., Cieliebak, M., Liptak, Z., Mathis, L.K., Muller, M., Gruissem, W. and Baginsky, S. (2005). AUDENS: a tool for automated peptide de novo sequencing. J. Proteome. Res. 4, 1768-1774.

Gu, L., Chen, Y., Wang, Q.T., Li, X., Mi, K. and Deng, H. (2015). Functional Characterization of Sirtuin-like Protein in Mycobacterium smegmatis. J. Proteome Res. 14, 4441-4449.

Guan, K.L. and Xiong, Y. (2011). Regulation of intermediary metabolism by protein acetylation. Trends Biochem. Sci. 36, 108-116.

Guan, K.L., Yu, W., Lin, Y., Xiong, Y. and Zhao, S. (2010). Generation of acetyllysine antibodies and affinity enrichment of acetylated peptides. Nat. Protoc. 5, 1583-1595.

Gurumoorthy, P. and Ludwig, B. (2015). Deciphering protein-protein interactions during the biogenesis of cytochrome $c$ oxidase from Paracoccus denitrificans. FEBS J. 282, 537-549.

Guthals, A. and Bandeira, N. (2012). Peptide identification by tandem mass spectrometry with alternate fragmentation modes. Mol. Cell. Proteomics 11, 550-557.

Gygi, S.P., Rist, B., Gerber, S.A., Turecek, F., Gelb, M.H. and Aebersold, R. (199) Quantitative analysis of complex protein mixtures using isotope-coded affinity tags. Nat. Biotechnol. 17, 994-999. 
Habersetzer, J., Larrieu, I., Priault, M., Salin, B., Rossignol, R., Brèthes, D. and Paumard, P. (2013). Human $\mathrm{F}_{1} \mathrm{~F}_{0}$ ATP synthase, mitochondrial ultrastructure and OXPHOS impairment: a (super-) complex matter? PLoS One 8:e75429.

Hakhverdyan, Z., Domanski, M., Hough, L.E., Oroskar, A.A., Oroskar, A.R., Keegan, S., Dilworth, D.J., Molloy, K.R., Sherman, V., Aitchison, J.D., Fenyö, D., Chait, B.T., Jensen, T.H., Rout, M.P. and LaCava, J. (2015). Rapid, optimized interactomic screening. Nat. Methods 12, 553-560.

Han, H., Hemp, J., Pace, L.A., Ouyang, H., Ganesan, K., Roh, J.H., Daldal, F., Blanke, S.R. and Gennis, R.B. (2011). Adaptation of aerobic respiration to low $\mathrm{O}_{2}$ environments. Proc. Natl. Acad. Sci. U S A 108, 14109-14114.

Han, M.J. and Lee SY. (2006). The Escherichia coli proteome: past, present, and future prospects. Microbiol. Mol. Biol. Rev. 70, 362-439.

Hannappel, A., Bundschuh, F.A. and Ludwig, B. (2012). Role of Surf1 in heme recruitment for bacterial COX biogenesis. Biochim. Biophys. Acta 1817, 928-937.

Hartmann, T., Schwanhold, N. and Leimkühler, S. (2015).Assembly and catalysis of molybdenum or tungsten-containing formate dehydrogenases from bacteria. Biochim. Biophys. Acta 1854, 10901100.

Hasan, S.S., Yamashita, E. and Cramer, W.A. (2013). Transmembrane signaling and assembly of the cytochrome $b_{6} f$-lipidic charge transfer complex. Biochim. Biophys. Acta 1827, 1295-1308.

Hasan, S.S., Proctor, E.A., Yamashita, E., Dokholyan, N.V. and Cramer, W.A. (2014).Traffic within the cytochrome $b_{6} f$ lipoprotein complex: gating of the quinone portal. Biophys. J. 107, 1620-1628.

Haselberg, R., de Jong, G.J. and Somsen, G.W. (2013). CE-MS for the analysis of intact proteins 20102012. Electrophoresis 34, 99-112.

Hatzixanthis, K., Richardson, D.J. and Sargent, F. (2005). Chaperones involved in assembly and export of N-oxide reductases. Biochem. Soc. Trans. 33, 124-126.

Heide, H., Bleier, L., Steger, M., Ackermann, J., Dröse, S., Schwamb, B., Zörnig, M., Reichert, A.S., Koch, I., Wittig, I. and Brandt, U. (2012). Complexome profiling identifies TMEM126B as a component of the mitochondrial complex I assembly complex. Cell Metab. 16, 538-549.

Heide, H. and Wittig, I. (2013). Methods to analyse composition and dynamics of macromolecular complexes. Biochem. Soc. Trans. 41, 1235-1241.

Heimann, A.C., Blodau, C., Postma, D., Larsen, F., Viet, P.H., Nhan, P.Q., Jessen, S., Duc, M.T., Hue N.T. and Jakobsen, R. (2007). Hydrogen thresholds and steady-state concentrations associated with microbial arsenate respiration. Environ. Sci. Technol. 41, 2311-2317.

Heintz, D., Gallien, S., Wischgoll, S., Ullmann, A.K., Schaeffer, C., Kretzschmar, A.K., van Dorsselaer, A. and Boll, M. (2009). Differential membrane proteome analysis reveals novel proteins involved in the degradation of aromatic compounds in Geobacter metallireducens. Mol. Cell. Proteomics 8, 2159-2169.

Helbig, A.O., de Groot, M.J., van Gestel, R.A., Mohammed, S., de Hulster, E.A., Luttik, M.A., DaranLapujade, P., Pronk, J.T., Heck, A.J. and Slijper, M. (2009). A three-way proteomics strategy allows differential analysis of yeast mitochondrial membrane protein complexes under anaerobic and aerobic conditions. Proteomics 9, 4787-4798.

Helbig, A.O., Heck, A.J. and Slijper, M. (2010). Exploring the membrane proteome--challenges and analytical strategies. J. Proteomics 73, 868-878.

Helling, S., Schmitt, E., Joppich, C., Schulenborg, T., Müllner, S., Felske-Müller, S., Wiebringhaus, T., Becker, G., Linsenmann, G., Sitek, B., Lutter, P., Meyer, H.E. and Marcus, K. (2006). 2-D differential membrane proteome analysis of scarce protein samples. Proteomics 6, 4506-4513.

Helling, S., Vogt, S., Rhiel, A., Ramzan, R., Wen, L., Marcus, K. and Kadenbach, B. (2008). Phosphorylation and kinetics of mammalian cytochrome c oxidase. Mol. Cell. Proteomics 7, 17141724.

Helling, S., Hüttemann, M., Kadenbach, B., Ramzan, R., Vogt, S. and Marcus, K.(2012a). Discovering the phosphoproteome of the hydrophobic cytochrome $c$ oxidase membrane protein complex. Methods Mol. Biol. 893, 345-358.

Helling, S., Hüttemann, M., Ramzan, R., Kim, S.H., Lee, I., Müller, T., Langenfeld, E., Meyer, H.E., Kadenbach, B., Vogt, S. and Marcus, K. (2012b). Multiple phosphorylations of cytochrome $c$ oxidase and their functions. Proteomics 12, 950-959. 
Hemp, J., Gennis, R.B. (2008). Diversity of the heme-copper superfamily in archaea: insights from genomics and structural modeling. Results Probl. Cell. Differ. 45, 1-31.

Hendrickson, C.L., Quinn, J.P., Kaiser, N.K., Smith, D.F., Blakney, G.T., Chen, T., Marshall, A.G., Weisbrod, C.R. and Beu, S.C. (2015). 21 Tesla Fourier Transform Ion Cyclotron Resonance Mass Spectrometer: A National Resource for Ultrahigh Resolution Mass Analysis. J. Am. Soc. Mass Spectrom. 26, 1626-1632.

Hendrickx, S., Adams, E. and Cabooter, D. (2015). Recent advances in the application of hydrophilic interaction chromatography for the analysis of biological matrices. Bioanalysis 7, 2927-2945.

Hentchel, K.L. and Escalante-Semerena, J.C. (2015). Acylation of Biomolecules in Prokaryotes: a Widespread Strategy for the Control of Biological Function and Metabolic Stress. Microbiol. Mol. Biol. Rev. 79, 321-346.

Henzel, W.J., Billeci, T.M., Stults, J.T., Wong, S.C., Grimley, C. and Watanabe, C. (1993). Identifying proteins from two-dimensional gels by molecular mass searching of peptide fragments in protein sequence databases. Proc. Natl. Acad. Sci. U S A. 90, 5011-5015.

Henzel, W.J., Watanabe, C. and Stults, J.T. (2003). Protein identification: the origins of peptide mass fingerprinting. J. Am. Soc. Mass Spectrom. 14, 931-942.

Hillenkamp, F., Karas, M., Beavis, R.C. and Chait, B.T. (1991). Matrix-assisted laser desorption/ ionization mass spectrometry of biopolymers. Anal. Chem. 63, 1193A-1203A.

Hillenkamp, F. and Karas, M. (1990). Mass spectrometry of peptides and proteins by matrix-assisted ultraviolet laser desorption/ionization. Methods Enzymol. 193, 280-295.

Hillion, M. and Antelmann, H. (2015). Thiol-based redox switches in prokaryotes. Biol. Chem. 396, 415-444.

Hitchcock, A., Hall, S.J., Myers, J.D., Mulholland, F., Jones, M.A. and Kelly, D.J. (2010). Roles of the twin-arginine translocase and associated chaperones in the biogenesis of the electron transport chains of the human pathogen Campylobacter jejuni. Microbiology 156, 2994-3010.

Hochgräfe, F., Mostertz, J., Pöther, D.C., Becher, D., Helmann, J.D. and Hecker, M. (2007). Scysteinylation is a general mechanism for thiol protection of Bacillus subtilis proteins after oxidative stress. J. Biol. Chem. 282, 25981-25985.

Hoffmann, J., Sokolova, L., Preiss, L., Hicks, D.B., Krulwich, T.A., Morgner, N., Wittig, I., Schägger, H., Meier, T. and Brutschy, B. (2010). ATP synthases: cellular nanomotors characterized by LILBID mass spectrometry. Phys. Chem. Chem. Phys. 12, 13375-13382.

Hogan, J.M., Pitteri, S.J., Chrisman, P.A. and McLuckey, S.A. (2005). Complementary structural information from a tryptic N-linked glycopeptide via electron transfer ion/ion reactions and collision-induced dissociation. J. Proteome Res. 4, 628-632.

Hoke, D.E., Zhang, K., Egan, S., Hatfaludi, T., Buckle, A.M. and Adler, B. (2011). Membrane proteins of Pseudoalteromonas tunicata during the transition from planktonic to extracellular matrixadherent state. Environ. Microbiol. Rep. 3, 405-413.

Hopkins, A., Buchanan, G. and Palmer, T. (2014). Role of the twin arginine protein transport pathway in the assembly of the Streptomyces coelicolor cytochrome $b c_{1}$ complex. J. Bacteriol. 196, 50-59.

Hopper, J.T. and Robinson, C.V. (2014). Mass spectrometry quantifies protein interactions--from molecular chaperones to membrane porins. Angew. Chem. Int. Ed. Engl. 53, 14002-14015.

Hopper, J.T., Yu, Y.T., Li, D., Raymond, A., Bostock, M., Liko, I., Mikhailov, V., Laganowsky, A., Benesch, J.L., Caffrey, M., Nietlispach, D. and Robinson, C.V. (2013). Detergent-free mass spectrometry of membrane protein complexes. Nat. Methods. 10, 1206-1208.

Huang, T.Y. and McLuckey, S.A. (2010). Gas-phase chemistry of multiply charged bioions in analytical mass spectrometry. Annu. Rev. Anal. Chem. (Palo Alto Calif) 3, 365-385.

Hui, S., Silverman, J.M., Chen, S.S., Erickson, D.W., Basan, M., Wang, J., Hwa, T. and Williamson, J.R. (2015). Quantitative proteomic analysis reveals a simple strategy of global resource allocation in bacteria. Mol. Syst. Biol. 11:784.

Hutchings, M.I. , Palmer, T., Harrington, D.J. and Sutcliffe, I.C. (2009). Lipoprotein biogenesis in Gram-positive bacteria: knowing when to hold 'em, knowing when to fold 'em. Trends Microbiol. 17, 13-21.

Hüttemann, M., Helling, S., Sanderson, T.H., Sinkler, C., Samavati, L., Mahapatra, G., Varughese, A., Lu, G., Liu, J., Ramzan, R., Vogt, S., Grossman, L.I., Doan, J.W., Marcus, K. and Lee, I. (2012). Regulation of mitochondrial respiration and apoptosis through cell signaling: cytochrome $c$ oxidase 
and cytochrome $c$ in ischemia/reperfusion injury and inflammation. Biochim. Biophys. Acta 1817, 598-609.

Huynen, M.A., Mühlmeister, M., Gotthardt, K., Guerrero-Castillo, S. and Brandt, U. (2015). Evolution and structural organization of the mitochondrial contact site (MICOS) complex and the mitochondrial intermembrane space bridging (MIB) complex. Biochim. Biophys. Acta 1863, 91101.

Hyung, S.J. and Ruotolo, B.T. (2012). Integrating mass spectrometry of intact protein complexes into structural proteomics. Proteomics 12, 1547-1564.

Iacob, R.E. and Engen, J.R. (2012). Hydrogen exchange mass spectrometry: are we out of the quicksand? J. Am. Soc. Mass. Spectrom. 23, 1003-1010.

Imlay, J.A. (2013). The molecular mechanisms and physiological consequences of oxidative stress: lessons from a model bacterium. Nat. Rev. Microbiol. 11, 443-454.

Ishihama, Y., Oda, Y., Tabata, T., Sato, T., Nagasu, T., Rappsilber, J. and Mann, M. (2005). Exponentially modified protein abundance index (emPAI) for estimation of absolute protein amount in proteomics by the number of sequenced peptides per protein. Mol. Cell. Proteomics 4, $1265-1272$.

Issaq, H. and Veenstra, T. (2008). Two-dimensional polyacrylamide gel electrophoresis (2D-PAGE): advances and perspectives. Biotechniques 44, 697-700.

James, P., Quadroni, M., Carafoli, E. and Gonnet, G. (1993). Protein identification by mass profile fingerprinting. Biochem. Biophys. Res. Commun. 195, 58-64.

Jeong, K., Kim, S. and Pevzner, P.A. (2013). UniNovo: a universal tool for de novo peptide sequencing. Bioinformatics 29, 1953-1962.

Jers, C., Kobir, A., Søndergaard, E.O., Jensen, P.R. and Mijakovic, I. (2011). Bacillus subtilis twocomponent system sensory kinase DegS is regulated by serine phosphorylation in its input domain. PLoS One 6:e14653.

Jiang, C.Y., Liu, L.J., Guo, X., You, X.Y., Liu, S.J. and Poetsch, A. (2014). Resolution of carbon metabolism and sulfur-oxidation pathways of Metallosphaera cuprina Ar-4 via comparative proteomics. J. Proteomics 109, 276-289.

Jo, I., Chung, I.Y., Bae, H.W., Kim, J.S., Song, S., Cho, Y.H. and Ha N.C. (2015). Structural details of the OxyR peroxide-sensing mechanism. Proc. Natl. Acad. Sci. U S A 112, 6443-6448.

Johnson, A.S., van Horck, S. and Lewis P.J. (2004). Dynamic localization of membrane proteins in Bacillus subtilis. Microbiology 150, 2815-2824.

Johnson, T.L., Sikora, A.E., Zielke, R.A. and Sandkvist, M. (2013). Fluorescence microscopy and proteomics to investigate subcellular localization, assembly, and function of the type II secretion system. Methods Mol. Biol. 966, 157-172.

Jones, J.D. and O'Connor, C.D. (2011). Protein acetylation in prokaryotes. Proteomics 11, 3012-3022.

Juhasz, P., Costello, C.E. and Biemann, K. (1993). Matrix-assisted laser desorption ionization mass spectrometry with 2-(4-hydroxyphenylazo)benzoic acid matrix. J. Am. Soc. Mass Spectrom. 4, 399409.

Kane, L.A. and Van Eyk, J.E. (2009). Post-translational modifications of ATP synthase in the heart: biology and function. J. Bioenerg. Biomembr. 41, 145-150.

Kane, L.A., Youngman, M.J., Jensen, R.E. and Van Eyk, J.E. (2010). Phosphorylation of the $F_{1} F_{0}$ ATP synthase beta subunit: functional and structural consequences assessed in a model system. Circ. Res. 106, 504-513.

Karabacak, N.M., Li, L., Tiwari, A., Hayward, L.J., Hong, P., Easterling, M.L. and Agar, J.N. (2009). Sensitive and specific identification of wild type and variant proteins from 8 to $669 \mathrm{kDa}$ using topdown mass spectrometry. Mol. Cell. Proteomics 8, 846-856.

Karas, M., Bachmann, D. and Hillenkamp, F. (1985). Influence of the wavelength in high-irradiance ultraviolet laser desorption mass spectrometry of organic molecules. Anal. Chem. 57, 2935-2939.

Kartal, B., de Almeida, N.M., Maalcke, W.J., Op den Camp, H.J., Jetten, M.S. and Keltjens, J.T. (2013). How to make a living from anaerobic ammonium oxidation. FEMS Microbiol. Rev. 37, 428-461.

Kashino, Y., Harayama, T., Pakrasi, H.B. and Satoh K. (2007). Preparation of membrane proteins for analysis by two-dimensional gel electrophoresis. J. Chromatogr. B Analyt. Technol. Biomed. Life Sci. 849, 282-292. 
Kaster, A.K., Moll, J., Parey, K. and Thauer, R.K. (2011). Coupling of ferredoxin and heterodisulfide reduction via electron bifurcation in hydrogenotrophic methanogenic archaea. Proc. Natl. Acad. Sci. U S A 108, 2981-2986.

Kero, F.A., Pedder, R.E. and Yost, R.A. (2004). Encyclopedia of Genetics, Genomics, Proteomics and Bioinformatics. John Wiley and Sons, Ltd.

Kettenbach, A.N., Rush, J. and Gerber S.A. (2011). Absolute quantification of protein and posttranslational modification abundance with stable isotope-labeled synthetic peptides. Nat. Protoc. 6 , 175-186.

Kim, D., Yu, B.J., Kim, J.A., Lee, Y.J., Choi, S.G., Kang, S. and Pan J.G. (2013). The acetylproteome of Gram-positive model bacterium Bacillus subtilis. Proteomics 13, 1726-1736.

Kim, G.W. and Yang, X.J. (2011). Comprehensive lysine acetylomes emerging from bacteria to humans. Trends Biochem. Sci. 36, 211-220.

Kim, J.H., Bothe, J.R., Alderson, T.R. and Markley, J.L. (2015). Tangled web of interactions among proteins involved in iron-sulfur cluster assembly as unraveled by NMR, SAXS, chemical crosslinking, and functional studies. Biochim. Biophys. Acta 1853, 1416-1428.

Kim, M.S., Jang, J., Ab Rahman, N.B., Pethe, K., Berry, E.A. and Huang L.S. (2015). Isolation and Characterization of a Hybrid Respiratory Supercomplex Consisting of Mycobacterium tuberculosis Cytochrome bcc and Mycobacterium smegmatis Cytochrome $a_{3}$. J. Biol. Chem. 290, 1435014360.

Kim, S.H., Kim, S.K., Jung, K.H., Kim, Y.K., Hwang, H.C., Ryu, S.G. and Chai, Y.G. (2013). Proteomic analysis of the oxidative stress response induced by low-dose hydrogen peroxide in Bacillus anthracis. J. Microbiol. Biotechnol. 23, 750-758.

Kislinger, T., Gramolini, A.O., MacLennan, D.H. and Emili, A. (2005). Multidimensional protein identification technology (MudPIT): technical overview of a profiling method optimized for the comprehensive proteomic investigation of normal and diseased heart tissue. J. Am. Soc. Mass Spectrom. 16, 1207-1220.

Kliman, M., May, J.C., McLean, J.A. (2011). Lipid analysis and lipidomics by structurally selective ion mobility-mass spectrometry. Biochim. Biophys. Acta 1811, 935-945.

Klodmann, J. and Braun, H.P. (2011). Proteomic approach to characterize mitochondrial complex I from plants. Phytochemistry 72, 1071-1080.

Klodmann, J., Sunderhaus, S., Nimtz, M., Jänsch, L. and Braun, H.P. (2010). Internal architecture of mitochondrial complex I from Arabidopsis thaliana. Plant Cell 22, 797-810.

Klodmann, J., Lewejohann, D. and Braun, H.P. (2011). Low-SDS Blue native PAGE. Proteomics 11, 1834-1839.

Kobayashi, K., Fujikawa, M. and Kozawa, T. (2014). Oxidative stress sensing by the iron-sulfur cluster in the transcription factor, SoxR. J. Inorg. Biochem. 133, 87-91.

Kohlmann, Y., Pohlmann, A., Otto, A., Becher, D., Cramm, R., Lütte, S., Schwartz, E., Hecker, M. and Friedrich, B. (2011). Analyses of soluble and membrane proteomes of Ralstonia eutropha H16 reveal major changes in the protein complement in adaptation to lithoautotrophy. J. Proteome Res. 10, 2767-2776.

Kohlmann, Y., Pohlmann, A., Schwartz, E., Zühlke, D., Otto, A., Albrecht, D., Grimmler, C., Ehrenreich, A., Voigt, B., Becher, D., Hecker, M., Friedrich, B. and Cramm, R. (2014). Coping with anoxia: a comprehensive proteomic and transcriptomic survey of denitrification. J. Proteome Res. 13, 4325-4338.

Konermann, L., Tong, X. and Pan, Y. (2008). Protein structure and dynamics studied by mass spectrometry: H/D exchange, hydroxyl radical labeling, and related approaches. J. Mass Spectrom. 43, 1021-1036.

Konermann, L., Pan, J. and Liu, Y.H. (2011). Hydrogen exchange mass spectrometry for studying protein structure and dynamics. Chem. Soc. Rev. 40, 1224-1234.

Konermann, L. and Pan, Y. (2012). Exploring membrane protein structural features by oxidative labeling and mass spectrometry. Expert Rev. Proteomics 9, 497-504.

Konijnenberg, A., Bannwarth, L., Yilmaz, D., Koçer, A., Venien-Bryan, C. and Sobott, F. (2015). Topdown mass spectrometry of intact membrane protein complexes reveals oligomeric state and sequence information in a single experiment. Protein Sci. 24, 1292-1300. 
Kosono, S., Tamura, M., Suzuki, S., Kawamura, Y., Yoshida, A., Nishiyama, M. and Yoshida M. (2015). Changes in the Acetylome and Succinylome of Bacillus subtilis in Response to Carbon Source. PLoS One 10:e0131169.

Kraft, B., Strous, M. and Tegetmeyer, H.E. (2011). Microbial nitrate respiration--genes, enzymes and environmental distribution. J. Biotechnol. 155, 104-117.

Kranz, R.G., Richard-Fogal, C., Taylor, J.S. and Frawley, E.R. (2009). Cytochrome $c$ biogenesis: mechanisms for covalent modifications and trafficking of heme and for heme-iron redox control. Microbiol. Mol. Biol. Rev. 73, 510-528.

Krause, F., Reifschneider, N.H., Vocke, D., Seelert, H., Rexroth, S. and Dencher, N.A. (2004). "Respirasome"-like supercomplexes in green leaf mitochondria of spinach. J. Biol. Chem. 279, 48369-48375.

Krause, F. (2006). Detection and analysis of protein-protein interactions in organellar and prokaryotic proteomes by native gel electrophoresis: (Membrane) protein complexes and supercomplexes. Electrophoresis 27, 2759-2781.

Krijgsveld, J., Ketting, R.F., Mahmoudi, T., Johansen, J., Artal-Sanz, M., Verrijzer, C.P., Plasterk, R.H. and Heck, A.J. (2003). Metabolic labeling of C. elegans and D. melanogaster for quantitative proteomics. Nat. Biotechnol. 21, 927-931.

Krug, K., Carpy, A., Behrends, G., Matic, K., Soares, N.C. and Macek, B. (2013). Deep coverage of the Escherichia coli proteome enables the assessment of false discovery rates in simple proteogenomic experiments. Mol. Cell. Proteomics 12, 3420-3430.

Kuhn, M.L., Zemaitaitis, B., Hu, L.I., Sahu, A., Sorensen, D., Minasov, G., Lima, B.P., Scholle, M., Mrksich, M., Anderson, W.F., Gibson, B.W., Schilling, B. and Wolfe A.J. (2014). Structural, kinetic and proteomic characterization of acetyl phosphate-dependent bacterial protein acetylation. PLoS One 9:e94816.

Kyte, J. and Doolittle, R.F. (1982). A simple method for displaying the hydropathic character of a protein. J. Mol. Biol. 157, 105-132.

Laganowsky, A., Reading, E., Hopper, J.T. and Robinson, C.V. (2013). Mass spectrometry of intact membrane protein complexes. Nat. Protoc. 8, 639-651.

Laganowsky, A., Reading, E., Allison, T.M., Ulmschneider, M.B., Degiacomi, M.T., Baldwin, A.J. and Robinson, C.V. (2014). Membrane proteins bind lipids selectively to modulate their structure and function. Nature 510, 172-175.

Lai, E.M., Nair, U., Phadke, N.D. and Maddock, J.R. (2004). Proteomic screening and identification of differentially distributed membrane proteins in Escherichia coli. Mol. Microbiol. 52, 1029-1044.

Lam, H.T., Antonioli, P., Righetti, P.G., Citterio, A. and Girault, H. (2007). Gel-free IEF in a membranesealed multicompartment cell for proteome prefractionation. Electrophoresis 28, 1860-1866.

Lancaster, W.A., Praissman, .JL., Poole, F.L. 2nd, Cvetkovic, A., Menon, A.L., Scott, J.W., Jenney, F.E. Jr., Thorgersen, M.P., Kalisiak, E., Apon, J.V., Trauger, S.A., Siuzdak, G., Tainer, J.A. and Adams, M.W. (2011). A computational framework for proteome-wide pursuit and prediction of metalloproteins using ICP-MS and MS/MS data. BMC Bioinformatics 12:64.

Lanciano, P., Vergnes, A., Grimaldi, S., Guigliarelli, B. and Magalon, A. (2007). Biogenesis of a respiratory complex is orchestrated by a single accessory protein. J. Biol. Chem. 282, 17468-17474.

Land, M., Hauser, L., Jun, S.R., Nookaew, I., Leuze, M.R., Ahn, T.H., Karpinets, T., Lund, O., Kora, G., Wassenaar, T., Poude,l S. and Ussery, D.W. (2015). Insights from 20 years of bacterial genome sequencing. Funct. Integr. Genomics 15, 141-161.

Landreh, M. and Robinson, C.V. (2015). A new window into the molecular physiology of membrane proteins. J. Physiol. 93, 355-362.

Lanouette, S., Mongeon, V., Figeys, D. and Couture, J.F. (2014). The functional diversity of protein lysine methylation. Mol. Syst. Biol. 10:724.

Lanucara, F., Holman, S.W., Gray, C.J. and Eyers, C.E. (2014). The power of ion mobility-mass spectrometry for structural characterization and the study of conformational dynamics. Nat. Chem. 6, 281-294.

Lapuente-Brun, E., Moreno-Loshuertos, R., Acín-Pérez, R., Latorre-Pellicer, A., Colás, C., Balsa, E., Perales-Clemente, E., Quirós, P.M., Calvo, E., Rodríguez-Hernández, M.A., Navas, P., Cruz, R., Carracedo, Á., López-Otín, C., Pérez-Martos, A., Fernández-Silva, P., Fernández-Vizarra, E. and 
Enríquez, J.A. (2013). Supercomplex assembly determines electron flux in the mitochondrial electron transport chain. Science 340, 1567-1570.

Larbi, N.B. and Jefferies, C. (2009). 2D-DIGE: comparative proteomics of cellular signalling pathways. Methods Mol Biol. 517, 105-32.

Lasker, K., Förster, F., Bohn, S., Walzthoeni, T., Villa, E., Unverdorben, P., Beck, F., Aebersold, R., Sali, A. and Baumeister, W. (2012). Molecular architecture of the 26S proteasome holocomplex determined by an integrative approach. Proc. Natl. Acad. Sci. U S A. 109, 1380-1387.

Lasserre, J.P., Beyne, E., Pyndiah, S., Lapaillerie, D., Claverol, S., Bonneu, M. (2006). A complexomic study of Escherichia coli using two-dimensional blue native/SDS polyacrylamide gel electrophoresis. Electrophoresis 27, 3306-21.

Leary, S.C. (2012). Blue native polyacrylamide gel electrophoresis: a powerful diagnostic tool for the detection of assembly defects in the enzyme complexes of oxidative phosphorylation. Methods Mol. Biol. 837, 195-206.

LeDuc, R.D., Taylor, G.K., Kim, Y.B., Januszyk, T.E., Bynum, L.H., Sola, J.V., Garavelli, J.S. and Kelleher, N.L. (2004). ProSight PTM: an integrated environment for protein identification and characterization by top-down mass spectrometry. Nucleic Acids Res. 32, W340-345.

Lee, A.G. (2003).Lipid-protein interactions in biological membranes: a structural perspective. Biochim. Biophys. Acta 1612, 1-40.

Lee, A.M., Sevinsky, J.R., Bundy, J.L., Grunden, A.M. and Stephenson, J.L. Jr. (2009). Proteomics of Pyrococcus furiosus, a hyperthermophilic archaeon refractory to traditional methods. J. Proteome Res. 8, 3844-3851.

Lee, D.W., Kim, D., Lee, Y.J., Kim, J.A., Choi, J.Y., Kang, S.and Pan, J.G. (2013). Proteomic analysis of acetylation in thermophilic Geobacillus kaustophilus. Proteomics 13, 2278-2282.

Leichert, L.I. and Jakob, U. (2004). Protein thiol modifications visualized in vivo. PLoS Biol. 2:e333.

Leichert, L.I. and Jakob, U. (2006). Global methods to monitor the thiol-disulfide state of proteins in vivo. Antioxid. Redox Signal. 8, 763-772.

Leitner, A., Walzthoeni, T., Kahraman, A., Herzog, F., Rinner, O., Beck, M. and Aebersold, R. (2010). Probing native protein structures by chemical cross-linking, mass spectrometry, and bioinformatics. Mol. Cell. Proteomics 9, 1634-1649.

Leitner, A., Joachimiak, L.A., Unverdorben, P., Walzthoeni, T., Frydman, J., Förster, F. and Aebersold, R. (2014). Chemical cross-linking/mass spectrometry targeting acidic residues in proteins and protein complexes. Proc. Natl. Acad. Sci. U S A 111, 9455-9460.

Lemaire, C. and Dujardin, G. (2008). Preparation of respiratory chain complexes from Saccharomyces cerevisiae wild-type and mutant mitochondria : activity measurement and subunit composition analysis. Methods Mol. Biol. 432, 65-81.

Lenaz, G. and Genova, M.L. (2009). Mobility and function of coenzyme Q (ubiquinone) in the mitochondrial respiratory chain. Biochim. Biophys. Acta 1787, 563-573.

Lenn, T., Leake, M.C. and Mullineaux, C.W. (2008). Clustering and dynamics of cytochrome $b d-\mathrm{I}$ complexes in the Escherichia coli plasma membrane in vivo. Mol. Microbiol. 70, 1397-1407.

Lenn, T. and Leake, M.C. (2015). Single-molecule studies of the dynamics and interactions of bacterial OXPHOS complexes. Biochim. Biophys. Acta pii: S0005-2728(15)00215-7. doi: 10.1016/j.bbabio.2015.10.008. [Epub ahead of print].

Leversen, N.A., de Souza, G.A., Målen, H., Prasad, S., Jonassen, I. and Wiker, H.G. (2009). Evaluation of signal peptide prediction algorithms for identification of mycobacterial signal peptides using sequence data from proteomic methods. Microbiology 155, 2375-2383.

Liao, G., Xie, L., Li, X., Cheng, Z. and Xie, J. (2014). Unexpected extensive lysine acetylation in the trump-card antibiotic producer Streptomyces roseosporus revealed by proteome-wide profiling. $J$. Proteomics 106, 260-269.

Lin, M.H., Hsu, T.L., Lin, S.Y., Pan, Y.J., Jan, J.T., Wang, J.T., Khoo, K.H. and Wu, S.H. (2009). Phosphoproteomics of Klebsiella pneumoniae NTUH-K2044 reveals a tight link between tyrosine phosphorylation and virulence. Mol. Cell. Proteomics. 8, 2613-2623.

Lin, M.H., Sugiyama, N. and Ishihama, Y. (2015). Systematic profiling of the bacterial phosphoproteome reveals bacterium-specific features of phosphorylation. Sci Signal. 8:rs10.

Lindahl, M., Mata-Cabana, A. and Kieselbach, T. (2011). The disulfide proteome and other reactive cysteine proteomes: analysis and functional significance. Antioxid. Redox Signal. 14, 2581-2642. 
Lindemann, C. and Leichert, L.I. (2012). Quantitative redox proteomics: the NOxICAT method. Methods Mol. Biol. 893, 387-403.

Lindemann, C., Lupilova, N., Müller, A., Warscheid, B., Meyer, H.E., Kuhlmann, K., Eisenacher, M. and Leichert, L.I. (2013). Redox proteomics uncovers peroxynitrite-sensitive proteins that help Escherichia coli to overcome nitrosative stress. J. Biol. Chem. 288, 19698-19714.

Listinsky, J.J., Siegal, G.P. and Listinsky, C.M. (2013). Glycoengineering in cancer therapeutics: a review with fucose-depleted trastuzumab as the model. Anticancer Drugs 24, 219-227.

Liu, F., Yang, M., Wang, X., Yang, S., Gu, J., Zhou, J., Zhang, X.E., Deng, J. and Ge, F. (2014). Acetylome analysis reveals diverse functions of lysine acetylation in Mycobacterium tuberculosis. Mol. Cell. Proteomics. 13, 3352-3366.

Liu, T., Tian, C.F. and Chen, W.X. (2015). Site-Specific Ser/Thr/Tyr Phosphoproteome of Sinorhizobium meliloti at Stationary Phase. PLoS One 10:e0139143.

Liu, X., Gong, X., Hicks, D.B., Krulwich, T.A., Yu, L. and Yu, C.A. (2007). Interaction between cytochrome $\mathrm{caa}_{3}$ and $\mathrm{F}_{1} \mathrm{~F}_{0}$-ATP synthase of alkaliphilic Bacillus pseudofirmus OF4 is demonstrated by saturation transfer electron paramagnetic resonance and differential scanning calorimetry assays. Biochemistry 46, 306-313.

Liu, X., Sirotkin, Y., Shen, Y., Anderson, G., Tsai, Y.S., Ting, Y.S., Goodlett, D.R., Smith, R.D., Bafna, V. and Pevzner, P.A. (2012). Protein identification using top-down. Mol. Cell. Proteomics 11, M111008524.

Llorente-Garcia, I., Lenn, T., Erhardt, H., Harriman, O.L., Liu, L.N., Robson, A., Chiu, S.W., Matthews, S., Willis, N.J., Bray, C.D., Lee, S.H., Shin, J.Y., Bustamante, C., Liphardt, J., Friedrich, T., Mullineaux, C.W. and Leake, M.C. (2014). Single-molecule in vivo imaging of bacterial respiratory complexes indicates delocalized oxidative phosphorylation. Biochim. Biophys. Acta 1837, 811-824.

Loi, V.V., Rossius, M. and Antelmann, H. (2015). Redox regulation by reversible protein $S$-thiolation in bacteria. Front. Microbiol. 6:187.

Loll, P.J. (2014). Membrane proteins, detergents and crystals: what is the state of the art? Acta Crystallogr F Struct Biol Commun. 70, 1576-1583.

Lopez-Campistrous, A., Semchuk, P., Burke, L., Palmer-Stone, T., Brokx, S.J., Broderick, G., Bottorff, D., Bolch, S., Weiner, J.H. and Ellison, M.J. (2005). Localization, annotation, and comparison of the Escherichia coli K-12 proteome under two states of growth. Mol. Cell. Proteomics. 4, 12051209.

Lorenzi, M., Sylvi, L., Gerbaud, G., Mileo, E., Halgand, F., Walburger, A., Vezin, H., Belle, V., Guigliarelli, B. and Magalon, A. (2012). Conformational selection underlies recognition of a molybdoenzyme by its dedicated chaperone. PLoS One 7:e49523.

Lu, B., McClatchy, D.B., Kim, J.Y., Yates, J.R. 3rd. (2008). Strategies for shotgun identification of integral membrane proteins by tandem mass spectrometry. Proteomics 8, 3947-3955.

Luchini, A., Espina, V. and Liotta, L.A. (2014). Protein painting reveals solvent-excluded drug targets hidden within native protein-protein interfaces. Nat. Commun. 5:4413.

Luirink, J., Yu, Z., Wagner, S. and de Gier, J.W. (2012). Biogenesis of inner membrane proteins in Escherichia coli. Biochim. Biophys. Acta 1817, 965-976.

Lundgren, D.H., Hwang, S.I., Wu, L. and Han, D.K. (201). Role of spectral counting in quantitative proteomics. Expert Rev. Proteomics 7, 39-53.

Lutter, P., Meyer, H.E., Langer, M., Witthohn, K., Dormeyer, W., Sickmann, A. and Blüggel, M. (2001). Investigation of charge variants of rViscumin by two-dimensional gel electrophoresis and mass spectrometry. Electrophoresis 22, 2888-2897.

Ma, B. (2015). Novor: Real-Time Peptide de Novo Sequencing Software. J. Am. Soc. Mass Spectrom. 26, 1885-1894.

Maas, M.F., Krause, F., Dencher, N.A. and Sainsard-Chanet, A. (2009). Respiratory complexes III and IV are not essential for the assembly/stability of complex I in fungi. J. Mol. Biol. 387, 259-269.

Macek, B., Mijakovic, I., Olsen, J.V., Gnad, F., Kumar, C., Jensen, P.R. and Mann, M. (2007). The serine/threonine/tyrosine phosphoproteome of the model bacterium Bacillus subtilis. Mol. Cell. Proteomics 6, 697-707.

Macek, B., Gnad, F., Soufi, B., Kumar, C., Olsen, J.V., Mijakovic, I. and Mann, M. (2008). Phosphoproteome analysis of E. coli reveals evolutionary conservation of bacterial Ser/Thr/Tyr phosphorylation. Mol. Cell. Proteomics 7, 299-307. 
Macek, B., Mann, M. and Olsen, J.V. (2009). Global and site-specific quantitative phosphoproteomics: principles and applications. Annu. Rev. Pharmacol. Toxicol. 49, 199-221.

Macek, B. and Mijakovic, I. (2011). Site-specific analysis of bacterial phosphoproteomes. Proteomics 11, 3002-3011.

Maclean, B., Tomazela, D.M., Abbatiello, S.E., Zhang, S., Whiteaker, J.R., Paulovich, A.G., Carr, S.A. and Maccoss, M.J. (2010a). Effect of collision energy optimization on the measurement of peptides by selected reaction monitoring (SRM) mass spectrometry. Anal. Chem. 82, 10116-10124.

MacLean, B., Tomazela, D M., Shulman, N., Chambers, M., Finney, G.L., Frewen, B., Kern, R., Tabb, D. L., Liebler, D.C. and MacCoss, M.J. (2010b). Skyline: an open source document editor for creating and analyzing targeted proteomics experiments. Bioinformatics 26, 966-968.

Maddalo, G., Chovanec, P., Stenberg-Bruzell, F., Nielsen, H.V., Jensen-Seaman, M.I., Ilag, L.L., Kline, K.A. and Daley, D.O. (2011). A reference map of the membrane proteome of Enterococcus faecalis. Proteomics 11, 3935-3941.

Magalon, A. and Alberge, F. (2015). Distribution and dynamics of OXPHOS complexes in the bacterial cytoplasmic membrane. Biochim. Biophys. Acta pii: S0005-2728(15)00222-4. doi: 10.1016/j.bbabio.2015.10.015. [Epub ahead of print].

Magalon, A. and Mendel, R.R. (2015). Biosynthesis and Insertion of the Molybdenum Cofactor. Ecosal Plus 6(2). doi: 10.1128/ecosalplus.ESP-0006-2013.

Makarov, A. (2000). Electrostatic axially harmonic orbital trapping: a high-performance technique of mass analysis. Anal. Chem. 72, 1156-1162.

Målen, H., Berven, F.S., Søfteland, T., Arntzen, M.Ø., D'Santos, C.S., De Souza, G.A. and Wiker, H.G. (2008). Membrane and membrane-associated proteins in Triton X-114 extracts of Mycobacterium bovis BCG identified using a combination of gel-based and gel-free fractionation strategies. Proteomics 8, 1859-1870.

Mamyrin, B.A. (2001). Time-of-flight mass spectrometry (concepts, achievements, and prospects). Int. J. Mass Spectrom. 206, 251-266.

Mandell, J.G., Baerga-Ortiz, A., Croy, C.H., Falick, A.M. and Komives, E.A. (2005a). Application of amide proton exchange mass spectrometry for the study of protein-protein interactions. Curr. Protoc. Protein. Sci. Chapter 20:Unit20.9.

Mandell, J.G., Baerga-Ortiz, A., Falick, A.M. and Komives, E.A. (2005b). Measurement of solvent accessibility at protein-protein interfaces. Methods Mol. Biol. 305, 65-80.

Maranzana, E., Barbero, G., Falasca, A.I., Lenaz, G. and Genova, M.L. (2013). Mitochondrial respiratory supercomplex association limits production of reactive oxygen species from complex I. Antioxid. Redox Signal. 19, 1469-1480.

March, R.E. (1997). An Introduction to Quadrupole Ion Trap Mass Spectrometry. J. Mass Spectrom. 32, 351-369.

Marshall, A.G., Hendrickson, C.L. and Jackson, G.S. (1998). Fourier transform ion cyclotron resonance mass spectrometry: a primer. Mass Spectrom. Rev. 17, 1-35.

Marcoux, J. and Cianférani, S. (2015). Towards integrative structural mass spectrometry: Benefits from hybrid approaches. Methods 89, 4-12.

Maret, W. (2010). Metalloproteomics, metalloproteomes, and the annotation of metalloproteins. Metallomics 2, 117-125.

Marín-Buera, L., García-Bartolomé, A., Morán, M., López-Bernardo, E., Cadenas, S., Hidalgo, B., Sánchez, R., Seneca, S., Arenas, J., Martín, M.A. and Ugalde, C. (2015). Differential proteomic profiling unveils new molecular mechanisms associated with mitochondrial complex III deficiency. J. Proteomics 113, 38-56.

Marozava, S., Röling, W.F., Seifert, J., Küffner, R., von Bergen, M. and Meckenstock, R.U. (2014). Physiology of Geobacter metallireducens under excess and limitation of electron donors. Part I. Batch cultivation with excess of carbon sources. Syst. Appl. Microbiol. 37, 277-286.

Marques, I., Dencher, N.A., Videira, A. and Krause, F. (2007). Supramolecular organization of the respiratory chain in Neurospora crassa mitochondria. Eukaryot. Cell 6, 2391-2405.

Martfeld, A.N., Rajagopalan, V., Greathouse, D.V. and Koeppe, R.E. 2nd. (2015). Dynamic regulation of lipid-protein interactions. Biochim. Biophys. Acta 1848, 1849-1859.

Masuda, T., Saito, N., Tomita, M. and Ishihama, Y. (2009). Unbiased quantitation of Escherichia coli membrane proteome using phase transfer surfactants. Mol. Cell. Proteomics. 8, 2770-2777. 
Mathy, G. and Sluse, F.E. (2008). Mitochondrial comparative proteomics: strengths and pitfalls. Biochim. Biophys. Acta 1777, 1072-1077.

Matias, P.M., Pereira, I.A., Soares, C.M. and Carrondo, M.A. (2005). Sulphate respiration from hydrogen in Desulfovibrio bacteria: a structural biology overview. Prog. Biophys. Mol. Biol. 89, 292-329.

Matsumoto, K., Hara, H., Fishov, I., Mileykovskaya, E. and Norris, V. (2015). The membrane: transertion as an organizing principle in membrane heterogeneity. Front. Microbiol. 6:572.

Matus-Ortega, M.G., Cárdenas-Monroy, C.A., Flores-Herrera, O., Mendoza-Hernández, G., Miranda, M., González-Pedrajo, B., Vázquez-Meza, H. and Pardo, J.P. (2015). New complexes containing the internal alternative NADH dehydrogenase (Ndi1) in mitochondria of Saccharomyces cerevisiae. Yeast 32, 629-641.

Mawuenyega, K.G., Forst, C.V., Dobos, K.M., Belisle. J.T., Chen, J., Bradbury, E.M., Bradbury, A.R. and Chen, X. (2005). Mycobacterium tuberculosis functional network analysis by global subcellular protein profiling. Mol. Biol. Cell 16, 396-404.

May, J.C. and McLean, J.A. (2015). Ion mobility-mass spectrometry: time-dispersive instrumentation. Anal. Chem. 87, 1422-1436.

McAlister, G.C., Huttlin, E.L., Haas, W., Ting, L., Jedrychowski, M.P., Rogers, J.C., Kuhn, K., Pike, I., Grothe, R.A., Blethrow, J.D. and Gygi, S.P. (2012). Increasing the multiplexing capacity of TMTs using reporter ion isotopologues with isobaric masses. Anal. Chem. 84, 7469-7478.

McDonald, C.A., Yang, J.Y., Marathe, V., Yen, T.Y. and Macher, B.A. (2009). Combining results from lectin affinity chromatography and glycocapture approaches substantially improves the coverage of the glycoproteome. Mol. Cell. Proteomics 8, 287-301.

McKenzie, M., Lazarou, M., Thorburn, D.R., Ryan, M.T. (2007). Analysis of mitochondrial subunit assembly into respiratory chain complexes using Blue Native polyacrylamide gel electrophoresis. Anal. Biochem. 364, 128-137.

McLuckey, S.A., Van Berkel, G.J., Goeringer, D.E. and Glish, G.L. (1994). Ion trap mass spectrometry. Using high-pressure ionization. Anal. Chem. 66, 737A-743A.

Melo, A.M. and Teixeira, M. (2015). Supramolecular organization of bacterial aerobic respiratory chains: From cells and back. Biochim. Biophys. Acta. pii: S0005-2728(15)00224-8. doi: 10.1016/j.bbabio.2015.11.001. [Epub ahead of print].

Méndez-García, C., Peláez, A.I., Mesa, V., Sánchez, J., Golyshina, O.V. and Ferrer, M. (2015). Microbial diversity and metabolic networks in acid mine drainage habitats. Front. Microbiol. 6:475.

Menon, A.L., Poole, F.L. 2nd., Cvetkovic, A., Trauger, S.A., Kalisiak, E., Scott, J.W., Shanmukh, S., Praissman, J., Jenney, F.E. Jr., Wikoff, W.R., Apon, J.V., Siuzdak, G. and Adams, M.W. (2009). Novel multiprotein complexes identified in the hyperthermophilic archaeon Pyrococcus furiosus by non-denaturing fractionation of the native proteome. Mol. Cell. Proteomics 8, 735-751.

Meyer, K. and Selbach, M. (2015). Quantitative affinity purification mass spectrometry: a versatile technology to study protein-protein interactions. Front. Genet. 6:237.

Mijakovic, I. and Macek, B. (2012). Impact of phosphoproteomics on studies of bacterial physiology. FEMS Microbiol Rev. 36, 877-892.

Mijakovic, I. and Deutscher, J. (2015). Protein-tyrosine phosphorylation in Bacillus subtilis: a 10-year retrospective. Front. Microbiol. 6:18.

Mikkat, S., Fulda, S. and Hagemann, M. (2014). A 2D gel electrophoresis-based snapshot of the phosphoproteome in the cyanobacterium Synechocystis sp. strain PCC 6803. Microbiology 160, 296-306.

Mileykovskaya, E. and Dowhan, W. (2014). Cardiolipin-dependent formation of mitochondria respiratory supercomplexes. Chem. Phys. Lipids 179, $42-48$.

Millea, K.M., Krull, I.S., Cohen, S.A., Gebler, J.C. and Berger, S.J. (2006). Integration of multidimensional chromatographic protein separations with a combined "top-down" and "bottomup" proteomic strategy. J. Proteome Res. 5, 135-146.

Mimaki, M., Wang, X., McKenzie, M., Thorburn, D.R. and Ryan, M.T. (2012). Understanding mitochondrial complex I assembly in health and disease. Biochim. Biophys. Acta 1817, 851-862.

Minden, J.S. (2012). Two-dimensional difference gel electrophoresis. Methods Mol. Biol. 869, 287-304. 
Mitsopoulos, P., Chang, Y.H., Wai, T., König, T., Dunn, S.D., Langer, T. and Madrenas, J. (2015). Stomatin-like protein 2 is required for in vivo mitochondrial respiratory chain supercomplex formation and optimal cell function. Mol. Cell Biol. 35, 1838-1847.

Molloy, M.P. (2008). Isolation of bacterial cell membranes proteins using carbonate extraction. Methods Mol. Biol. 424, 397-401.

Moore, K.E., Carlson, S.M., Camp, N.D., Cheung, P., James, R.G., Chua, K.F., Wolf-Yadlin, A. and Gozani, O. (2013). A general molecular affinity strategy for global detection and proteomic analysis of lysine methylation. Mol. Cell 50, 444-456.

Moraes, I., Evans, G., Sanchez-Weatherby, J., Newstead, S. and Stewart, P.D. (2014). Membrane protein structure determination - the next generation. Biochim. Biophys. Acta 1838, 78-87.

Moreno-Beltrán, B., Díaz-Moreno, I., González-Arzola, K., Guerra-Castellano, A., Velázquez-Campoy, A., De la Rosa, M.A. and Díaz-Quintana, A. (2015). Respiratory complexes III and IV can each bind two molecules of cytochrome $c$ at low ionic strength. FEBS Lett. 589, 476-483.

Morgner, N., Montenegro, F., Barrera, N.P. and Robinson, C.V. (2012). Mass spectrometry-from peripheral proteins to membrane motors. J. Mol. Biol. 423, 1-13.

Muster, B., Kohl, W., Wittig, I., Strecker, V., Joos, F., Haase, W., Bereiter-Hahn, J. and Busch, K. (2010). Respiratory chain complexes in dynamic mitochondria display a patchy distribution in life cells. PLoS One 5:e11910.

Nag, B., Arimilli, S., Koukis, B., Rhodes, E., Baichwal, V., Sharma, S.D. (1994). Intramolecular charge heterogeneity in purified major histocompatibility class II alpha and beta polypeptide chains. $J$. Biol. Chem. 269, 10061-10070.

Nambi, S., Gupta, K., Bhattacharyya, M., Ramakrishnan, P., Ravikumar, V., Siddiqui, N., Thomas, A.T., Visweswariah, S.S. Cyclic AMP-dependent protein lysine acylation in mycobacteria regulates fatty acid and propionate metabolism. J. Biol. Chem. 288, 14114-14124.

Neidhardt, F.C. (2011). How microbial proteomics got started. Proteomics 11, 2943-2946.

Neumann, S., Wessels, H.J., Rijpstra, W.I., Sinninghe Damsté, J.S., Kartal, B., Jetten, M.S. and van Niftrik, L. (2014). Isolation and characterization of a prokaryotic cell organelle from the anammox bacterium Kuenenia stuttgartiensis. Mol. Microbiol. 94, 794-802.

Nicke, T., Schnitzer, T., Münch, K., Adamczack, J., Haufschildt, K., Buchmeier, S., Kucklick, M., Felgenträger, U., Jänsch, L., Riedel, K. and Layer, G. (2013). Maturation of the cytochrome $c d_{1}$ nitrite reductase NirS from Pseudomonas aeruginosa requires transient interactions between the three proteins NirS, NirN and NirF. Biosci. Rep. 33, pii: e00048.

Niebisch, A. and Bott, M. (2003). Purification of a cytochrome $b c-a a_{3}$ supercomplex with quinol oxidase activity from Corynebacterium glutamicum. Identification of a fourth subunity of cytochrome $a a_{3}$ oxidase and mutational analysis of diheme cytochrome $c_{1}$. J. Biol. Chem. 278, 4339-4346.

Niederman, R.A. (2013). Membrane development in purple photosynthetic bacteria in response to alterations in light intensity and oxygen tension. Photosynth. Res. 116, 333-348.

Nijtmans, L.G., Huynen, M.A. and Thorburn, D.R. (2013). Mutations in the UQCC1-interacting protein, UQCC2, cause human complex III deficiency associated with perturbed cytochrome $b$ protein expression. PLoS Genet. 9:e1004034.

Niu, S. and Ruotolo, B.T. (2015). Collisional unfolding of multiprotein complexes reveals cooperative stabilization upon ligand binding. Protein Sci. 24, 1272-1281.

Nothwang, H.G. and Schindler, J. (2009). Two-dimensional separation of membrane proteins by 16BAC-SDS-PAGE. Methods Mol. Biol. 528, 269-277.

Nouws, J., Nijtmans, L., Houten, S.M., van den Brand, M., Huynen, M., Venselaar, H., Hoefs, S., Gloerich, J., Kronick, J., Hutchin, T., Willems, P., Rodenburg, R., Wanders, R., van den Heuvel, L., Smeitink, J. and Vogel, R.O. (2010). Acyl-CoA dehydrogenase 9 is required for the biogenesis of oxidative phosphorylation complex I. Cell Metab. 12, 283-294.

Nowak, G. and Bakajsova, D. (2015). Protein kinase C- $\alpha$ interaction with $F_{0} F_{1}$-ATPase promotes $F_{0} F_{1}$ ATPase activity and reduces energy deficits in injured renal cells. J. Biol. Chem. 290, 7054-7066.

Nübel, E., Wittig, I., Kerscher, S., Brandt, U. and Schägger, H. (2009). Two-dimensional native electrophoretic analysis of respiratory supercomplexes from Yarrowia lipolytica. Proteomics 9, 2408-2418.

Nyon, M.P., Prentice, T., Day, J., Kirkpatrick, J., Sivalingam, G.N., Levy, G., Haq, I., Irving, J.A., Lomas, D.A., Christodoulou, J., Gooptu, B. and Thalassinos, K. (2015). An integrative approach 
combining ion mobility mass spectrometry, X-ray crystallography, and nuclear magnetic resonance spectroscopy to study the conformational dynamics of $\alpha 1$-antitrypsin upon ligand binding. Protein Sci. 24, 1301-1312.

O'Farrell, P.H. (1975). High resolution two-dimensional electrophoresis of proteins. J. Biol. Chem. 250, 4007-4021.

Okanishi, H., Kim, K., Masui, R., Kuramitsu, S. (2013). Acetylome with structural mapping reveals the significance of lysine acetylation in Thermus thermophilus. J. Proteome Res. 12, 3952-3968.

Oliveira, A.P. and Picotti, P. (2014). Global analysis of protein structural changes in complex proteomes. Nat. Biotechnol. 32, 1036-1044.

Oliveira, B.M., Coorssen, J.R. and Martins-de-Souza, D. (2014). 2DE: the phoenix of proteomics. J. Proteomics 104, 140-50.

Oliver, P.M., Crooks, J.A., Leidl, M., Yoon, E.J., Saghatelian, A. and Weibel, D.B. (2014). Localization of anionic phospholipids in Escherichia coli cells. J. Bacteriol. 196, 3386-3398.

Olsen, J.V. and Macek, B. (2009). High accuracy mass spectrometry in large-scale analysis of protein phosphorylation. Methods Mol. Biol. 492, 131-42.

Olsen, J.V. and Mann, M. (2013). Status of large-scale analysis of post-translational modifications by mass spectrometry. Mol. Cell. Proteomics. 12, 3444-3452.

Ong, S.E., Blagoev, B., Kratchmarova, I., Kristensen, D. B., Steen, H., Pandey, A. and Mann, M. (2002). Stable isotope labeling by amino acids in cell culture, SILAC, as a simple and accurate approach to expression proteomics. Mol. Cell. Proteomics 1, 376-386.

Orellana, R., Hixson, K.K., Murphy, S., Mester, T., Sharma, M.L., Lipton, M.S. and Lovley, D.R. (2014). Proteome of Geobacter sulfurreducens in the presence of U(VI). Microbiology 160, 26072617.

Oren, A. and Garrity, G.M. (2014). Then and now: a systematic review of the systematics of prokaryotes in the last 80 years. Antonie Van Leeuwenhoek 106, 43-56.

Osborne, B., Cooney, G.J. and Turner, N. (2014). Are sirtuin deacylase enzymes important modulators of mitochondrial energy metabolism? Biochim. Biophys. Acta 1840, 1295-1302.

Owens, R. (2011). Methods in structural proteomics. Methods 55, 1-2.

Paetzel, M. (2014). Structure and mechanism of Escherichia coli type I signal peptidase. Biochim. Biophys. Acta 1843, 1497-1508.

Pagliarini, D.J. and Dixon, J.E. (2006). Mitochondrial modulation: reversible phosphorylation takes center stage? Trends Biochem. Sci. 31, 26-34.

Palmisano, G., Sardanelli, A.M., Signorile, A., Papa, S. and Larsen, M.R. (2007). The phosphorylation pattern of bovine heart complex I subunits. Proteomics 7, 1575-1583.

Pan, J., Ye, Z., Cheng, Z., Peng, X., Wen, L. and Zhao, F. (2014). Systematic analysis of the lysine acetylome in Vibrio parahemolyticus. J. Proteome Res. 13, 3294-3302.

Pang, C.N., Gasteiger, E. and Wilkins, M.R. (2010). Identification of arginine- and lysine-methylation in the proteome of Saccharomyces cerevisiae and its functional implications. BMC Genomics 11:92.

Papanicolaou, K.N., O'Rourke, B. and Foster, D.B. (2014). Metabolism leaves its mark on the powerhouse: recent progress in post-translational modifications of lysine in mitochondria. Front. Physiol. 5:301.

Pappin, D.J., Hojrup, P. and Bleasby, A.J. (1993). Rapid identification of proteins by peptide-mass fingerprinting. Curr. Biol. 3, 327-332.

Paradies, G., Paradies, V., De Benedictis, V., Ruggiero, F.M. and Petrosillo, G. (2014). Functional role of cardiolipin in mitochondrial bioenergetics. Biochim. Biophys. Acta 1837, 408-417.

Parker, J.L., Jones, A.M., Serazetdinova, L., Saalbach, G., Bibb, M.J. and Naldrett, M.J. (2010). Analysis of the phosphoproteome of the multicellular bacterium Streptomyces coelicolor A3(2) by protein/peptide fractionation, phosphopeptide enrichment and high-accuracy mass spectrometry. Proteomics 10, 2486-97.

Paton, L.N., Gerrard, J.A. and Bryson, W.G. (2008). Investigations into charge heterogeneity of wool intermediate filament proteins. J. Proteomics. 71, 513-29.

Pedrioli, P.G. (2010). Trans-proteomic pipeline: a pipeline for proteomic analysis. Methods Mol. Biol. 604, 213-238. 
Pereira, S.F., Goss, L. and Dworkin, J. (2011). Eukaryote-like serine/threonine kinases and phosphatases in bacteria. Microbiol. Mol. Biol. Rev. 75, 192-212.

Perkins, D.N., Pappin, D.J., Creasy, D.M. and Cottrell, J.S. (1999). Probability-based protein identification by searching sequence databases using mass spectrometry data. Electrophoresis 20 , 3551-3567.

Perry, R.H., Cooks, R.G. and Noll, R.J. (2008). Orbitrap mass spectrometry: instrumentation, ion motion and applications. Mass Spectrom. Rev. 27, 661-699.

Peterman, S.M., Dufresne, C.P. and Horning, S. (2005). The use of a hybrid linear trap/FT-ICR mass spectrometer for on-line high resolution/high mass accuracy bottom-up sequencing. J. Biomol. Tech. 16, 112-124.

Peters, J.W., Schut, G.J., Boyd, E.S., Mulder, D.W., Shepard, E.M., Broderick, J.B., King, P.W. and Adams, M.W. (2015). [FeFe]- and [NiFe]-hydrogenase diversity, mechanism, and maturation. Biochim. Biophys. Acta 1853, 1350-1369.

Petrotchenko, E.V. and Borchers, C.H. (2010). Crosslinking combined with mass spectrometry for structural proteomics. Mass Spectrom. Rev. 29, 862-876.

Petrotchenko, E.V. and Borchers, C.H. (2014). Modern mass spectrometry-based structural proteomics. Adv. Protein Chem. Struct. Biol. 95, 193-213.

Pevtsov, S., Fedulova, I., Mirzaei, H., Buck, C. and Zhang, X. (2006). Performance evaluation of existing de novo sequencing algorithms. J. Proteome Res. 5, 3018-3028.

Phillips, N.J., Steichen, C.T., Schilling, B., Post, D.M., Niles, R.K., Bair, T.B., Falsetta, M.L., Apicella, M.A. and Gibson, B.W. (2012). Proteomic analysis of Neisseria gonorrhoeae biofilms shows shift to anaerobic respiration and changes in nutrient transport and outermembrane proteins. PLoS One 7:e38303.

Planas-Iglesias, J., Dwarakanath, H., Mohammadyani, D., Yanamala, N., Kagan, V.E. and KleinSeetharaman, J. (2015). Cardiolipin Interactions with Proteins. Biophys. J. 109, 1282-1294.

Pocsfalvi, G., Cuccurullo, M., Schlosser, G., Cacace, G., Siciliano, R.A., Mazzeo, M.F., Scacco, S., Cocco, T., Gnoni, A., Malorni, A. and Papa, S. (2006). Shotgun proteomics for the characterization of subunit composition of mitochondrial complex I. Biochim. Biophys. Acta 1757, 1438-1450.

Poetsch, A. and Wolters, D. (2008). Bacterial membrane proteomics. Proteomics 8, 4100-4122.

Pohl, S., Tu, W.Y., Aldridge, P.D., Gillespie, C., Hahne, H., Mäder, U., Read T.D. and Harwood, C.R. (2011). Combined proteomic and transcriptomic analysis of the response of Bacillus anthracis to oxidative stress. Proteomics 11, 3036-3055.

Poland, T., Rabilloud, T. and Sihna P. (2005). Silver staining of 2D gels. In J.M. Walker (Ed.), The proteomics protocols handbook, pp. 177-184. Totowa, N.J.: Human Press.

Ponomarova, O. and Patil, K.R. (2015). Metabolic interactions in microbial communities: untangling the Gordian knot. Curr. Opin. Microbiol. 27, 37-44.

Price, C.E. and Driessen, A.J. (2010). Biogenesis of membrane bound respiratory complexes in Escherichia coli. Biochim. Biophys. Acta 1803, 748-766.

Prime, T.A., Blaikie, F.H., Evans, C., Nadtochiy, S.M., James, A.M., Dahm, C.C., Vitturi, D.A., Patel, R.P., Hiley, C.R., Abakumova, I., Requejo, R., Chouchani, E.T., Hurd, T.R., Garvey, J.F., Taylor, C.T., Brookes, P.S., Smith, R.A. and Murphy, M.P. (2009). A mitochondria-targeted S-nitrosothiol modulates respiration, nitrosates thiols, and protects against ischemia-reperfusion injury. Proc. Natl. Acad. Sci. U S A 106, 10764-10769.

Prisic, S., Dankwa, S., Schwartz, D., Chou, M.F., Locasale, J.W., Kang, C.M., Bemis, G., Church, G.M., Steen, H. and Husson, R.N. (2010). Extensive phosphorylation with overlapping specificity by Mycobacterium tuberculosis serine/threonine protein kinases. Proc. Natl. Acad. Sci. U S A 107, 7521-7526.

Prunetti, L., Infossi, P., Brugna, M., Ebel, C., Giudici-Orticoni, M.T. and Guiral, M. (2010). New functional sulfide oxidase-oxygen reductase supercomplex in the membrane of the hyperthermophilic bacterium Aquifex aeolicus. J. Biol. Chem. 285, 41815-41826.

Puig, O., Caspary, F., Rigaut, G., Rutz, B., Bouveret, E., Bragado-Nilsson, E., Wilm, M. and Séraphin, B. (2001). The tandem affinity purification (TAP) method: a general procedure of protein complex purification. Methods 24, 218-229. 
Puts, C.F., Lenoir, G., Krijgsveld, J., Williamson, P. and Holthuis, J.C. (2010). A P4-ATPase protein interaction network reveals a link between aminophospholipid transport and phosphoinositide metabolism. J. Proteome Res. 9, 833-842.

Pyndiah, S., Lasserre, J.P., Ménard, A., Claverol, S., Prouzet-Mauléon, V., Mégraud, F., Zerbib, F. and Bonneu, M. (2007). Two-dimensional blue native/SDS gel electrophoresis of multiprotein complexes from Helicobacter pylori. Mol. Cell. Proteomics 6, 193-206.

Rabilloud, T. (2009). Membrane proteins and proteomics: love is possible, but so difficult. Electrophoresis 30, Suppl 1:S174-80.

Rabilloud, T., Chevallet, M., Luche, S. and Lelong, C. (2008a). Fully denaturing two-dimensional electrophoresis of membrane proteins: a critical update. Proteomics 8, 3965-3973.

Rabilloud, T., Chevallet, M., Luche, S. and Lelong, C. (2008b). Two-dimensional gel electrophoresis in proteomics: Past, present and future. J. Proteomics 73, 2064-2077.

Rabuck, J.N., Hyung, S.J., Ko, K.S., Fox, C.C., Soellner, M.B. and Ruotolo, B.T. (2013). Activation state-selective kinase inhibitor assay based on ion mobility-mass spectrometry. Anal. Chem. 85, 6995-7002.

Ramírez-Aguilar, S.J., Keuthe, M., Rocha, M., Fedyaev, V.V., Kramp, K., Gupta, K.J., Rasmusson, A.G., Schulze, W.X. and van Dongen, J.T. (2011). The composition of plant mitochondrial supercomplexes changes with oxygen availability. J. Biol. Chem. 286, 43045-43053.

Ramisetty, S.R. and Washburn, M.P. (2011). Unraveling the dynamics of protein interactions with quantitative mass spectrometry. Crit. Rev. Biochem. Mol. Biol. 46, 216-228.

Rand, K.D., Zehl, M. and Jørgensen, T.J. (2014). Measuring the hydrogen/deuterium exchange of proteins at high spatial resolution by mass spectrometry: overcoming gas-phase hydrogen/deuterium scrambling. Acc. Chem. Res. 47, 3018-3027.

Rappsilber, J. (2011). The beginning of a beautiful friendship: cross-linking/mass spectrometry and modelling of proteins and multi-protein complexes. J. Struct. Biol. 173, 530-540.

Rappsilber, J. and Mann, M. (2002). What does it mean to identify a protein in proteomics? Trends Biochem. Sci. 27, 74-78.

Rappsilber, J., Siniossoglou, S., Hurt, E.C. and Mann, M. (2000). A generic strategy to analyze the spatial organization of multi-protein complexes by cross-linking and mass spectrometry. Anal. Chem. 72, 267-275.

Ravichandran, A., Sugiyama, N., Tomita, M., Swarup, S. and Ishihama, Y. (2009). Ser/Thr/Tyr phosphoproteome analysis of pathogenic and non-pathogenic Pseudomonas species. Proteomics $\mathbf{9}$, 2764-2775.

Ravikumar, V., Shi, L., Krug, K., Derouiche, A., Jers, C., Cousin, C., Kobir, A., Mijakovic, I. and Macek, B. (2014). Quantitative phosphoproteome analysis of Bacillus subtilis reveals novel substrates of the kinase PrkC and phosphatase PrpC. Mol. Cell. Proteomics. 13, 1965-1978.

Reiland, S., Messerli, G., Baerenfaller, K., Gerrits, B., Endler, A., Grossmann, J., Gruissem, W. and Baginsky, S. (2009). Large-scale Arabidopsis phosphoproteome profiling reveals novel chloroplast kinase substrates and phosphorylation networks. Plant Physiol. 150, 889-903.

Reinders. J., Wagner, K., Zahedi, R.P., Stojanovski, D., Eyrich, B., van der Laan, M., Rehling, P., Sickmann, A., Pfanner, N. and Meisinger, C. (2007). Profiling phosphoproteins of yeast mitochondria reveals a role of phosphorylation in assembly of the ATP synthase. Mol. Cell. Proteomics 6, 1896-1906.

Remmerie, N., De Vijlder, T., Valkenborg, D., Laukens, K., Smets, K., Vreeken, J., Mertens, I., Carpentier, S.C., Panis, B., De Jaeger, G., Blust, R., Prinsen, E. and Witters, E. (2011). Unraveling tobacco BY-2 protein complexes with BN PAGE/LC-MS/MS and clustering methods. $J$. Proteomics 74, 1201-1217.

Renier, S., Chafsey, I., Chambon, C., Caccia, N., Charbit, A., Hébraud, M. and Desvaux, M. (2015). Contribution of the multiple Type I signal peptidases to the secretome of Listeria monocytogenes: deciphering their specificity for secreted exoproteins by exoproteomic analysis. J. Proteomics 117, 95-105.

Rhein, V.F., Carroll, J., Ding, S., Fearnley, I.M. and Walker, J.E. (2013). NDUFAF7 methylates arginine 85 in the NDUFS2 subunit of human complex I. J. Biol. Chem. 288, 33016-33026.

Richter, K., Schicklberger, M., Gescher, J. (2012). Dissimilatory reduction of extracellular electron acceptors in anaerobic respiration. Appl. Environ. Microbiol. 78, 913-21. 
Rigaut, G., Shevchenko, A., Rutz, B., Wilm, M., Mann, M. and Séraphin, B. (1999). A generic protein purification method for protein complex characterization and proteome exploration. Nat Biotechnol. 17, 1030-1032.

Robinson, C., Matos, C.F., Beck, D., Ren, C., Lawrence, J., Vasisht, N. and Mendel, S. (2011). Transport and proofreading of proteins by the twin-arginine translocation (Tat) system in bacteria. Biochim. Biophys. Acta 1808, 876-884.

Robinson, N.J. and Winge, D.R. (2010). Copper metallochaperones. Annu. Rev. Biochem. 79, 537-562.

Robledo, V.R. and Smyth, W.F. (2014). Review of the CE-MS platform as a powerful alternative to conventional couplings in bio-omics and target-based applications. Electrophoresis 35, 2292-2308.

Rosca, M.G., Vazquez, E.J., Kerner, J., Parland, W., Chandler, M.P., Stanley, W., Sabbah, H.N. and Hoppel, C.L. (2008). Cardiac mitochondria in heart failure: decrease in respirasomes and oxidative phosphorylation. Cardiovasc. Res. 80, 30-39.

Roepstorff, P. and Fohlman, J. (1984). Proposal for a common nomenclature for sequence ions in mass spectra of peptides. Biomed. Mass Spectrom. 11, 601.

Ross, P. L., Huang, Y.N., Marchese, J.N., Williamson, B., Parker, K., Hattan, S., Khainovski, N., Pillai, S., Dey, S., Daniels, S., Purkayastha, S., Juhasz, P., Martin, S., Bartlet-Jones, M., He, F., Jacobson, A. and Pappin, D.J. (2004). Multiplexed protein quantitation in Saccharomyces cerevisiae using amine-reactive isobaric tagging reagents. Mol. Cell. Proteomics 3, 1154-1169.

Rybak, J.N., Scheurer, S.B., Neri, D. and Elia, G. (2004). Purification of biotinylated proteins on streptavidin resin: a protocol for quantitative elution. Proteomics 4, 2296-2299.

Ryšlavá, H., Doubnerová, V., Kavan, D. and Vaněk, O. (2013). Effect of posttranslational modifications on enzyme function and assembly. J. Proteomics 92, 80-109.

Saada, A., Vogel, R.O., Hoefs, S.J., van den Brand, M.A., Wessels, H.J., Willems, P.H., Venselaar, H., Shaag, A., Barghuti, F., Reish, O., Shohat, M., Huynen, M.A., Smeitink, J.A., van den Heuvel, L.P. and Nijtmans, L.G. (2009). Mutations in NDUFAF3 (C3ORF60), encoding an NDUFAF4 (C6ORF66)-interacting complex I assembly protein, cause fatal neonatal mitochondrial disease. Am. J. Hum. Genet. 84, 718-727.

Saddar, S., Dienhart, M.K., Stuart, R.A. (2008). The $F_{1} F_{0}-A T P$ synthase complex influences the assembly state of the cytochrome $b c_{1}$-cytochrome oxidase supercomplex and its association with the TIM23 machinery. J. Biol. Chem. 283, 6677-6686.

Sakakura, M. and Takayama, M. (2010). In-source decay and fragmentation characteristics of peptides using 5-aminosalicylic acid as a matrix in matrix-assisted laser desorption/ionization mass spectrometry. J. Am. Soc. Mass Spectrom. 21, 979-988.

Sala, A., Bordes, P. and Genevaux, P. (2014). Multitasking SecB chaperones in bacteria. Front. Microbiol. 5:666.

Santoni, V., Molloy, M. and Rabilloud, T. (2000). Membrane proteins and proteomics: un amour impossible? Electrophoresis 21, 1054-1070.

Sapra, R. (2009). The use of difference in-gel electrophoresis for quantitation of protein expression. Methods Mol. Biol. 492, 93-112.

Saraogi, I. and Shan, S.O. (2014). Co-translational protein targeting to the bacterial membrane. Biochim. Biophys. Acta 1843, 1433-1441.

Sargent, F. (2007). Constructing the wonders of the bacterial world: biosynthesis of complex enzymes. Microbiology 153, 633-651.

Savas, J.N., Stein, B.D., Wu, C.C. and Yates, J.R. 3rd. (2011). Mass spectrometry accelerates membrane protein analysis. Trends Biochem. Sci. 36, 388-396.

Schäfer, E., Dencher, N.A., Vonck, J. and Parcej, D.N. (2007). Three-dimensional structure of the respiratory chain supercomplex I1III2IV1 from bovine heart mitochondria. Biochemistry 46, 12579-12585.

Schägger, H. (2002). Respiratory chain supercomplexes of mitochondria and bacteria. Biochim. Biophys. Acta 1555, 154-159.

Schägger, H. and Pfeiffer, K. (2000). Supercomplexes in the respiratory chains of yeast and mammalian mitochondria. EMBO J. 19, 1777-1783.

Schägger, H., Aquila, H., Von Jagow, G. (1988). Coomassie blue-sodium dodecyl sulfatepolyacrylamide gel electrophoresis for direct visualization of polypeptides during electrophoresis. Anal. Biochem. 173, 201-205. 
Schägger, H. and von Jagow, G. (1991). Blue native electrophoresis for isolation of membrane protein complexes in enzymatically active form. Anal. Biochem. 199, 223-231.

Scheurer, S.B., Rybak, J.N., Roesli, C., Brunisholz, R.A., Potthast, F., Schlapbach, R., Neri, D. and Elia, G. (2005). Identification and relative quantification of membrane proteins by surface biotinylation and two-dimensional peptide mapping. Proteomics 5, 2718-2728.

Schiapparelli, L.M., McClatchy, D.B., Liu, H.H., Sharma, P., Yates. J.R. 3rd. and Cline, H.T. (2014). Direct detection of biotinylated proteins by mass spectrometry. J. Proteome Res. 13, 3966-3978.

Schluesener, D., Fischer, F., Kruip, J., Rögner, M. and Poetsch, A. (2005). Mapping the membrane proteome of Corynebacterium glutamicum. Proteomics 5, 1317-1330.

Schmidl, S.R., Gronau, K., Pietack, N., Hecker, M., Becher, D. and Stülke, J. (2010). The phosphoproteome of the minimal bacterium Mycoplasma pneumoniae: analysis of the complete known Ser/Thr kinome suggests the existence of novel kinases. Mol. Cell. Proteomics. 9, 12281242.

Schmidt, A., Müller, N., Schink, B. and Schleheck, D. (2013). A proteomic view at the biochemistry of syntrophic butyrate oxidation in Syntrophomonas wolfei. PLoS One 8:e56905.

Schmidt, C., Zhou, M., Marriott, H., Morgner, N., Politis, A. and Robinson, C.V. (2013). Comparative cross-linking and mass spectrometry of an intact F-type ATPase suggest a role for phosphorylation. Nat. Commun. 4:1985.

Schmitt, E., Naveau, M. and Mechulam, Y. (2010). Eukaryotic and archaeal translation initiation factor 2: a heterotrimeric tRNA carrier. FEBS Lett. 584, 405-412.

Schroeter, R., Voigt, B., Jürgen, B., Methling, K., Pöther, D.C., Schäfer, H., Albrecht, D., Mostertz, J., Mäder, U., Evers, S., Maurer, K.H., Lalk, M., Mascher, T., Hecker, M. and Schweder, T. (2011). The peroxide stress response of Bacillus licheniformis. Proteomics 11, 2851-2866.

Schubert, H.L., Blumenthal, R.M. and Cheng, X. (2003). Many paths to methyltransfer: a chronicle of convergence. Trends Biochem. Sci. 28, 329-335.

Seidler, J., Zinn, N., Boehm, M. E. and Lehmann, W.D. (2010). De novo sequencing of peptides by MS/MS. Proteomics 10, 634-649.

Sestak, J., Moravcova, D. and Kahle, V. (2015). Instrument platforms for nano liquid chromatography. J. Chromatogr. A 1421, 2-17.

Sevcenco, A.M., Hagen, W.R. and Hagedoorn, P.L. (2012). Microbial metalloproteomes explored using MIRAGE. Chem. Biodivers. 9, 1967-1980.

Shan, Y., Lai, Y. and Yan, A. (2012). Metabolic reprogramming under microaerobic and anaerobic conditions in bacteria. Subcell. Biochem. 64, 159-179.

Shi, L., Pigeonneau, N., Ravikumar, V., Dobrinic, P., Macek, B., Franjevic, D., Noirot-Gros, M.F. and Mijakovic, I. (2014). Cross-phosphorylation of bacterial serine/threonine and tyrosine protein kinases on key regulatory residues. Front. Microbiol. 5:495.

Shi, L. and Tu, B.P. (2015). Acetyl-CoA and the regulation of metabolism: mechanisms and consequences. Curr. Opin. Cell Biol. 33, 125-131.

Shi, W. and Chance, M.R. (2011). Metalloproteomics: forward and reverse approaches in metalloprotein structural and functional characterization. Curr. Opin. Chem. Biol. 15, 144-148.

Shoubridge, E.A. (2012). Supersizing the mitochondrial respiratory chain. Cell. Metab. 15, 271-272.

Sieber, J.R., Crable, B.R., Sheik, C.S., Hurst, G.B., Rohlin, L., Gunsalus, R.P. and McInerney, M.J. (2015). Proteomic analysis reveals metabolic and regulatory systems involved in the syntrophic and axenic lifestyle of Syntrophomonas wolfei. Front. Microbiol. 6:115.

Simon, J. and Kern, M. (2008). Quinone-reactive proteins devoid of haem b form widespread membrane-bound electron transport modules in bacterial respiration. Biochem. Soc. Trans. 36, 1011-1016.

Sinha, S., Kosalai, K., Arora, S., Namane, A., Sharma, P., Gaikwad, A.N., Brodin P. and Cole, S.T. (2005). Immunogenic membrane-associated proteins of Mycobacterium tuberculosis revealed by proteomics. Microbiology 151, 2411-2419.

Sinz, A., Arlt, C., Chorev, D. and Sharon, M. (2015). Chemical cross-linking and native mass spectrometry: A fruitful combination for structural biology. Protein Sci. 24, 1193-1209.

Smith, D.R., Doucette-Stamm, L.A., Deloughery, C., Lee, H., Dubois, J., Aldredge, T., Bashirzadeh, R., Blakely, D., Cook, R., Gilbert, K., Harrison, D., Hoang, L., Keagle, P., Lumm, W., Pothier, B., Qiu, D., Spadafora, R., Vicaire, R., Wang, Y., Wierzbowski, J., Gibson, R., Jiwani, N., Caruso, A., 
Bush, D., Reeve, J.N., et al. (1997). Complete genome sequence of Methanobacterium thermoautotrophicum deltaH: functional analysis and comparative genomics. J. Bacteriol. 179, 7135-7155.

Smith, L.M., Kelleher, N.L. and Consortium for Top Down, P. (2013). Proteoform: a single term describing protein complexity. Nat. Methods 10, 186-187.

Smith, P.M., Fox, J.L. and Winge, D.R. (2012). Biogenesis of the cytochrome $b c_{1}$ complex and role of assembly factors. Biochim. Biophys. Acta 1817, 276-286.

Snijders, A.P., Hung, M.L., Wilson, S.A. and Dickman, M.J. (2010). Analysis of arginine and lysine methylation utilizing peptide separations at neutral $\mathrm{pH}$ and electron transfer dissociation mass spectrometry. J. Am. Soc. Mass Spectrom. 21, 88-96.

Soares, N.C., Spät, P., Krug, K. and Macek, B. (2013). Global dynamics of the Escherichia coli proteome and phosphoproteome during growth in minimal medium. J. Proteome Res. 12, 26112621.

Sokolova, L., Wittig, I., Barth, H.D., Schägger, H., Brutschy, B. and Brandt, U. (2010). Laser-induced liquid bead ion desorption-MS of protein complexes from blue-native gels, a sensitive top-down proteomic approach. Proteomics 10, 1401-1407.

Soppa, J. (2010). Protein acetylation in archaea, bacteria, and eukaryotes. Archaea. 2010. pii: 820681.

Soufi, B and Macek, B. (2015). Global analysis of bacterial membrane proteins and their modifications. Int. J. Med. Microbiol. 305, 203-208.

Soufi, B., Gnad, F., Jensen, P.R., Petranovic, D., Mann, M., Mijakovic, I. and Macek, B. (2008). The Ser/Thr/Tyr phosphoproteome of Lactococcus lactis IL1403 reveals multiply phosphorylated proteins. Proteomics 8, 3486-3493.

Soufi, B., Soares, N.C., Ravikumar, V. and Macek, B. (2012). Proteomics reveals evidence of cross-talk between protein modifications in bacteria: focus on acetylation and phosphorylation. Curr. Opin. Microbiol. 15, 357-363.

Soufi, B., Krug, K., Harst, A. and Macek, B. (2015). Characterization of the E. coli proteome and its modifications during growth and ethanol stress. Front. Microbiol. 6:103.

Soung, G.Y., Miller, J.L., Koc, H. and Koc E.C. (2009). Comprehensive analysis of phosphorylated proteins of Escherichia coli ribosomes. J. Proteome Res. 8, 3390-3402.

Sousa, P.M., Silva, S.T., Hood, B.L., Charro, N., Carita, J.N., Vaz, F., Penque, D., Conrads, T.P. and Melo, A.M. (2011). Supramolecular organizations in the aerobic respiratory chain of Escherichia coli. Biochimie 93, 418-425.

Sousa, P.M., Videira, M.A., Bohn, A., Hood, B.L., Conrads, T.P., Goulao, L.F. and Melo, A.M. (2012). The aerobic respiratory chain of Escherichia coli: from genes to supercomplexes. Microbiology 158, 2408-2418.

Sousa, P.M., Videira, M.A. and Melo, A.M. (2013a). The formate:oxygen oxidoreductase supercomplex of Escherichia coli aerobic respiratory chain. FEBS Lett. 587, 2559-2564.

Sousa, P.M., Videira, M.A., Santos, F.A., Hood, B.L., Conrads, T.P. and Melo AM (2013b). The bc:caa 3 supercomplexes from the Gram positive bacterium Bacillus subtilis respiratory chain: a megacomplex organization? Arch. Biochem. Biophys. 537, 153-160.

Spät, P., Maček, B. and Forchhammer, K. (2015). Phosphoproteome of the cyanobacterium Synechocystis sp. PCC 6803 and its dynamics during nitrogen starvation. Front. Microbiol. 6:248.

Spiro S. (2012). Nitrous oxide production and consumption: regulation of gene expression by gassensitive transcription factors. Philos. Trans. R. Soc. Lond. B Biol. Sci. 367, 1213-1225.

Srinivasa, S., Ding, X. and Kast, J. (2015). Formaldehyde cross-linking and structural proteomics: Bridging the gap. Methods 89, 91-98.

Stangl, M. and Schneider, D. (2015). Functional competition within a membrane: Lipid recognition vs. transmembrane helix oligomerization. Biochim. Biophys. Acta 1848, 1886-1896.

Steen, H. and Mann, M. (2004). The ABC's (and XYZ's) of peptide sequencing. Nat. Rev. Mol. Cell Biol. 5, 699-711.

Stemmann, O., Zou, H., Gerber, S. A., Gygi, S.P. and Kirschner, M.W. (2001). Dual inhibition of sister chromatid separation at metaphase. Cell 107, 715-726.

Stojko, J., Fieulaine, S., Petiot-Bécard, S., Van Dorsselaer, A., Meinnel, T., Giglione, C. and Cianférani, S. (2015). Ion mobility coupled to native mass spectrometry as a relevant tool to investigate extremely small ligand-induced conformational changes. Analyst 140, 7234-7245. 
Strader, M.B., Verberkmoes, N.C., Tabb, D.L., Connelly, H.M., Barton, J.W., Bruce, B.D., Pelletier, D.A., Davison, B.H., Hettich, R.L., Larimer, F.W. and Hurst, G.B. (2004). Characterization of the 70S Ribosome from Rhodopseudomonas palustris using an integrated "top-down" and "bottomup" mass spectrometric approach. J. Proteome Res. 3, 965-978.

Strecker, V., Wumaier, Z., Wittig, I. and Schägger, H. (2010). Large pore gels to separate mega protein complexes larger than $10 \mathrm{MDa}$ by blue native electrophoresis: isolation of putative respiratory strings or patches. Proteomics 10, 3379-3387.

Stroh, A., Anderka, O., Pfeiffer, K., Yagi, T., Finel, M., Ludwig, B. and Schägger, H. (2004). Assembly of respiratory complexes I, III, and IV into NADH oxidase supercomplex stabilizes complex I in Paracoccus denitrificans. J. Biol. Chem. 279, 5000-5007.

Stülke, J. and Hillen, W. (1998). Coupling physiology and gene regulation in bacteria: the phosphotransferase sugar uptake system delivers the signals. Naturwissenschaften 85, 583-592.

Suenaga, H. (2015). Targeted metagenomics unveils the molecular basis for adaptive evolution of enzymes to their environment. Front. Microbiol. 6:1018.

Suh, M.J., Kuntumalla, S., Yu, Y. and Pieper, R. (2014). Proteomes of pathogenic Escherichia coli/Shigella group surveyed in their host environments. Expert Rev. Proteomics. 11, 593-609.

Sun, X., Chiu, J.F., He, Q.Y. (2008). Fractionation of proteins by immobilized metal affinity chromatography. Methods Mol. Biol. 424, 205-212.

Sun, X., Ge, F., Xiao, C.L., Yin, X.F., Ge, R., Zhang, L.H. and He, Q.Y. (2010). Phosphoproteomic analysis reveals the multiple roles of phosphorylation in pathogenic bacterium Streptococcus pneumoniae. J. Proteome Res. 9, 275-282.

Sun, X., Jia, H.L., Xiao, C.L., Yin, X.F., Yang, X.Y., Lu, J., He, X., Li, N., Li, H. and He Q.Y. (2011). Bacterial proteome of Streptococcus pneumoniae through multidimensional separations coupled with LC-MS/MS. OMICS 15, 477-482.

Syka, J.E., Marto, J.A., Bai, D.L., Horning, S., Senko, M.W., Schwartz, J.C., Ueberheide, B., Garcia, B., Busby, S., Muratore, T., Shabanowitz, J. and Hunt, D.F. (2004). Novel linear quadrupole ion trap/FT mass spectrometer: performance characterization and use in the comparative analysis of histone H3 post-translational modifications. J. Proteome Res. 3, 621-626.

Tahara, M., Ohsawa, A., Saito, S. and Kimura, M. (2004). In vitro phosphorylation of initiation factor 2 alpha (aIF2 alpha) from hyperthermophilic archaeon Pyrococcus horikoshii OT3. J. Biochem. 135, 479-485.

Tainer, J.A. and Adams, M.W. (2010). Microbial metalloproteomes are largely uncharacterized. Nature 466, 779-782.

Tang, S., Guo, Y., Xiong, C., Liu, S., Liu, X. and Jiang, S. (2014). Nanoparticle-based monoliths for chromatographic separations. Analyst 139, 4103-4117.

Taylor, J.A. and Johnson, R.S. (1997). Sequence database searches via de novo peptide sequencing by tandem mass spectrometry. Rapid Commun. Mass Spectrom. 11, 1067-1075.

Temperton, B. and Giovannoni, S.J. (2012). Metagenomics: microbial diversity through a scratched lens. Curr. Opin. Microbiol. 15, 605-612.

Teramoto, H., Inui, M. and Yukawa, H. (2013). OxyR acts as a transcriptional repressor of hydrogen peroxide-inducible antioxidant genes in Corynebacterium glutamicum R. FEBS J. 280, 3298-3312.

Terpe, K. (2003). Overview of tag protein fusions: from molecular and biochemical fundamentals to commercial systems. Appl. Microbiol. Biotechnol. 60, 523-533.

Thauer, R.K., Kaster, A.K., Seedorf, H., Buckel, W. and Hedderich, R. (2008). Methanogenic archaea: ecologically relevant differences in energy conservation. Nat. Rev. Microbiol. 6, 579-591.

Thompson, A., Schafer, J., Kuhn, K., Kienle, S., Schwarz, J., Schmidt, G., Neumann, T., Johnstone, R., Mohammed, A.K. and Hamon, C. (2003). Tandem mass tags: a novel quantification strategy for comparative analysis of complex protein mixtures by MS/MS. Anal. Chem. 75, 1895-1904.

Tipton, J.D., Tran, J.C., Catherman, A.D., Ahlf, D.R., Durbin, K.R. and Kelleher, N.L. (2011). Analysis of intact protein isoforms by mass spectrometry. J. Biol. Chem. 286, 25451-25458.

Tosha, T. and Shiro, Y. (2013). Crystal structures of nitric oxide reductases provide key insights into functional conversion of respiratory enzymes. IUBMB Life 65, 2217-26.

Tran, B.Q., Goodlett, D.R. and Goo, Y.A. (2015). Advances in protein complex analysis by chemical cross-linking coupled with mass spectrometry (CXMS) and bioinformatics. Biochim. Biophys. Acta 1864, 123-129. 
Trinkle-Mulcahy, L. (2012). Resolving protein interactions and complexes by affinity purification followed by label-based quantitative mass spectrometry. Proteomics 12, 1623-1638.

Tripodi, F., Nicastro, R., Reghellin, V. and Coccetti, P. (2015). Post-translational modifications on yeast carbon metabolism: Regulatory mechanisms beyond transcriptional control. Biochim. Biophys. Acta 1850, 620-627.

Trouillard, M., Meunier, B. and Rappaport, F. (2011). Questioning the functional relevance of mitochondrial supercomplexes by time-resolved analysis of the respiratory chain. Proc. Natl. Acad. Sci. U S A 108, E1027-1034.

Tsou, C.C., Tsai, C.F., Tsui, Y.H., Sudhir, P.R., Wang, Y.T., Chen, Y.J., Chen, J.Y., Sung, T.Y. and Hsu, W.L. (2010). IDEAL-Q, an automated tool for label-free quantitation analysis using an efficient peptide alignment approach and spectral data validation. Mol. Cell. Proteomics 9, 131144.

Tsutsui, Y. and Wintrode, P.L. (2007). Hydrogen/deuterium exchange-mass spectrometry: a powerful tool for probing protein structure, dynamics and interactions. Curr. Med. Chem. 14, 2344-2358.

Tucker, E.J., Wanschers, B.F., Szklarczyk, R., Mountford, H.S., Wijeyeratne, X.W., van den Brand, M.A., Leenders, A.M., Rodenburg, R.J., Reljić, B., Compton, A.G., Frazier, A.E., Bruno, D.L., Christodoulou, J., Endo, H., Ryan, M,T., Nijtmans, L.G., Huynen, M.A. and Thorburn, D.R. (2013). Mutations in the UQCC1-interacting protein, UQCC2, cause human complex III deficiency associated with perturbed cytochrome $b$ protein expression. PLoS Genet. 9:e1004034.

Valsecchi, F., Ramos-Espiritu, L.S., Buck, J., Levin, L.R. and Manfredi, G. (2013). cAMP and mitochondria. Physiology (Bethesda) 28, 199-209.

Valsecchi, F., Konrad, C. and Manfredi, G. (2014). Role of soluble adenylyl cyclase in mitochondria. Biochim. Biophys. Acta 1842, 2555-2560.

van den Ecker, D., van den Brand, M.A., Bossinger, O., Mayatepek, E., Nijtmans, L.G. and Distelmaier, F. (2010). Blue native electrophoresis to study mitochondrial complex I in C. elegans. Anal. Biochem. 407, 287-289.

van Gestel, R.A., Rijken, P.J., Surinova, S., O'Flaherty, M., Heck, A.J., Killian, J.A., de Kroon, A.I. and Slijper, M. (2010). The influence of the acyl chain composition of cardiolipin on the stability of mitochondrial complexes; an unexpected effect of cardiolipin in alpha-ketoglutarate dehydrogenase and prohibitin complexes. J. Proteomics 73, 806-814.

van Noort, V., Seebacher, J., Bader, S., Mohammed, S., Vonkova, I., Betts, M.J., Kühner, S., Kumar, R., Maier, T., O'Flaherty, M., Rybin, V., Schmeisky, A., Yus, E., Stülke, J., Serrano, L., Russell, R.B., Heck, A.J., Bork, P. and Gavin, A.C. (2012). Cross-talk between phosphorylation and lysine acetylation in a genome-reduced bacterium. Mol. Syst. Biol. 8:571.

van Schaik, W. (2015). The human gut resistome. Philos. Trans. R. Soc. Lond. B. Biol. Sci. 370, 20140087. doi: 10.1098/rstb.2014.0087.

VanBogelen, R.A., Olson, E.R., Wanner, B.L. and Neidhardt, F.C. (1996). Global analysis of proteins synthesized during phosphorus restriction in Escherichia coli. J. Bacteriol. 178, 4344-4366.

Vartak, R., Porras, C.A. an Bai, Y. (2013). Respiratory supercomplexes: structure, function and assembly. Protein Cell 4, 582-590.

Vartak, R.S., Semwal, M.K. and Bai, Y. (2014). An update on complex I assembly: the assembly of players. J. Bioenerg. Biomembr. 46, 323-328.

Vassilopoulos, A., Pennington, J.D., Andresson, T., Rees, D.M., Bosley, A.D., Fearnley, I.M., Ham, A., Flynn, C.R., Hill, S., Rose, K.L., Kim, H.S., Deng, C.X., Walker, J.E. and Gius, D. (2014). SIRT3 deacetylates ATP synthase F1 complex proteins in response to nutrient- and exercise-induced stress. Antioxid. Redox Signal. 21, 551-564.

Venable, J.D., Steckler, C., Ou, W., Grünewald, J., Agarwalla S. and Brock, A. Isotope-Coded Labeling for Accelerated Protein Interaction Profiling Using MS. Anal. Chem. 87, 7540-7504.

Venne, A.S., Kollipara, L. and Zahedi, R.P. (2014). The next level of complexity: crosstalk of posttranslational modifications. Proteomics 14, 513-524.

Verissimo, A.F. and Daldal, F. (2014). Cytochrome $c$ biogenesis System I: an intricate process catalyzed by a maturase supercomplex? Biochim. Biophys. Acta 1837, 989-998.

Vermeulen, M., Hubner, N.C. and Mann, M. (2008). High confidence determination of specific proteinprotein interactions using quantitative mass spectrometry. Curr. Opin. Biotechnol. 19, 331-337. 
Villén, J. and Gygi, S.P. (2008). The SCX/IMAC enrichment approach for global phosphorylation analysis by mass spectrometry. Nat. Protoc. 3, 1630-1638.

Viswanathan, S., Unlü, M. and Minden, J.S. (2006). Two-dimensional difference gel electrophoresis. Nat. Protoc. 1, 1351-1358.

Vogel, R.O., Janssen, R.J., Ugalde, C., Grovenstein, M., Huijbens, R.J., Visch, H.J., van den Heuvel, L.P., Willems, P.H., Zeviani, M., Smeitink, J.A. and Nijtmans, L.G. (2005). Human mitochondrial complex I assembly is mediated by NDUFAF1. FEBS J. 272, 5317-5326.

Vogel, R.O., Dieteren, C.E., van den Heuvel, L.P., Willems, P.H., Smeitink, J.A., Koopman, W.J. and Nijtmans, L.G. (2007a). Identification of mitochondrial complex I assembly intermediates by tracing tagged NDUFS3 demonstrates the entry point of mitochondrial subunits. J. Biol. Chem. 282, 7582-7590.

Vogel, R.O., Janssen, R.J., van den Brand, M.A., Dieteren, C.E., Verkaart, S., Koopman, W.J., Willems, P.H., Pluk, W., van den Heuvel, L.P., Smeitink, J.A. and Nijtmans, L.G. (2007b). Cytosolic signaling protein Ecsit also localizes to mitochondria where it interacts with chaperone NDUFAF1 and functions in complex I assembly. Genes Dev. 21, 615-624.

Vogel, R.O., Smeitink, J.A. and Nijtmans, L.G. (2007c). Human mitochondrial complex I assembly: a dynamic and versatile process. Biochim. Biophys. Acta 1767, 1215-1227.

Voisin, S., Watson, D.C., Tessier, L., Ding, W., Foote, S., Bhatia, S., Kelly, J.F. and Young, N.M. (2007). The cytoplasmic phosphoproteome of the Gram-negative bacterium Campylobacter jejuni: evidence for modification by unidentified protein kinases. Proteomics 7, 4338-4348.

Völkel, P., Le Faou, P. and Angrand, P.O. (2010). Interaction proteomics: characterization of protein complexes using tandem affinity purification-mass spectrometry. Biochem. Soc. Trans. 38, 883887.

Völker, U. and Hecker, M. (2005). From genomics via proteomics to cellular physiology of the Grampositive model organism Bacillus subtilis. Cell. Microbiol. 7, 1077-1085.

Vonck, J. and Schäfer, E. (2009). Supramolecular organization of protein complexes in the mitochondrial inner membrane. Biochim. Biophys. Acta 1793, 117-124.

Vuckovic, D., Dagley, L.F., Purcell, A.W. and Emili, A. (2013). Membrane proteomics by high performance liquid chromatography-tandem mass spectrometry: Analytical approaches and challenges. Proteomics 13, 404-423.

Wagner, G.R. and Hirschey, M.D. (2014). Nonenzymatic protein acylation as a carbon stress regulated by sirtuin deacylases. Mol. Cell. 54, 5-16.

Walpole, T.B., Palmer, D.N., Jiang, H., Ding, S., Fearnley, I.M. and Walker, J.E. (2015). Conservation of complete trimethylation of lysine-43 in the rotor ring of c-subunits of metazoan adenosine triphosphate (ATP) synthases. Mol. Cell. Proteomics 14, 828-840.

Wang, B., Xu, M. and Sun, G. (2010). Comparative analysis of membranous proteomics of Shewanella decolorationis S12 grown with azo compound or Fe (III) citrate as sole terminal electron acceptor. Appl. Microbiol. Biotechnol. 86, 1513-1523.

Wang, H., Straubinger, R.M., Aletta, J.M., Cao, J., Duan, X., Yu, H. and Qu, J. (2009). Accurate localization and relative quantification of arginine methylation using nanoflow liquid chromatography coupled to electron transfer dissociation and orbitrap mass spectrometry. J. Am. Soc. Mass Spectrom. 20, 507-519.

Wang, L., Pan, L. and Tao, W.A. (2014). Specific visualization and identification of phosphoproteome in gels. Anal Chem. 86, 6741-6747.

Wang, Q., Zhang, Y., Yang, C., Xiong, H., Lin, Y., Yao, J., Li, H., Xie, L., Zhao, W., Yao, Y., Ning, Z.B., Zeng, R., Xiong, Y., Guan, K.L., Zhao, S. and Zhao, G.P. (2010). Acetylation of metabolic enzymes coordinates carbon source utilization and metabolic flux. Science 327, 1004-1007.

Wang, Y.K., Ma, Z., Quinn, D.F. and Fu, E.W. (2002). Inverse 15N-metabolic labeling/mass spectrometry for comparative proteomics and rapid identification of protein markers/targets. Rapid Commun. Mass Spectrom. 16, 1389-1397.

Washburn, M.P, Wolters, D. and Yates JR 3rd. (2001). Large-scale analysis of the yeast proteome by multidimensional protein identification technology. Nat. Biotechnol. 19, 242-247.

Washburn, M.P. (2004). Utilisation of proteomics datasets generated via multidimensional protein identification technology (MudPIT). Brief. Funct. Genomic Proteomic. 3, 280-286. 
Watanabe, M., Kubota, H., Wada, H., Narikawa, R. and Ikeuchi, M. (2011). Novel supercomplex organization of photosystem I in Anabaena and Cyanophora paradoxa. Plant Cell. Physiol. 52, 162-168.

Watanabe, M., Semchonok, D.A., Webber-Birungi, M.T., Ehira, S., Kondo, K., Narikawa, R., Ohmori, M., Boekema, E.J. and Ikeuchi, M. (2014). Attachment of phycobilisomes in an antennaphotosystem I supercomplex of cyanobacteria. Proc. Natl. Acad. Sci. U S A 111, 2512-2517.

Wayne Outten, F. (2015). Recent advances in the Suf Fe-S cluster biogenesis pathway: Beyond the Proteobacteria. Biochim. Biophys. Acta 1853, 1464-1469.

Weatherly, D.B., Atwood, J.A., 3rd, Minning, T.A., Cavola, C., Tarleton, R.L. and Orlando, R. (2005). A Heuristic method for assigning a false-discovery rate for protein identifications from Mascot database search results. Mol. Cell. Proteomics 4, 762-772.

Weber, T.A., Koob, S., Heide, H., Wittig, I., Head, B., van der Bliek, A., Brandt, U., Mittelbronn, M. and Reichert, A.S. (2013). APOOL is a cardiolipin-binding constituent of the Mitofilin/MINOS protein complex determining cristae morphology in mammalian mitochondria. PLoS One 8:e63683.

Wegener, K.M., Singh, A.K., Jacobs, J.M., Elvitigala, T., Welsh, E.A., Keren, N., Gritsenko, M.A., Ghosh, B.K., Camp, D.G. 2nd., Smith, R.D. and Pakrasi, H.B. (2010). Global proteomics reveal an atypical strategy for carbon/nitrogen assimilation by a cyanobacterium under diverse environmental perturbations. Mol. Cell. Proteomics. 9, 2678-2689.

Wei, C., Yang, J., Zhu, J., Zhang, X., Leng, W., Wang, J., Xue, Y., Sun, L., Li, W., Wang, J. and Jin, Q. (2006). Comprehensive proteomic analysis of Shigella flexneri 2a membrane proteins. $J$. Proteome Res. 5, 1860-1865.

Wei, J., Sun, J., Yu, W., Jones, A., Oeller, P., Keller, M., Woodnutt, G. and Short, J.M. (2005). Global proteome discovery using an online three-dimensional LC-MS/MS. J. Proteome Res. 4, 801-808.

Weiner, J.H. and Li, L. (2008). Proteome of the Escherichia coli envelope and technological challenges in membrane proteome analysis. Biochim. Biophys. Acta 1778, 1698-1713.

Weinert, B.T., Schölz, C. Wagner, S.A, Iesmantavicius, V., Su, D. Daniel, J.A. and Choudhary, C. (2013). Lysine succinylation is a frequently occurring modification in prokaryotes and eukaryotes and extensively overlaps with acetylation. Cell Rep. 4, 842-851.

Wenz, T., Hielscher, R., Hellwig, P., Schägger, H., Richers, S. and Hunte, C. (2009). Role of phospholipids in respiratory cytochrome $b c_{1}$ complex catalysis and supercomplex formation. Biochim. Biophys. Acta 1787, 609-616.

Wepf, A., Glatter, T., Schmidt, A., Aebersold, R. and Gstaiger, M. (2009). Quantitative interaction proteomics using mass spectrometry. Nat. Methods 6, 203-205.

Werner, T., Sweetman, G., Savitski, M.F., Mathieson, T., Bantscheff, M. and Savitski, M.M. (2014). Ion coalescence of neutron encoded TMT 10-plex reporter ions. Anal. Chem. 86, 3594-3601.

Wessels, H.J.. (2015). Mitochondrial proteomics: method development and application. PhD thesis. Radboud University Nijmegen.

Wessels, H.J., Vogel, R.O., van den Heuvel, L., Smeitink, J.A., Rodenburg, R.J., Nijtmans, L.G. and Farhoud, M.H. (2009). LC-MS/MS as an alternative for SDS-PAGE in blue native analysis of protein complexes. Proteomics 9, 4221-4228.

Wessels, H.J., Vogel, R.O., Lightowlers, R.N., Spelbrink, J.N., Rodenburg, R.J., van den Heuvel, L.P., van Gool, A.J., Gloerich, J., Smeitink, J.A. and Nijtmans, L.G. (2013). Analysis of 953 human proteins from a mitochondrial HEK293 fraction by complexome profiling. PLoS One 8:e68340.

Whitelegge, J.P. (2002). Plant proteomics: BLASTing out of a MudPIT. Proc. Natl. Acad. Sci. U S A 99, 11564-11566.

Wickström, D., Wagner, S., Baars, L., Ytterberg, A.J., Klepsch, M., van Wijk, K.J., Luirink, J. and de Gier J.W. (2011a). Consequences of depletion of the signal recognition particle in Escherichia coli. J. Biol. Chem. 286, 4598-4609.

Wickström, D., Wagner, S., Simonsson, P., Pop, O., Baars, L., Ytterberg, A.J., van Wijk, K.J., Luirink, J. and de Gier, J.W. (2011b). Characterization of the consequences of YidC depletion on the inner membrane proteome of E. coli using 2D blue native/SDS-PAGE. J. Mol. Biol. 409, 124-135.

Wilmes, P. and Bond, P.L. (2004). The application of two-dimensional polyacrylamide gel electrophoresis and downstream analyses to a mixed community of prokaryotic microorganisms. Environ. Microbiol. 6, 911-920. 
Wilson, M.M., Anderson, D.E. and Bernstein, H.D. (2015). Analysis of the outer membrane proteome and secretome of Bacteroides fragilis reveals a multiplicity of secretion mechanisms. PLoS One 10: 0117732 .

Wittig, I. and Schägger, H. (2005). Advantages and limitations of clear-native PAGE. Proteomics 5, 4338-4346.

Wittig, I. and Schägger, H. (2008a). Features and applications of blue-native and clear-native electrophoresis. Proteomics 8, 3974-3990.

Wittig, I. and Schägger, H. (2008b). Structural organization of mitochondrial ATP synthase. Biochim. Biophys. Acta 1777, 592-598.

Wittig, I., Schägger, H. (2009a). Native electrophoretic techniques to identify protein-protein interactions. Proteomics 9, 5214-5223.

Wittig, I. and Schägger, H. (2009b). Supramolecular organization of ATP synthase and respiratory chain in mitochondrial membranes. Biochim. Biophys. Acta 1787, 672-680.

Wittig, I., Braun, H.P. and Schägger, H. (2006a). Blue native PAGE. Nat. Protoc. 1, 418-428.

Wittig, I., Carrozzo, R., Santorelli, F.M. and Schägger, H. (2006b). Supercomplexes and subcomplexes of mitochondrial oxidative phosphorylation. Biochim. Biophys. Acta 1757, 1066-1072.

Wittig, I., Karas, M. and Schägger, H. (2007). High resolution clear native electrophoresis for in-gel functional assays and fluorescence studies of membrane protein complexes. Mol. Cell. Proteomics. 6, $1215-1225$.

Wittig, I., Velours, J., Stuart, R. and Schägger, H. (2008). Characterization of domain interfaces in monomeric and dimeric ATP synthase. Mol. Cell. Proteomics 7, 995-1004.

Wittig, I., Beckhaus, T., Wumaier, Z., Karas, M. and Schägger, H. (2010). Mass estimation of native proteins by blue native electrophoresis: principles and practical hints. Mol. Cell. Proteomics. 9, 2149-2161.

Wolters, D.A., Washburn, M.P. and Yates J.R. 3rd. (2001). An automated multidimensional protein identification technology for shotgun proteomics. Anal. Chem. 73, 5683-5690.

Wright, J.C., Collins, M.O., Yu, L., Kall, L., Brosch, M. and Choudhary, J.S. (2012). Enhanced peptide identification by electron transfer dissociation using an improved Mascot Percolator. Mol. Cell. Proteomics 11, 478-491.

Wu, C.C., MacCoss, M.J., Howell, K.E. and Yates, J.R. 3rd. (2003). A method for the comprehensive proteomic analysis of membrane proteins. Nat. Biotechnol. 21, 532-538.

Wu, S., Lourette, N.M., Tolić, N., Zhao, R., Robinson, E.W., Tolmachev, A.V., Smith, R.D. and PasaTolić, L. (2009). An integrated top-down and bottom-up strategy for broadly characterizing protein isoforms and modifications. J. Proteome Res. 8, 1347-1357.

Wu, X., Vellaichamy, A., Wang, D., Zamdborg, L., Kelleher, N.L., Huber, S.C. and Zhao, Y. (2013). Differential lysine acetylation profiles of Erwinia amylovora strains revealed by proteomics. $J$. Proteomics 79, 60-71.

Wu, Y.T., Lee, H.C., Liao, C.C. and Wei, Y.H. (2013). Regulation of mitochondrial $F_{0} F_{1} A T P a s e$ activity by Sirt3-catalyzed deacetylation and its deficiency in human cells harboring 4977bp deletion of mitochondrial DNA. Biochim. Biophys. Acta 1832, 216-227.

Xie, L., Wang, X., Zeng, J., Zhou, M., Duan, X., Li, Q., Zhang, Z., Luo, H., Pang, L., Li, W., Liao, G., Yu, X., Li, Y., Huang, H. and Xie, J. (2015). Proteome-wide lysine acetylation profiling of the human pathogen Mycobacterium tuberculosis. Int. J. Biochem. Cell. Biol. 59, 193-202.

$\mathrm{Xu}$, H., Hegde, S.S. and Blanchard, J.S. (2011). Reversible acetylation and inactivation of Mycobacterium tuberculosis acetyl-CoA synthetase is dependent on cAMP. Biochemistry 50, 5883-5892.

Yamashita, M. and Fenn, J.B. (1984). Electrospray ion source. Another variation on the free-jet theme. J. Phys. Chem. 88, 4451-4459.

Yates, J.R. 3rd., Speicher, S., Griffin, P.R. and Hunkapiller, T. (1993). Peptide mass maps: a highly informative approach to protein identification. Anal. Biochem. 214, 397-408.

Yates, J.R., Ruse, C.I. and Nakorchevsky, A. (2009). Proteomics by mass spectrometry: approaches, advances, and applications. Annu. Rev. Biomed. Eng. 11, 49-79.

Yeagle, P.L. (2014). Non-covalent binding of membrane lipids to membrane proteins. Biochim. Biophys. Acta 1838, 1548-1559. 
Yokoyama, K. and Leimkühler, S. (2015). The role of FeS clusters for molybdenum cofactor biosynthesis and molybdoenzymes in bacteria. Biochim. Biophys. Acta 1853, 1335-1349.

Young, M.M., Tang, N., Hempel, J.C., Oshiro, C.M., Taylor, E.W., Kuntz, I.D., Gibson, B.W. and Dollinger, G. (2000). High throughput protein fold identification by using experimental constraints derived from intramolecular cross-links and mass spectrometry. Proc. Natl. Acad. Sci. U S A 97, 5802-5806.

Young, N.L., Plazas-Mayorca, M.D. and Garcia, B.A. (2010). Systems-wide proteomic characterization of combinatorial post-translational modification patterns. Expert Rev. Proteomics 7, 79-92.

Yu, B.J., Kim, J.A., Moon, J.H., Ryu, S.E. and Pan, J.G. (2008). The diversity of lysine-acetylated proteins in Escherichia coli. J. Microbiol. Biotechnol. 18, 1529-1536.

Zahedi, R.P., Meisinger, C. and Sickmann, A. (2005). Two-dimensional benzyldimethyl-nhexadecylammonium chloride/SDS-PAGE for membrane proteomics. Proteomics 5, 3581-3588.

Zahedi, R.P., Moebius, J., Sickmann, A. (2007). Two-dimensional BAC/SDS-PAGE for membrane proteomics. Subcell. Biochem. 43, 13-20.

Zamdborg, L., LeDuc, R.D., Glowacz, K.J., Kim, Y.B., Viswanathan, V., Spaulding, I. T., Early, B.P., Bluhm, E.J., Babai, S. and Kelleher, N.L. (2007). ProSight PTM 2.0: improved protein identification and characterization for top down mass spectrometry. Nucleic Acids Res. 35, W701706.

Zhang, J., Xin, L., Shan, B., Chen, W., Xie, M., Yuen, D., Zhang, W., Zhang, Z., Lajoie, G.A. and Ma, B. (2012). PEAKS DB: de novo sequencing assisted database search for sensitive and accurate peptide identification. Mol. Cell. Proteomics 11, M111010587.

Zhang, K., Zheng, S., Yang, J.S., Chen, Y. and Cheng, Z. (2013). Comprehensive profiling of protein lysine acetylation in Escherichia coli. J. Proteome Res. 12, 844-851.

Zhang, M., Mileykovskaya, E. and Dowhan, W. (2005). Cardiolipin is essential for organization of complexes III and IV into a supercomplex in intact yeast mitochondria. J. Biol. Chem. 280, 2940329408.

Zhang, M., Mileykovskaya, E. and Dowhan, W. (2002). Gluing the respiratory chain together. Cardiolipin is required for supercomplex formation in the inner mitochondrial membrane. J. Biol. Chem. 277, 43553-43556.

Zhang, Y., Fonslow, B.R., Shan, B., Baek, M. C. and Yates, J.R., 3rd. (2013). Protein analysis by shotgun/bottom-up proteomics. Chem. Rev. 113, 2343-2394.

Zhang, Y., Wu, Z.X., Wan, X.L., Liu, P., Zhang, J.B., Ye, Y., Zhao, Y.M. and Tan, M.J. (2014). Comprehensive profiling of lysine acetylome in Staphylococcus aureus. Sci. China Chem. 57, 732738.

Zhao, S., Xu, W., Jiang, W., Yu, W., Lin, Y., Zhang, T., Yao, J., Zhou, L., Zeng, Y., Li, H., Li, Y., Shi, J., An, W., Hancock, S.M., He, F., Qin, L., Chin, J., Yang, P., Chen, X., Lei, Q., Xiong, Y. and Guan, K.L. (2010). Regulation of cellular metabolism by protein lysine acetylation. Science 327, 1000-1004.

Zheng, J. and Jia, Z. (2010). Structure of the bifunctional isocitrate dehydrogenase kinase/ phosphatase. Nature 465, 961-965.

Zheng, J., Yates, S.P. and Jia, Z. (2012). Structural and mechanistic insights into the bifunctional enzyme isocitrate dehydrogenase kinase/phosphatase AceK. Philos. Trans. R. Soc. Lond. B Biol. Sci. 367, 2656-2668.

Zhou, M. and Robinson, C.V. (2010). When proteomics meets structural biology. Trends Biochem. Sci. 35, 522-529.

Zhou, M. and Robinson, C.V. (2014). Flexible membrane proteins: functional dynamics captured by mass spectrometry. Curr. Opin. Struct. Biol. 28, 122-130.

Zhou, M., Morgner, N., Barrera, N.P., Politis, A., Isaacson, S.C., Matak-Vinković, D., Murata, T., Bernal, R.A., Stock, D. and Robinson, C.V. (2011). Mass spectrometry of intact V-type ATPases reveals bound lipids and the effects of nucleotide binding. Science 334, 380-385.

Zhou, Y., Aebersold, R. and Zhang, H. (2007). Isolation of N-linked glycopeptides from plasma. Anal. Chem. 79, 5826-5837.

Zhou, Y., Dong, J. and Vachet, R.W. (2011). Electron transfer dissociation of modified peptides and proteins. Curr. Pharm. Biotechnol. 12, 1558-1567. 
Zijnge, V., Kieselbach, T. and Oscarsson, J. (2012). Proteomics of protein secretion by Aggregatibacter actinomycetemcomitans. PLoS One 7:e41662.

Zischka, H., Braun, R.J., Marantidis, E.P., Büringer, D., Bornhövd, C., Hauck, S.M., Demmer, O., Gloeckner, C.J., Reichert, A.S., Madeo, F. and Ueffing, M. (2006). Differential analysis of Saccharomyces cerevisiae mitochondria by free flow electrophoresis. Mol. Cell. Proteomics. 5, 2185-2200.

Zumft, W.G. and Kroneck, P.M. (2007). Respiratory transformation of nitrous oxide $\left(\mathrm{N}_{2} \mathrm{O}\right)$ to dinitrogen by Bacteria and Archaea. Adv. Microb. Physiol. 52, 107-227. 


\section{LEGENDS TO THE FIGURES}

Figure 1 ESI-MS spectra of the tryptic BSA peptide $\mathrm{YIC}_{(\mathrm{cam})}$ DNQDTISSK acquired using an ultra-high resolution quadrupole time-of-flight mass spectrometer (maXis 4G ETD, Bruker Daltonics). Mass differences between the isotopic peaks are annotated in atomic mass units (amu, u). (A) The isotopic pattern for the $z=2$ ion. An average mass difference of $0.50 \mathrm{u}$ for subsequent isotopic peaks is calculated from the spectrum, which can be used to determine the charge state of the ion: $z=1 / 0.5=2$. (B) The isotopic pattern for the $z=3$ ion. The average mass difference of $0.33 \mathrm{u}$ for subsequent isotopic peaks establishes $z=1 / 0.33=3$.

Figure 2 ESI-MS and deconvoluted MS spectra of horse cytochrome $c$. (A) The ESI-MS spectrum in which a range of different charge state peaks is observed for horse cytochrome $c$. (B) The deconvoluted neutral mass spectrum for cytochrome $c$. The mass measurement error for cytochrome $c$ was only 0.0102 Da or $0.826 \mathrm{ppm}$. Spectra were acquired using an ultra-high resolution quadrupole time-of-flight mass spectrometer (maxis 4G ETD, Bruker Daltonics).

Figure 3 Roepstorff-Fohlmann-Biemann nomenclature for fragment ions. The generic chemical structure of a peptide backbone is shown with the designation of fragment ions. The a-, b- and c- fragment ions extend from the N-terminus of the peptide and are numbered accordingly. Similarly, the x-, y- and z- fragment ions extend from the C-terminus of the peptide. The a- and $\mathrm{x}$ - fragment ions result from alkyl carbonyl bond cleavage, $\mathrm{b}$ - and $\mathrm{y}$ - ions result from peptide amide bond cleavage, c- and z- ions result from amino alkyl bond cleavage. Adapted from Steen and Mann (2004). 
Figure 4 Complementary structural information obtained by CID and ETD fragmentation of glycopeptides. (A) Deconvoluted CID MS/MS spectrum of the glycosylated tryptic peptide CGLVPVLAENYNK from human transferrin by ultra-high resolution quadrupole time-offlight tandem mass spectrometry (maXis 4G ETD, Bruker Daltonics). Extensive structural information for the glycan moiety of the glycopeptide obtained by CID fragmentation was used to identify the corresponding glycan structure by the GlycoQuest software programme (Bruker Daltonics). GlycoQuest correctly identified the glycan moiety as $\mathrm{Hex}_{5} \mathrm{HexNAc}_{4} \mathrm{NeuAc}_{2}$ in a glycan database search via its annotated fragment ions in the spectrum. (B) Deconvoluted ETD MS/MS spectrum of the same glycopeptide from human transferrin acquired by high-resolution ion trap tandem mass spectrometry (amaZon speed ETD, Bruker Daltonics). Following elucidation of the glycan moiety, an ion trap ETD MS/MS experiment was performed on the same glycopeptide and the acquired ETD spectrum was used in a MASCOT database search

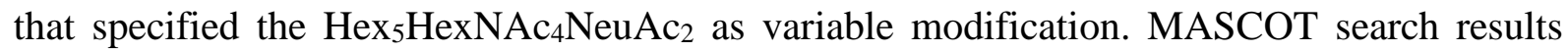
were imported into ProteinScape 3.1 (Bruker Daltonics) for visualisation. MASCOT correctly identified the peptide moiety as CGLVPVLAENYNK via its annotated c- and z- fragment ions (MASCOT score: 125). In addition, the glycosylation site of the peptide was readily identified from the deconvoluted spectrum as the glycan was preserved during ETD fragmentation of the peptide backbone. Symbols for the sugars are the following: hexose, circle; $\mathrm{N}$-acetylneuramic acid, diamond; N-acetylhexosamine, square.

Figure 5 Schematic representation of the typical steps in shotgun proteomics. Proteins are cut into proteolytic peptides by peptidases or chemicals (e.g. trypsin). The resulting peptide mixture is desalted and concentrated by solid phase extraction prior to liquid chromatography with online tandem mass spectrometry analysis. Peptides are separated by reversed phase chromatography and eluting peptides are subjected to mass spectrometric analysis. In time, the 
mass spectrometer will first acquire a survey scan $\left(\mathrm{MS}^{1}\right.$ level) to measure the precursor ion mass to charge ratio which is used to calculate the peptide mass. Next, the mass spectrometer isolates a precursor ion, which is fragmented and its fragment ions are analysed in a second round of mass spectrometry (MS/MS or $\mathrm{MS}^{2}$ level). Once a set number of $\mathrm{MS}^{2}$ spectra is recorded, the cycle of $\mathrm{MS}^{1}$ and related $\mathrm{MS}^{2}$ spectra acquisition is repeated till the end of the analysis to collect tandem mass spectrometry data for as many peptides as possible. After the LC-MS/MS analysis, vendor-specific software is used to extract data for the precursor- and fragment ions, which is used in subsequent protein sequence database searches. For each acquired fragmentation spectrum, the database search algorithm will attempt to match the experimental data with in-silico data for all available peptides in the sequence database. Ideally, each MS/MS spectrum provides a unique peptide identification, which is used to infer protein identifications in the final stage of MS/MS database searches. Adapted from Wessels (2015).

Figure 6 Manual de novo sequencing result for a CID MS/MS spectrum of the tryptic BSA peptide $\mathrm{YIC}_{(\mathrm{CAM})}$ DNQDTISSK acquired by ultra-high resolution quadrupole time of flight tandem mass spectrometry (maXis 4G ETD, Bruker Daltonics). The mass difference between successive fragment ion peaks was used to determine part of the sequence for this peptide in Data Analysis 4.2 software (Bruker Daltonics). Each amino acid that could be determined using subsequent fragment ions is annotated in the Figure with its according mass measurement error in u. Only dominant y- fragment ions were annotated for simplicity.

Figure 7 Isotopic distribution of horse cytochrome $c$ in a deconvoluted ESI-MS spectrum acquired by ultra-high resolution quadrupole time of flight tandem mass spectrometry (maXis 4G ETD, Bruker Daltonics). The monoisotopic peak is indicated in the spectrum. 
Figure 8 Native ESI-MS and deconvoluted MS (insert) spectra of the intact monoclonal antibody Trastuzumab by ultra-high resolution quadrupole time of flight mass spectrometry (maXis 4G ETD, Bruker Daltonics). The ESI-MS spectrum shows a wide charge state distribution for Trastuzumab ( $z=41$ up to $z=66$ ), which is typical for large proteins or complexes. The insert presents the deconvoluted spectrum of Trastuzuman from which the different configurations of heavy chains in the monoclonal antibody complex can be detected. The mass differences between subsequent peaks correspond with the mass of either a fucose (Fuc) or hexose (Hex) as annotated in the Figure. Glycan nomenclature to label spectral peaks is as follows. G0: GlcNAc $2 \mathrm{Man}_{3} \mathrm{GlcNAc}_{2}$, G0F: GlcNAc $\mathrm{Man}_{3} \mathrm{GlcNAc}_{2} \mathrm{Fuc}_{1}, \mathrm{G} 1 \mathrm{~F}$ : GalGlcNAc $_{2} \mathrm{Man}_{3} \mathrm{GlcNAc}_{2} \mathrm{Fuc}_{1}$, G2F: Gal ${ }_{2} \mathrm{GlcNAc}_{2} \mathrm{Man}_{3} \mathrm{GlcNAc}_{2} \mathrm{Fuc}_{1}$.

Figure 9 Two-dimensional isoelectric focussing SDS-PAGE reference map of the soluble cell fraction of Methanothermobacter thermautotrophicus. (A) Soluble proteins were separated in the first dimension (horizontal) by IEF ( $\mathrm{pH} 4-7$ ), followed by SDS PAGE in the second dimension (vertical). Proteins were visualised by silver staining. A number of "trains" representing proteoforms of a same protein are encircled. (B) Detail of the reference map obtained from a 2D IEF (pH 4.5-5.5) SDS-PAGE gel. This section is framed in Fig. 9A. Encircled are MTH701, which is a combination of MTH701 and MTH702, annotated as the Nand C-terminal parts, respectively, of acetyl-CoA synthase 2, and MTH1107, annotated as the biotin-carrying subunit of pyruvate carboxylase (PycB). However, MALDI-TOF MS reveals that biotin is absent in this subunit. Please note the various proteoforms of MTH1164 and of MTH1129, representing the alpha subunits of methylcoenzyme M reductase type I and type II, respectively. Protein extract was prepared from cells grown under hydrogen-limited conditions. 
Figure 10 Schematic representation of the various types of membrane proteins. (A) Proteins that span the lipid bilayer with one TMH having opposite orientations of their $\mathrm{N}$ - and C-termini. (B) Multi-pass membrane protein (here, 4 TMHs). (C) Protein attached to the membrane by hydrophobic and/ or ionic interactions. (D) Protein anchored in the membrane by a lipid chain. Adapted from Helbig et al. (2010).

Figure 11 Blue Native gel electrophoresis of soluble and membrane fractions of Methanothermobacter thermautotrophicus. (A) Two-dimensional reference (2D-BNE) map of the soluble fraction. This fraction was separated in the first dimension (horizontal) by $5-10 \%$ gradient BNE, followed by 10\% SDS PAGE in the second dimension (vertical) and subsequent staining with colloidal Coomassie. The same chromatographic conditions and staining method were applied in Figs. 11B-D. (B) Detail of a 2D-BNE gel of the membrane fraction solubilised with $\beta$-laurylmaltoside, showing monomers, dimers and higher-order supercomplexes of the archaeal $\mathrm{A}_{0} \mathrm{~A}_{1}$-type ATP synthase, and of the sodium-pumping $\mathrm{N}^{5}$-methyltetrahydromethanopterin:coenzyme M methyltransferase (MTR) complex. (C) Detail of a 2D-BNE gel of the membrane fraction solubilised with $\beta$-laurylmaltoside followed by crosslinking with "zerolength" 1-ethyl-3-(3-dimethylaminopropyl) carbodiimide (EDAC) in the presence of Nhydroxysulphosuccinimide (NHSS). The Figure shows monomeric and dimeric forms of the $\mathrm{A}_{0} \mathrm{~A}_{1}$-type ATP synthase (MTH957, MTH960), the MTR complex (MTH1157-1163) as well as a putative supercomplex of the ATP synthase and MTR. (D) Culture condition-dependent association between the heterodisulphide reductase (HDR) subunits A (MTH1878), B (MTH1879), C (MTH1381) and of the viologen-reducing hydrogenase (MVH) (not specified). HDR is found as two species associated with MVH, sizing approximately $400 \mathrm{kDa}$ and 300 kDa (arrows) when cells were grown under hydrogen limitation (MCR I condition). These 
species were hardly seen when growth had occurred under hydrogen excess (MCR II condition). Adapted from Farhoud et al. (2005).

Figure 12 Complexome profiling of membrane preparations of the anammox bacterium Kuenenia stuttgartiensis. Membrane preparations were subjected to 4-16\% (top, left) and 312\% gradient (top, right) BNE. After electrophoresis and gel staining with colloidal Coomassie, gels were each cut in about 65 equally sized pieces and each of these pieces was tryptically digested and analysed by LC-MS/MS, yielding in total 1400 unique proteins. Each of these proteins was label-free quantified for each gel piece. The large central part of the Figure shows the gel migration profile (horizontal direction) of each of these $\sim 1400$ proteins (vertical direction) displayed as heat maps. Next, migration profiles were hierarchically clustered (righthand side of the central part of the Figure) as to reveal proteins that co-migrated in the gels. In the bottom part a detail of the central Figure is displayed, establishing the co-migration of all subunits of NADH:quinone oxidoreductase (complex I; kuste2660-72), except for the very hydrophobic subunits NuoK (kuste2669; 3 TMHs, MW 11.2 kDa) and NuoN (kuste1172; 12 TMHs, MW $52.9 \mathrm{kDa}$ ) that remained undetected. All observed subunits were found in a protein complex of about $620 \mathrm{kDa}$, which favourably compares the theoretical MW $575 \mathrm{kDa}$ of complex I.

Figure 13 Two-dimensional difference gel electrophoresis (DIGE) of Methanothermobacter thermautotrophicus cells grown under hydrogen-depleted and hydrogen excess conditions. The cytoplasmic proteins from the hydrogen-excess and hydrogen-limited cultured conditions were tagged with red and green fluorescent dyes, respectively. Equal amounts of protein from both cultures were mixed and tagged with a yellow fluorescent dye 
serving as an internal control. Prior to 2D IEF SDS-PAGE, same protein amounts of these three preparations were combined. Multiple spots (proteoforms) derived from translation initiation factor $2 \alpha($ IIF2 $\alpha$, MTH1872) are accentuated by the white frame. The online version of this paper will show the Figure in colour.

Figure 14 Schematic representation of top-down MS analysis of a hypothetical membranebound protein complex composed of three subunits localised in the periplasmic (grey), membrane (dark grey) and cytoplasmic (light grey) aspects of the bacterial cell, respectively. The membrane-inserted subunit (2 TMHs) is associated with lipid molecules (filled circles and sticks) and contains a PTM (black triangle). The cytoplasmic subunit harbours another PTM (grey circle). Upon volatilisation of the as-isolated native complex inside the mass spectrometer, the gradual increase of ion activation/ ionisation results in gas-phase dissociation of the complex, followed by fragmentation of the subunits. Hereafter, peptide fragments are sequenced and the PTMs are localised and identified.

Figure 15 Schematic representation of different MS-based methods to get structural information of a protein complex. The Figure uses the same hypothetical protein as in Fig. 14. (A) As-isolated protein. (B) After limited proteolysis. Peptide fragments can be sequenced and PTMs identified and localised. (C) After probing of specific amino acids with chemical reagents, with low-molecular-weight dyes (“protein painting”), with in situ-generated hydroxyl radicals, or after deuterium exchange of amino acids amide hydrogen atoms. (D) After chemical crosslinking of neighbouring amino acid residues within or between the different subunits. 
Figure 16 Oxidative stress-related phenomena and protein repair. (A) Generation of reactive oxygen species (ROS) by subsequent one-electron reductions of $\mathrm{O}_{2}$. The superoxide radical $\left(\mathrm{O}_{2}\right)$ is converted into hydrogen peroxide $\left(\mathrm{H}_{2} \mathrm{O}_{2}\right)$ and $\mathrm{O}_{2}$ by superoxide dismutase (SOD). Catalase converts $\mathrm{H}_{2} \mathrm{O}_{2}$ into water and $\mathrm{O}_{2}$, whereas NADPH-dependent alkyl hydroperoxide reductase (Ahp) or cytochrome $c$ peroxidase (not shown) reduces $\mathrm{H}_{2} \mathrm{O}_{2}$ to water. The hydroxyl radical $(\mathrm{OH})$ is produced from $\mathrm{H}_{2} \mathrm{O}_{2}$ by chemical reaction with $\mathrm{Fe}^{2+}$ (Fenton reaction). (B) Reaction of thiol groups in the protein with ROS, reactive nitrogen species (NO) or hypochlorite (HOCl) converting a thiol in sulphenic acid (-SOH), a nitrosyl derivative (SNO) or a sulphenylchloride (-SCl), respectively. The latter species are irreversibly oxidised to sulphinic $\left(-\mathrm{SO}_{2}{ }^{-}\right)$and sulphonic $\left(-\mathrm{SO}_{3}{ }^{-}\right)$acid. Alternatively, these species react with other thiols in the protein to form disulphides (lower branch) or with low-molecular-weight "redox buffers" (RSH; glutathione, bacillithiol, mycothiol) to form heterodisulphides (upper branch). Enzymatic reduction of these disulphides regenerates the original thiols, establishing protein repair.

Figure 17 Schematic representation of the organisation of mitochondrial OXPHOS complexes. The Figures schematically show complexes I, III, IV and V. Complex II (succinate dehydrogenase) that does not form part of supercomplexes (SC) is omitted for better clarity. (A) The "liquid state model" in which the different OXPHOS complexes act separately. Electron transfer between NADH-oxidising complex I and complex III is mediated by ubiquinone (Q), whereas cytochrome $c$ (c) transfers electrons from complex III to oxygenreducing complex IV. (B) SCs observed in mitochondria from different eukaryotic species. The Figure displays from left to right the SC of complexes I+III+IV catalysing NADH oxidation with oxygen reduction ("respirasome”), the SC of complexes I+III coupling NADH oxidation with cytochrome $c$ reduction, and the SC of complexes III+IV, which mediates ubiquinol oxidation and oxygen reduction. The number of individual complexes found in preparations of 
mitochondria from eukaryotic representatives is shown in parentheses below the roman numberings. (C) ATP synthase dimers residing on top of the cristae that are found in long strings perpendicular the plane of the Figure. The direction of proton $\left(\mathrm{H}^{+}\right)$movements is indicated by arrows without any specification of proton-electron stoichiometry $\left(\mathrm{H}^{+} / 2 \mathrm{e}^{-}\right)$. Abbreviations: $\mathrm{M}$. matrix; IM, inner membrane; IMS, intermembrane space. 


\section{TABLES}

Table 1 System specifications of state-of-the-art instruments for each type of mass analyser ${ }^{\mathrm{a}}$.

\begin{tabular}{lllll}
\hline Mass analyser & $\begin{array}{l}\text { Mass } \\
\text { accuracy }\end{array}$ & $\begin{array}{l}\text { Resolving power } \\
(\mathrm{k})^{\mathrm{b}}\end{array}$ & $\begin{array}{l}\text { Dynamic range } \\
(\mathrm{k})^{\mathrm{b}}\end{array}$ & Mass range $(\mathrm{m} / \mathrm{z})$ \\
\hline FT-ICR & $<1 \mathrm{ppm}$ & 260 & 10 & $100-10.000$ \\
Orbitrap & $<1 \mathrm{ppm}$ & 107 & 5 & $50-6000$ \\
Time-of-Flight & $<1 \mathrm{ppm}$ & 80 & 100 & $50-20.000^{\mathrm{c}}$ \\
& & & & $50-400.000^{\mathrm{d}}$ \\
Ion trap & $<0.35 \mathrm{Da}$ & 10 & 10 & $50-6000$ \\
Quadrupole & $<0.2 \mathrm{Da}$ & 2 & 10 & $50-2000$ \\
\hline
\end{tabular}

aSpecifications were obtained from: Bruker, www.bruker.com; Thermo Scientific,

http://www.thermoscientific.com/; Waters, www.waters.com; AB Sciex, www.sciex.com; Agilent

Technologies, www.agilent.com; Shimadzu, www.shimadzu.com; JEOL USA: www.jeolusa.com.

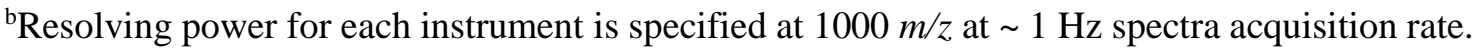

Resolving power is calculated as $m / \Delta m_{50 \%}$.

cESI orthogonal time-of-flight in reflectron mode.

${ }^{\mathrm{d}}$ MALDI time-of-flight mass analysers in linear mode. 
Table 2 Cytoplasmic membrane proteomes of selected prokaryotic species.

\begin{tabular}{|c|c|c|}
\hline Species & Method & Reference \\
\hline Bacteria, general & $2 \mathrm{DE}$ & Wilmes and Bond (2004) \\
\hline Bacillus subtilis & $2 \mathrm{DE}$ & Eymann et al. (2004) \\
\hline Campylobacter jejuni & $2 \mathrm{DE}+$ shotgun & Cordwell et al. (2008) \\
\hline Chlorobium tepidum & $2 \mathrm{DE}+$ shotgun & Kouyianou et al. (2010) \\
\hline Corynebacterium glutamicum & Shotgun & Schluesener et al. (2005 \\
\hline Enterococcus faecalis & BNE & Maddalo et al. (2011) \\
\hline \multirow[t]{3}{*}{ Escherichia coli } & $2 \mathrm{DE}$ & Lai et al. (2004) \\
\hline & $\mathrm{BNE}$ & Lasserre et al. (2006) \\
\hline & $\mathrm{BNE}$ & Stenberg et al. (2005) \\
\hline Francisella tularensis & $\mathrm{BNE}$ & Dresler et al. (2011) \\
\hline Helicobacter pylori & BNE & Pyndiah et al. (2007) \\
\hline Kuenenia stuttgartiensis & shotgun & Neumann et al. (2014) \\
\hline Methanothermobacter & $\mathrm{BNE}$ & Farhoud et al. (2005) \\
\hline \multicolumn{3}{|l|}{ thermautotrophicus } \\
\hline Mycobacterium bovis & $2 \mathrm{DE}+$ shotgun & Målen et al. (2008) \\
\hline Mycobacterium tuberculosis & Shotgun & Sinha et al. (2005 \\
\hline Paracoccus denitrificans & $\mathrm{BNE}$ & Stroh et al. (2004) \\
\hline Porphyromonas gingivalis & $\mathrm{BNE}$ & Glew et al. (2014) \\
\hline Staphylococcus aureus & $2 \mathrm{DE}+$ shotgun & Scherl et al., 2005 \\
\hline Streptococcus pneumoniae & Shotgun & Choi et al. (2010) \\
\hline
\end{tabular}


Table 3 Differential expression by selected prokaryotic species in response to change in energy metabolism.

\begin{tabular}{|c|c|c|c|}
\hline Species & Condition & Method; quantitation & Reference \\
\hline \multirow[t]{2}{*}{ Escherichia coli } & Ethanol stress & Shotgun; SILAC & Soufi et al. (2015) \\
\hline & Amino acid supply & DIGE & $\begin{array}{l}\text { Lopez-Campistrous et } \\
\text { al. (2005) }\end{array}$ \\
\hline \multirow{2}{*}{$\begin{array}{l}\text { Geobacter } \\
\text { metallireducens }\end{array}$} & Aromatic e-donors & Shotgun; label-free & Heintz et al. (2009) \\
\hline & Electron donor excess & Shotgun; label-free & Marozava et al. (2014) \\
\hline $\begin{array}{l}\text { Geobacter } \\
\text { sulfurreducens }\end{array}$ & U(VI) e-acceptor & Shotgun; label-free & Orellana et al., (2014) \\
\hline $\begin{array}{l}\text { Metallosphaera } \\
\text { cuprina }\end{array}$ & $\begin{array}{l}\text { S-oxidation + C- } \\
\text { metabolism }\end{array}$ & Shotgun; label-free & Jiang et al. (2014) \\
\hline Neisseria gonorrhoeae & Biofilm/ anaerobiosis & Shotgun; label-free & Phillips et al. (2012) \\
\hline $\begin{array}{l}\text { Pseudoalteromonas } \\
\text { tunicata }\end{array}$ & Planktonic/ biofilm & 2DE/BNE & Hoke et al. (2011) \\
\hline Psychroflexus torquis & Light & Shotgun; label-free & Feng et al. (2015) \\
\hline Purple Photobacteria & Light & CNE & Niederman (2013) \\
\hline \multirow[t]{2}{*}{ Ralstonia eutropha } & Chemolithoautotrophy & 2DE/ 1DE & $\begin{array}{l}\text { Kohlmann et al. } \\
\text { (2011) }\end{array}$ \\
\hline & Denitrification & 2DE/ 1DE & $\begin{array}{l}\text { Kohlmann et al. } \\
\text { (2014) }\end{array}$ \\
\hline $\begin{array}{l}\text { Shewanella } \\
\text { decolorationis }\end{array}$ & Azo/ Fe3 ${ }^{+}$e-acceptor & $2 \mathrm{DE}$ & Wang et al. (2010) \\
\hline Shewanella oneidensis & Aerobic/ microaerobic & Shotgun; label-free & Fang et al. (2006) \\
\hline Synechocystis sp. & $\mathrm{C} / \mathrm{N}$ assimilation & Shotgun; label-free & Wegener et al. (2010) \\
\hline \multirow{2}{*}{$\begin{array}{l}\text { Syntrophomonas } \\
\text { wolfei }\end{array}$} & Butyrate oxidation & $2 \mathrm{DE}$ & Schmidt et al. (2013) \\
\hline & Synthrophy & Shotgun; label-free & Sieber et al. (2015) \\
\hline
\end{tabular}


Table 4 Bacterial phosphoproteomes ${ }^{\mathrm{a}}$.

\begin{tabular}{|c|c|c|c|c|}
\hline $\begin{array}{l}\begin{array}{l}\text { Species } \\
\text { (proteins) }^{\mathrm{d}}\end{array} \\
\end{array}$ & Method & $\begin{array}{l}\text { P-proteins }{ }^{\mathrm{b}} \\
\text { (P-events) }\end{array}$ & $\mathrm{S}: \mathrm{T}: \mathrm{Y}(\%)^{\mathrm{c}}$ & Reference \\
\hline \multirow{3}{*}{$\begin{array}{l}\text { Bacillus subtilis } \\
\text { (4175) }\end{array}$} & $2 \mathrm{DE}$ & $28(9)$ & N.R. & Eymann et al. (2007) \\
\hline & Shotgun & 78 (103) & $69: 20.5: 10$ & Macek et al. (2007b) \\
\hline & Shotgun & 175 (339) & 74.8:17.7:7.1 & Lin et al. (2015) \\
\hline Campylobacter jejuni (1672) & $2 \mathrm{DE}$ & $36(58)$ & N.R. & Voisin et al. (2007) \\
\hline Escherichia coli & 2DE & $24(80)$ & N.R. & Soung et al. (2009) \\
\hline \multirow[t]{3}{*}{$(4140)$} & Shotgun & 79 (105) & $68: 23.5: 8.5$ & Macek et al. (2008) \\
\hline & Shotgun & $150(108)$ & 75.9:16.7:7.4 & Soares et al. (2013) \\
\hline & Shotgun & 393 (1088) & 69.5:21.8:7.7 & Lin et al. (2015) \\
\hline $\begin{array}{l}\text { Halobacterium salinarium } \\
\text { (2592) }\end{array}$ & Shotgun & $69(81)$ & $86: 12: 1$ & $\begin{array}{l}\text { Aivaloitis et al. } \\
\text { (2009) }\end{array}$ \\
\hline Helicobacter pylori (1447) & Shotgun & $76(126)$ & 42.8:38.7:18.5 & Ge et al. (2011) \\
\hline Klebsiella pneumoniae & Shotgun & $81(117)$ & N.R. & Lin et al. (2009) \\
\hline$(5501)$ & Shotgun & $559(663)$ & 72.9:13.7:12.9 & Lin et al. (2015) \\
\hline Lactobacillus lactis (1638) & Shotgun & $63(73)$ & $46.5: 50.5: 3$ & Soufi et al. (2008) \\
\hline $\begin{array}{l}\text { Mycobacterium tuberculosis } \\
\text { (3906) }\end{array}$ & Shotgun & $301(516)$ & 60:40:N.R. & Prisic et al. (2010) \\
\hline Mycoplasma pneumoniae & $2 \mathrm{DE}$ & $63(16)$ & N.R. & Schmidl et al. (2010) \\
\hline$(691)$ & Shotgun & $61(93)$ & $58: 37: 5$ & $\begin{array}{l}\text { van Noort et al. } \\
\text { (2012) }\end{array}$ \\
\hline $\begin{array}{l}\text { Pseudomonas aeruginosa } \\
\text { (5572) }\end{array}$ & Shotgun & $23(55)$ & $52.7: 32.7: 14.5$ & $\begin{array}{l}\text { Ravichandran et al. } \\
\text { (2009) }\end{array}$ \\
\hline $\begin{array}{l}\text { Pseudomonas putida } \\
\text { (5350) }\end{array}$ & Shotgun & $40(53)$ & 52.8:39.6:7.5 & $\begin{array}{l}\text { Ravichandran et al. } \\
\text { (2009) }\end{array}$ \\
\hline $\begin{array}{l}\text { Sinorhizobium meliloti } \\
\text { (6208) }\end{array}$ & Shotgun & $77(96)$ & 63:28:5 & Liu et al. (2015) \\
\hline $\begin{array}{l}\text { Staphylococcus aureus } \\
\text { (2767) }\end{array}$ & $2 \mathrm{DE}$ & 103 (68) & N.R. & Bäsell et al. (2014) \\
\hline $\begin{array}{l}\text { Streptococcus pneumoniae } \\
\text { (1814) }\end{array}$ & Shotgun & $102(163)$ & $47: 44: 9$ & Sun et al. (2010) \\
\hline $\begin{array}{l}\text { Streptomyces coelicolor } \\
(8152)\end{array}$ & Shotgun & $40(46)$ & $34: 52: 14$ & Parker et al. (2010) \\
\hline Synechocystis sp. PCC 6803 & 2DE & $32(8)$ & N.R. & Mikkat et al. (2014) \\
\hline$(3233)$ & Shotgun & $183(301)$ & & Spät et al. (2015) \\
\hline
\end{tabular}

${ }^{a}$ Adapted from Macek and Mijakovic (2011).

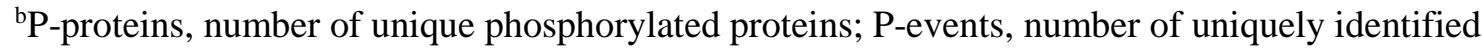
phosphorylation sites.

'S:P:Y, ratio (\%) between serine, threonine and tyrosine phosphorylation sites, respectively, in the phosphoproteome.

${ }^{\mathrm{d} N u m b e r}$ of protein-encoding genes as recorded in the NCBI genome database.

N.R., not reported. 
Table 5 Bacterial acetylomes

\begin{tabular}{lll}
\hline Species (proteins) $^{\mathrm{a}}$ & Ac-proteins (Ac-sites) $^{\mathrm{b}}$ & Reference \\
\hline Bacillus subtilis (4175) & $185(332)$ & Kim et al. $(2013)$ \\
Erwinia amylovora (3298) & $629(1355)$ & Kosono et al. $(2015)$ \\
Escherichia coli (4140) & $96(141)$ & Wu et al. $(2013)$ \\
& $85(125)$ & Yu et al. $(2008)$ \\
Geobacillus kaustophilus (3352) & $91(138)$ & Zhang et al. $(2009)$ \\
Mycobacterium tuberculosis (3908) & $114(253)$ & Lee et al. $(2013)$ \\
& $137(226)$ & Liu et al. $(2014)$ \\
Mycoplasma pneumoniae (691) & $658(1128)$ & Xie et al. $(2014)$ \\
Rhodopseudomonas palustris (4652) & $621(719)$ & van Noort et al. $(2012)$ \\
Salmonella enterica (4430) & $191(235)$ & Crosby et al. $(2012)$ \\
Staphylococcus aureus (2810) & $412(-)$ & Wang et al. $(2010)$ \\
Streptomyces roseosporus (6406) & $667(1143)$ & Zhang et al. $(2014)$ \\
Thermus thermophilus (2173) & $128(197)$ & Liao et al. $(2014)$ \\
Vibrio parahemolyticus (4449) & $656(1413)$ & Okanashi et al. $(2013)$ \\
\hline
\end{tabular}

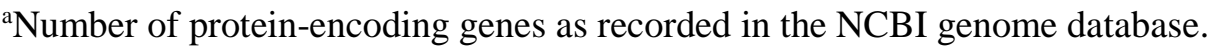

${ }^{\mathrm{b}}$ Ac-proteins, number of unique acetylated proteins; Ac-sites, number of unambiguously identified acetylation sites. 

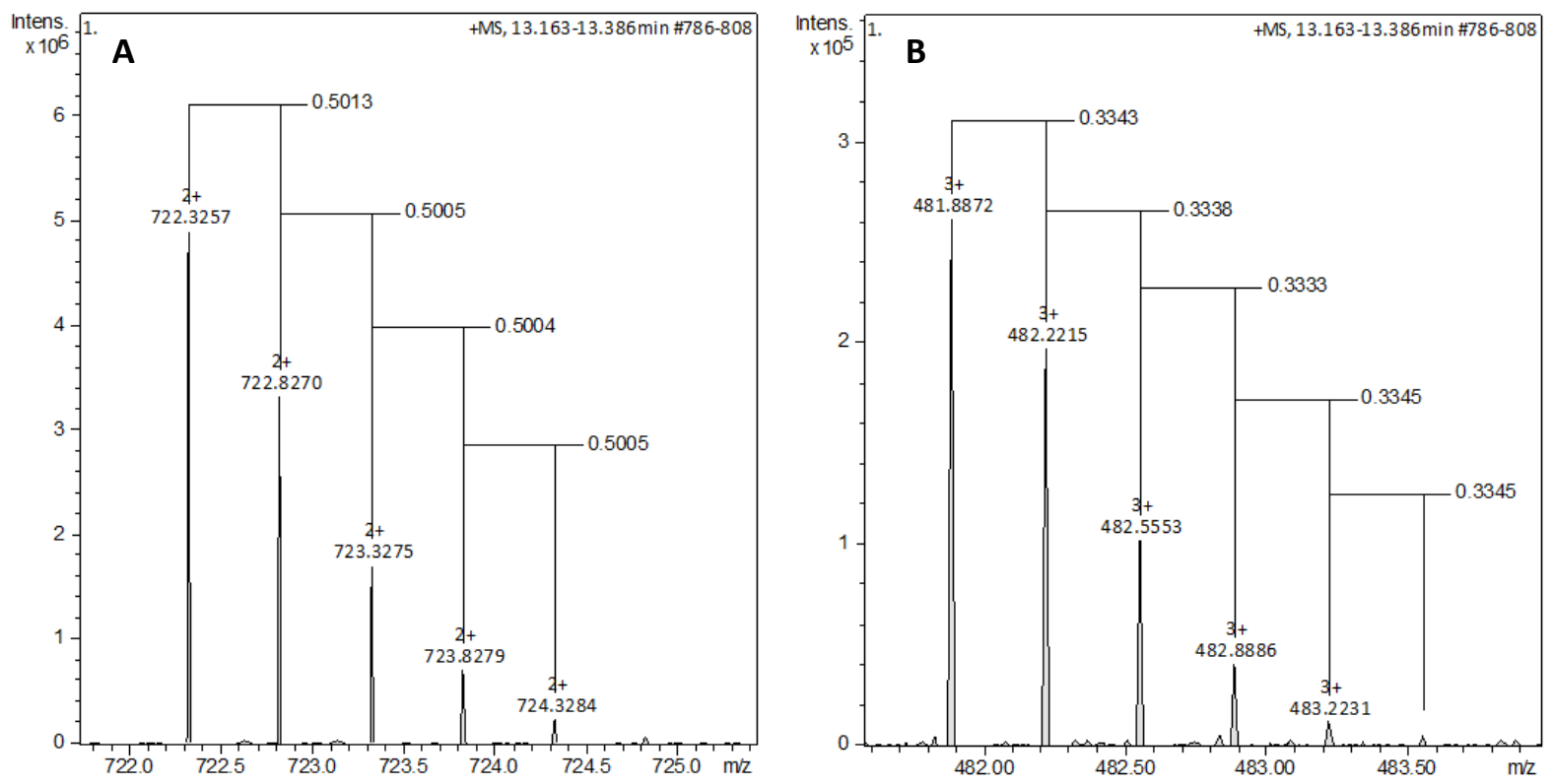

Figure 1 

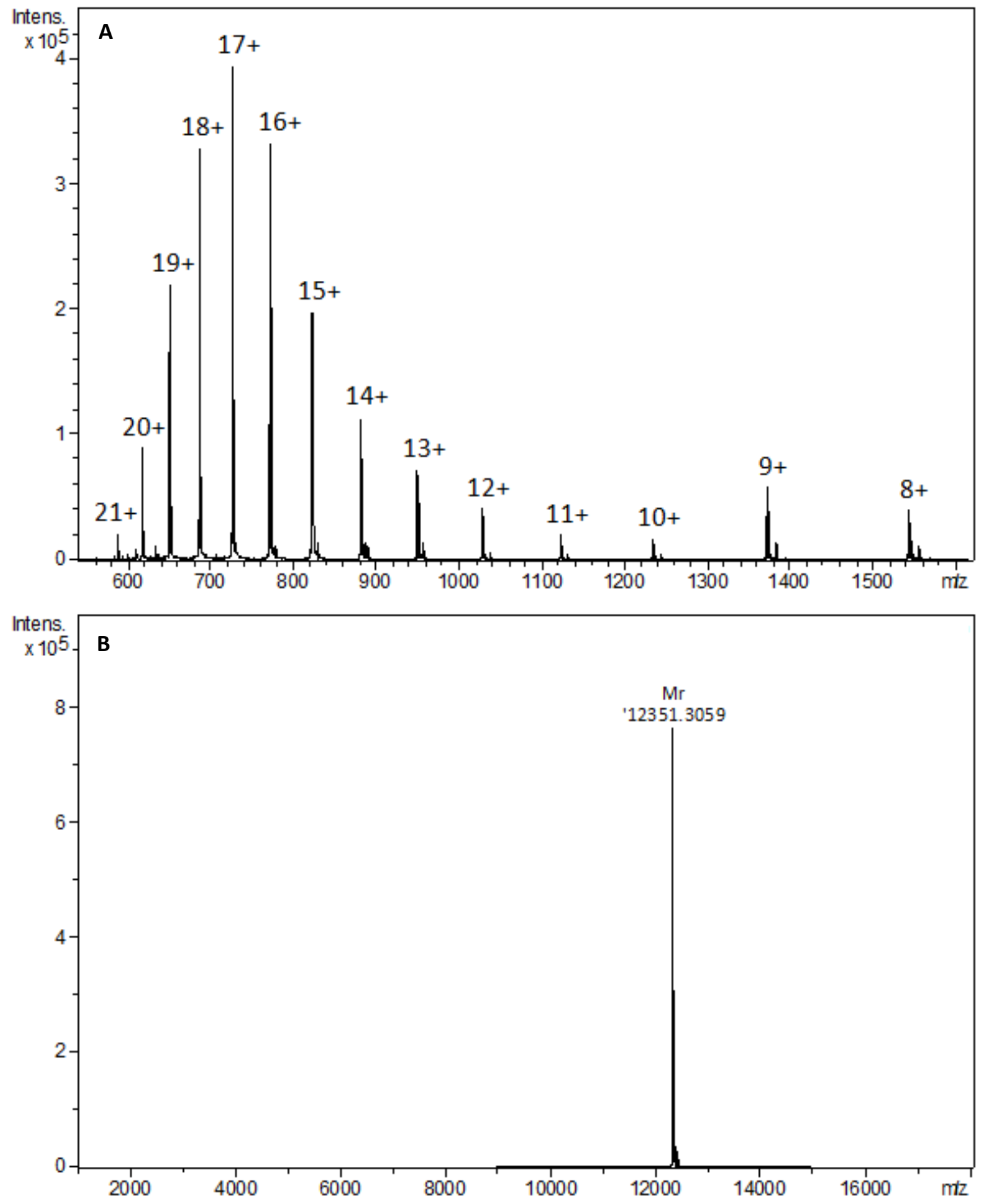

Figure 2 


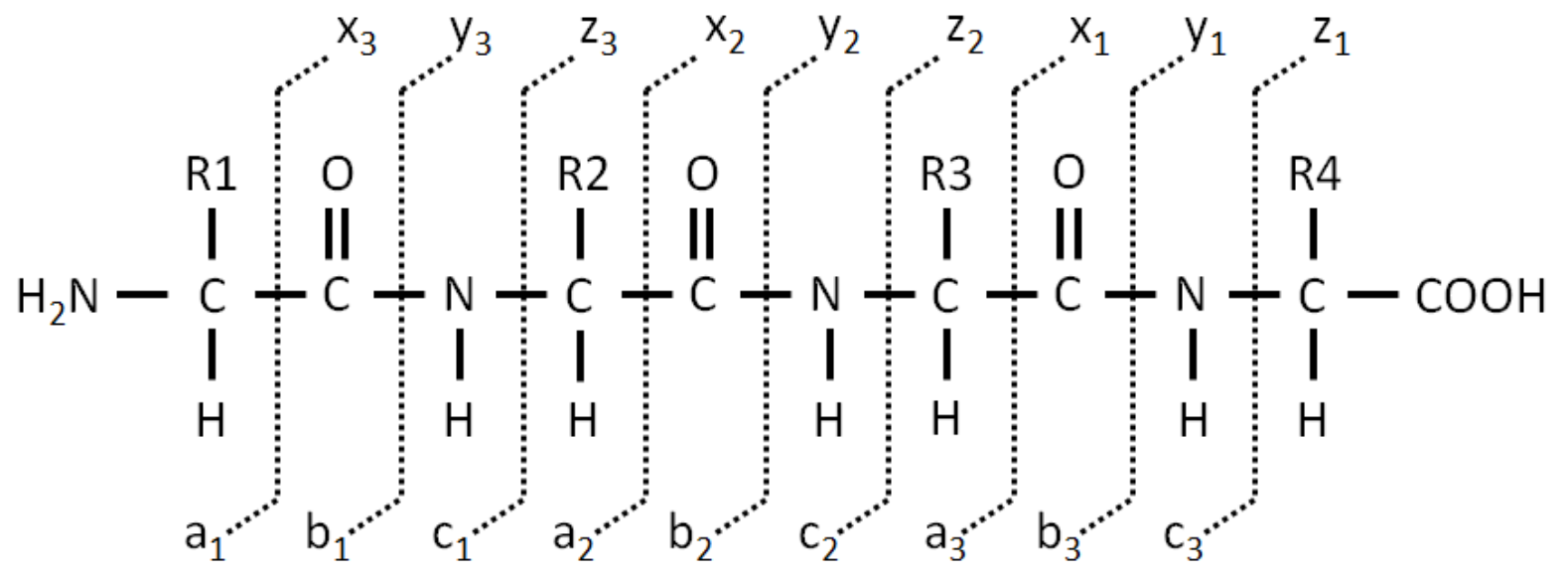

Figure 3

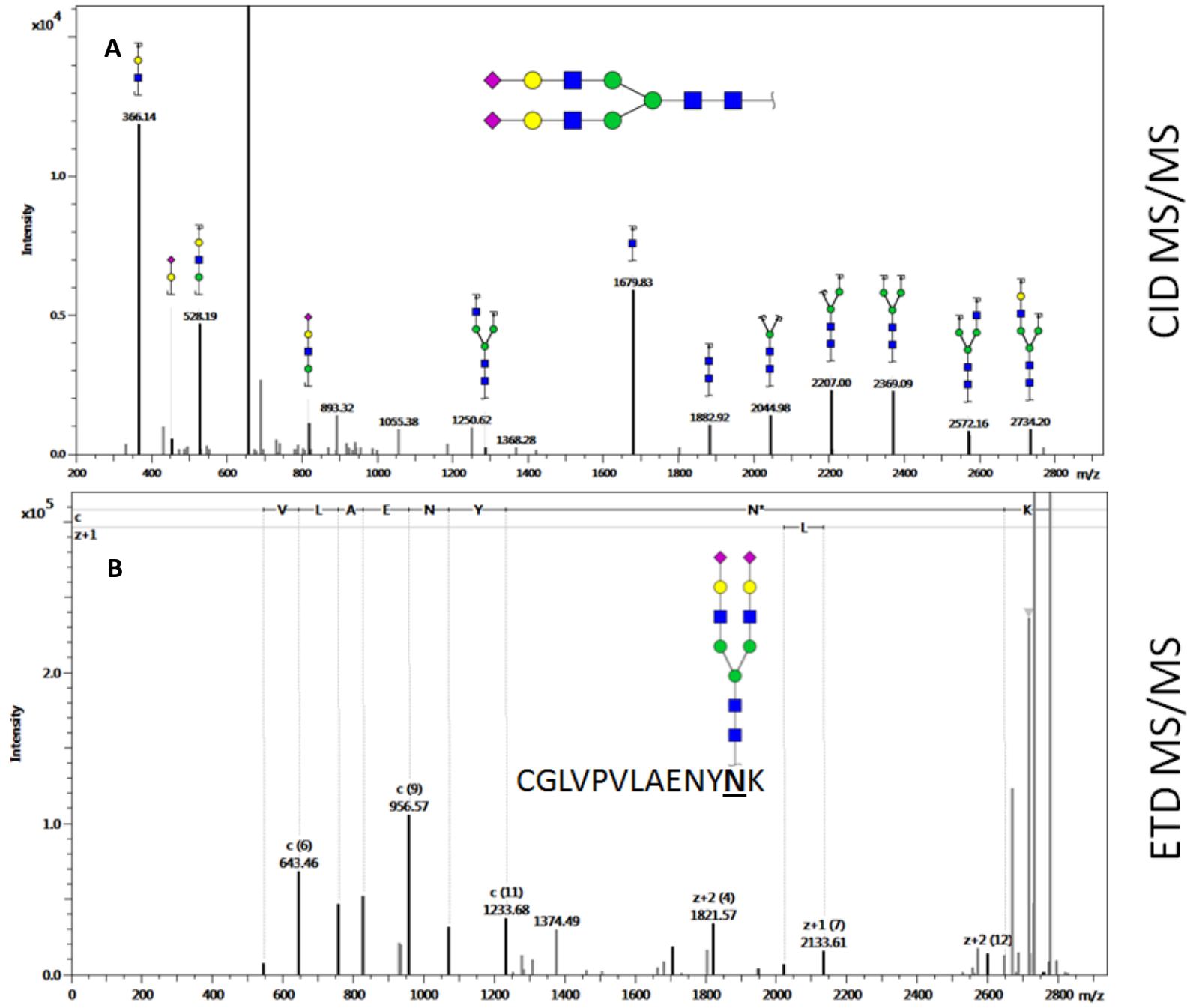

Figure 4 


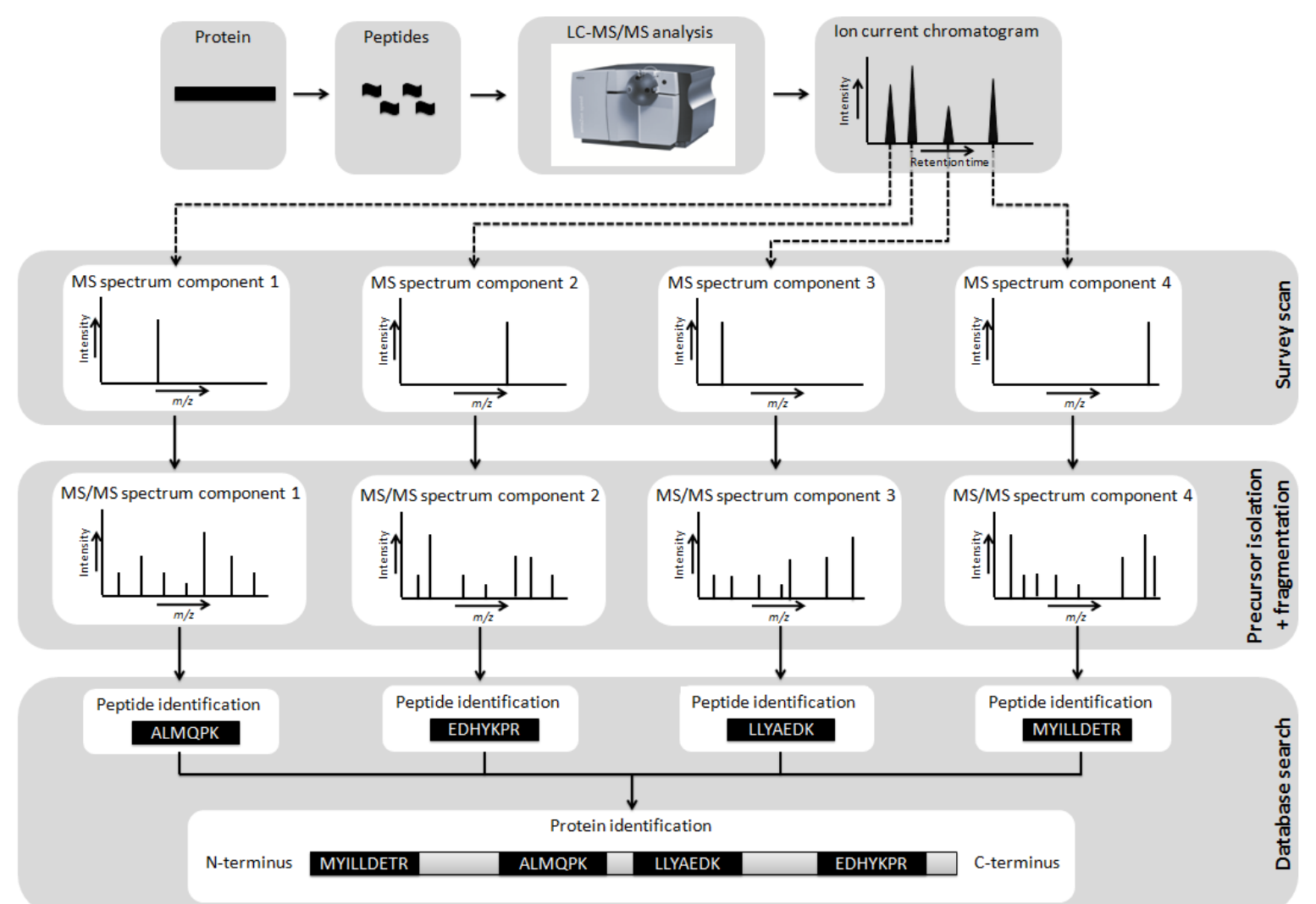

\section{Figure 5}

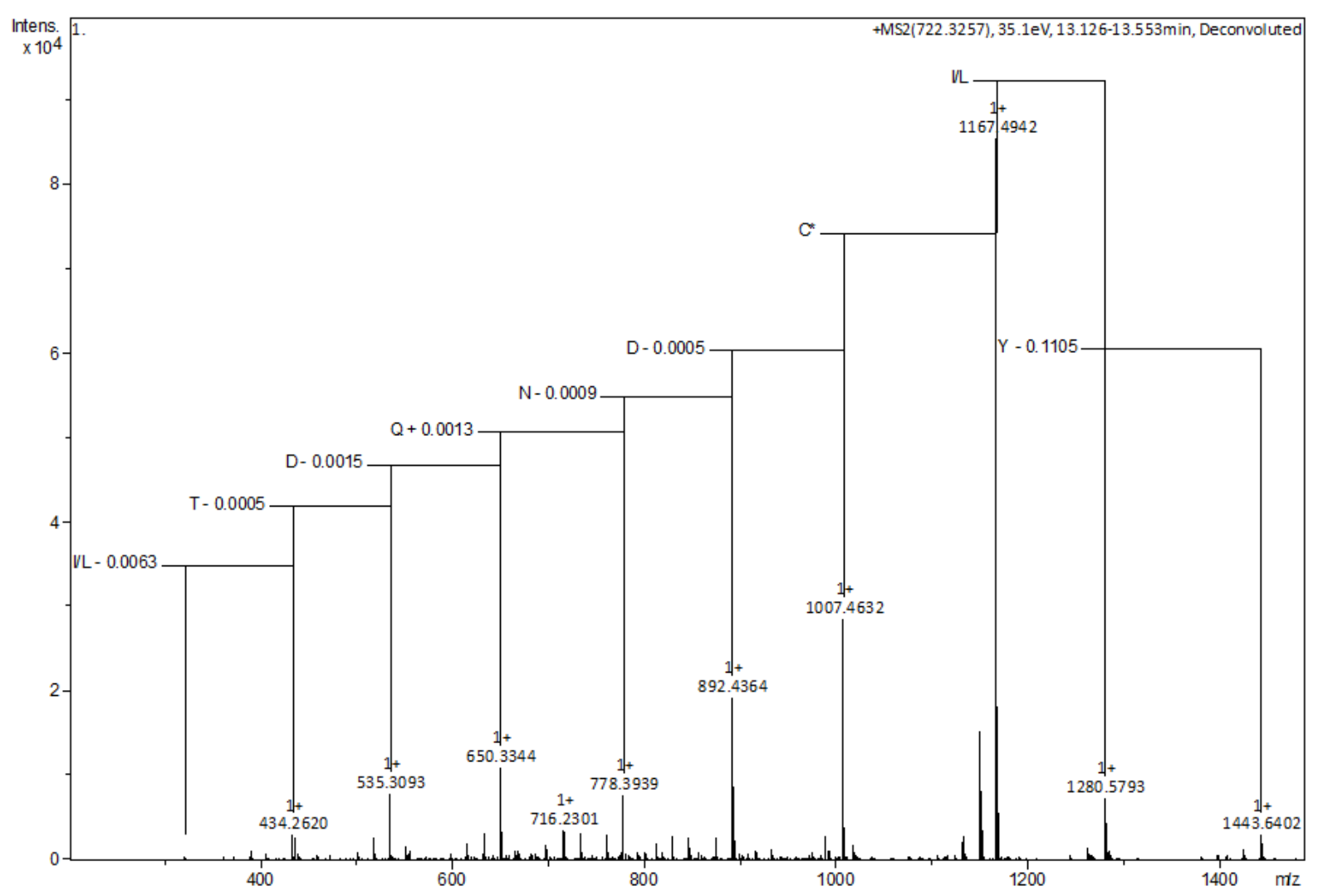

\section{Figure 6}




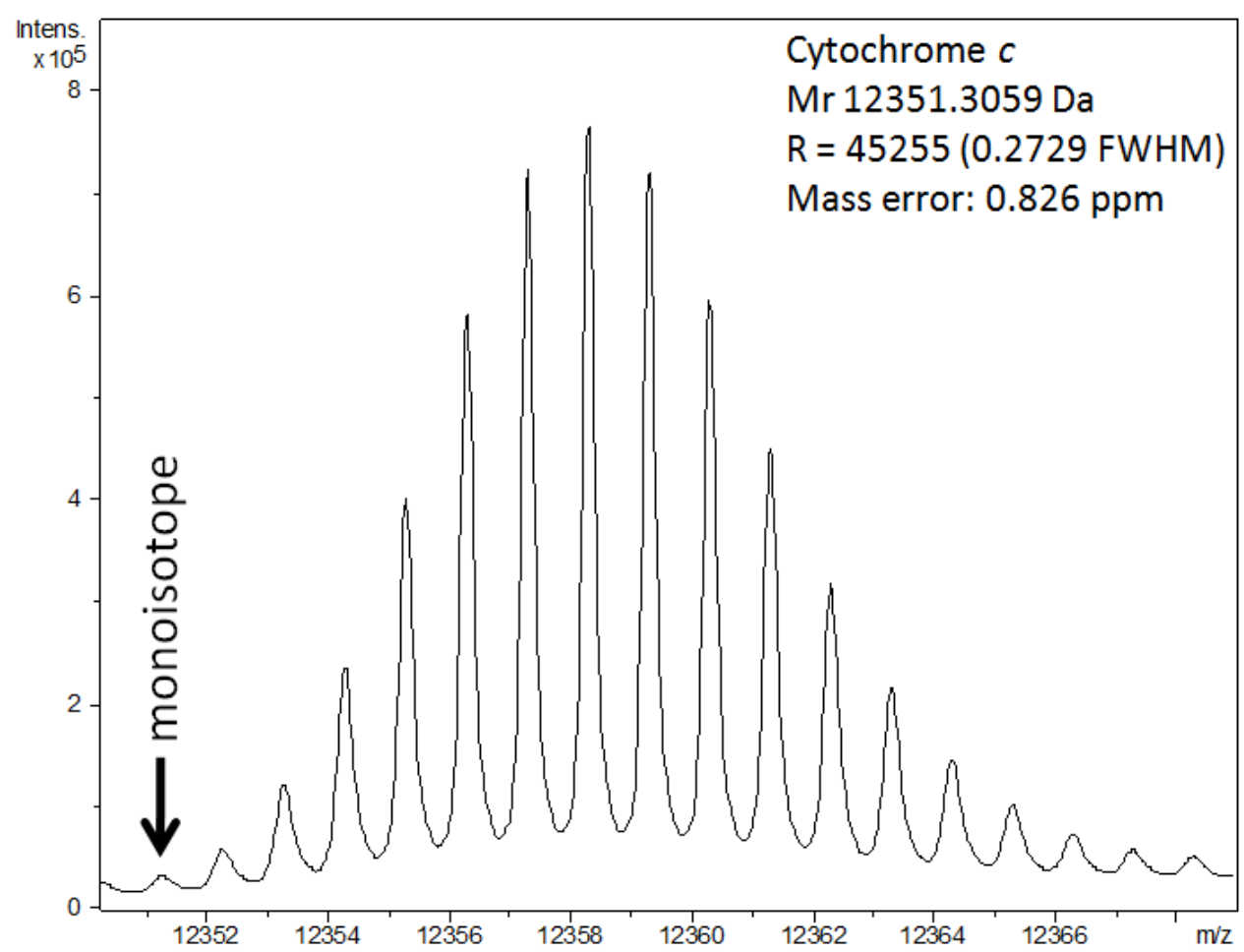

Figure 7

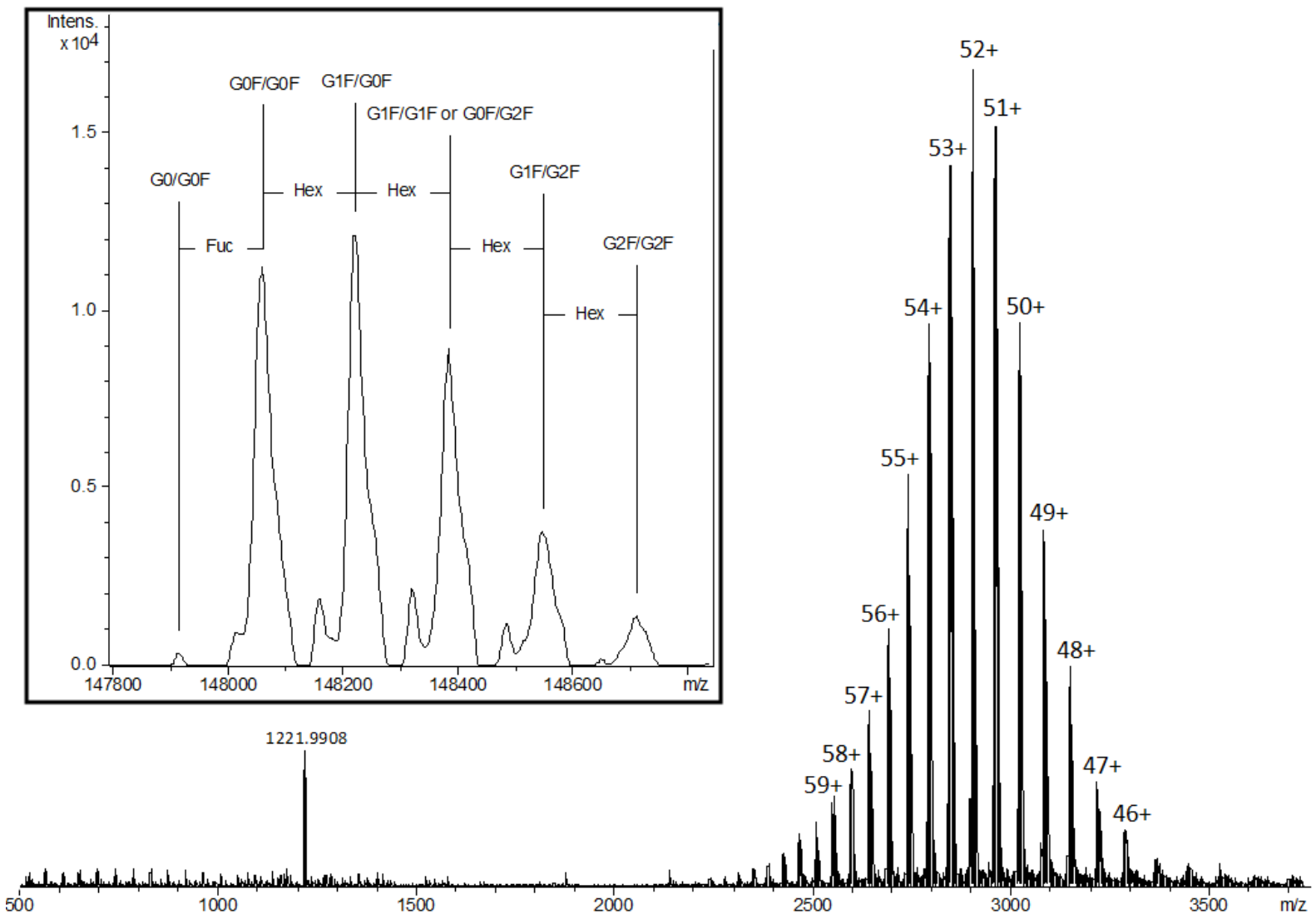

Figure 8 

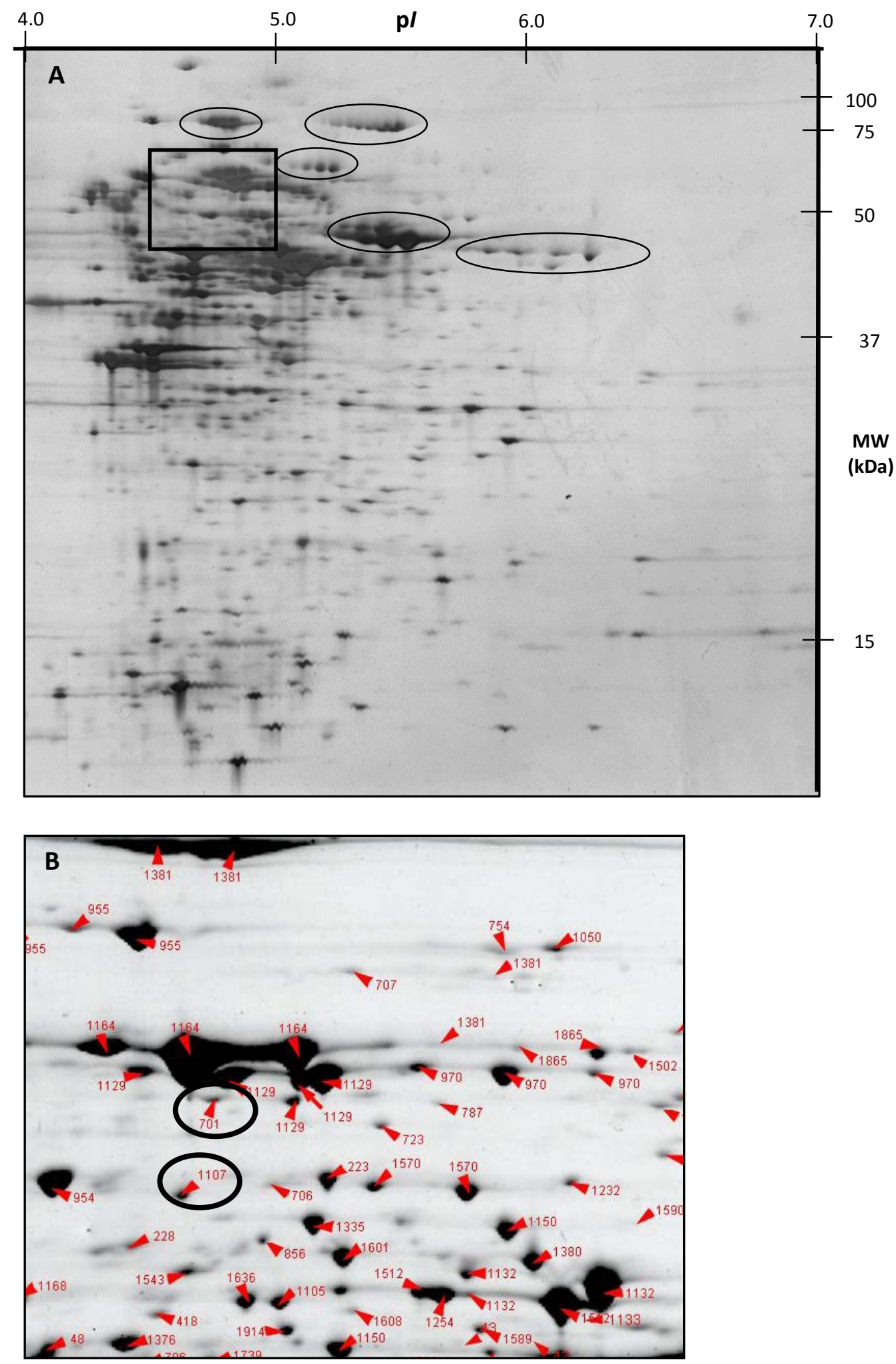

Fig. 9 


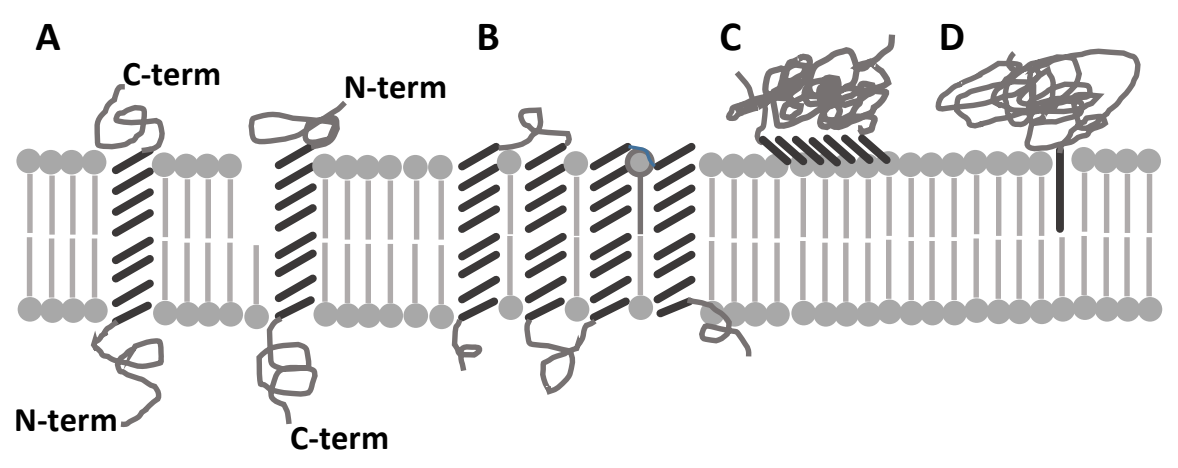

Fig. 10 

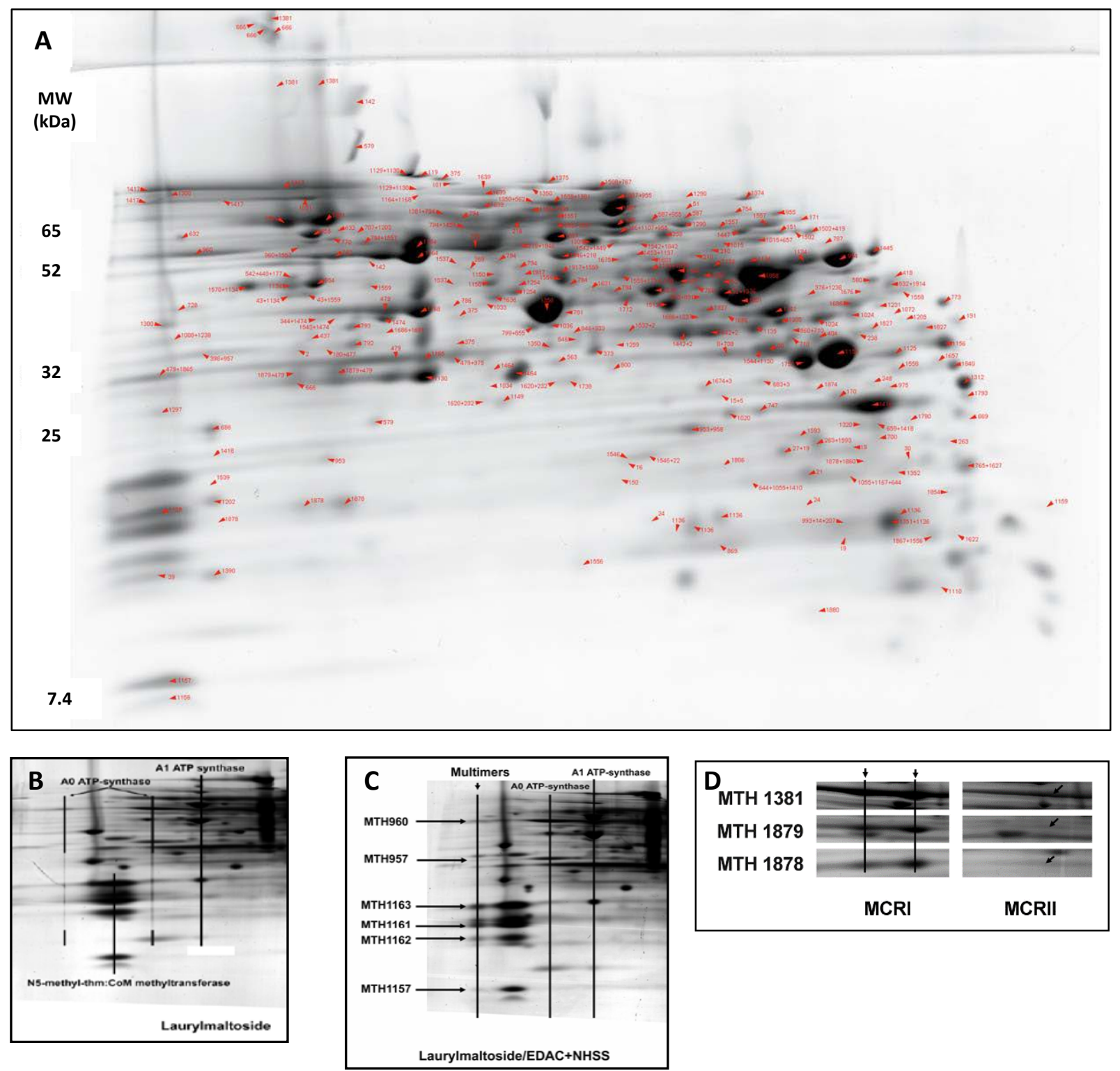

Fig. 11 

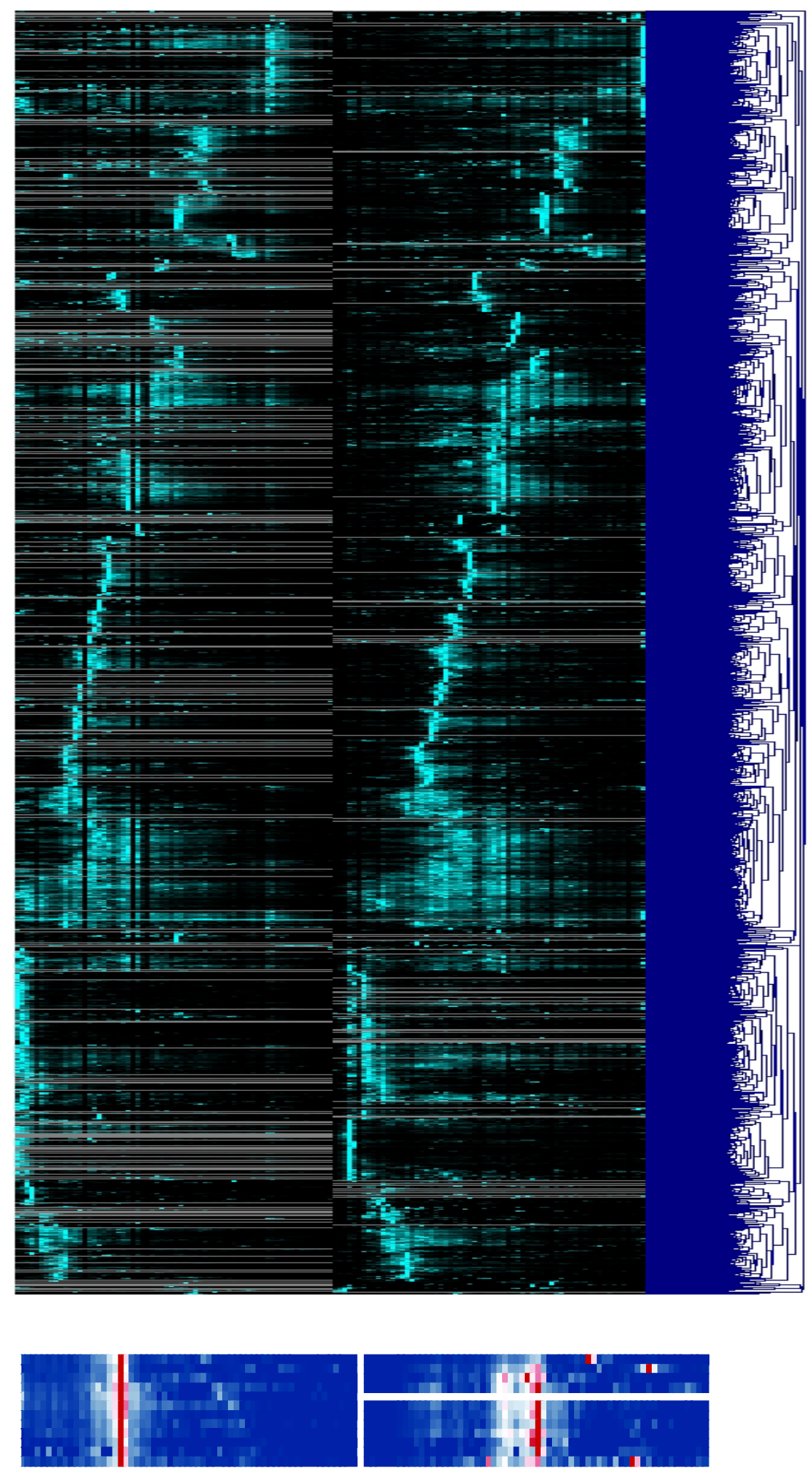

Fig. 12 


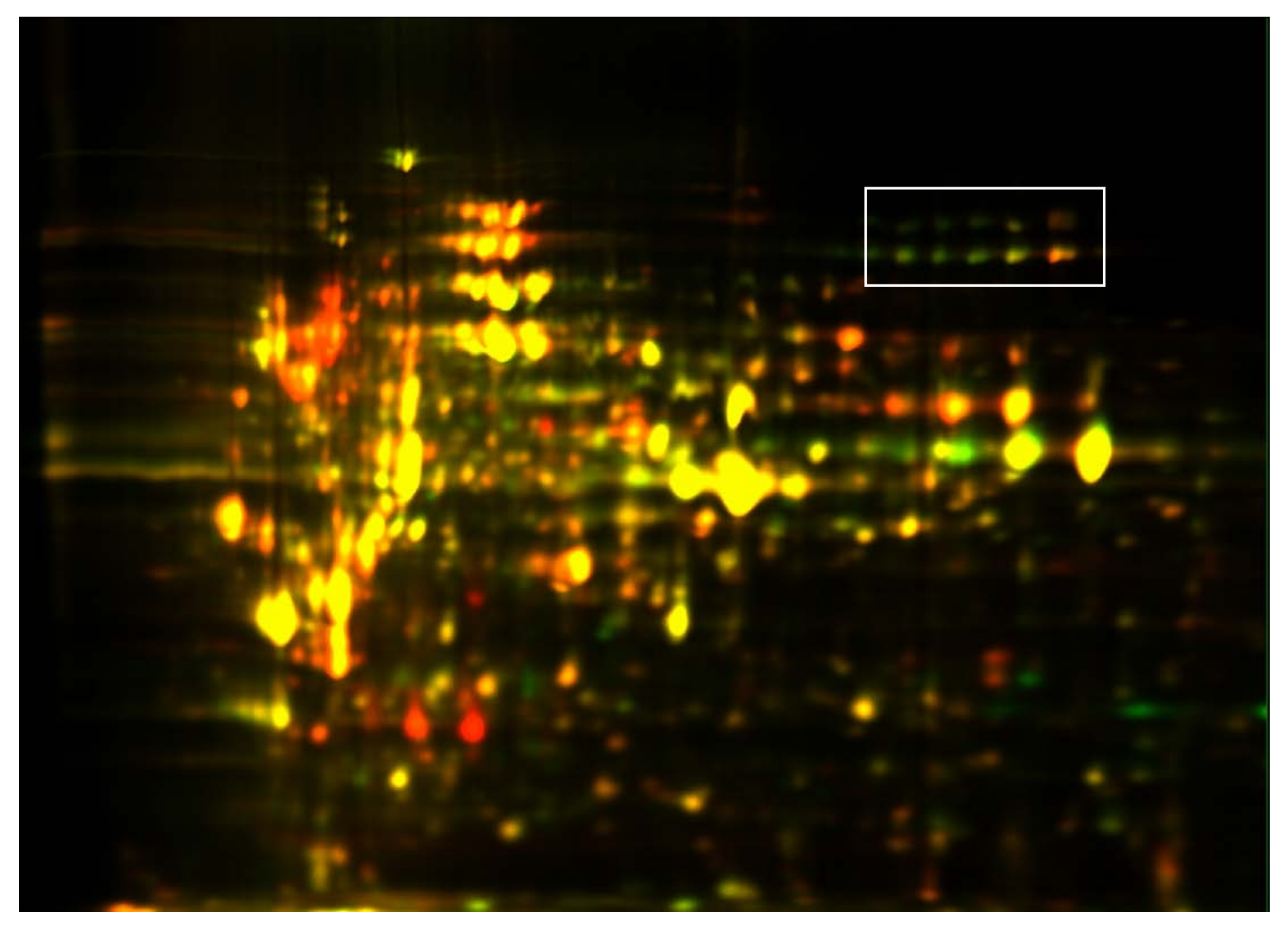

Fig. 13 
Intact

protein

complex

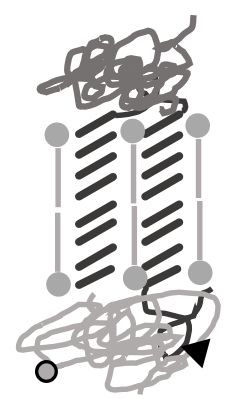

Ionisation

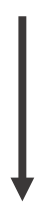

Gas-phase dissociation

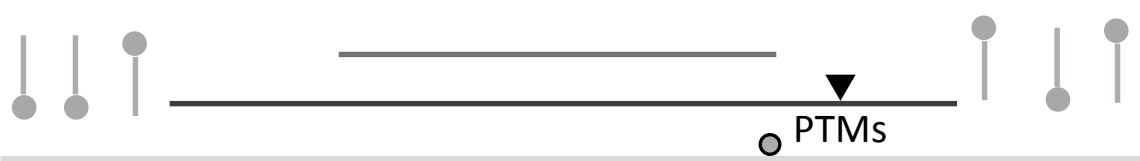

Peptide

fragmentation

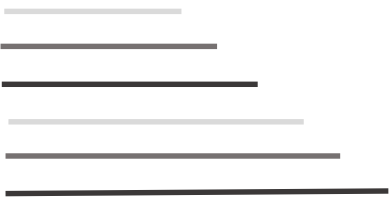

$\mathbf{N}$-terminus

C-terminus

Fig. 14
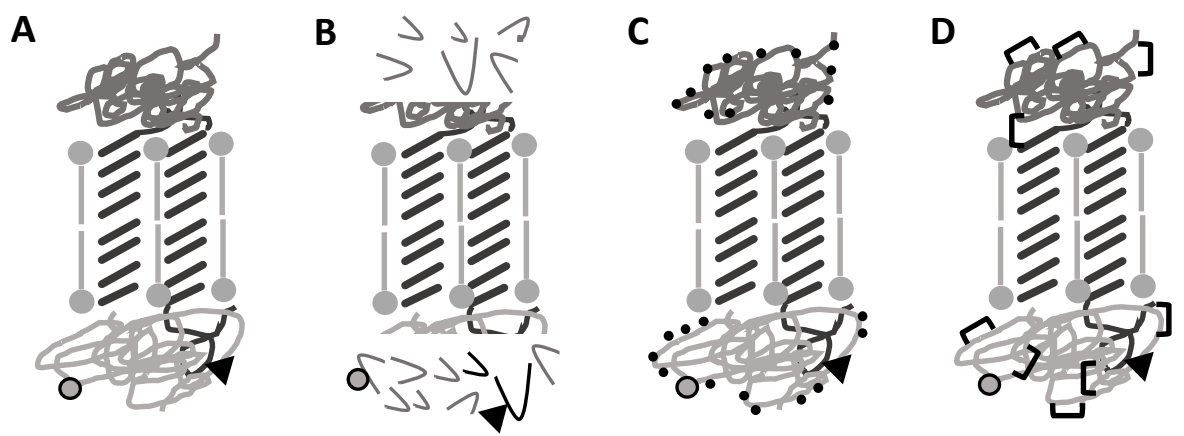

Fig. 15 

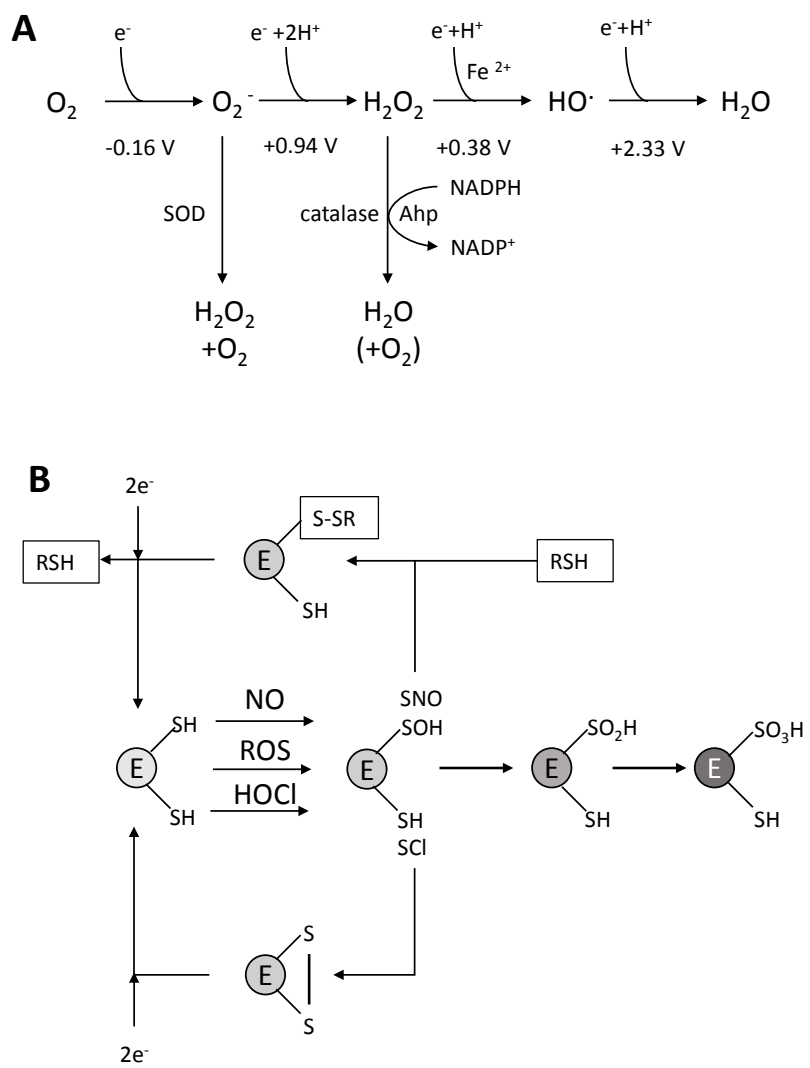

Fig. 16
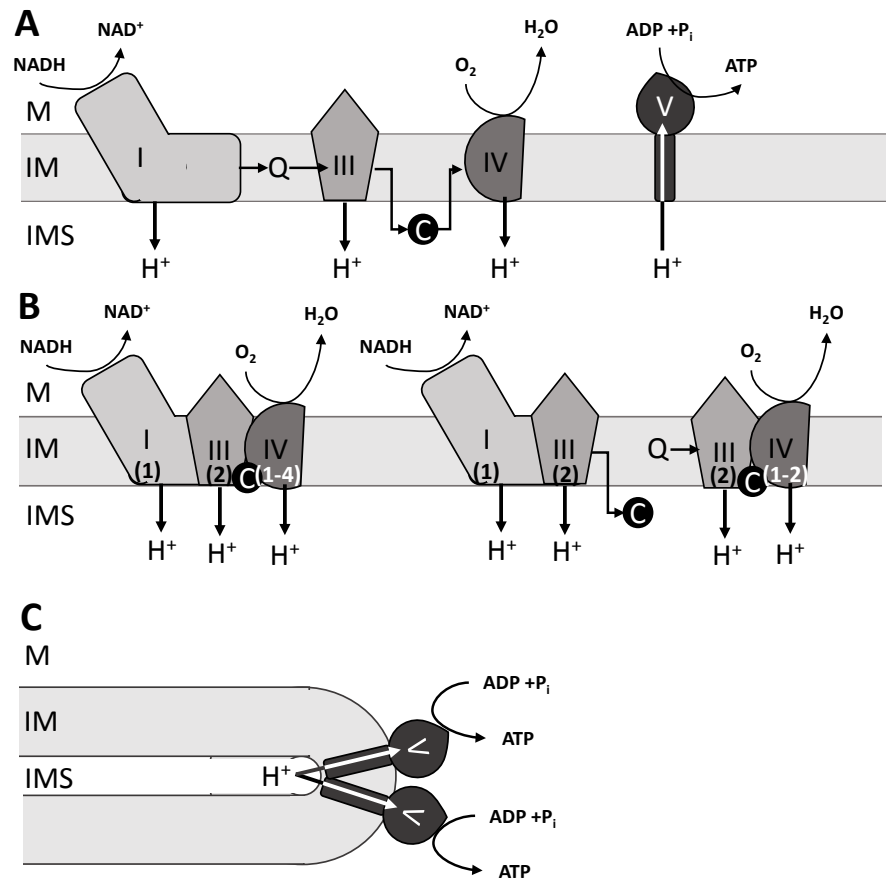

Fig. 17 